\title{
Robotic systems for breast biopsy using MRI and ultrasound imaging
}

Optimal guidance to target lesion in deformable tissue

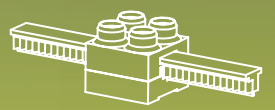

\section{Vincent Groenhuis}
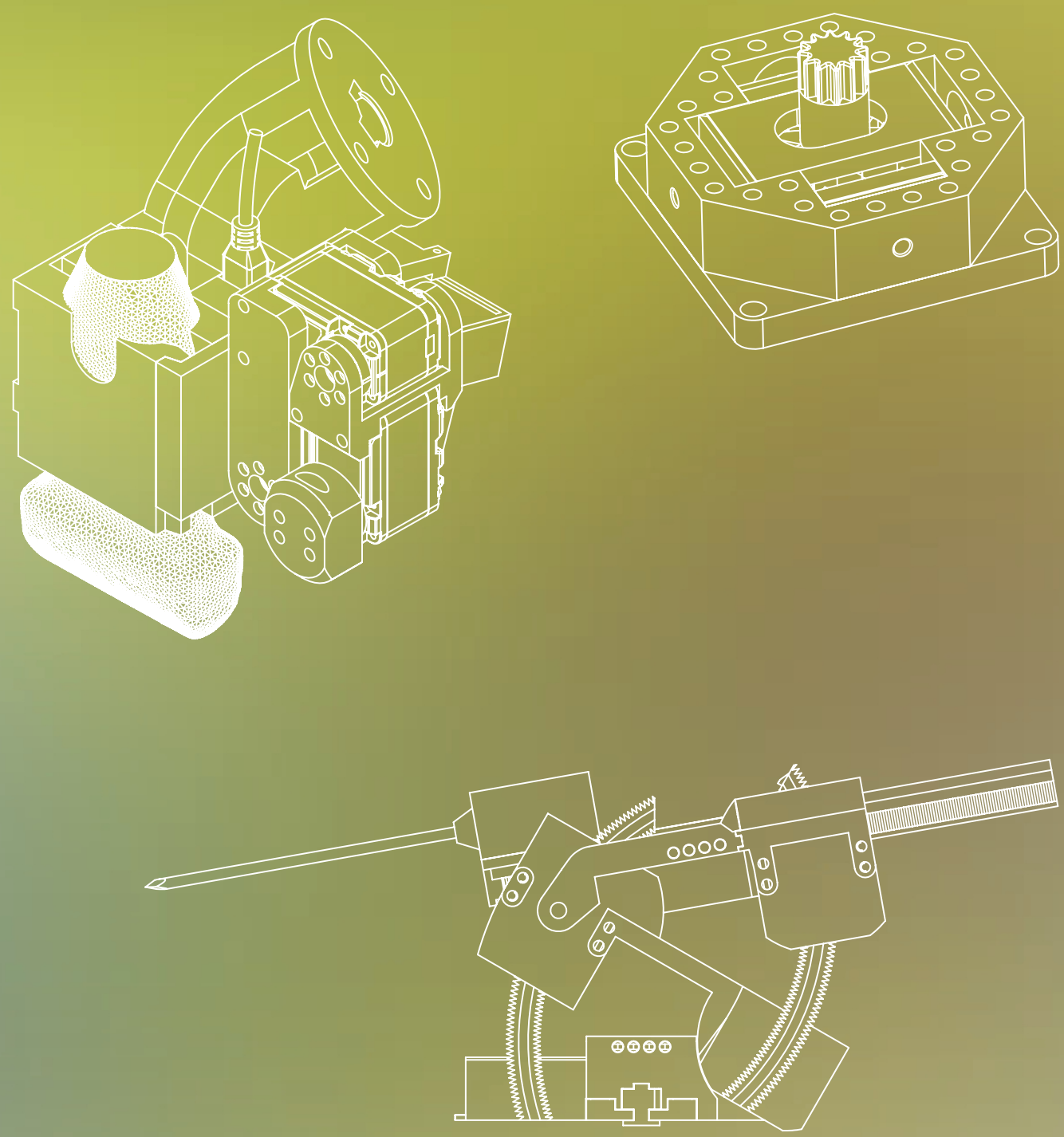



\section{ROBOTIC SYSTEMS FOR BREAST BIOPSY USING MRI AND ULTRASOUND IMAGING}

OPTIMAL GUIDANCE TO TARGET LESION IN

DEFORMABLE TISSUE

Vincent Groenhuis 
Graduation committee:

Chairman / secretary:

prof.dr. J.N. Kok University of Twente, NL

Promotor:

prof.dr.ir. S. Stramigioli University of Twente, NL

Co-promotor:

dr. F.J. Siepel University of Twente, NL

Committee members:

prof.

S. Perretta University of Strasbourg, France

prof.dr.ir.

P. Breedveld University of Delft, NL

prof.dr.ir.

S. Manohar

University of Twente, NL

prof.dr.ir.

C.L. de Korte

University of Twente, NL

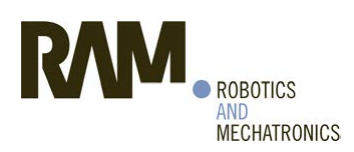

This research has been conducted at the group of Robotics and Mechatronics, Faculty of Electrical Engineering, Mathematics and Computer Science, University of Twente.

The MURAB consortium consists of the following seven partners: UT, RadboudUMC, ZGT, Verona, KUKA, Siemens and MUW.

The MURAB project has received funding from the EuFunded by the Horizon 2020
Framevorr prororamme of the ropean Union's Horizon 2020 research and innovation
European Union European Union $\quad$ programme under grant agreement No 688188.

\section{UNIVERSITY \\ OF TWENTE.}

Publisher:

University of Twente

Drienerlolaan 5

P.O. Box 217, 7500 AE, Enschede, The Netherlands

ISBN: $\quad$ 978-90-365-4892-2

DOI: $\quad 10.3990 / 1.9789036548922$

Printed by: Gildeprint.nl

Copyright@2020 Vincent Groenhuis, The Netherlands. 


\title{
ROBOTIC SYSTEMS FOR BREAST BIOPSY USING MRI AND ULTRASOUND IMAGING

\author{
OPTIMAL GUIDANCE TO TARGET LESION IN \\ DEFORMABLE TISSUE
}

\section{DISSERTATION}

\author{
to obtain \\ the degree of doctor at the University of Twente, \\ on the authority of the rector magnificus, \\ prof. dr. T.T.M. Palstra, \\ on account of the decision of the graduation committee, \\ to be publicly defended \\ on Thursday 9 January 2020 at 14:45
}

by

\section{Vincent Groenhuis}

born on November 15, 1983

in Nordhorn, Germany. 
This dissertation has been approved by:

Prof.dr.ir S. Stramigioli, Promotor

Dr. Françoise J. Siepel, Co-promotor

Copyright(C) 2020 Vincent Groenhuis, The Netherlands. All rights reserved. No parts of this thesis may be reproduced, stored in a retrieval system or transmitted in any form or by any means without permission of the author. Alle rechten voorbehouden. Niets uit deze uitgave mag worden vermenigvuldigd, in enige vorm of op enige wijze, zonder voorafgaande schriftelijke toestemming van de auteur. 


\section{SUMMARY}

In breast cancer screening the radiologist searches for suspicious lesions inside the breast. If the lesion is only visible on MRI then it is difficult to precisely target it for a biopsy. The current manual procedure is inaccurate and inefficient, so research has been done to develop suitable alternatives using robotics. Two different robotic system projects have been conducted to tackle the clinical challenge: the MURAB project and the Stormram project.

The MURAB project combines different imaging modalities including MRI, ultrasound, elastography and stereo vision to create a detailed patient-specific model for the biopsy. The setup consists of a metallic robot arm with endeffector positioned under a patient bed. The patient is first scanned in the MRI and then by the robotic arm, resulting in 3D scans in MRI, ultrasound and elastography. A patient-specific simulation model is created and the intervention planned, taking tissue deformations into account. The biopsy needle is manually inserted by the radiologist. The different sub-parts of the system were investigated in a broad range of phantom experiments, while preliminary experiments with the full setup on phantoms were conducted as well. The MURAB setup has shown that it is effectively able to apply deformation compensation techniques in targeting lesions.

The Stormram project takes a different approach into the same clinical challenge. An MR safe needle manipulator is placed inside the MRI scanner, allowing to insert the biopsy needle robotically straight after the MRI scan without moving the patient. The manipulator is actuated by pneumatic stepper motors which are entirely made of non-metallic materials and are extensively described and evaluated. A total of five distinct prototypes have been built within the Stormram project. The Stormram 4 has shown to have a needle positioning accuracy in MRI of $2 \mathrm{~mm}$.

Both the MURAB and Stormram projects show that it is possible to tackle the clinical challenge using a robotic system, taking tissue deformations into account. Several new technologies and combinations have been developed within both projects and these also demonstrate the value of the conducted research. 



\section{SAMENVATTing}

Bij borstkankeronderzoek zoekt de radioloog naar verdachte laesies in de borst. Sommige laesies zijn alleen zichtbaar op MRI en derhalve moeilijk om precies aan te prikken voor biopsie. De huidige handmatige procedure is onnauwkeurig en inefficiënt, dus er is onderzoek gedaan om geschikte alternatieven te ontwikkelen met behulp van robotica. Er zijn twee verschillende projecten uitgevoerd om de klinische uitdaging aan te gaan: het MURAB project en het Stormram project.

Het MURAB project combineert verschillende beeldvormingsmodaliteiten waaronder MRI, echografie (ultrasound), elastografie en beeldherkenning, om een gedetailleerd patiëntspecifiek model voor borstbiopsie te creëren. De opstelling bestaat uit een (metalen) robotarm met een manipulator geplaatst onder een patiëntbed. De patiënt wordt eerst gescand in de MRI en vervolgens door de robotarm, wat resulteert in 3D-scans in MRI, ultrasound en elastografie. Er wordt een patiëntspecifiek simulatiemodel gemaakt en de biopsie voorbereid, daarbij rekening houdend met voorspelde vervorming van het weefsel. De biopsienaald wordt met de hand ingebracht door de radioloog. De verschillende sub-onderdelen van het project werden onderzocht in fantoomexperimenten, terwijl experimenten met de volledige opstelling ook op fantomen werden uitgevoerd. Er is aangetoond dat de MURAB opstelling in staat is om effectief vervormingen te compenseren bij het aanprikken van laesies.

Het Stormram project gebruikt een andere aanpak voor de klinische uitdaging. Een MR-veilige naaldmanipulator wordt in de MRI-scanner geplaatst zodat de biopsienaald met de robot kan worden ingebracht na de MRI-scan zonder de patiënt te verplaatsen. De manipulator wordt bediend door pneumatische stappenmotoren welke volledig zijn gemaakt van kunststof materialen en deze zijn uitgebreid worden beschreven en geëvalueerd. Er zijn in totaal vijf verschillende prototypes gebouwd in de Stormram serie. Het vierde prototype, de Stormram 4, heeft een nauwkeurigheid van $2 \mathrm{~mm}$ bij het positioneren van de naald in de MRI.

Zowel het MURAB als het Stormram project tonen aan dat het mogelijk 
is om de klinische uitdaging aan te pakken met behulp van een robotsysteem, daarbij rekening houdend met weefsel-vervormingen. Verscheidende nieuwe technologieën en combinaties daarvan zijn ontwikkeld binnen beide projecten en deze hebben ook de waarde van het uitgevoerde onderzoek bewezen. 


\section{LIST OF PUBLICATIONS}

\section{Conference proceedings}

1. V. Groenhuis, F. J. Siepel, and S. Stramigioli. Miniaturization of MR Safe Pneumatic Rotational Stepper Motors. In IEEE/RSJ International Conference on Intelligent Robots and Systems, Macau, 2019. IEEE

2. M. E. M. K. Abdelaziz, D. Kundrat, M. Pupillo, G. Dagnino, T. M. Y. Kwok, W. Chi, V. Groenhuis, C. Riga, S. Stramigioli, and G.-Z. Yang. Toward a Versatile Robotic Platform for Fluoroscopy and MRI-Guided Endovascular Interventions : A Pre-Clinical Study. In IEEE/RSJ International Conference on Intelligent Robots and Systems, Macau, 2019. IEEE

3. A. V. Nikolaev, H. H. G. Hansen, L. de Jong, R. Mann, F. Siepel, A. Nikolaev, E. Tagliabue, B. Maris, V. Groenhuis, M. Caballo, I. Sechopoulos, and C. L. de Korte. Ultrasound-guided breast biopsy of ultrasound occult lesions using multimodality image co-registration and tissue displacement tracking. In International Congress on Ultrasonics 2019, page 45, Bruges, Belgium, 2019. doi: 10.1117/12.2513630

4. V. Groenhuis, F. Siepel, and S. Stramigioli. Dual-Speed MR Safe Pneumatic Stepper Motors. In Proceedings of Robotics: Science and Systems, Pittsburgh, Pennsylvania, 2018. doi: 10.15607/rss.2018.xiv.030

5. V. Groenhuis, F. J. Siepel, M. K. Welleweerd, J. Veltman, and S. Stramigioli. Sunram 5: An MR Safe Robotic System for Breast Biopsy. In Hamlyn Symposium on Medical Robotics, pages 82-83, 2018

6. V. Groenhuis, F. J. Siepel, J. Veltman, and S. Stramigioli. Design and characterization of Stormram 4: an MRI-compatible robotic system for breast biopsy. In IEEE/RSJ International Conference on Intelligent Robots and Systems (IROS) September 24-28, 2017, Vancouver, BC, 
Canada, volume 2017-Septe, pages 1746-1753, 2017. doi: 10.1109/IROS. 2017.8202256

7. M. E. Abdelaziz, V. Groenhuis, J. Veltman, F. Siepel, and S. Stramigioli. Controlling the Stormram 2: An MRI-compatible robotic system for breast biopsy. In Proceedings - IEEE International Conference on Robotics and Automation, pages 1746-1753, 2017. doi: 10.1109/ICRA. 2017.7989206

8. T. E. Chemaly, F. J. Siepel, S. Rihana, V. Groenhuis, F. Van der Heijden, and S. Stramigioli. MRI and Stereo Vision Surface Reconstruction and Fusion. In International Conference on Advances in Biomedical Engineering, ICABME, volume 2017-Octob, pages 1-4, 2017. doi: 10.1109/ICABME.2017.8167571

9. J. J. A. S. Uiterkamp, V. Groenhuis, F. J. Siepel, and S. Stramglioli. Design and Implementation of Autnomous Robotic Scanning of the Breast. In 6th Dutch Bio-Medical Engineering Conference 2017, volume 30, page 7522, Egmond, 2017

10. R. Spoor, M. Abayazid, F. Siepel, V. Groenhuis, and S. Stramigioli. Design and evaluation of a robotic needle steering manipulator for imageguided biopsy. In 6th Dutch Bio-Medical Engineering Conference 2017, Egmond aan Zee, 2017

11. V. Groenhuis, J. Veltman, and S. Stramigioli. Stormram 2: A MRICompatible Robotic System for Breast Biopsy. In Hamlyn Symposium on Medical Robotics, pages 52-53, 2016

12. V. Groenhuis, M. Chandrapal, S. Stramigioli, and X. Chen. Controlling pneumatic artificial muscles in exoskeletons with surface electromyography. 14th Mechatronics Forum International Conference, MECHATRONICS 2014, pages 451-457, 2014

\section{Journals}

13. F. Visentin, V. Groenhuis, B. Maris, D. Dall'Alba, F. Siepel, S. Stramigioli, and P. Fiorini. Iterative simulations to estimate the elastic properties from a series of MRI images followed by MRI-US validation. Medical and Biological Engineering and Computing, pages 913-924, 2018. doi: $10.1007 / \mathrm{s} 11517-018-1931-\mathrm{z}$

14. V. Groenhuis and S. Stramigioli. Rapid Prototyping High-Performance MR Safe Pneumatic Stepper Motors. IEEE/ASME Transactions on Mechatronics, 23(4):1843-1853, 2018. doi: 10.1109/TMECH.2018.2840682 
15. V. Groenhuis, F. J. F. Siepel, J. Veltman, J. K. J. van Zandwijk, and S. Stramigioli. Stormram 4: An MR Safe Robotic System for Breast Biopsy. Annals of Biomedical Engineering, 46(10):1686-1696, 2018. doi: 10.1007/s10439-018-2051-5

16. V. Groenhuis, F. Visentin, F. J. Siepel, B. M. Maris, D. Dall'alba, P. Fiorini, and S. Stramigioli. Analytical derivation of elasticity in breast phantoms for deformation tracking. International Journal of Computer Assisted Radiology and Surgery, 13(10):1641-1650, 2018

17. V. Groenhuis, J. Veltman, F. Siepel, and S. Stramigioli. Stormram 3: A Magnetic Resonance Imaging-Compatible Robotic System for Breast Biopsy. IEEE Robotics and Automation Magazine, 17(May):34-41, 2017. doi: 10.1109/MRA.2017.2680541

18. V. Groenhuis and S. Stramigioli. Laser-cutting pneumatics. IEEE/ASME Transactions on Mechatronics, 21(3):1604-1611, 2016. doi: 10.1109/TMECH. 2015.2508100

\section{Book chapters}

19. V. Groenhuis, J. Siepel, and S. Stramigioli. Sunram 5 : An MR safe robotic system for breast biopsy, driven by pneumatic stepper motors. In M. H. Abedin-Nasab, editor, Handbook of Robotic and Image-Guided Surgery, chapter 22, pages 375-396. Elsevier, 2019

\section{Patents}

20. V. Groenhuis, F. J. Siepel, and S. Stramigioli. Pneumatic Stepper Motor And Device Comprising At Least One Such Pneumatic Stepper Motor, 2018

\section{Submitted}

21. M.K. Welleweerd, F.J. Siepel, V.Groenhuis, J.Veltman, S.Stramigioli. Design of an end-effector for robotic assisted US guided breast biopsy. Submitted to the International Journal of Computer Assisted Radiology and Surgery (IJCARS), 2019

22. V. Groenhuis, A. Nikolaev, S.H.G. Nies, M.K. Welleweerd, L. de Jong, H.H.G. Hansen, F.J. Siepel, C.L. de Korte, S. Stramigioli. 3D Ultrasound Elastography Reconstruction Using Acoustically Transparent Pressure 
Sensor on Robotic Arm. Submitted to The 11th International Conference on Information Processing in Computer-Assisted Interventions (IPCAI), Munich, 2020

23. V. Groenhuis, F.J. Siepel, S. Stramigioli. Real-time Deformation Tracking System for Breast Biopsy. Submitted to The 11th International Conference on Information Processing in Computer-Assisted Interventions (IPCAI), Munich, 2020

24. V. Groenhuis, E. Tagliabue, M.K. Welleweerd, F.J. Siepel, J.D. Munoz Osorio, B.M. Maris, F. Allmendinger, D. Dall'Alba, P. Fiorini, S. Stramigioli. Deformation Compensation in Robotically-Assisted Breast Biopsy. Submitted to The 11th International Conference on Information Processing in Computer-Assisted Interventions (IPCAI), Munich, 2020 


\section{Contents}

I General Introduction 1

1 Introduction 3

1.1 Clinical background . . . . . . . . . . . . . . . 3

1.2 Main research question . . . . . . . . . . . . . . . . . 12

1.3 General approach . . . . . . . . . . . . . . . . . . . . 12

1.4 State of art . . . . . . . . . . . . . . . . . . . . . . . . . . . 13

1.5 Pneumatic stepper motors . . . . . . . . . . . . . . . . . 16

1.6 Stormram research line . . . . . . . . . . . . . . . . . 21

1.7 MURAB research line .................. 22

II Pneumatic stepper motors $\quad 25$

2 Laser-cutting Pneumatics 27

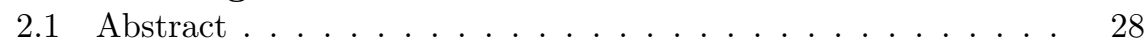

2.2 Introduction . . . . . . . . . . . . . . . . . . . . . . . . . . . 28

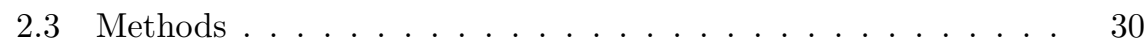

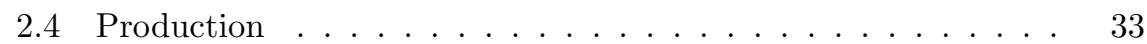

2.5 Valves . . . . . . . . . . . . . . . . . 37

2.6 Applications in a MRI-compatible robotic system . . . . . . . 39

2.7 Measurements . . . . . . . . . . . . . . . . . . . . . . . . . . . . . . .

2.8 Conclusion .......................... 44

3 Rapid Prototyping High-Performance MR Safe Pneumatic Stepper Motors 45

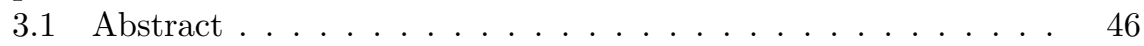

3.2 Introduction . . . . . . . . . . . . . . . . . . . . 46

3.3 Design and production . . . . . . . . . . . . 50 
3.4 Measurements .......................... 56

3.5 Conclusion ...................... 69

4 Dual-Speed MR Safe Pneumatic Stepper Motors 71

4.1 Abstract . . . . . . . . . . . . . . . 72

4.2 Introduction . . . . . . . . . . . . . . . . . . . . 72

4.3 Materials and Methods . . . . . . . . . . . . . . 75

4.4 Evaluation . . . . . . . . . . . . . . . . . . 79

4.5 Discussion . . . . . . . . . . . . . . . . . 83

4.6 Conclusion ..................... 86

5 Miniaturization of MR Safe Pneumatic Rotational Stepper $\begin{array}{lr}\text { Motors } & \mathbf{8 7}\end{array}$

5.1 Abstract ....................... 88

5.2 Introduction . . . . . . . . . . . . . . . . . . . . 88

5.3 Materials and Methods . . . . . . . . . . . . . . . . 90

5.4 Experimental Results and Analysis . . . . . . . . . . . . . 96

5.5 Conclusion . . . . . . . . . . . . . . . . . . 101

\section{MR safe robotic systems for breast biopsy 103}

6 Controlling the Stormram 2: An MRI-compatible Robotic Sys$\begin{array}{lr}\text { tem for Breast Biopsy } & 105\end{array}$

6.1 Abstract . . . . . . . . . . . . . . . 106

6.2 Introduction . . . . . . . . . . . . . . . . 106

6.3 Design and Implementation . . . . . . . . . . . . . . . . . . . . . . 108

6.4 Kinematics ....................... 111

6.5 Measurements . . . . . . . . . . . . . . . . . . . 118

6.6 Discussion . . . . . . . . . . . . . . . . . . . . . . . . . . . . . . 121

6.7 Conclusion .......................... 122

7 Stormram 3: An MRI-compatible robotic system for breast biopsy

7.1 Abstract . . . . . . . . . . . . . . . . . . . 124

7.2 Introduction . . . . . . . . . . . . . . . . . . 124

7.3 Design and implementation . . . . . . . . . . . . . . . 126

7.4 Kinematics and workspace . . . . . . . . . . . . . . . . 131

7.5 Measurements . . . . . . . . . . . . . . . . . . . 134

7.6 Discussion . . . . . . . . . . . . . . . . 136

7.7 Conclusion . . . . . . . . . . . . . . . . 138 
8 Design and Characterization of Stormram 4: An MRI-Compatible Robotic System for Breast Biopsy 139

8.1 Abstract . . . . . . . . . . . . . . . . . . . . 140

8.2 Introduction . . . . . . . . . . . . . . . . . . . . 140

8.3 Design and Implementation . . . . . . . . . . . . . . . . 142

8.4 Kinematics and Workspace . . . . . . . . . . . . . . . 145

8.5 Measurements and Results . . . . . . . . . . . . . . . . . . . . . 149

8.6 Discussion . . . . . . . . . . . . . . . . . . . . . . . 152

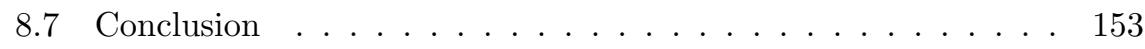

9 Stormram 4: An MR Safe Robotic System for Breast Biopsy 155

9.1 Abstract . . . . . . . . . . . . . . . . 156

9.2 Introduction . . . . . . . . . . . . . . . . 156

9.3 Materials and Methods . . . . . . . . . . . . . . . . . . . 159

9.4 Results . . . . . . . . . . . . . . . . . . . . . . . 168

9.5 Discussion . . . . . . . . . . . . . . . . . . . . . . 171

9.6 Acknowledgments . . . . . . . . . . . . . . . . . . . 173

10 Sunram 5: An MR safe robotic system for breast biopsy, driven by pneumatic stepper motors $\quad \mathbf{1 7 5}$

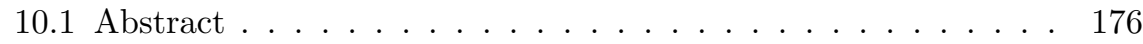

10.2 Introduction . . . . . . . . . . . . . . . . . . 176

10.3 Pneumatic cylinders . . . . . . . . . . . . . . . . . . . 184

10.4 Stepper motors . . . . . . . . . . . . . . . . . . . 188

10.5 Design of Sunram $5 \ldots \ldots \ldots$

10.6 Control of pneumatic devices _ . . . . . . . . . . . . 197

10.7 Evaluation of stepper motors and Stormram 4 . . . . . . . 201

10.8 Conclusion . . . . . . . . . . . . . . . . 207

IV MURAB / Tissue deformations $\quad 209$

11 End-effector design 211

11.1 Abstract . . . . . . . . . . . . . . . . . . . . 212

11.2 Introduction . . . . . . . . . . . . . . . . . . . 212

11.3 Method . . . . . . . . . . . . . . . . . . . . . 214

11.4 Results . . . . . . . . . . . . . . . . . . . 221

11.5 Discussion . . . . . . . . . . . . . . . . . . . . 223

11.6 Conclusion and recommendations . . . . . . . . . . . . 224 
12 Analytical derivation of elasticity in breast phantoms for deformation tracking

12.1 Abstract . . . . . . . . . . . . . . . . 226

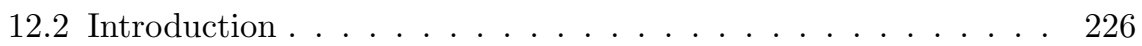

12.3 Materials and Methods . . . . . . . . . . . . . . . . . . 228

12.4 Elasticity estimation . . . . . . . . . . . . . . . . . . . 231

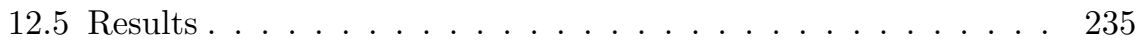

12.6 Discussion . . . . . . . . . . . . . . . . . 240

13 3D Ultrasound Elastography Reconstruction Using Acoustically Transparent Pressure Sensor on Robotic Arm 241

13.1 Purpose . . . . . . . . . . . . . . . . . . . . 242

13.2 Methods . . . . . . . . . . . . . . . . . . 243

13.3 Results. . . . . . . . . . . . . . . . . . . . . . . . . . . . . . . . 244

13.4 Conclusion .......................... 245

14 Real-time Deformation Tracking System for Breast Biopsy 247

14.1 Purpose . . . . . . . . . . . . . . . . . . . . 248

14.2 Methods . . . . . . . . . . . . . . . . . . . 248

14.3 Results. . . . . . . . . . . . . . . . . . . 250

14.4 Conclusion ..................... 250

15 Deformation Compensation in Robotically-Assisted Breast

Biopsy

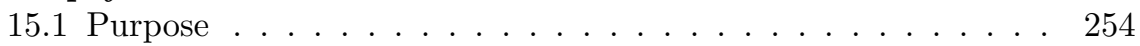

15.2 Methods . . . . . . . . . . . . . . . . . . . 254

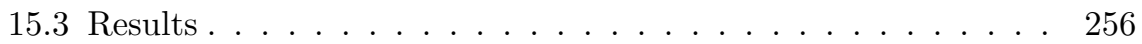

15.4 Conclusion . . . . . . . . . . . . . . 257

\section{General Discussion and Conclusion 259}

16 Discussion $\quad \mathbf{2 6 1}$

16.1 Scientific and technological discussions . . . . . . . . . . . . . 261

16.2 Accuracy of biopsy needle placement . . . . . . . . . . . . . 265

16.3 Procedure time . . . . . . . . . . . . . . . . . . . . . . . . . . . . . . . . . . 267

16.4 Conclusion . . . . . . . . . . . . . . . 267

$\begin{array}{ll}\text { Dankwoord } & 285\end{array}$

$\begin{array}{ll}\text { Biography } & 289\end{array}$ 


\section{Part I}

\section{General Introduction}





\section{INTRODUCTION}

The field of this thesis' research is in breast cancer screening and diagnosis, more specifically in the biopsy of MRI-visible lesions. This chapter starts with the clinical background, followed by the general approach split in two research lines. The chapter concludes by a short overview of the research projects conducted for this thesis.

\subsection{Clinical background}

This clinical background covers the anatomy of the breast, a few words about the breast cancer and an overview of techniques used in screening and treatment.

\subsubsection{Breast anatomy}

The breast is one specific organ of the human body. In females it generally develops to large sizes than in men, especially during puberty and pregnancy. The main function is lactating, i.e. to produce milk for infants.

Figure 1.1 shows the anatomy of the breast. It consists of several tissue types including skin, fibrous tissue (suspensory/Cooper ligaments), fatty tissue, lactiferous ducts (mammary/milk ducts), lobules, aerola with nipple, blood vessels, lymph nodes. The breast is adjacent to the pectoralis major muscle, which is in turn connected to the ribs. 


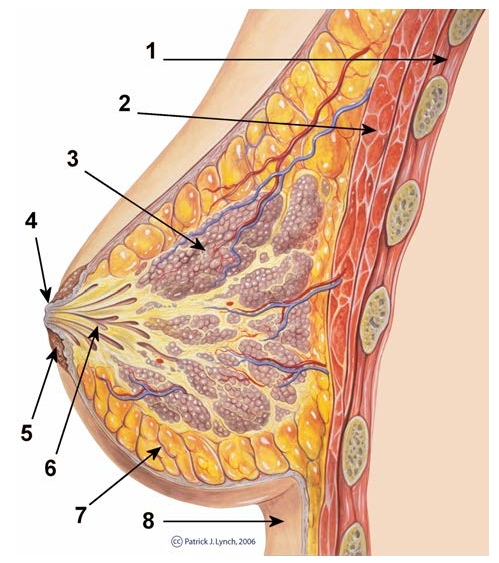

Figure 1.1: Anatomy of the human breast. 1: Chest wall, 2: Pectoralis muscles, 3: Lobules, 4: Nipple, 5: Aerola, 6: Milk duct, 7: Fatty tissue, 8: Skin. Image: Patrick J. Lynch / CC BY 3.0

\subsubsection{Breast cancer}

Like any organ, the breast can develop diseases which may need treatment. One particulary dangerous disease is cancer, which poses a siginificant health risk for the person if left untreated. Among females, breast cancer is the most commonly diagnosed cancer with about 2.1 million new cases in 2018 and the leading cause of cancer death worldwide [20]. Breast cancer has far-reaching personal consequences and therefore has been the focus of much research worldwide.

There are several types of breast cancer with different causes and origins. Common examples are ductal carcinoma (originating in milk ducts) and lobular carcinoma (originating in lobules). There are several subtypes depending on the characteristics of the cancerous cells, of which some are invasive while others do not spread out (e.g. ductal carcinoma in situ, or DCIS).

Several risk factors are known to increase the chances of developing breast cancer. Examples are having an unhealthy lifestyle such as smoking, obesity and excessive drinking of alcohol. Another important risk factor is in genetics, more specifically the BRCA1 and BRCA2 tumor-suppression genes which are hereditary and cause an elevated risk for development of breast cancer and ovarian cancer. Furthermore, women have a much higher risk than men and the risk also increases with age.

The development of breast cancer can be classified in five stages, in which stage 0 indicates a non-invasive cancer (e.g. DCIS), stage I to III indicate invasive breast cancers of increasing size and/or spreading within breast, and stage IV finally indicates invasive cancer which has spread out to other organs of the 
body. The five-year survival rate is relatively high in stage 0 and significantly decreases in the higher stages. This implies that early detection and treatment of breast cancer is crucial to maximize the life expectancy.

\subsubsection{Screening and treatment}

A periodic breast screening program has been set up in most countries, especially the developed ones with a high standard for healthcare. Taking the Netherlands as an example, the routine screening program involves taking a mammogram once in every two years for women aged 50-75 years. In case of elevated risk for breast cancer, abnormalities, unspecific complaints and/or other indications the mammography frequency can be increased and/or supplemented with additional imaging techniques. Suspicious lesions may need to be biopsied, which involves taking out a sample of the lesion for histological assessment. If cancerous tissue is detected then a treatment plan can be set up, which may involve radiation and/or surgical techniques.

\subsubsection{Imaging techniques}

Several two-dimensional and three-dimensional techniques are available to visualize the interior of the breast, allowing to search for suspicious lesions. Every imaging technique has its own advantages and disadvantages and a good understanding of these techniques is essential in obtaining an accurate diagnosis. The result of a scan can be classified according to the BI-RADS scheme. The general definition is as follows: 1 = normal, healthy tissue, $2=$ benign lesions only, 3 = probably benign but needs follow-up, $4=$ suspicious and needs additional exam, $5=$ probably malign, $6=$ proven malignancy [137].

\section{Mammography}
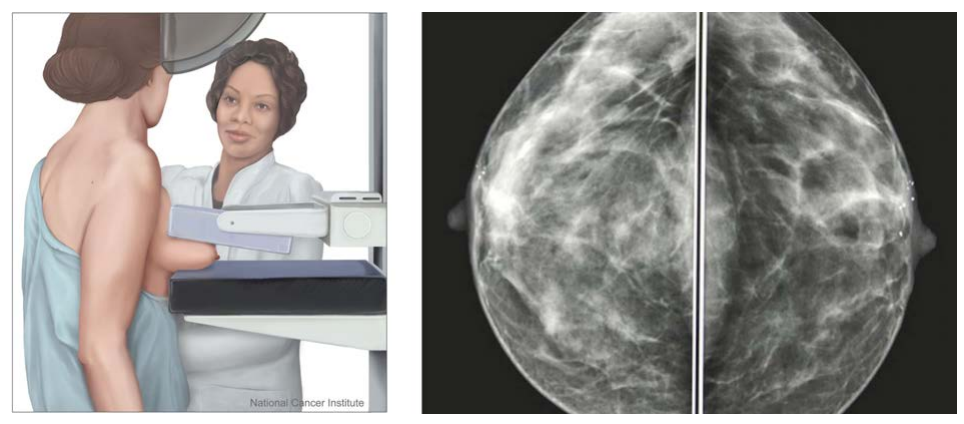

Figure 1.2: Left: Mammography imaging procedure. Right: Example mammogram. Source: National Cancer Institute. 
Mammography uses X-rays to create a two-dimensional black/white image of the breast. The breast is first compressed between two transparent plates (Figure 1.2(left)) and then a small dose of radiation is used to obtain the X-ray image. Several structures can be distinguished on the mammogram as shown in Figure 1.2(right). Denser tissue generally appears brighter than soft tissue. In many cases the radiologist is able to distinguish suspicious lesions such as bright spots and other abnormalities, especially if the breast is not too dense. If one or more lesions are found then an ultrasound exam is usually planned.

The mammogram results in a two-dimensional projection of the breast, making it difficult to distinguish structures that are overlapping in the direction perpendicular to the imaging plane. This is especially an issue in dense breasts, resulting in reduced sensitivity of suspicious lesions.

\section{Ultrasound}
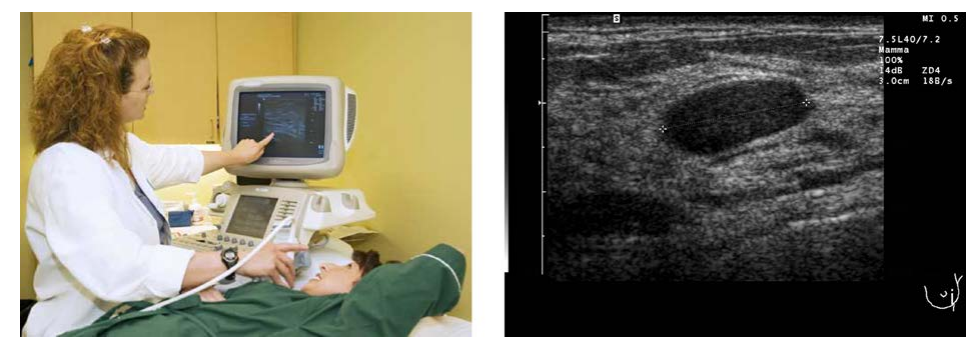

Figure 1.3: Left: Ultrasound scanning procedure. Right: Ultrasound scan exposing lesion in breast. Sources: RadiologyInfo.org, RadiologicTechnology.org

Ultrasound, also known as ultrasonography, uses acoustic waves with a frequency of several megahertz to image tissue in one plane. The acoustic waves are emitted from an array of piezo elements inside a handheld device, called the transducer. These waves are transmitted, attenuated and reflected inside tissue and at boundary layers between tissue types. The transducer picks up the reflected acoustic waves and reconstructs a two-dimensional image based on the shape and timing characteristics of the received waveforms in relation to the transmitted waveforms.

Figure 1.3(left) shows an impression of the procedure. The radiologist inspects the ultrasound images for abnormalities (lesions) while moving the probe around the whole breast. Figure 1.3(right) shows an example ultrasound scan in which a dark oval shape can be seen which the radiologist may classify as a suspicious lesion.

One important advantage of ultrasound over mammography is that the imaging plane can be almost arbitrarily chosen. This way it is possible to examine a suspicious section from different angles and assess its characteristics 
with respect to adjacent tissue in all directions. Another advantage is that ultrasound does not use ionizing radiation. A drawback is that the imaging depth is limited to a few centimetres, depending on the frequency of the transducer, and not all lesions can be detected on ultrasound.

If a suspicious lesion is found on ultrasound which is classified as BI-RADS 3 or higher then an ultrasound-guided biopsy is generally advised.

\section{MRI}
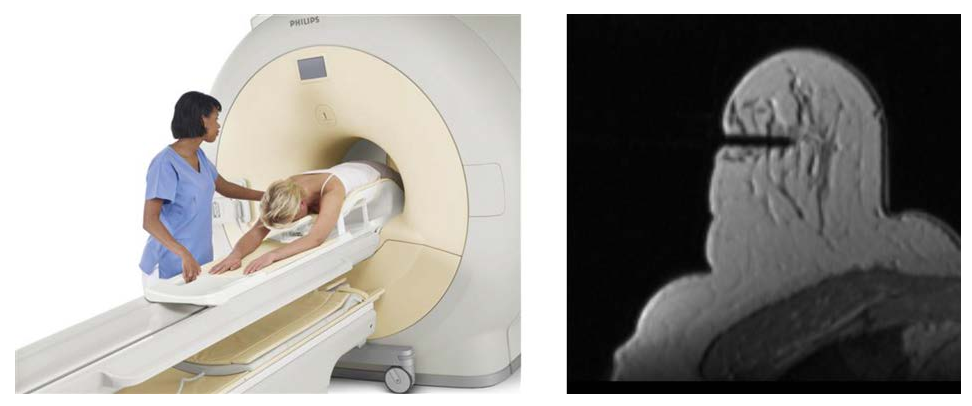

Figure 1.4: Left: Patient moving in an MRI scanner for a scan. Right: Confirmation scan during the biopsy procedure. Sources: MiddakotaClinic.com / RadboudUMC

Not all lesions can be found by mammography or ultrasound. MRI (magnetic resonance imaging) does not generate ionizing radiation and has the highest sensitivity among all imaging modalities, but is also one of the most expensive. A small set of patients may be screened in MRI: women with an elevated risk for breast cancer due to e.g. genetics, women with unspecified complaints and in case of a pre-operative scan.

The MRI scanner uses a strong magnetic field with oscillating gradients which resonate with protons (hydrogen atoms). In a uniform magnetic field the spin axes of all protons line up with this magnetic field. These spins can be deflected to a different alignment by superimposing an oscillating magnetic field on top of the uniform field, this is done by rapidly oscillating a set of electromagnetic coils. After turning off these oscillations the protons naturally fall back to the original state, aligning the spin with the uniform magnetic field again and hereby transmitting a radiofrequency (RF) wave. The time needed to transfer from the excited to original state is called the $\mathrm{T} 1$ relaxation time which is dependent on the type of tissue. Position information can be encoded by applying gradients to the magnetic field to define two-dimensional slices, and using phase and frequency encoding schemes to distinguish rows and columns within that slice.

Several acquisition sequences are possible by changing various parameters such as the repetition time (TR), echo time (TE) and others. Example se- 
quences are the T1-weighted spin echo which uses short TR and TE, while T2-weighted spin echo uses long TR and TE times. Another sequence is the balanced gradient echo sequence (3D Hyce on Esaote G-Scan Brio scanner).

In the MRI procedure the patient lies on a bed which is moved inside the MRI tunnel (Figure 1.4). Several scans are taken using different sequences. A contrast agent can be applied which emphasizes the blood vessels in the MRI scans, especially when compared with a pre-contrast scan. This is useful in detecting tumors as one characteric is angiogenesis, i.e. the formation of a blood vessel network in/around the tumor.

When a lesion is found which is classified as BI-RADS 3 or higher then a biopsy is generally advised. Biopsies can be done under ultrasound, stereotactic (x-ray) or MRI guidance. The ultrasound-guided biopsy procedure is the easiest and therefore the primary choice, but if the lesion is only detectable on MRI then a MRI-guided biopsy will be needed.

MR safety If a new device is developed for use inside the MRI scanner then special precautions must be followed to ensure safety of the patient. The ASTM F2503 standard defines three categories of MRI devices: MR safe, MR conditional and MR unsafe [114]. The MR safe requirement implies that the device is free of metallic, ferromagnetic and conductive materials and therefore inherently safe to use in all MRI scanners. This is regardless of the field strength and other parameters such as maximum gradients and minimum distance to patient. The MR conditional classification indicates that the device is only safe when certain given conditions are all met, while devices with the MR unsafe classification pose unacceptable risks and cannot be used in any MRI environment. This scheme replaces the former one (MR compatible/safe) which is known to cause confusion and errors: many "MRI compatible" devices were only tested under certain conditions and sometimes resulted in unsafe behaviour in other environments, leading to serious risks.

\section{Elastography}

Tumors have a higher stiffness than normal tissue. This allows to detect certain lesions by palpation (scanning the breast with the fingers checking for lumps under the skin) and/or by elastographic imaging. The sensitivity of palpation is limited to relatively large and/or superficial (i.e. close to the skin) masses only, so for deeper lesions some form of an elastographic imaging is needed.

Several elastography techniques can be used. Strain imaging, or quasistatic elastography, uses an external object (e.g. an ultrasound transducer) which is pressed against the breast with a known pressure (stress), introducing a displacement and local deformations of tissue (strain). The magnitude of these local deformations can be measured with an imaging technique (e.g. ultrasound) and the ratio between stress and strain is a measure for the elasticity. 


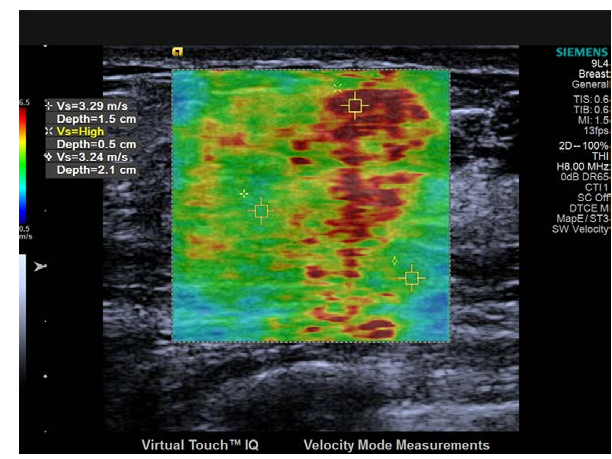

Figure 1.5: Example ultrasound elastography scan. Source: Siemens Healthcare

Instead of externally induced mechanical excitation, it is also possible to generate excitations internally, i.e. from within the organ. This is possible by using an ultrasonic focused beam which generates shear waves originating from the force region of excitation. The effect of these shear waves, in particular the propagation speed, can be related to the local stiffness of the internal tissue. This principle is used in the acoustic radiation force imaging (ARFI) and shear wave elasticity imaging (SWEI) techniques [13, 96].

Instead of ultrasound elastography it is also possible to use a different imaging modality such as MRI. In magnetic resonance elastography shear waves are generated by an external mechanical oscillator after which the velocity of the generated shear waves are measured by a special MRI scanning sequence.

\section{Other techniques: CT, PET, SPECT, PAMMO}

Where a mammography takes a single X-ray image, computed tomography (CT) takes multiple X-rays from different angles allowing to reconstruct a threedimensional scan of the breast. Positron emission tomography (PET) and single-photon emission computed tomography (SPECT) also reconstruct threedimensional scans by detecting gamma rays emitted by radioactive tracers and visualizing the stream and uptake of fluids inside the body. The drawback of CT is that the radiation dose is higher than that of a single mammography, and the equipment is bigger and more complicated. PET and SPECT also involve ionizing radiation that may be harmful and the scanning procedure is relatively slow.

In elastography one of the newest techniques being researched is photoacoustic imaging, or pammography. This involves firing laser pulses inside the breast to trigger localized acoustic waves, predominantly originating from areas of high laser energy uptake. The acoustic waves are recorded by arrays of ultrasonic detectors from which a three-dimensional elastography model is 
reconstructed. Photoacoustic imaging does not involve ionizing radiation, but the use of technology has not yet evolved to everyday's clinical practice.

\subsubsection{Breast biopsy}

The use of imaging techniques may result in the detection of one or more suspicious lesions that need to be further investigated. The golden standard in accurate histological evaluation is the biopsy. A biopsy involves inserting a hollow needle towards the lesion, followed by a firing sequence in which a small sample of the lesion is cut off and encapsulated inside the needle and later extracted. The sample(s) are examined for malignancy after which the radiologist can decide on the next steps depending on the outcome.

It is of crucial importance that the biopsy sample includes part of the suspicious lesion. If the lesion is missed then the biopsy needs to be re-done, resulting in additional tissue damage and a longer procedure time, to avoid the possibility of a false negative outcome.

In order to bring the needle towards the lesion, the location of the lesion must be known and the path of the needle must be controlled such that it goes to that location. Several techniques are available in the current clinical workflow, which usually involves ultrasound, stereotactic (X-ray) or MRI.

\section{Ultrasound-guided biopsy}
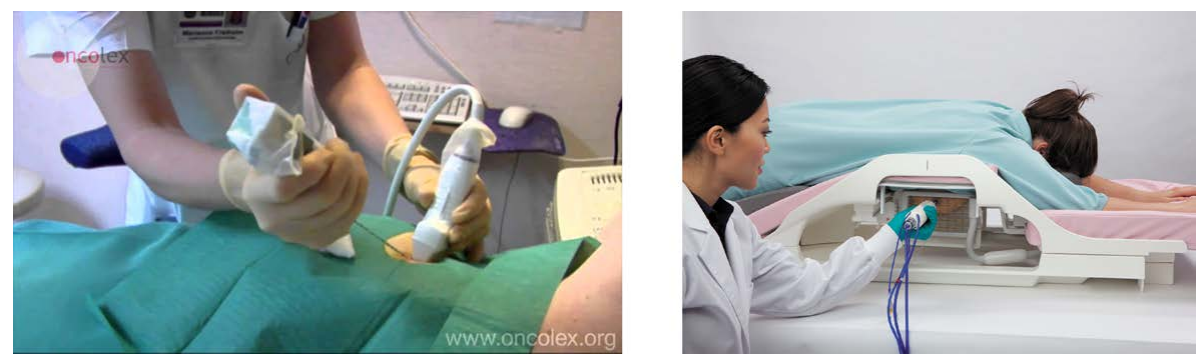

Figure 1.6: Left: Ultrasound-guided breast biopsy procedure. Right: MRI-guided biopsy procedure. Sources: Oncolex.org, InvivoCorp.com

Figure 1.6(left) shows the ultrasound biopsy procedure. After applying local anaesthetics an ultrasound transducer is positioned on the breast by one hand with the lesion in view of the transducer, while the other hand inserts the biopsy needle. The needle is angulated manually such that the path of the needle goes towards the lesion as observed on the real-time ultrasound image. Upon reaching the lesion the biopsy gun is fired manually and the biopsy needle is extracted. 


\section{MRI-guided biopsy}

If ultrasound-guided biopsy is infeasible due to e.g. invisibility of the lesion on ultrasound then a MRI-guided biopsy may be necessary. Figure 1.6(right) shows the setup containing a patient bed with a hole for the breast. The breast is immobilized by two vertical plates of which one contains a rectangular grid. The patient is first scanned in MRI (without and with contrast agent) and the suspicious lesion is localized and selected. The biopsy software then calculates and displays the required grid position and needle insertion depth in order to target this lesion. The patient is moved out of the scanner and a stylet through a sheath is inserted to create access to the lesion. The stylet is replaced by an obturator and the patient is scanned again to confirm that the location of the tip coincides with that of the lesion. If not then the last steps are repeated until the tip is at the right site. The patient is then moved again out of the scanner and the biopsy needle is inserted, usually taking multiple samples under vacuum assistance. A localization clip is inserted and a final confirmatory scan is taken.

The whole MRI-guided biopsy procedure takes about 45 to 60 minutes. A relatively thick $(4 \mathrm{~mm})$ biopsy needle is used which allows to take approximately ten samples and transport these through the needle to a container under vacuum assistance.

Analysis of current MRI-guided biopsy procedure The current manual MRI-guided biopsy procedure requires the patient to move in and out of the scanner multiple times. The lesion is localized after a scan inside the scanner, but the needle can only be inserted outside the scanner by the radiologist. Although the breast is squeezed inbetween two plates, the lesion may still move due to breathing, involuntary muscle contractions and/or needle-tissue interaction. Movements of several millimetres have been measured in practice. The radiologist cannot compensate for these movements during needle insertion, only the next confirmatory scan allows to correct the projected path.

If a grid system is used then there is a discretization error involved due to the spacing of the grid. The grid size is typically in the order of $5 \mathrm{~mm}$ resulting in a maximum discretization error of approx. $3 \mathrm{~mm}$. This is in the same order as the size of the smallest lesions to be biopsied (order of $5 \mathrm{~mm}$ ). A post-pillar system is also available in certain MRI-guided biopsy systems, but this is rarely used by radiologists due to the additional complexity involved in manually adjusting the position and angle of the post-pillar system.

The aforementioned inaccuracies more or less force the radiologist to take away a relatively large volume of tissue samples. The vacuum-assited biopsy device is an effective tool for this, but the thick needle results in significant tissue damage.

The length of the MRI-guided biopsy procedure (45-60 min) combined with 
the relatively high tissue damage makes the manual MRI-guided biopsy procedure inaccurate and inefficient. There is a need for a more accurate and efficient way of performing biopsies of the lesions involved.

There are fundamental limitations in manual biopsy procedures. The space inside the MRI scanner is confined (especially in closed-bore, tunnel-type MRI scanners), so it is difficult for the radiologist to insert the needle inside the MRI scanner under visual guidance. A needle guide is required to reach specific coordinates, but such a guide is unable to re-position itself in real-time to correct the insertion path, unless a robotic system is used.

\subsection{Main research question}

The main research question is formulated as follows:

Could a robotic system improve accuracy and efficiency of biopsies of MRIvisible lesions in the breast?

On the clinical side, this research question limits the scope to biopsy of lesions in the breast that are only visible on MRI and not with other imaging techniques. On the technology side, it states the use of a robotic system which performs or guides the biopsy procedure. In terms of evaluation, the accuracy can be seen as the distance of the needle tip from the target during biopsy, while the efficiency refers to the required amount of tissue extracted and the total duration and cost of the procedure.

\subsection{General approach}

There are two distinct ways in which a robotic system can help in MRI-guided breast biopsy. The first category is by involving a robotic system inside the MRI scanner, and the second category involves a robotic system outside the MRI scanner. Both approaches are briefly explained in this section and form the two main pillars of the research in this thesis.

\subsubsection{Robotic system inside MRI scanner (Stormram)}

The most direct way to involve robotics in MRI-guided breast biopsy is to place a robotic breast biopsy system inside the MRI scanner. Such a robotic system can steer the biopsy needle to the desired target lesion under (near-)realtime MRI guidance and perform the biopsy immediately after confirmation of precisely hitting the target. One important challenge in this approach is the MRI compatibility requirement for all components of such a system, severily limiting the possible choices of construction materials and actuation methods. This approach has led to the development of five robotic systems, called Stormram 1 to 
4 and Sunram 5. Pneumatic stepper motors have been chosen as the actuation method for these robotic systems.

\subsubsection{Robotic system outside MRI scanner (MURAB)}

A robotic system outside the MRI scanner is also able to steer the biopsy needle to a given target location. The main challenge in this approach is the deformability of the breast which has the consequence that the location of the target lesion respective to the robot is variable. The approach of the MURAB (MRI and Ultrasound Robotic Assisted Biopsy) project is to involve additional imaging modalities such as ultrasound and stereo vision in order to quantitatively measure and/or predict the deformations of the breast, allowing to indirectly track the location of the lesion to be biopsied.

\subsection{State of art}

The challenge of precisely targeting MRI-visible lesions is not new. Much effort has been put in researching systems that can target lesions in a better way than the current clinical procedure. Several systems are designed for prostate biopsy and robotics play an important role in the majority of these systems.

Two distinct groups of state-of-art research can be distinguished. The first group utilizes a (robotic or manual) system inside the MRI scanner, whereas the second group present methods to target lesions using systems outside the MRI scanner. ${ }^{1}$

\subsubsection{MR safe and MR conditional actuation methods}

The MR safe/conditional requirement implies that conventional electromagnetic motors cannot be directly used in actuation of any MR robot. Several alternative actuation methods have been proposed and demonstrated:

- Piezo motors and ultrasonic motors, they are electric motors that only cause limited interference with the MRI's magnetic field. Using dedicated control electronics and taking certain precautions, such motors may be classified MR conditional and may be usable in actuation of MRI robots $[70,123,125]$. A drawback is that piezo/ultrasonic motors cannot be classified MR safe due to the use of electricity and metallic materials, so the MRI safety and imaging quality aspects have to be re-evaluated each time when operating conditions are expanded.

\footnotetext{
${ }^{1}$ Parts of this section have been reproduced from the introduction of Chapter 10 .
} 
- Bowden cables transport energy via solid wires guided through tubes $[24,70]$. Instead of tubes a system of pulleys can also be used. These techniques allow to place conventional motors away from the robot (outside the Faraday cage of the MRI scanner). If the wires and Bowden tubes (or pulleys) are made of non-metallic materials, the system could be made MR safe. Friction, backlash and elasticity in the rigid materials may make an effective energy transfer difficult, especially when many bends are present in the transmission line.

- Pneumatics use clean air as energy transfer medium which is abundant in hospitals and laboratory environments. As small leakages are acceptable, pneumatic cylinders can be manufactured using rapid prototyping techniques. Important limitations are the compressibility of the medium which makes precise position control of a single cylinder difficult $[41,135]$, and also the long distance between the (MR unsafe) controller manifold and robot leads to long pneumatic lines which results in relatively low bandwidth.

- Hydraulics make use of liquid to deliver power to the robotic system [78, 133]. The liquid is kept in a closed system with compressor and valves and leaks are to be avoided. A hydraulic device requires the use of precisely engineered components, which makes rapid prototyping relatively difficult compared to other techniques.

- Actuation by magnetic spheres driven by gradients of the MRI scanner have also been demonstrated [40]. This technique is relatively complicated as it requires precise control of the MRI's gradients while at the same time mitigating the imaging artifacts induced by the magnets.

- Shaped memory alloy (SMA) actuators generate unidirectional movements when heat is applied to a SMA spring. The heat can be generated by applying current through the SMA spring, of which the self-resistance results in resistive heating. Bidirectional movement is generated using complementary pairs of SMA springs [67]. The use of metallic materials in the SMA actuators and the application of current through it make the SMA actuators MR-conditional at best.

The author uses pneumatics as the energy transfer method in the form of pneumatic stepper motors. We show that fast and precise control is possible despite the low bandwidth and lack of direct position feedback.

\subsubsection{MR safe and MR conditional robotic systems}

Many MRI surgical robots have been developed in the past by various research groups. In this chapter a selection of robotic systems driven by pneumatic 

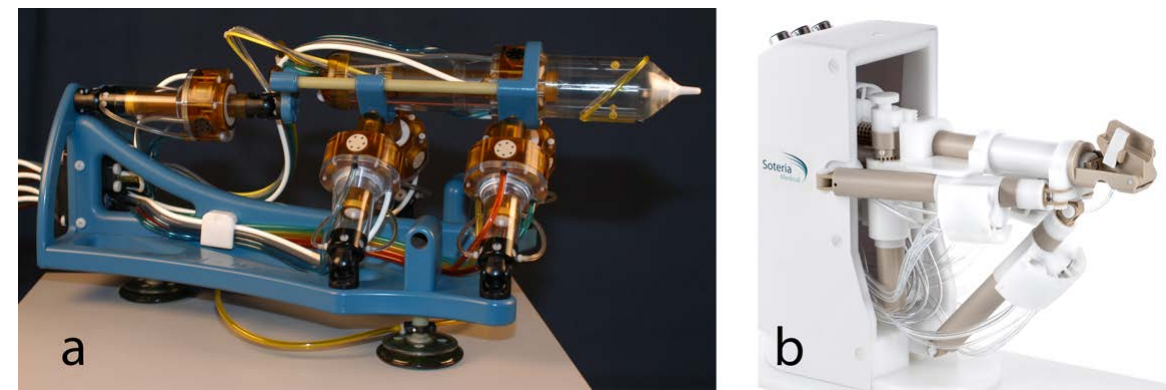

Figure 1.7: Two state-of-art MRI manipulators driven by pneumatic stepper motors. a) MrBot by Stoianovici et al. [120]. b) Soteria Remote Controlled Manipulator (RCM) by Bomers et al. [15].
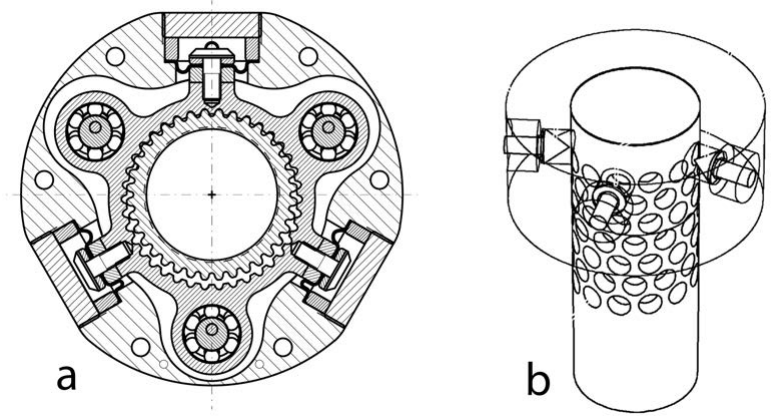

Figure 1.8: Pneumatic motors used to actuate the corresponding manipulators in Figure 1.7. a) PneuStep by Stoianovici et al. [118], b) Pneumatic stepper motor by Bomers et al. [15].

stepper motors is discussed: first three robots by other research groups and then five robots by the authors of this chapter.

\section{Pneumatic MRI robots by Stoianovici, Bomers and Sajima}

Stoianovici et al. developed several MRI robots for prostate biopsy. One example is the MrBot, shown in Figure 1.7(a) [120]. It is driven by six PneuStep rotational stepper motors of which a schematic cross section is shown in Figure 1.8(a). The PneuStep motor consists of three diaphragm cylinders that are connected to an internal gear. By alternatingly pressurizing the three cylinders, the internal gear is translated along a circular trajectory and its hoop gear in turn engages a spur gear. A leadscrew mechanism then converts the rotational motion of the spur gear into linear motion, resulting in movement of the robotic system. PneuStep makes use of optical positional encoders to detect and correct for missing steps, allowing to operate it at higher stepping 
speeds when less than maximum torque is needed. The valve manifold is put inside a shielded enclosure within the MRI room, allowing to reduce the tube lengths to a minimum [118].

The Soterial Remote Controlled Manipulator (RCM) by Bomers et al. is shown in Figure 1.7(b). Like MrBot, this robot is designed for prostate interventions [15]. It is driven by five pneumatic stepper motors of which a schematic drawing is shown in Figure 1.8(b). Its five cylinders have cone tips mounted on the pistons which engage on a two-dimensional pattern of holes on the rod. Pressurization of one cylinder pushes the associated cone tip into one hole, forcing the hole to align with the cone tip by the associated wedge mechanism and hereby introducing a displacement. Sequential pressurization of the right combination of cylinders result in either a screw movement or a linear movement of the rod, resulting in a small or large displacement of the robot linkages. The cylinders are double-acting, a single tube is used for the return stroke of all five pistons so that six tubes are used per actuator [15].

Sajima et al. developed a manipulator driven by rotational stepper motors [110]. Each stepper motor consists of three single-acting cylinders that act on a rotation gear by means of a wedge mechanism. By sequentially pressurizing the three cylinders the gear is driven around in either direction. In this design the gears have to be back-driveable in order to allow retraction of the pistons in the non-pressurized cylinders for continuous movements. A leadscrew finally converts the rotational motion of the gear into linear motion of the manipulator linkages.

An important limitation of the design of Sajima is that the wedge mechanism must be back-driveable due to the use of single-acting cylinders. This implies that the teeth cannot have sharp angles and significant torque is lost by friction of the sliding surfaces. On the other hand, Sajima's design is relatively compact and easy to manufacture compared to the designs of Stoianovici and Bomers.

The low nominal stepping frequency resulting from the long pneumatic tubes is an issue which has to be adressed in order to achieve both high speed and high accuracy. Stoinanovici's design utilizes position encoders which allow to speed up the motors when less than maximum torque is needed, while Bomers' design uses a 2-D hole pattern that enables both large and small actuation steps.

\subsection{Pneumatic stepper motors}

One of the approaches to tackle the clinical challenge is to use a MR safe robotic system inside the MRI scanner. Such a system needs to be actuated by means of a technology that does not make use of metallic, magnetic or conductive materials. Pneumatic stepper motors was chosen as the actuation mechanism 
for these systems. This chapter describes the development of these stepper motors.

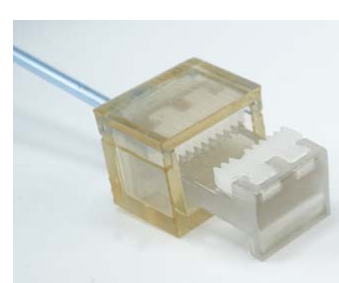

a

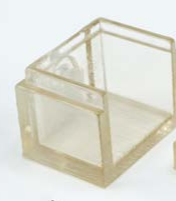

b

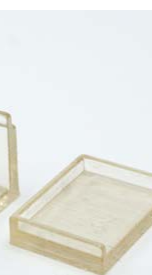

C

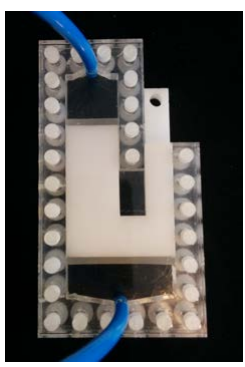

Figure 1.9: Left: Single-acting cylinder with individual components. Right: Double-acting cylinder.

A pneumatic stepper motor consists of two or three pneumatic cylinders. Each cylinder consists of a movable piston inside a cavity. In a double-acting cylinder there are two chambers on opposite ends of the piston, sealed off by a seal. By pressurizing either chamber there is a force exerted on the piston which then moves to the non-pressurized side.

Figure 1.9 shows an example single-acting and a double-acting cylinder. The single-acting cylinder is 3 -D printed, while the double-acting cylinder is constructed by laser-cutting parts and stacking these together using nylon screws. The cylinder bore(s) of both cylinders have a rectangular cross-section, which is characteristic for all pneumatic cylinders developed in this research. The double-acting cylinder is further explained in Chapter 2.

Figure 1.10 shows a selection of sixteen different pneumatic stepper motors developed and published during the PhD thesis. Figure 1.11 shows nine more pneumatic motors. All of these are manufactured by rapid prototyping techniques (3-D printing and/or laser-cutting). The motors can be classified as follows:

- Stepper or continuously rotating. Three motors are continuously rotating motors, all others are stepper motors.

- Linear, rotational or curved. The T-xx types are linear motors moving in a straight line, R-xx types are rotational motors that drive a shaft and $\mathrm{C}$-xx types are curved stepper motors which have a curved rack revolving around an imaginary axis.

- Mechanical transfer type. The majority of the motors employ teeth in the pistons that engage with a rach or gear, three use a dual slotted link mechanism (Bourke engine) and one uses a traditinoal crankshaft. 


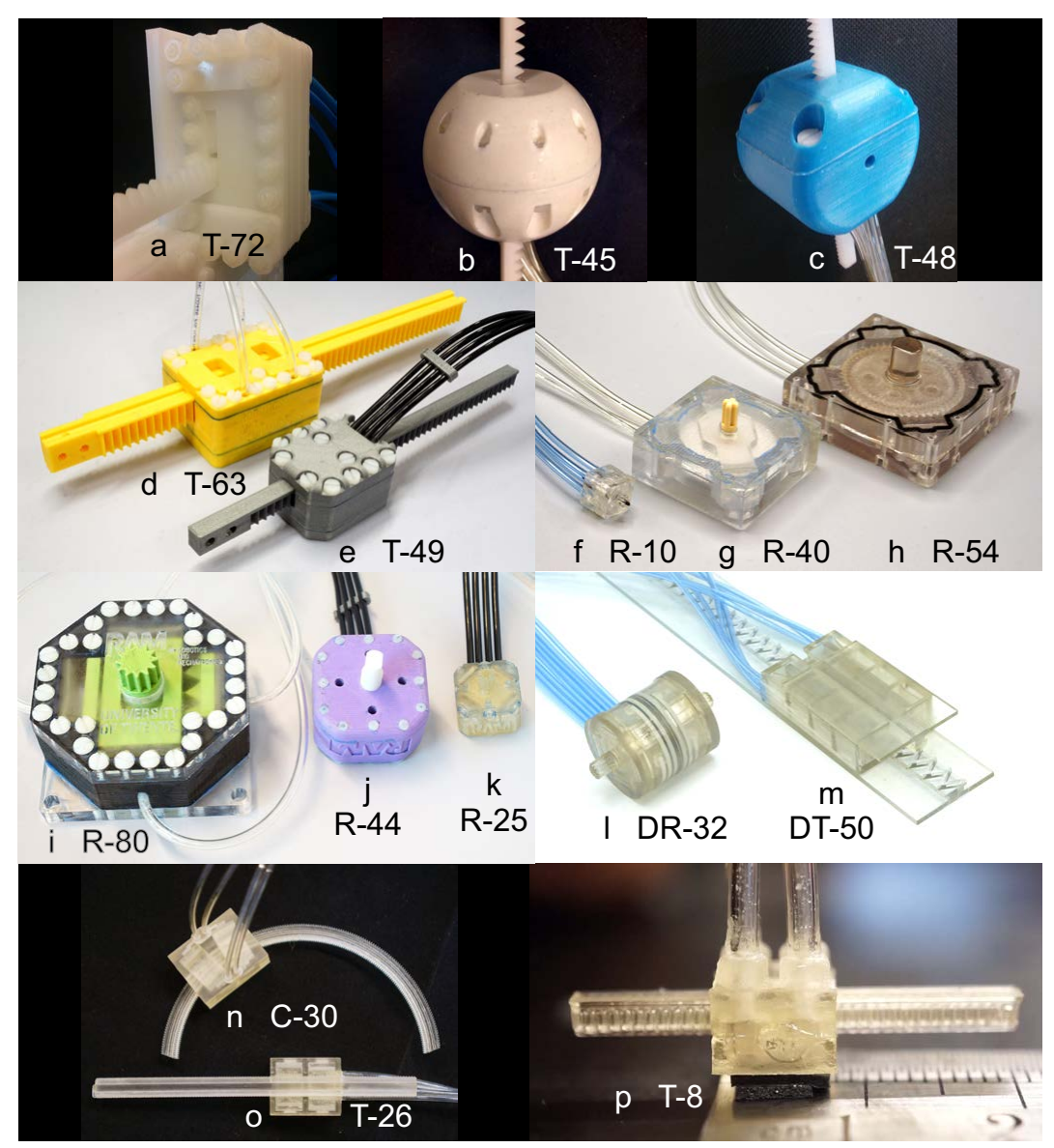

Figure 1.10: Selection of stepper motors developed in this research.

- Size and power. Stepper motors have been designed in sizes from $8 \mathrm{~mm}$ to $81 \mathrm{~mm}$. The number in the motor's name indicates the largest dimension. The motor size is strongly related to output force/torque and power, with bigger size motors delivering higher output.

- Step size: motors with fine-pitched teeth generally have smaller step sizes than those with coarse teeth or toothless types. The use of gears integrated in the motor further reduces the step size.

- Single or dual speed. The Dx-xx types are dual-speed motors which consist of two independent sub-motors with different step sizes, allowing to combine high speed with small step sizes. All other motors are single- 


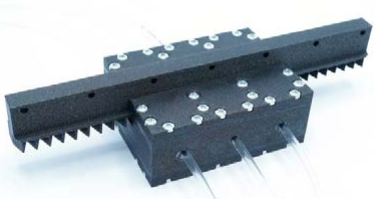

a. T-84

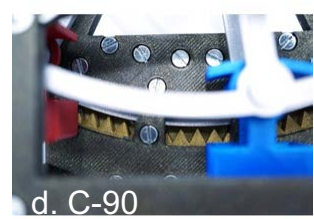

g. R-52

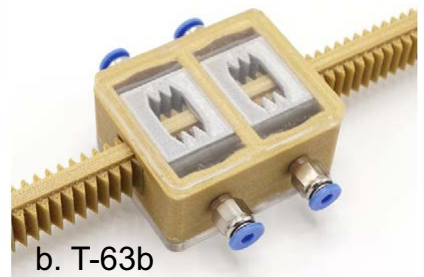

c. T-48b

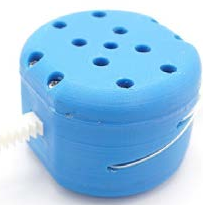

f. $R-64$

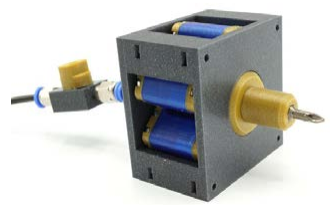

h. R-66
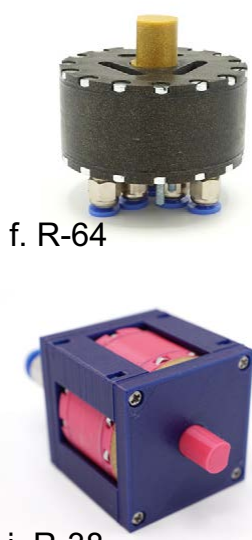

i. $\mathrm{R}-38$

Figure 1.11: Selection of additional pneumatic motors developed.

speed motors.

- Number of cylinders: the motors have two to five cylinders. In stepper motors the number of cylinders directly relates to the number of pneumatic lines (two per cylinder) and it also influences the control strategy and step size.

- Serviceability: certain motors are assembled using screws and can be opened for maintenance, other motors are glued together for compactness and cannot be serviced.

The design and evaluation of several motors are described in Part II and III, more specifically the following chapters:

- Chapter 2: T-72

- Chapter 3: T-63, T-49, R-80, R-44, R-25

- Chapter 4: DT-50, DR-32

- Chapter 5: R-10, R-40, R-54 
- Chapter 6: T-45

- Chapter 7: T-48, T-49

- Chapter 8-9: T-26, C-30

Table 1.1: Characteristics of developed pneumatic motors

\begin{tabular}{|c|c|c|c|c|c|}
\hline Name & Size $(\mathrm{mm})$ & Step & Force/Torque & Cylinders & $\begin{array}{l}\text { Piston } \\
\left(\mathrm{mm}^{2}\right)\end{array}$ \\
\hline T-08 & $8 \times 8 \times 5.5$ & $0.2 \mathrm{~mm}$ & & 2 & $2.5 \times 2.5$ \\
\hline $\mathrm{T}-15$ & $15 \times 15 \times 11$ & $0.25 \mathrm{~mm}$ & & 2 & $4 \times 4$ \\
\hline $\mathrm{T}-20$ & $20 \times 20 \times 13$ & $0.3 \mathrm{~mm}$ & & 2 & $7 \times 7$ \\
\hline $\mathrm{T}-26$ & $26 \times 21 \times 16$ & $0.25 \mathrm{~mm}$ & $63 \mathrm{~N}$ & 2 & $10 \times 10$ \\
\hline $\mathrm{T}-29$ & $29 \times 25 \times 19$ & $0.25 \mathrm{~mm}$ & & 2 & $10 \times 10$ \\
\hline $\mathrm{T}-32$ & $32 \times 30 \times 16$ & $0.3 \mathrm{~mm}$ & & 2 & $12 \times 10$ \\
\hline $\mathrm{T}-48$ & $48 \times 44 \times 34$ & $0.67 \mathrm{~mm}$ & $20 \mathrm{~N}$ & 3 & $12 \times 5$ \\
\hline $\mathrm{T}-48 \mathrm{~B}$ & $48 \times 44 \times 34$ & $0.67 \mathrm{~mm}$ & & 3 & $20 x 8$ \\
\hline T-49 & $49 \times 40 \times 31$ & $1.0 \mathrm{~mm}$ & $100 \mathrm{~N}$ & 2 & $14 \times 14$ \\
\hline T-63 & $63 \times 52 \times 36$ & $1.0 \mathrm{~mm}$ & $330 \mathrm{~N}$ & 2 & $20 \times 20$ \\
\hline T-63B & $63 \times 52 \times 36$ & $1.0 \mathrm{~mm}$ & $570 \mathrm{~N}$ & 2 & $24 \times 24$ \\
\hline $\mathrm{T}-84$ & $84 \times 64 \times 32$ & $2.0 \mathrm{~mm}$ & & 3 & $20 \times 20$ \\
\hline $\mathrm{R}-10$ & $10 \times 10 \times 10$ & $12.9^{\circ}$ & $0.0012 \mathrm{Nm}$ & 2 & $3 \times 4$ \\
\hline $\mathrm{R}-25$ & $25 \times 25 \times 20$ & $6.9^{\circ}$ & $0.10 \mathrm{Nm}$ & 2 & $10 \times 10$ \\
\hline $\mathrm{R}-25 \mathrm{~B}$ & $25 \times 25 \times 20$ & $10^{\circ}$ & & 2 & $10 \times 10$ \\
\hline $\mathrm{R}-38$ & $38 \times 38 \times 48$ & $\infty$ & & 2 & $12 \times 16$ \\
\hline $\mathrm{R}-40$ & $40 \times 40 \times 16$ & $1.01^{\circ}$ & $0.47 \mathrm{Nm}$ & 2 & $10 \times 10$ \\
\hline R-44 & $44 \times 44 \times 31$ & $10^{\circ}$ & $0.45 \mathrm{Nm}$ & 2 & $14 \times 14$ \\
\hline $\mathrm{R}-52$ & $52 \times 52 \times 36$ & $\infty$ & & 2 & $18 \times 18$ \\
\hline R-54 & $54 \times 54 \times 16$ & $0.00101^{\circ}$ & $0.24 \mathrm{Nm}$ & 2 & $14 \times 10$ \\
\hline R-64 & $64 \times 64 \times 32$ & $15^{\circ} / 7.5^{\circ}$ & & 3 & $24 \times 24$ \\
\hline $\mathrm{R}-66$ & $66 \times 66 \times 43$ & $\infty$ & & 5 & $14 \times 24$ \\
\hline $\mathrm{R}-80$ & $80 \times 80 \times 37$ & $10^{\circ}$ & $3.7 \mathrm{Nm}$ & 2 & $30 \times 20$ \\
\hline R-90 & $90 \times 90 \times 32$ & $90^{\circ}$ & $1.6 \mathrm{Nm}$ & 2 & $30 \times 20$ \\
\hline $\mathrm{C}-30$ & $30 \times 23 \times 14$ & $0.25^{\circ}$ & & 2 & $10 \times 10$ \\
\hline C-30B & $30 \times 27 \times 14$ & $0.5^{\circ}$ & & 2 & $10 \times 10$ \\
\hline C-90 & $90 \times 48 \times 32$ & $1^{\circ}$ & & 3 & $20 \times 20$ \\
\hline DT-50 & $50 \times 32 \times 14$ & $1.7+0.3 \mathrm{~mm}$ & $24 \mathrm{~N}$ & 4 & $10 \times 10$ \\
\hline DR-32 & $30 \times 30 \times 32$ & $10^{\circ}+12.9^{\circ}$ & $0.074 \mathrm{Nm}$ & 4 & $10 \times 10$ \\
\hline
\end{tabular}

Table 1.1 lists several characteristics of all developed motors. An up-to-date table with links to downloadable models is available in [44]. 


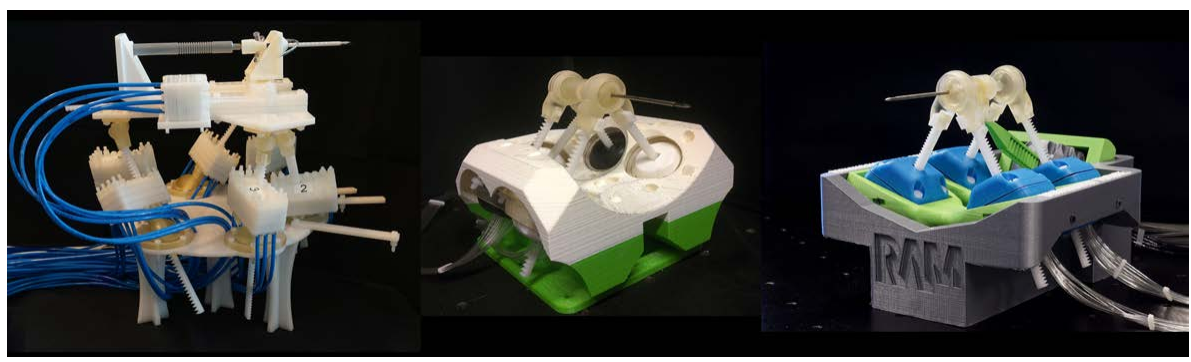

Figure 1.12: Left to right: Stormram 1, 2 and 3.

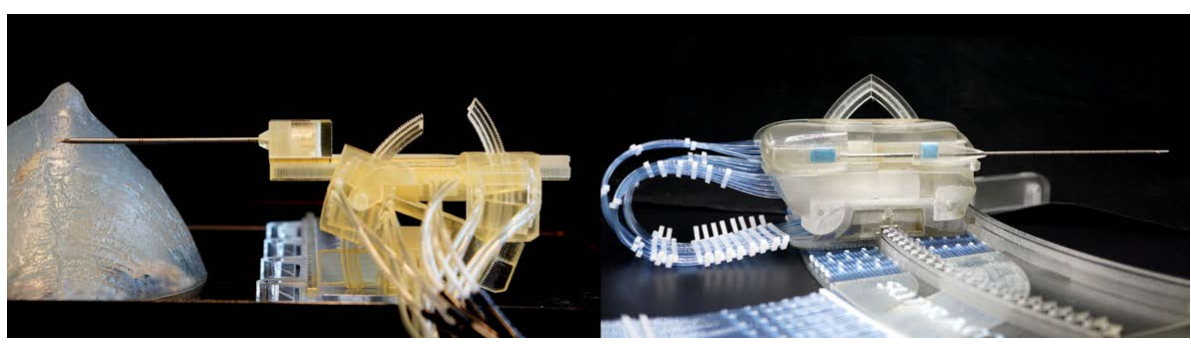

Figure 1.13: Stormram 4 (left) and Sunram 5.

\subsection{Stormram research line: robotic systems in- side MRI scanner}

The pneumatic stepper motor technology described in Section 1.5 can be used to actuate MR safe robotic manipulators.

Five iterations have been developed in this research line, called Stormram 1, 2, 3, 4 and Sunram 5. All these iterations are rapid prototyped by $3-\mathrm{D}$ printing and laser-cutting and actuated by pneumatic stepper motors, and are therefore inherently MR safe. (The biopsy needle is seen as a separate part of which the development is outside the scope of the Stormram project, and therefore does not need to be MR safe.)

The Stormram 1, 2 and 3 robotic systems are shown in Figure 1.12. All three of them are parallel manipulators. The different MR safe biopsy robots are described in Part II, more specifically in the following chapters:

Stormram 1 is described in Chapter 2. The kinematic system is based on a Stewart platform driven by six stepper motors. On top of the platform is a seventh actuator to ove the needle longitudinally towards the target. The resulting system is relatively large, with dimensions around $300 \mathrm{~mm}$ in all directions.

Stormram 2 is described in Chapter 6 and Stormram 3 in Chapter 7.

Chapters 8 and 9 describe the Stormram 4, and Chapter 10 finally describes 

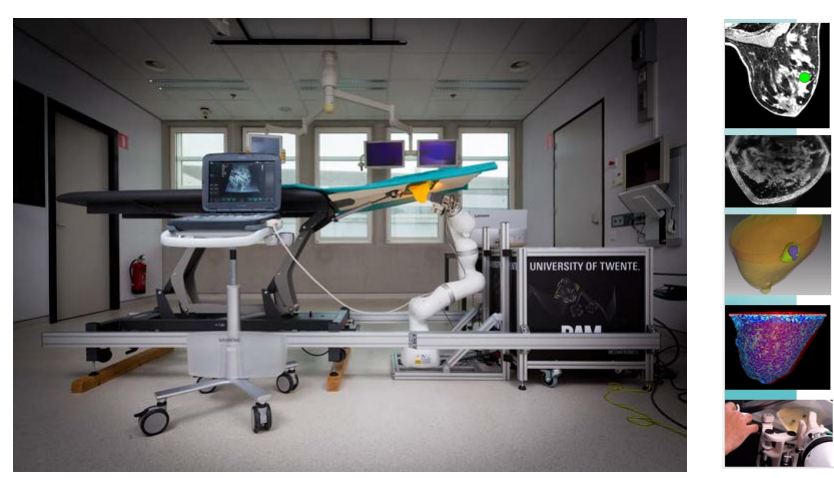

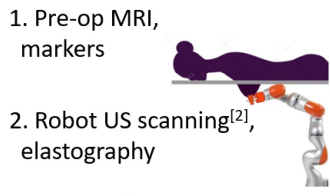

3. MRI-US fusion

4. FEM modeling simulations ${ }^{[1]}$

5. Biopsy

MURABproject.eu

Figure 1.14: Left: MURAB setup. Right: Simplified MURAB workflow. Setup photo by Gijs van Ouwerkerk / Siemens Healthineers.

the Sunram 5 robot.

\subsection{MURAB research line: robotic system out- side MRI scanner}

The MRI and Ultrasound Robotic Assisted Biopsy (MURAB) project combines different imaging modalities and techniques into a robotic system to solve the clinical challenge of performing biopsies of difficult lesions in the breast. The main difference with the Stormram project is that the MURAB robot arm is operated outside the MRI scanner and combines multiple imaging modalities including ultrasound, MRI, computer vision and elastography.

Figure 1.14(left) shows the overall setup of the MURAB system. It consists of a patient bed with a hole for the breast, a robotic arm with end-effector, an ultrasound system and a computer with interface.

Figure 1.14(right) shows the workflow. The patient is first scanned in the MRI with multimodality markers attached to the breast. Next, the patient is placed on the bed of the MURAB system and the location of the breast is registered using the stereo camera and computer vision. The ultrasound probe then scans the breast, collecting 2-D scans which are reconstructed in 3-D. The 3 -D ultrasound scan is registered to the MRI scan. Elastography data is also acquired, allowing to perform FEM simulations and SLAM-like algorithms. Finally the biopsy itself is planned and executed.

The chapters in Part IV describe several aspects of the MURAB system in detail. Chapter 11 describes the design and evaluation of the end-effector on the robot arm.

Chapters 12 and 13 both describe different techniques to estimate the elas- 
ticity of breast phantoms. The first technique analyzes deformations induced by gravity loading, while the second technique uses deformations induced by the end-effector.

Chapter 14 describes and evaluates a method to track surface deformations using computer vision techniques, while Chapter 15 describes and evaluates a method to estimate deformations by finite-element model simulations using the SOFA framework.

The MURAB project is set up by a consortium of seven European partners: University of Twente (coordinator), University of Verona, RadboudUMC, KUKA, ZGT, MUW and Siemens. This project has received funding from the European Union's Horizon 2020 research and innovation programme under grant agreement No 688188 . 



\section{Part II}

\section{Pneumatic stepper motors}



CHAPTER 2

\section{Laser-CUtTing Pneumatics}

V. Groenhuis and S. Stramigioli. Laser-cutting pneumatics. IEEE/ASME Transactions on Mechatronics, 21(3):1604-1611, 2016. doi: 10.1109/TMECH. 2015.2508100 


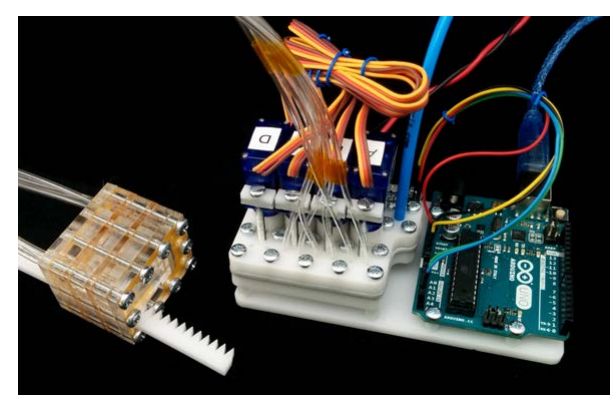

(a) Pneumatic linear stepper motor (left) and servo-controlled valve manifold (right).

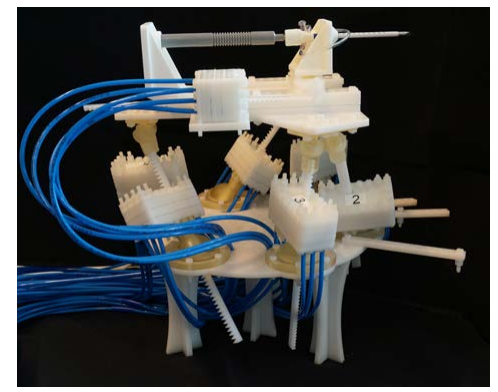

(b) MRI-compatible biopsy robot driven by pneumatic linear stepper motors.

Figure 2.1: Pneumatic devices.

\section{$2.1 \quad$ Abstract}

Pneumatic devices require tight tolerances to keep them leak-free. Specialized companies offer various off-the-shelf devices, and while these work well for many applications, there are also situations where custom design and production of pneumatic parts is desired. Cost efficiency, design flexibility, rapid prototyping and MRI compatibility requirements are reasons why we investigated a method to design and produce different pneumatic devices using a laser cutter from acrylic, acetal and rubber-like materials.

The properties of the developed valves, pneumatic cylinders and stepper motors were investigated. At 4 bar working pressure, the $4 / 3$-way valves are capable of $5 \mathrm{~Hz}$ switching frequency and provide at most $22 \ell / \mathrm{min}$ airflow. The pneumatic cylinder delivers $48 \mathrm{~N}$ of force, the acrylic stepper motor $30 \mathrm{~N}$. The maximum switching frequency over 6 metre long transmission lines is $4.5 \mathrm{~Hz}$, using $2 \mathrm{~mm}$ tubing. A MRI-compatible robotic biopsy system driven by pneumatic stepper motors is also demonstrated.

We have shown that it is possible to construct pneumatic devices using laser-cutting techniques. This way, plastic MRI-compatible cylinders, stepper motors and valves can be developed. Provided that a laser-cutting machine is available, the described pneumatic devices can be fabricated within hours at relatively low cost, making it suitable for rapid prototyping applications.

\subsection{Introduction}

Pneumatic cylinders are used in many applications. These come in different sizes and are being produced by many companies worldwide. The key elements are the bore, piston and seal, and are normally cylindrically shaped and made 


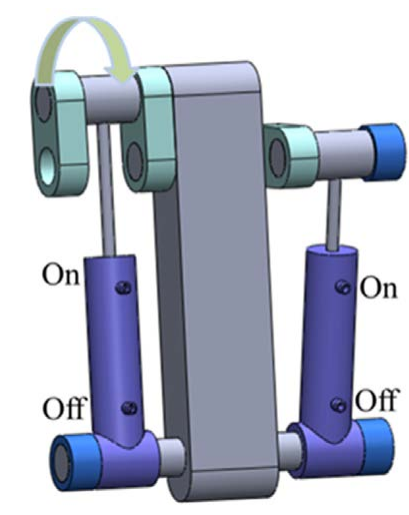

(a) Four-phase pneumatic motor (b) (Chen et al.)

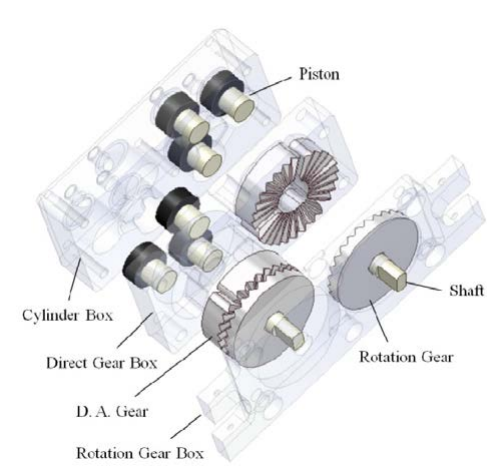

(Sajima et al.)

Figure 2.2: Two plastic pneumatic rotational stepper motors found in literature.

of metal. Pressurized air exerts a force on the piston, which causes it to slide within the bore. The sliding seal ensures that no air escapes from the chamber.

Sometimes, a custom cylinder design is desired, for example when integrating one or more cylinders in a small mechanical device. Also, MRI compatible systems restrict the usage of metallic materials, because of the strong magnetic field involved in MRI scanners. Furthermore, commercial pneumatic devices are often too expensive for low-cost projects by hobbyists. So there is a desire for a method to design and produce custom pneumatic parts quickly and at relatively low cost.

With the advent of accessible rapid prototyping services, more and more robotic devices are (partially) being 3d-printed [22, 72, 79, 86] or laser-cut [59], both by researchers and hobbyists. A laser cutter can cut out complex twodimensional shapes with high precision from plates of various materials. In this paper, we propose a method to assemble functional pneumatic devices (cylinders, valves and linear stepper motors) from laser-cut parts. The properties of these devices are then measured and discussed. A functional prototype of a MRI-compatible robotic device is also presented.

\subsubsection{Earlier research}

No earlier records involving functional laser-cut pneumatic devices could be found. While laser-cutting techniques are used extensively in different fields of engineering [59], it is (apparently) not yet used for manufacturing pneumatic devices. So, in this section we focus on existing MRI-compatible pneumatic (stepper) motor designs, as the MRI compatibility requirement is one of the 


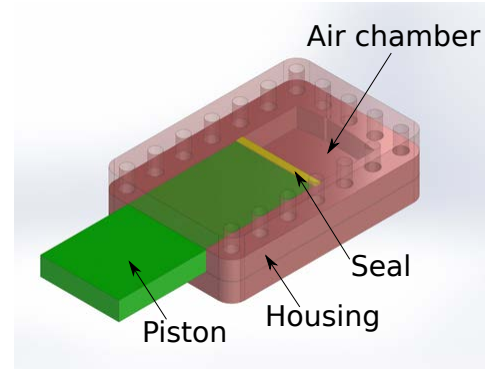

(a) CAD model

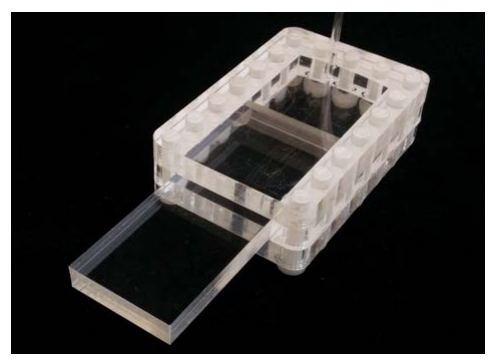

(b) Realization

Figure 2.3: Design and realization of a single-acting cylinder.

reasons to justify development of laser-cut pneumatic devices.

The PneuStep was developed in 2007 by Stoianovici et al. [118]. It is a rotational stepper motor with three chambers which are alternatingly pressurized, driving a circular gear. A different design of the same kind of motor is given in Figure 2.2b, which was developed by Sajima et al. [109].

Most off-the-shelf pneumatic cylinders involve metallic materials, but there also exist commercial plastic pneumatic cylinders. The miniature LEGO pneumatic cylinder (part $\mathrm{x} 189 \mathrm{c} 01$ ) is a fully plastic pneumatic cylinder which could be used in MRI-compatible systems. Chen et al. combined two of such cylinders to construct a four-phase rotational stepper motor (Figure 2.2a) [26]. While it proved to be effective, the motor is also quite large compared to the pneumatic cylinder size.

\subsection{Methods}

In this section, it is described how a laser-cut pneumatic piston can be designed and constructed.

\subsubsection{Cylinder geometry}

The basic cylinder consists of a housing assembled from multiple laser-cut parts, stacked and fixed together with screws. See Figure 2.3a for a CAD model. The housing basically consists of three layers (bottom, middle and top). Additional thin sheets can be used to increase the thickness of the middle layer. A piston is then placed in the opening of the middle layer, and a box-shaped rubber seal, adjacent to the piston, seals off the air chamber. When the parts are sufficiently smooth and well tightened together, then no gaskets are needed to avoid air leakages. This way, a single-acting pneumatic cylinder with an approximate rectangular cross-section is constructed. 


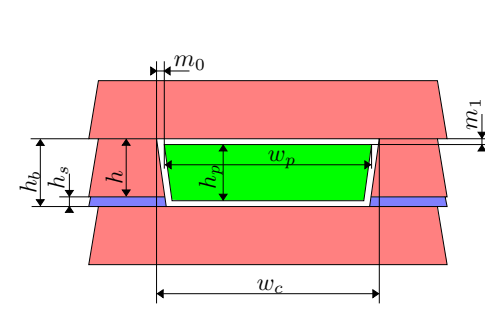

(a) Cylinder cross section (not to (b) Top view of cylinder scale) consisting of housing (red), pis- showing housing (red), ton (green) and spacer (blue).

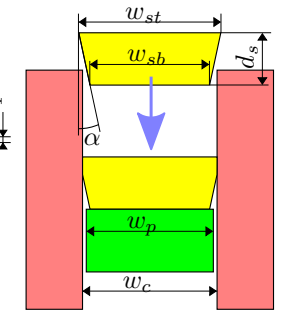

piston (green) and seal (yellow).

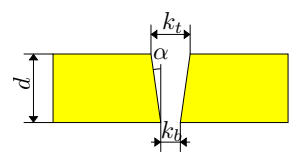

(c) Laser kerf dimensions.

Figure 2.4: Cylinder and kerf geometries.

\subsubsection{Materials}

Poly(methyl methacrylate) (PMMA, acrylic, Plexiglas §, Perspex@, from now on called 'acrylic') and Polyoxymethylene (POM, acetal, Delrin $₫$, Ertacetal@, from now on called 'acetal') are smooth, strong plastics that are well suitable for laser-cutting. These can be used for the cylinder housing and the piston. Extruded acrylic plates tend to have less variations in thickness than cast acrylic. Acetal plates also tend to be more constant in thickness than acrylic plates.

Sheets of paper or polyester $(0.1-0.2 \mathrm{~mm})$ can be used as spacers. Silicone rubber or Trotec Laserrubber (a rubber-like material intended for laserengraving stamps) of thickness 1.5-3mm can be used for sliding seals within the bore, and for pneumatic routing between plates. Standard off-the-shelf pneumatic tubing (e.g. polyurethane, $2 \mathrm{~mm}$ or $4 \mathrm{~mm}$ ) is used to supply air to the chambers.

Metal (brass, steel) or plastic (nylon) screws can be used as fastener, in combination with nuts or tapped holes in the bottom (or top) part. Metal screws can yield higher compression forces, but only the plastic ones are MRI compatible. A sealant such as blue silicone (Loctite@5926) can also be used to make the housing completely airtight.

\subsubsection{Dimensions and tolerances}

Pneumatic cylinders only work smoothly and leak-free when the dimensions of all parts are accurately designed and fabricated. See Figure 2.4a for a crosssection of the cylinder, showing the housing (red), piston (green) and spacer(s) (blue). (For interpretation of the references to colors in the figure, the reader is referred to the web version of this paper.)

The cylinder housing needs to have relatively thick walls, to resist bulging of the parts under pressure. This is a limitation that circular cylinders do not 
have.

To slide a piston smoothly and without wobbling, there should be some small clearance around all sides $\left(m_{0}\right.$ and $m_{1}$ in Figure $\left.2.4 \mathrm{a}\right)$, in the order of $0.05-0.10 \mathrm{~mm}$. So we need to have an air chamber height of $h_{b}=h_{p}+$ $0.15( \pm 0.05) \mathrm{mm}$. One option is to use a spacer with thickness $h_{s}=0.15( \pm 0.05) \mathrm{mm}$. Another option is to reduce the piston plate's thickness $h_{p}$ using laser engraving, or by grinding with sand paper. A third option is to cut the parts out of different plates (or from different regions of the same plate), from which the difference in plate thickness $h-h_{p}=0.15( \pm 0.05) \mathrm{mm}$.

The seal is constructed by cutting out a rectangular (or trapezoidal) shape from a sheet of rubber-like material. Many other seal types (e.g. lip seals) cannot be easily manufactured by laser-cutting, and off-the-shelf seals are not available for rectangular cylinders.

See Figure 2.4b for a top view of the seal's geometry. The seal (yellow) must fully cover the cross-sectional area of the bore to avoid leakage of air: $w_{s t}>w_{c}$. It is also required to have $w_{s b}<=w_{p}$ to avoid jamming of the seal between the piston (green) and cylinder housing (red). So the seal needs to have a trapezoidal cross-section. When the seal is laser-cut, the laser kerf' edges are slanted with some angle $\alpha$ which can be exploited to obtain the desired shape. It is also possible to hand-cut the seals with a knife; while this is less accurate than laser-cutting, it allows for a larger angle $\alpha$ and thus more tolerance. See the right part of Figure 2.5, for some laser-cut and hand-cut seals, photographed from different sides.

Because of the thickness variation of plates, the dimensioning of certain parts (e.g. seals) may need to be adapted to the actual thickness of other parts (e.g. $h_{b}$ in Figure 2.4a). The manufacturing procedure is as follows: 1. Manufacture cylinder housing and piston. 2. Measure $h_{b}$. 3. Design and manufacture seal. 4. Evaluate performance of assembled cylinder. 5. In case of air leakage or excessive seal friction, repeat from step 3.

\subsubsection{Kerf geometry}

When the laser cutter cuts out a piece, material is molten and evaporated along the cutting line. The gap is called the kerf (see Figure 2.4c), and knowledge about its geometry is essential to obtain parts with the right dimensions. Its cross-sectional shape is approximately trapezoid. The dimensions $\left\{k_{t}, k_{b}\right\}$ depend on the material type, thickness $d$, laser type, lens' focal distance and focal point, cutting power, speed, frequency, assistant gas and the local temperature which in turn depends on the cutting trajectory. The kerf's edges should be as smooth as possible, as grooved edges negatively affect cylinder performance. The optimal settings to obtain a good, clean cut can be determined experimentally. When the working settings are determined for a certain material, its kerf can be measured and be accounted for in the initial design, and then be 


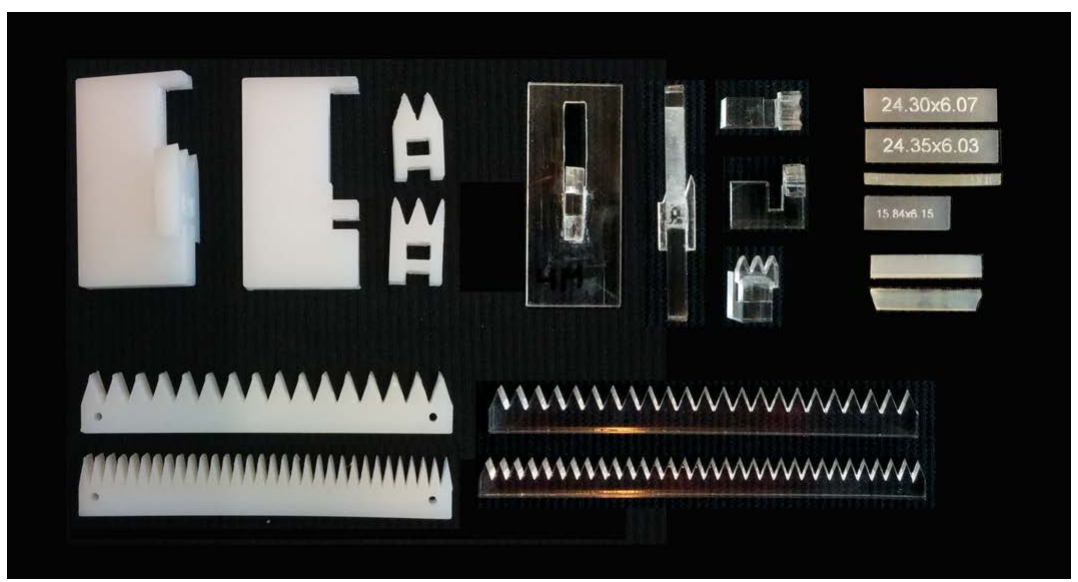

Figure 2.5: Varous parts laser-cut from acetal (left), acrylic (middle) and silicone rubber (right).

further optimized experimentally.

The trapezoidal shape of the kerf implies that all walls of the cut-out parts have slanted edges with angle $\alpha$. This is not necessarily a problem, as it can be accounted for in the design. For example, the piston can be placed upsidedown in the housing (visualized in Figure 2.4a) so that the slanted edges of the housing and piston are approximately parallel. The seals also make use of the slanted edges resulting from laser-cutting, to control the difference in dimensions $w_{s t}$ and $w_{s b}$ in Figure $2.4 \mathrm{~b}$.

\subsection{Production}

\subsubsection{Cylinders}

In this section, the production process of several pneumatic cylinders and other parts are described, in increasing complexity.

\section{Single-acting cylinder}

The simplest design is a single-acting cylinder. It consists of just one chamber which can be pressurized, pushing away a box-shaped piston. The design is given in Figure 2.3a, and the realization in Figure 2.3b. The housing $(40.0 \mathrm{~mm}$ x $60.0 \mathrm{~mm}$ x $17.5 \mathrm{~mm}$ ) and piston parts were cut out of $6 \mathrm{~mm}$ extruded acrylic (actual thickness $(5.77 \pm 0.03) \mathrm{mm}), 0.1 \mathrm{~mm}$ polyester foil was used as spacer, and $2 \mathrm{~mm}$ thick silicone rubber as seal. Nylon M4 screws were used to hold the housing together. The bore dimensions are $w=24.0 \mathrm{~mm}, h=5.87 \mathrm{~mm}$, giving a 


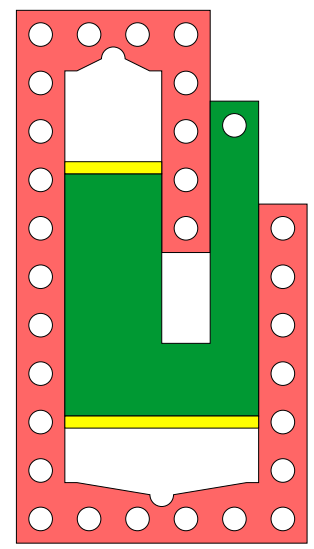

(a) Top view of design

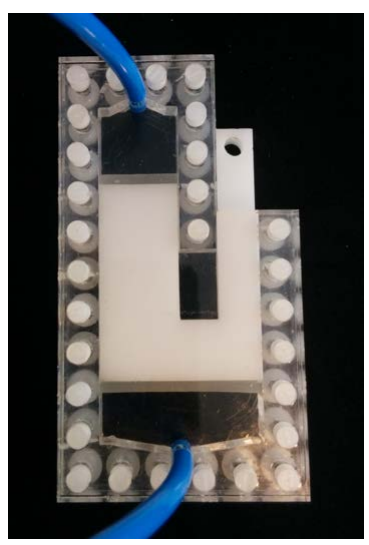

(b) Realization

Figure 2.6: Design and realization of double-acting cylinder.

cross-sectional area of $141 \mathrm{~mm}^{2}$. The theoretical force exerted by the piston is then $F=P \cdot A=141 \cdot 10^{-6} P$ (equivalent to $84.6 \mathrm{~N}$ at 6 bar).

The seal is trapezoidally shaped, and different dimensions were tested. Eventually, the optimal shape was found to be a trapezoid sized $24.44 \mathrm{~mm}$ x $6.01 \mathrm{~mm}$, with slanted edges of $1.0^{\circ}$.

The piston can extend all way out of the cylinder (travel $50 \mathrm{~mm}$ ), and there is no return mechanism. Also, because the seal is not affixed to the piston but sliding freely, outstroke movements are only allowed when the cylinder chamber is pressurized. Otherwise, the seal would lose contact with the piston, and become dislocated rendering it ineffective. In practice, it depends on the application whether this is a problem or not. It is also possible to implement an (elastic) spring return mechanism, but an important drawback is that this considerably reduces the effective force of the pneumatic cylinder.

\section{Double-acting cylinder}

A double-acting cylinder is more useful than a single-acting cylinder, as this can perform both outstroke and instroke motions. The simplest way is to connect to single-acting cylinders opposite to each other. One design is shown in Figure 2.6a. It consists of a J-shaped piston (acetal, green) with a protruding rod, in an acrylic housing ( $88 \mathrm{~mm}$ length). There are two chambers which are sealed off with silicone rubber seals (yellow) and act on the piston. Depending on which chamber is pressurized, outstroke or instroke motion (travel $24 \mathrm{~mm}$ ) is performed. The rod does not pass through either chamber, because it would be very difficult to seal it properly. 


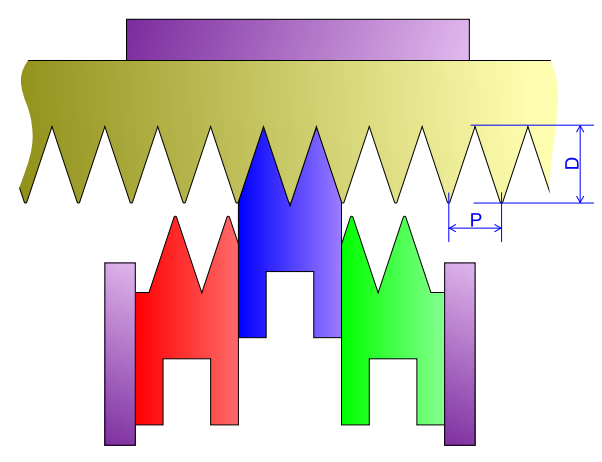

Figure 2.7: Stepper motor mechanism showing rack (yellow) and three pistons (red, blue, green).

\subsubsection{Stepper motors}

A double-acting cylinder has two well-defined states, with the piston being in either extreme position. Controlling the piston to intermediate positions as well is difficult: it would require position feedback and precise pressure control, and it would result in a compliant actuator due to the compressibility of air which is generally not desired. So in this section a different mechanism, a three-phase pneumatic linear stepper motor, is presented which is relatively easy to drive and allows discrete position control of an arbitrary long rack.

The schematic mechanism of the stepper motor is shown in Figure 2.7. The rack (yellow) slides to left or right, when the three toothed pistons (red, blue, green) move up and down in the correct order, working as a wedge on the rack. The step size is one-third of the pitch $P$. Each piston is driven by a separate double-acting cylinder, so there are six pressure chambers in total.

\section{Symmetric stepper motor}

The symmetric stepper motors (Figure 2.8a, right, and Figure 2.8b) use rectangular pistons. First, the housing was constructed, consisting of seven layers sized $72 \times 36 \mathrm{~mm}$ from a $4 \mathrm{~mm}$ thick acrylic or acetal plate. A few spacers $(0.1$ or $0.2 \mathrm{~mm}$ paper or polyester) were also cut out, and also the $4 \mathrm{~mm}$ thick pistons (without teeth). The spacers were added between the layers such that the pistons could just slide smoothly with as little clearance as possible, while the housing was tightened with the (metallic or nylon) screws. The resulting bore cross-sectional area is $20.0 \mathrm{x} 4.10 \mathrm{~mm}^{2}=82.0 \mathrm{~mm}^{2}$.

After finishing the housing, the seals were produced. In certain motors the seals were cut with a knife, in the other ones the seals were laser-cut. The seal's dimensions were repeatedly adjusted until the pneumatic cylinders 


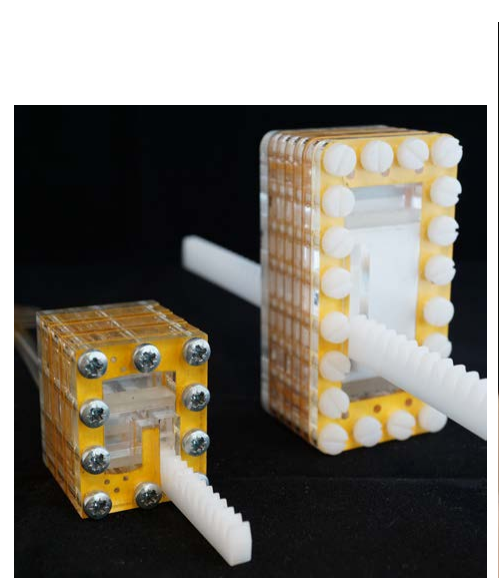

(a) Small (left) and large (right) acrylic/acetal motors

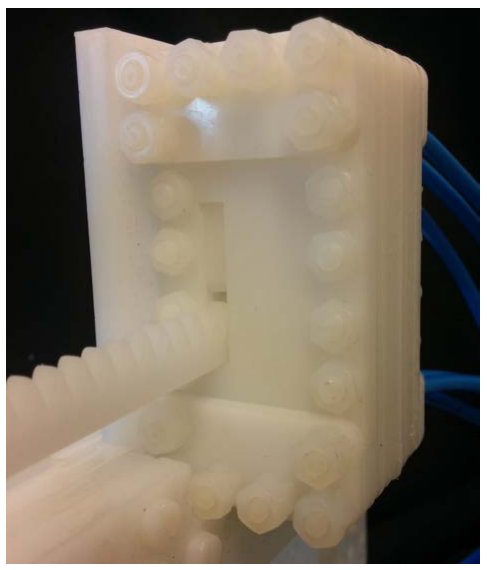

(b) Fully acetal motor

Figure 2.8: Various stepper motors made of different combinations of acrylic and acetal.

operated smoothly with neither significant air leakage nor excessive friction.

Next, the exact distances between the pistons in the housing were measured in order to calculate the piston teeth shape, as these have to be phased $120^{\circ}$ apart. After laser-cutting the teeth pieces (pitch 2, 3 or $4 \mathrm{~mm}$, and depth 5 or $6 \mathrm{~mm}$ ), the acrylic ones were glued to the pistons, while the acetal teeth were snap-fit into its locations. Figure 2.5 shows some pistons and racks made from both materials.

\section{Miniature asymmetric stepper motor}

The available volume within the symmetric stepper motor can be used more efficiently by using the asymmetric double-acting cylinders as described in Section 2.4.1. As the piston only performs effective work during outstroke, less force is needed for the instroke motion which can be driven by a smaller chamber.

The layer thickness was also optimized to achieve a cross-sectional area of $15.50 \times 6.10 \mathrm{~mm}=94.6 \mathrm{~mm}^{2}$ for the outstroke, which is slightly higher than that of the symmetric stepper motor. The resulting miniature motor (Figure 2.8a, left) measures $36 \times 28 \times 30 \mathrm{~mm}$, a $58 \%$ size reduction compared to the symmetric stepper motor sized $72 \times 36 \times 28 \mathrm{~mm}$. 


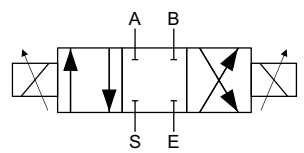

(a) Left: 4/3-way servo valve symbol. Right: Corresponding positions of valve wheel.
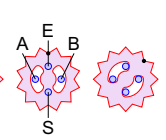
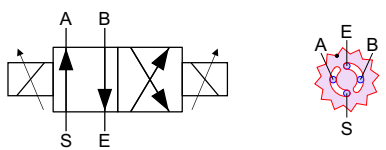

(b) 4/2-way servo valve symbol (left) and wheel positions (right).

Figure 2.9: Schematics and working principle of 4/3-way and 4/2-way servo valves. Pairs of orifices (blue) are connected depending on the valve wheel position (red). S: pressurized air supply; E: exhaust; A and B: outputs to double-acting pneumatic cylinder.

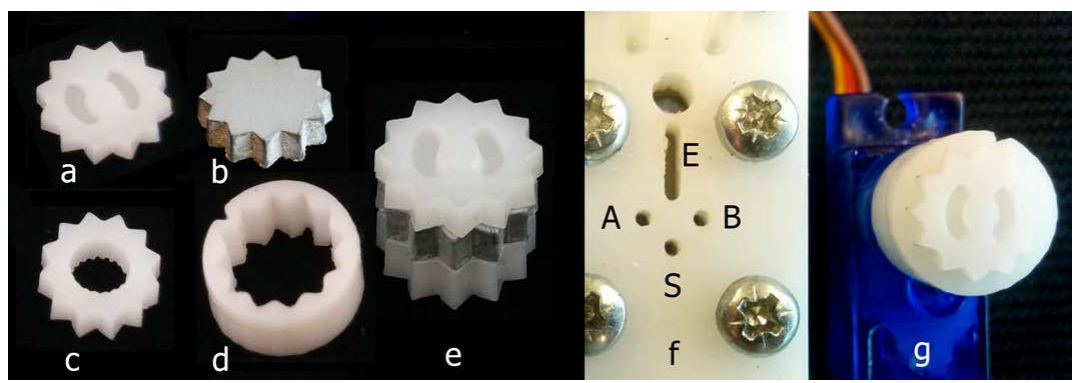

Figure 2.10: Valve wheel (a), elastomeric spring (b), servo shaft connector (c), spindle (d), stack of parts a-c (e), base plate with orifices and exhaust (f), gear shaft coupler (parts a-d) mounted on servo $(\mathrm{g})$.

\subsection{Valves}

Valves are needed to control the pneumatic cylinders and stepper motors described in the previous sections. A valve consists of a mechanism that can open and close pneumatic connections. Like the pneumatic cylinders, the valves can also be constructed with laser-cutting techniques. In this section, several design strategies and motorized pneumatic valves are presented.

\subsubsection{Servo valve}

A piece of $2 \mathrm{~mm}$ thick acetal, laser-cut (9mm outer diameter) and engraved to produce grooves (1mm depth) based on the design in Figure 2.9a (right), shown in Figure 2.10a, is pressed against an acetal plate with $1 \mathrm{~mm}$ orifices (Figure 2.10f). The exhaust orifice is a $1 \mathrm{~mm}$ groove. By rotating the wheel over $45^{\circ}$, the interconnections between the orifices are changed and different functional states (Figure 2.9a) can be reached. Variable flow control is also possible by micro-adjusting the wheel position using an $\mathrm{r} / \mathrm{c}$ servo. The wheel is connected to this servo by means of a gear shaft coupler with an elastomeric spring (Figure 2.10e), to compensate for any angular misalignment. Thanks to 


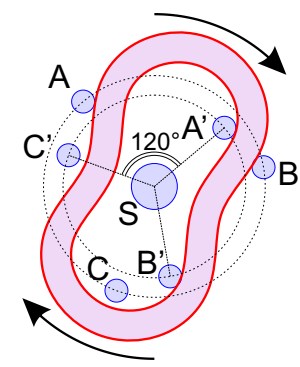

(a) Schematic layout.

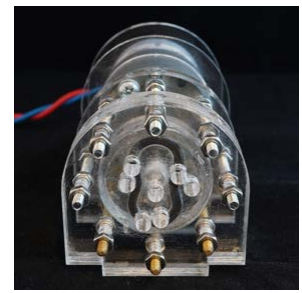

(b) Front view

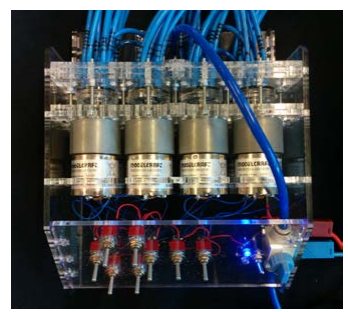

(c) Top view of eight-fold manifold

Figure 2.11: 8/6-way pneumatic distributor, design and implementations. The diagram on the left shows the rotating rubber ring (red) and orifices (blue). S: pressurized air supply; A, B, C, A', B', C': outputs to stepper motor. Dimensions not to scale.

the low inherent friction of acetal, relatively little torque is needed to rotate the part which can be performed by a low-cost micro servo like the Hextronic HXT900 or Modelcraft YH-3009 as used here.

The specified switching time of a common micro servo is $0.08 \mathrm{~s}$ per $50^{\circ}$. For a $4 / 3$-way valve, a rotation of $90^{\circ}$, taking $0.14 \mathrm{~s}$, is needed to toggle a pneumatic cylinder. Some additional time is needed for acceleration and to change the pressure inside the tubing and chambers, before the piston can start moving to its new position. The upper limit of the operating frequency is thus below $7.0 \mathrm{~Hz}$. For a $4 / 2$-way valve, the switching angle is $45^{\circ}$ which can be performed in approximately half the time.

Multiple valves can be placed in a row, to form a manifold. In Figure 2.1a (right), four valves are mounted adjacently in one package.

\subsubsection{Motor-controlled 8/6-way pneumatic distributor}

A pneumatic stepper motor as decribed in Section 2.4 .2 contains three doubleacting pistons. These could be controlled with three independent $4 / 2$-way valves, but it is also possible to control all three pistons with a single motordriven 8/6-way pneumatic distributor. The continuously rotating part houses an 8-shaped rubber ring, which is tightly pressed against an acrylic plate with seven orifices.

See Figure 2.11a for the ring and orifices layout. Pressurized air enters the center orifice $(\mathrm{S})$, and flows through those other orifices that are in the interior area of the rubber ring. The orifices that lie outside the rubber ring's perimeter are allowed to exhaust air to the environment directly. The ring is point-symmetric, so that the forces are balanced and the ring does not tilt to one side.

In one full cycle in which the rubber ring rotates $180^{\circ}$, each of the six orifices 
are pressurized and de-pressurized at different moments within the cycle. The duty cycle (ratio of pressurization time) depends on the distance from the rotational center, while the phase within the cycle depends on the angular position. With the configuration in Figure 2.11a, the six orifices can be connected to the six chambers of a stepper motor in such a way that each piston goes up and down once per cycle, phased $120^{\circ}$ apart; during $33 \%$ of the time two pistons are pushing against the rack and during $67 \%$ of the time only one piston pushes against the rack. This is meant to reduce unnecessary stress on the teeth. The speed can be controlled by changing motor current, and some form of position feedback (e.g. with an optical quadrature encoder) would be required to track the revolutions. Realizations of the design are shown in Figure 2.11b and Figure 2.11c.

\subsection{Applications in a MRI-compatible robotic system}

A 7 DOF MRI-compatible biopsy robot has been designed and built (Figure $2.1 \mathrm{~b})$, using the symmetric acetal stepper motors as actuators. The kinematic design consists of a 6 DOF hexapod (also known as a Stewart platform), plus a single DOF needle insertion mechanism on top of the platform as the endeffector. The ball joints are all 3d-printed by the Stratasys Objet Eden250 printer with FullCure720 material, and the other structural parts are mostly laser-cut from acetal. Except for the needle itself, all parts of the biopsy robot are made of plastic and thus (theoretically) MRI-compatible.

The robot is driven by a pneumatic distributor system as shown in Figure 2.11c. It consists of eight motorized 8/6-way valves as described in Section 2.5.2, and are manually operated.

\subsection{Measurements}

The maximum switching frequency of pneumatic cylinders and stepper motors should be sufficiently high for the application. This mainly depends on the working pressure, valve switching speed and airflow, tube dimensions and pneumatic cylinder displacement volume. For pneumatic cylinders and stepper motors, the net force is an important characteristic. To learn about these parameters, various measurements were performed which are described in this section. 


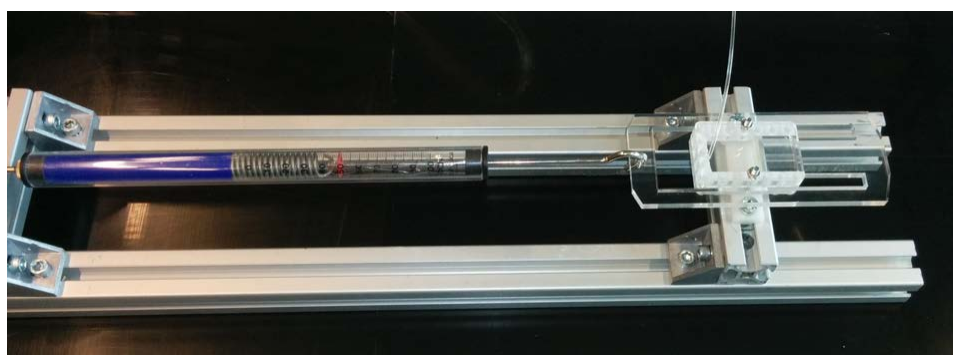

Figure 2.12: Test setup for force measurements of cylinders and stepper motors.

\subsubsection{Setup}

The actuation force of pneumatic cylinders and stepper motors were measured with the test rig as shown in Figure 2.12. An acrylic single-acting cylinder with a cross-sectional bore area of $141 \mathrm{~mm}^{2}$ was put under increasing and then decreasing pressure from 0.5 to 6 bar, while the load on the rod was measured with a spring scale. Three different seals were included in the test.

An acrylic stepper motor with cross-sectional bore area of $78.4 \mathrm{~mm}^{2}$ was also tested with the same setup. The teeth depth was $6 \mathrm{~mm}$ and the pitch was $2 \mathrm{~mm}$. The pressure varied from 1 to 4 bar (gauge pressure); for each pressure level the stepper motor was operated very slowly, up to the point that the rack could no longer overcome the spring scale force. The test was then repeated with a different rack and set of pistons, now with a pitch size of $4 \mathrm{~mm}$.

The valve airflow was measured by filling a 1.35 litre tank, while the transient pressure was being recorded. The working pressure varied from 1 to 6 bar. The maximum airflow at each working pressure level was derived from the highest rate of pressure change, and also compared with the airflow resulting from de-pressurizing the air tank.

The maximum switching frequency of a pneumatic valve/cylinder pair, connected with tubes of different widths $(2 \mathrm{~mm}, 4 \mathrm{~mm}$ and $6.3 \mathrm{~mm}$ outer diameter, having $1.4 \mathrm{~mm}, 2.5 \mathrm{~mm}$ and $4.3 \mathrm{~mm}$ inner diameter respectively) and different lengths $(0.15 \mathrm{~m}, 2 \mathrm{~m}, 4 \mathrm{~m}$ and $6 \mathrm{~m})$, was evaluated by increasing the switching frequency while monitoring the behaviour of a double-acting cylinder with displacement volume of $0.51 \mathrm{ml}$. The highest switching frequency at which the piston still consistently moved to either extreme position, was recorded for each tube dimension, at different pressure levels (1 to 6 bar). 


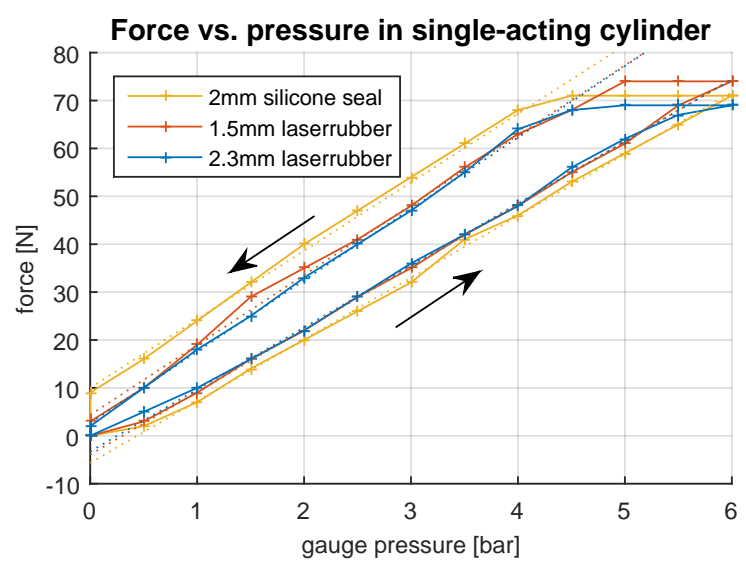

Figure 2.13: Force measurements on single-acting cylinder.

\subsubsection{Results and discussion}

\section{Single-acting cylinder force measurements}

Figure 2.13 shows the force as function of applied pressure for the single-acting cylinder, for three different seals. There is some hysteresis caused by the friction of the seal, so the measured force during increasing pressure (upwards leg) is lower than during decreasing pressure (downwards leg).

We observe that there is a good linear fit for each leg. The average slope $\Delta F / \Delta P$ on the upwards leg is $13.0 \mathrm{~N} /$ bar or $130 \cdot 10^{-6} \mathrm{~N} / \mathrm{Pa}$, and the average slope for the downwards leg is $14.6 \mathrm{~N} /$ bar or $146 \cdot 10^{-6} \mathrm{~N} / \mathrm{Pa}$. This is in good agreement with the theory: $\Delta F / \Delta P=141 \cdot 10^{-6} \mathrm{~N} / \mathrm{Pa}$.

The offset force, which can be fully attributed to the seal friction, is approximately $3 \mathrm{~N}$ for the $2 \mathrm{~mm}$ silicone seal, $4 \mathrm{~N}$ for the $1.5 \mathrm{~mm}$ Trotec Laserrubber seal and $8 \mathrm{~N}$ for the $2.3 \mathrm{~mm}$ Trotec Laserrubber seal.

\section{Acrylic stepper motor force measurements}

Figure 2.14 shows the force measurements of the acrylic stepper motor. For a pitch size of $4 \mathrm{~mm}$, all measurement data fit on a straight line, crossing the horizontal axis at 0.09 bar and having a slope of $8.1 \mathrm{~N} /$ bar or $81 \cdot 10^{-6} \mathrm{~N} / \mathrm{Pa}$. Again, the horizontal offset $(0.09$ bar $)$ can be attributed to the friction of the silicone piston seal. The maximum force was found to be $24 \mathrm{~N}$ at 4.0 bar pressure.

The theoretical piston force, calculated from the bore cross-sectional area, is $F=78.4 \cdot 10^{-6} \mathrm{~N} / \mathrm{Pa}$. The mechanical advantage in the wedge is $\alpha=\frac{2 D}{P}$ (Figure 2.7). For depth $D=6 \mathrm{~mm}$ and pitch $P=4 \mathrm{~mm}$, this ratio is $\frac{2 \cdot 6}{4}=3$, so the theoretical rack force is $F=3 \cdot 78.4 \cdot 10^{-6} \mathrm{~N} / \mathrm{Pa}=235 \mathrm{~N} / \mathrm{Pa}$. 


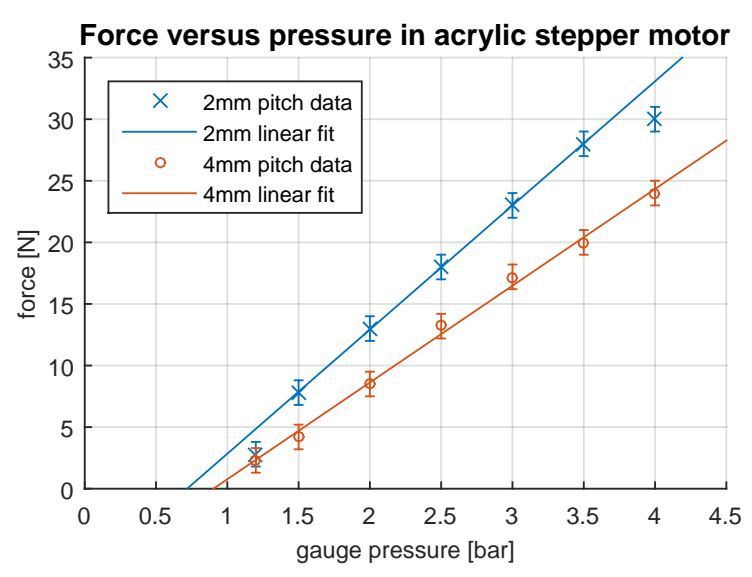

Figure 2.14: Force measurements on acrylic stepper motor, with two different pitch sizes.

The efficiency of the piston-rack transfer can now be found to be $\eta=\frac{81}{235}=$ $34 \%$, so $66 \%$ of the force is lost due to friction for this particular type of motor.

When a smaller pitch size is used ( $2 \mathrm{~mm}$ instead of $4 \mathrm{~mm})$, then the force becomes higher. The maximum force was now found to be $30 \mathrm{~N}$ at a pressure of $4.0 \mathrm{bar}$, and ignoring the two outliers, the force/pressure ratio was found to be $10 \mathrm{~N} /$ bar and the efficiency $\eta=\frac{100}{470}=21 \%$.

\section{Valve airflow measurements}

Table 2.1: Valve airflow at different working pressure levels

\begin{tabular}{c|c} 
pressure [bar] & max. airflow $[\ell / \mathrm{min}]$ \\
\hline 1.0 & 8.0 \\
2.0 & 13 \\
3.0 & 18 \\
4.0 & 22 \\
5.0 & 27 \\
6.0 & 32
\end{tabular}

The airflow was measured for different working pressures and the results are given in Table 2.1. The given airflow is the highest measured airflow while pressurizing a 1.35 litre air tank. The airflow is mainly restricted by the diameter of the orifices in the valve, which measure approximately $1 \mathrm{~mm}$ in diameter The effective orifice diameter is about $0.8 \mathrm{~mm}$. The depressurization airflow was also measured and found to be approximately equal or slightly higher than the pressurization airflow. 


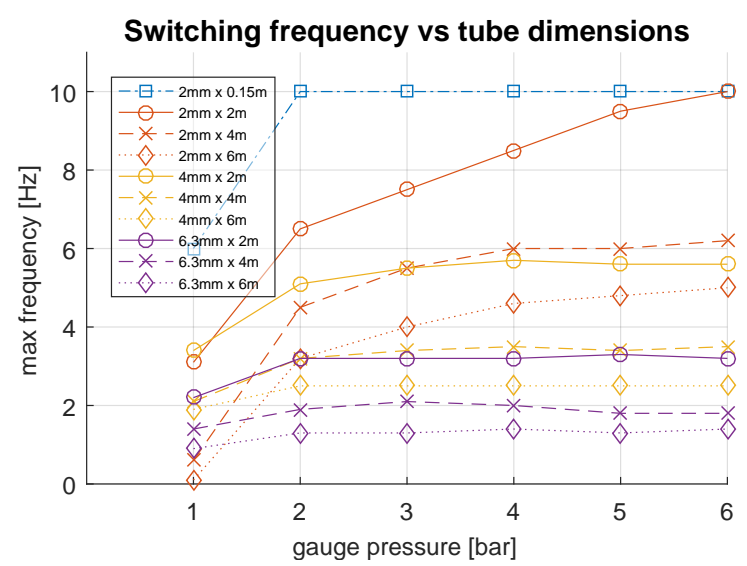

Figure 2.15: Maximum switching frequency of a double-acting cylinder for various pressure levels and tube length/diameter sizes.

\section{Switching frequency measurements}

See Figure 2.15 for the measurement results involving driving a double-acting pneumatic cylinder through tubes of different sizes, using a $4 / 2$-way valve, at different working pressure levels.

We can make the following observations:

- Highest switching speeds are achieved using short and thin tubes. The maximum valve switching speed $(10 \mathrm{~Hz})$ is achieved when using the $2 \mathrm{~mm}$ $\mathrm{x} 0.15 \mathrm{~m}$ tube at least 2 bar working pressure, or the $2 \mathrm{~mm} \times 2 \mathrm{~m}$ tube at 6 bar working pressure.

- At 1 bar working pressure, the piston only moves slowly, and the switching speed is relatively low for all tube dimensions.

- Increasing pressure beyond 2 bar has little effect on $4 \mathrm{~mm}$ or $6.3 \mathrm{~mm}$ thick tubes. The maximum valve airflow in combination with the tube dimensions seems to be the limiting factor here. For example, a $6.3 \mathrm{~mm} \times 6 \mathrm{~m}$ tube has a volume of $87 \mathrm{ml}$. To toggle the cylinder, the pressure difference across the chambers must be at least 1 bar. At 2 bar working pressure, it takes at least $1.5 \frac{87}{217} \mathrm{~s}=0.60 \mathrm{~s}$ to increase the pressure from zero to 1.5 bar. At 6 bar working pressure, it takes at least $3.5 \frac{87}{537} \mathrm{~s}=0.57 \mathrm{~s}$ to increase the pressure from zero to 3.5 bar. Both figures correspond well with the measured switching time of $0.75 \mathrm{~s}$ or $1.3 \mathrm{~Hz}$.

- For $2 \mathrm{~mm}$ thin tubes, the resistance in the tubes seems to play a significant role as well because the measured switching time is higher than the time required to pressurize the tube. 
- For $6 \mathrm{~m}$ long tubes, the best thickness is $2 \mathrm{~mm}$ and the maximum switching frequency is $4.5 \mathrm{~Hz}$. Only when the valve airflow is significantly increased, it would make sense to use thicker tubes.

\subsection{Conclusion}

We have described methods to systematically design and produce valves, pneumatic cylinders and stepper motors using laser-cutting techniques. The problem of varying plate thickness has been circumvented and the described methods to make chambers airtight using screws and seals turn out to work effectively.

The $4 / 3$-way valve is capable of $5 \mathrm{~Hz}$ switching speeds (or $10 \mathrm{~Hz}$ for the $4 / 2$-way valve). The valve allows working pressures up to 6 bar, and has a maximum airflow of $22 \ell / \mathrm{min}$ at 4 bar due to the $1 \mathrm{~mm}$ orifices in the valve.

The tested single-acting pneumatic piston can exert forces up to about $70 \mathrm{~N}$ (at 6 bar), and the tested stepper motor exerts up to $30 \mathrm{~N}$ of force (at 4 bar and $2 \mathrm{~mm}$ pitch) with $22 \%$ efficiency.

When 6 metre long pneumatic tubes are required, e.g. when driving a MRI-compatible pneumatic robotic system with valves placed outside the MRI room, then the tubes should be $2 \mathrm{~mm}$ in diameter and the maximum switching frequency is then $4.5 \mathrm{~Hz}$ (at 4 bar). To increase this frequency, the valve airflow should be increased, by enlarging the orifices in the valve.

For researchers and hobbyists that have access to a laser cutter, the described methods provide a novel way of designing and producing low-cost custom pneumatic devices. 
RaPid Prototyping High-Performance MR Safe Pneumatic Stepper Motors

V. Groenhuis and S. Stramigioli. Rapid Prototyping High-Performance MR Safe Pneumatic Stepper Motors. IEEE/ASME Transactions on Mechatronics, 23(4):1843-1853, 2018. doi: 10.1109/TMECH.2018.2840682 


\subsection{Abstract}

In this paper, we show that MR safe pneumatic stepper motors can be constructed using rapid prototyping techniques such as 3-D printing and lasercutting. The designs are lightweight, metal-free and fully customizable. Besides MRI-compatible robotic systems, other potential applications include highvoltage switchgear and nuclear power plant systems which also restrict electric actuation. In addition, it can be applied to other actuation systems where pressurized air is available and lightweight, rapid prototypeable actuators are needed.

Five pneumatically-driven linear and rotational stepper motors have been developed, with forces up to $330 \mathrm{~N}$, torques up to $3.7 \mathrm{~N} \mathrm{~m}$, stepping frequency up to $320 \mathrm{~Hz}$, dimensions ranging from $25 \mathrm{~mm}$ to $80 \mathrm{~mm}$, free of backlash and power up to $26 \mathrm{~W}$. All five motors are constructed from six 3-D printed parts and four seals, held together by nylon screws or clips. The described stepper motors outperform state-of-the-art plastic pneumatic stepper motor designs, both in specifications and in manufacturability.

\subsection{Introduction}

Rotational stepper motors are widely used in actuation of mechanical devices. Off-the-shelf stepper motors are generally driven by electromagnetic forces, constructed from an electromagnetic stator and a permanent magnet rotor. The stator has two or more phases, each consisting of an electromagnetic coil which can generate a magnetic field to apply a torque on the rotor. By driving the coils with appropiate waveforms, step-wise rotational motion is achieved. A rack-and-pinion or leadscrew mechanism can convert rotational to translational movements, but pure electromagnetic linear stepper motors also exist in which the stator is a track of magnets on which a moving platform with electromagnetic coils can slide back and forth.

One application for stepper motors is in medical robotics. In breast cancer screening and diagnosis, an MRI-guided breast biopsy may be required to acquire tissue samples for pathology assessment to determine if a lesion is malignant. The current manual MRI-guided breast biopsy procedure is inaccurate and would benefit from a robotically-driven needle positioning and insertion system that can operate inside the bore. Such a robot needs to be driven by MR safe/conditional actuators. Electromagnetic motors are not an option due to interference with the strong magnetic field, which would pose safety hazards and distort MRI scans. Taking the available space and the range of forces into account, we can specify the requirements for a linear actuator as follows: free of conductive materials, size under $50 \mathrm{~mm}$, positional accuracy $1 \mathrm{~mm}$ or better, force at least $50 \mathrm{~N}$ and velocity up to $5 \mathrm{~mm} / \mathrm{s}$. The motor must be controllable 


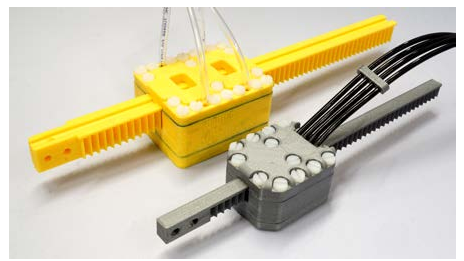

(a) Linear motors: T-63 (top) and T-49 (bottom).

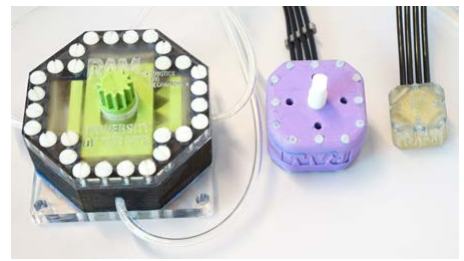

(b) Rotational motors: R-80 (left), R-44 (middle) and R-25 (right).

Figure 3.1: Pneumatic stepper motors.
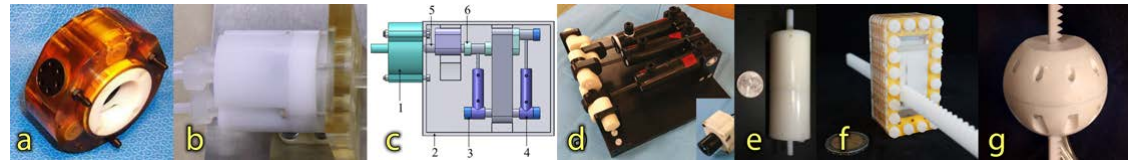

Figure 3.2: State-of-the-art pneumatic stepper motors: a. Stoianovici et al. (2007), b. Sajima et al. (2012), c. Chen et al. (2014), d. Secoli et al. (2015), e. Guo et al. (2016), f. Groenhuis and Stramigioli (2014), g. Groenhuis et al. (2015).

from outside the Faraday cage of the MRI scanner, i.e. over a distance of at least $5.0 \mathrm{~m}$, and the motor should be scalable and rapid prototypeable so that motors can be easily replicated and adapted for specific joints within particular kinematic configurations.

\subsubsection{State of the art}

Since electromagnetic motors are not MR safe or conditional, various alternative actuation methods have been investigated. While hydraulic $[42,133]$, piezo [93, 122], cable transmission [24], MRI-driven [40], air turbine [37], flexible fluidic actuators [30], direct-acting pneumatic actuators [135] and unidirectional pneumatic stepper motors [27] have been demonstrated, actuation by metalfree bidirectional pneumatic stepper motors has several important advantages. They are inherently MR safe, tolerant for small air leakages, can be controlled with a standard pneumatic valve manifold and allow for step-wise position control without need for a position feedback system.

Figure 3.2 shows, and Table 3.1 (a-g) lists a number of MR safe/conditional pneumatic stepper motors found in literature. They are compared by specifications such as motor dimensions, step size, force, stepping frequency and power. Because there is no uniform test protocol, not all figures are directly comparable. This especially applies to the maximum power, for which certain authors push the motor outside the normal operation range using short tubes, high pressure and fast valves, while other authors only perform measurements using a practical setup with longer tubes and/or slower valves. It might there- 
fore also be useful to compare the maximum work (force times displacement) performed in one single step, hereby ignoring the stepping frequency.

Stoianovici et al. developed the PneuStep (Figure 3.2a) [118]. This design avoids sliding parts as much as possible by using diaphragm sealing and ball bearings. It provides $3 \mathrm{~W}$ output power in normal operation range, and up to $37 \mathrm{~W}$ when pushed for power. However, the PneuStep design is relatively large and also very complex to manufacture due to the 26 different components made out of 11 materials. The design of Sajima et al. (Figure 3.2b) is much more compact and easy to manufacture, and still offers good properties considering its size [110]. The Lego-powered motor by Chen et al. (Figure 3.2c) performs much work per step and uses a gearbox to obtain high output torque, but it was only tested at low speeds (4 steps/sec) resulting in a rated power of $0.13 \mathrm{~W}$ which is relatively weak for its $\operatorname{size}^{1}[26]$. Also, it makes use of commercial Lego cylinders, limiting the rapid prototypeability. Secoli et al. developed a powerful three-piston motor (Figure $3.2 \mathrm{~d}$ ), but with a volume of $3000 \mathrm{~cm}^{3}$ it is relatively large [113]. The design of Guo et al. (Figure 3.2e) is innovative and easy to manufacture, but the current prototype has the smallest torque and power of all discussed designs [60].

Besides rotational stepper motors which could drive a spindle or rack-andpinion mechanism to actuate linear motion, true linear stepper motors also have been developed. The authors of this paper, Groenhuis et al. developed two designs of different sizes (Figure 3.2f-g) produced by laser-cutting combined with 3-D printing, delivering up to $24 \mathrm{~N}$ of force. Due to the choice of valves, these have only been tested at speeds up to 20 steps/s, delivering $0.48 \mathrm{~W}$ for the design used in the Stormram 1 robot [46], and $0.15 \mathrm{~W}$ for the more compact design in the Stormram 2 robot [49]. The Stormram 1 and 2 are robotic systems for breast biopsy. Excluding the needle, both of them are MR safe. To our knowledge, no other linear pneumatic stepper motors have been developed, but the generic mechanism has been described in a patent application [16].

None of these motors meet all requirements stated in Section 3.2 for our MR safe robot application. Certain motors have too large dimensions, while others do not meet the force and velocity requirements in an MRI environment. Furthermore, many motors are hard to replicate and therefore a new design is required.

Besides the motors presented in this paper, two other novel motors that meet the stated requirements are published by the same authors [50]. The T26 is a further miniaturized version of the T-63 presented in this paper, while the $\mathrm{C}-30$ is a curved stepper motor similar to the T-26 but in which the rack is curved to make a rotational motion.

\footnotetext{
${ }^{1}$ After communicating with the author, it was found out that the originally claimed $25 \mathrm{~W}$ of power was a miscalculation.
} 


\begin{tabular}{|c|c|c|c|c|c|c|c|c|c|c|c|c|}
\hline $\begin{array}{l}\dot{0} \\
\vdots \\
0 \\
0\end{array}$ & 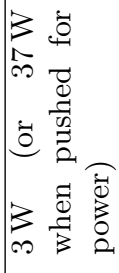 & 3 & 艿 & $\begin{array}{l}3 \\
i n \\
i \\
+i\end{array}$ & 茎 & $\mid$\begin{tabular}{l}
3 \\
$\infty$ \\
\multirow{0}{*}{} \\
0
\end{tabular} & $\mid \begin{array}{l}3 \\
10 \\
-1 \\
0\end{array}$ & 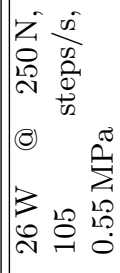 & 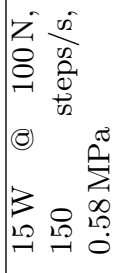 & 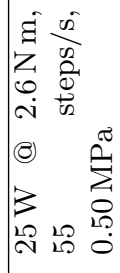 & 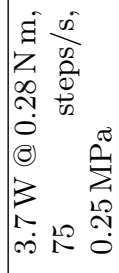 & 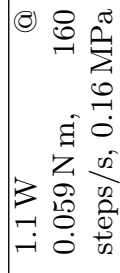 \\
\hline 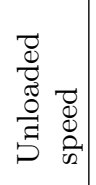 & 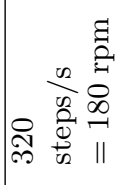 & 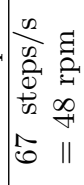 & 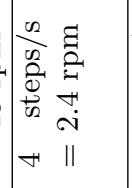 & 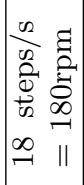 & 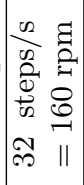 & 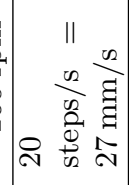 & 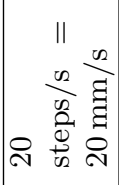 & 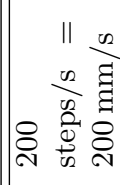 & 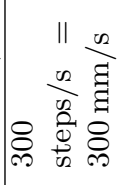 & 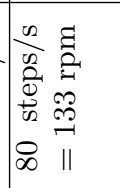 & 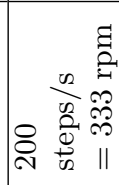 & 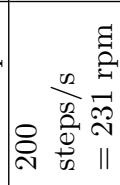 \\
\hline 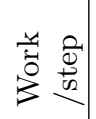 & 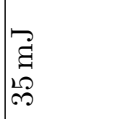 & 急 & 胥 & 急 & $\mid \begin{array}{l}\vec{Z} \\
\dot{a} \\
20 \\
\text { on }\end{array}$ & 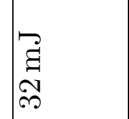 & 胥 & 竎 & 豙 & 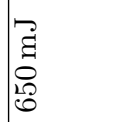 & $\mid \begin{array}{c}\mathfrak{Z} \\
\infty \\
\infty \\
1\end{array}$ & 吾 \\
\hline 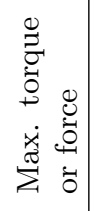 & 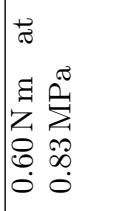 & 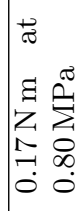 & 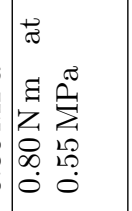 & 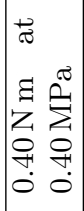 & 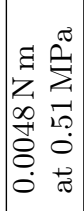 & 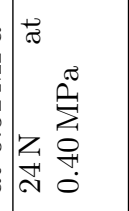 & 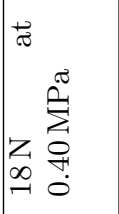 & 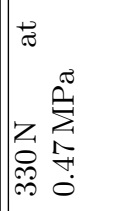 & 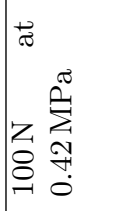 & 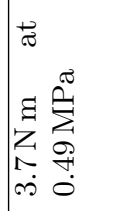 & 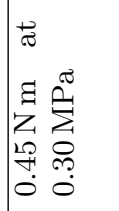 & 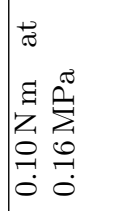 \\
\hline 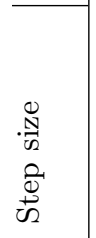 & 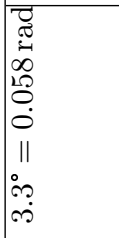 & 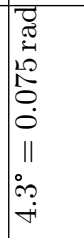 & 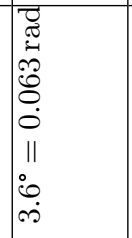 & 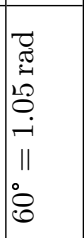 & 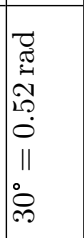 & 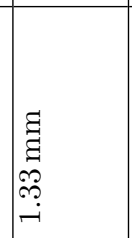 & $\begin{array}{l}\Xi \\
\Xi \\
\Xi \\
0 \\
0 \\
-1\end{array}$ & 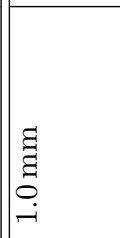 & 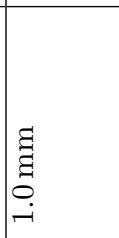 & 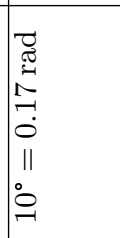 & 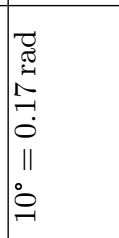 & 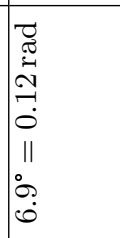 \\
\hline 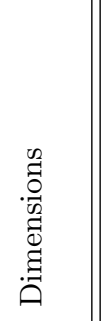 & 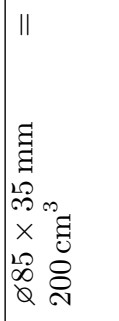 & 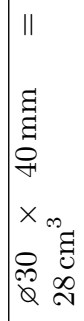 & 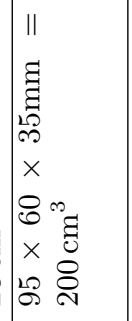 & 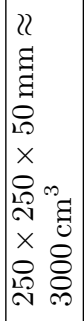 & 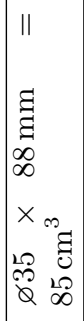 & 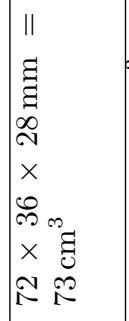 & 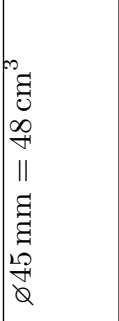 & 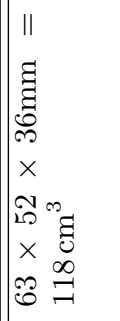 & 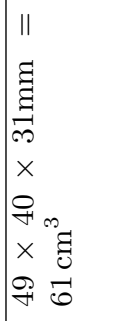 & 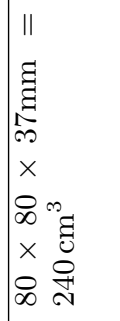 & 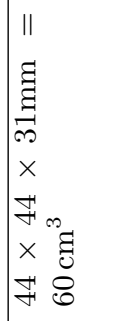 & 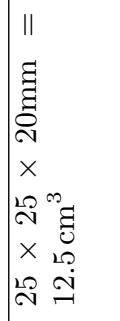 \\
\hline 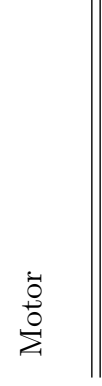 & 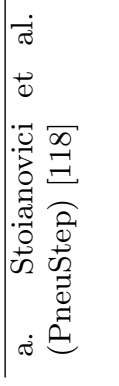 & 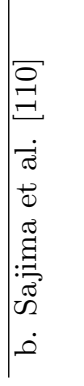 & 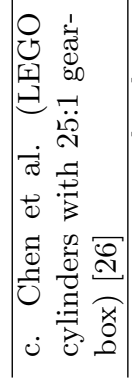 & 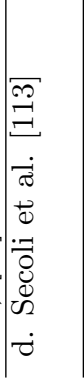 & $\begin{array}{l}\text { तี } \\
+0 \\
0 \\
0 \\
\Xi \\
0 \\
0 \\
0\end{array}$ & 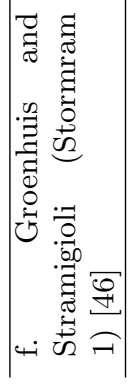 & 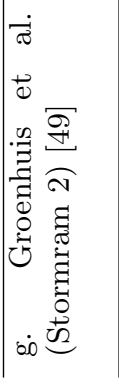 & 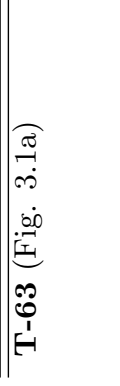 & 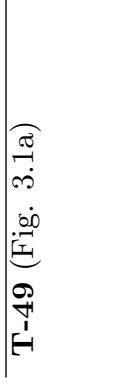 & 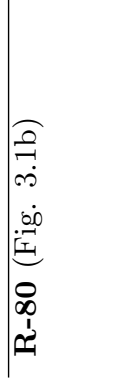 & 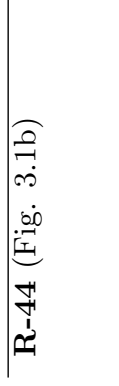 & 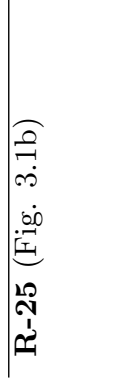 \\
\hline
\end{tabular}




\subsection{Design and production}

A systematic method for constructing novel linear and rotational stepper motors has been developed. Each motor consists of two pistons, which push against a rack (in linear motors) or geared axle (in rotational motors) by a wedge mechanism inside the pistons. Each piston moves back and forth in a double-acting cylinder, therefore a single motor is driven by four pneumatic tubes in total. This section describes the principle of operation, a study on teeth geometry, calculations on valve airflow, and two concrete stepper motor designs.

\subsubsection{Principle of operation}

Figure 3.3a shows five consecutive states of a linear stepper motor (labeled 0 to 4 ), and Figure 3.3b the corresponding piston waveforms and position of the rack. The position of a motor is controlled by driving quadrature-encoded waveforms to the two cylinders.

Only one piston can be fully engaged to the rack (or gear). This is always the piston which was pressurized formerly. The reason is that the piston which was pressurized most recently, temporarily released its grip during its move, allowing the other piston to fully engage to the rack. This is also the reason why the actual position lags by one step from time $t=8$ when piston A switches state. The actual motor position not only depends on the current valve states, but also on the direction of the previous step. This phenomenon is called hysteresis and results in an offset between nominal and actual position, depending on the movement direction. However, this is by design since a consequence is that the motor has zero backlash, even under the presence of finite clearances in the cylinders. Furthermore, it is not envisaged to obtain a smooth motion, or enable any form of microstepping in the operation of the motors.

\subsubsection{Teeth geometry}

The geometry of the teeth determines important properties such as step size, wedge ratio, cylinder length and maximum load. Sharp teeth have a higher wedge ratio resulting higher force output, and allows for smaller step sizes for a given teeth depth, but 3-D printability and mechanical stresses put constraints on this and other design parameters.

For teeth printed with the Ultimaker 2, the step size cannot be smaller than $0.6 \mathrm{~mm}$ due to the $0.4 \mathrm{~mm}$ nozzle of the printer which causes rounding of the teeth.

Additional manufacturing effects such as seams, clearances in mechanical parts and bending under loads up to $0.1 \mathrm{~mm}$, cause additional deviations in the order of $0.2 \mathrm{~mm}$. To obtain a safe margin for such errors, a step size of $1.0 \mathrm{~mm}$ 

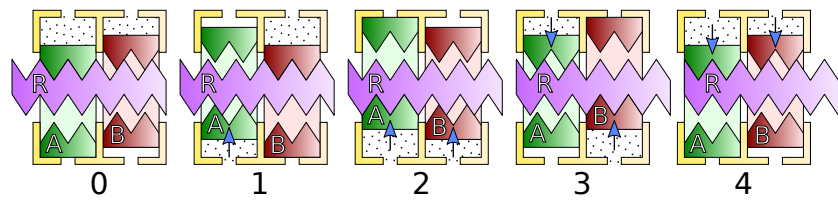

(a) Five consecutive motor states in a linear stepper motor. The pistons (A and B) alternatingly move up and down, pushing the rack

(R) four steps to the right.

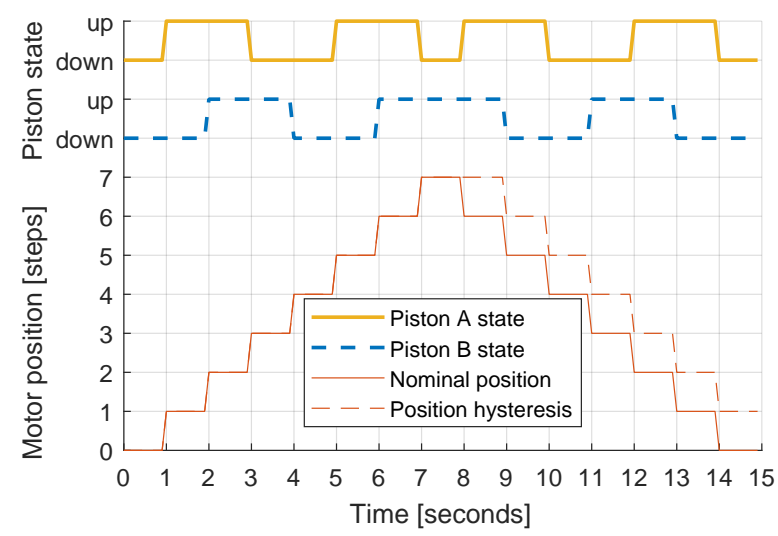

(b) Piston waveforms and corresponding motor position graph, without and with taking hysteresis into account.

Figure 3.3: Operating principle of a linear stepper motor running at $1 \mathrm{~Hz}$, relating piston waveforms to the rack position.

is chosen. As the step size is one quarter of the pitch size, the pitch size is then equal to $4.0 \mathrm{~mm}$.

The teeth depth now determines the tip angle and the wedge ratio. To avoid bending or cracking under high cyclic load, a stress analysis (by simulation and/or measurement) needs to be conducted to determine the maximum load. For a step size of $1.0 \mathrm{~mm}$, the maximal tip deflection under specified loads must not exceed $0.1 \mathrm{~mm}$ and the material stress must not exceed the maximum working stress, taking a sufficient margin of safety into account.

\subsubsection{Valve airflow}

Pressurized air is required to bring a piston into motion. The pressure in each chamber is controlled by a system of remote electromagnetic valves, supplied with system (gauge) pressure $P$. As the motor is placed inside the MRI scanner and electromagnetic valves (which are not MR safe) must be placed outside 


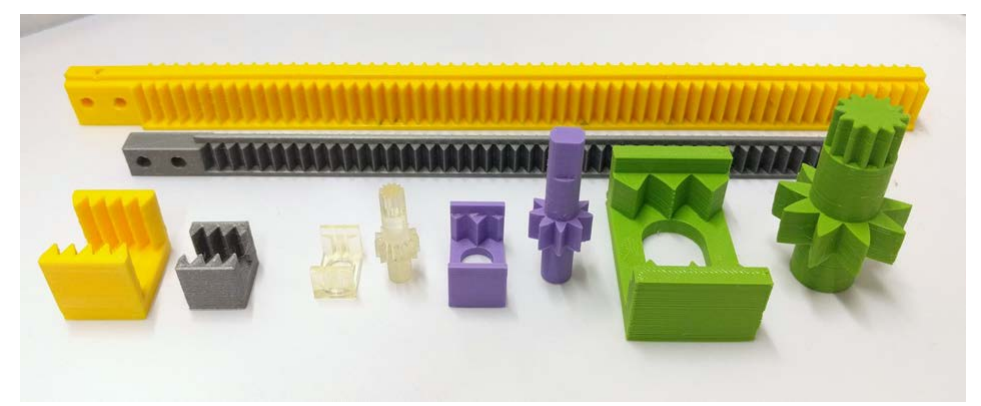

Figure 3.4: Racks, toothed pistons and geared axles for the T-63 (yellow), T-49 (gray), R-80 (green), R-44 (purple) and R-25 (semi-transparent).

the Faraday cage of the MRI scanner, tubes of certain length are needed to connect the motor to the valves. With tube length $L_{t}$, inner diameter $d_{t}$ and cylinder bore volume $V_{c}$, the total air volume $V_{a}$ associated with pressurizing one cylinder bore is:

$$
V_{a}=\frac{\pi}{4} L_{t} d_{t}^{2}+V_{c}
$$

With $L_{t}=5.0 \mathrm{~m}, d_{t}=2.5 \mathrm{~mm}, V_{c}=1.0 \mathrm{~mL}$, we obtain $V_{a}=25.5 \mathrm{~mL}$. With a step size of $1 \mathrm{~mm}$ and a required velocity of $5 \mathrm{~mm} / \mathrm{s}$, a stepping frequency of $5 \mathrm{~Hz}$ is required. The average actual airflow through the valve is then $5 \cdot 25.5=$ $128 \mathrm{~mL} / \mathrm{s}$, or $7.65 \mathrm{~L} / \mathrm{min}$. At a (gauge) pressure of $0.4 \mathrm{MPa}$, the standard airflow is $\frac{0.4}{0.1013} \cdot 7.65=30.2 \mathrm{~L} / \mathrm{min}$. This figure is a lower bound on the nominal valve airflow requirement. As the instantaneous airflow through a valve depends on the pressure drop, and there are also delay and friction effects in the tube, the actual airflow requirement is higher than $30.2 \mathrm{~L} / \mathrm{min}$. Due to the limitation of the complexity of pressurization wave propagation dynamics, measurements were performed instead to assess the pressurization time of a selected valve.

Valves of type Festo MHP2-MS1H-5/2-M5 were selected, which have a specified nominal standard airflow of $90 \mathrm{~L} / \mathrm{min}$ or $1.5 \mathrm{~L} / \mathrm{s}$. The theoretical minimum time required to fill a $25.5 \mathrm{~mL}$ volume to a pressure of $0.4 \mathrm{MPa}$ is then $\frac{0.4}{0.1013} \cdot \frac{25.5}{1500}=67 \mathrm{~ms}$.

\subsubsection{Motor designs}

Five motors have been developed: two linear and three rotational ones. All five motors consist of six different custom components: the housing with top and bottom cover, two identical toothed pistons, four identical silicone seals and one rack or geared axle.

The main design challenges are in designing space-efficient cylinders that have relatively large bores (to provide high forces) and are well sealed (to mini- 
mize leakages), and in transferring the piston force efficiently to the rack or gear. Classic cylindrical-shaped pneumatic cylinders with protruding rods are difficult to rapid prototype, so a different method is used that essentially involves placing the rack or gear right through the pistons themselves, as employed earlier in laser-cut pneumatics [46]. This eliminates the need of a protruding rod, simplifying and compacting the design. Another design choice is the use of rectangular-shaped cylinders, to maximize the force transferred to the rack for the same total motor volume and also for rapid prototypeability.

Figure 3.4 shows racks, gears and pistons of the different motors; it can be observed that the pistons are box-shaped with a cut-out space in its center, and series of teeth facing this cut-out space. Except for the R-25, the housing and covers are held together with M4 nylon screws into the tapped bottom cover, and sealed by blue silicone (Loctite 5926).

The box-shaped pistons are sealed by rectangular silicone rubber seals with slanted edges that are hand-cut from $2 \mathrm{~mm}$ silicone rubber using a 3-D printed cutting guide. For the R-25 motor, seals were laser-cut from $1 \mathrm{~mm}$ silicone rubber for more precise manufacturing due to the smaller size. The seals are preloaded in length and height by approximately $2 \%$. From experimentations, this factor was found to be the optimal tradeoff between minimizing sliding friction and air leakage.

Walls surrounding the rectangular cylinder bores must be thick enough to resist deformation under pressure. The reason is that the bore's crosssectional shape is rectangular, while only circular cylinders have an optimal cross-sectional area to perimeter ratio. For $20 \mathrm{~mm}$ high cylinders, a wall thickness of $8 \mathrm{~mm}$ was chosen. FEM simulations have shown that deformation under pressure of $0.4 \mathrm{MPa}$ stays below $0.1 \mathrm{~mm}$, to keep air leakage to a minimum. Airflow measurements are performed to quantify this amount.

All rigid parts of the T-63 motor were produced using the Ultimaker 2 . This 3-D printer produces parts using fused filament fabrication. The material used is Makerpoint PLA (Makerpoint Holding, Wageningen, The Netherlands). From its specifications, the Young's modulus $3.3 \mathrm{GPa}$ and ultimate tensile strength is $110 \mathrm{MPa}$. Using a safety factor of 4 , the design stress is $26 \mathrm{MPa}$.

Parts printed by fused filament fabrication method are not isotropic, which implies that actual tensile strength depends on the orientation of the load. Also, surface roughness varies significantly: of the bottom-facing faces, the lowermost one is smooth because it is printed directly on the glass print bed, while all other bottom-facing faces are rough. The top-facing faces are moderately rough, but the topmost one can be polished smooth by grinding. The side faces are all grooved due to the layered printing technique. For moving parts to slide with relatively low friction, surfaces have to match in smoothness: moderately rough surfaces must be coupled with smooth ones, and grooved surfaces must slide 


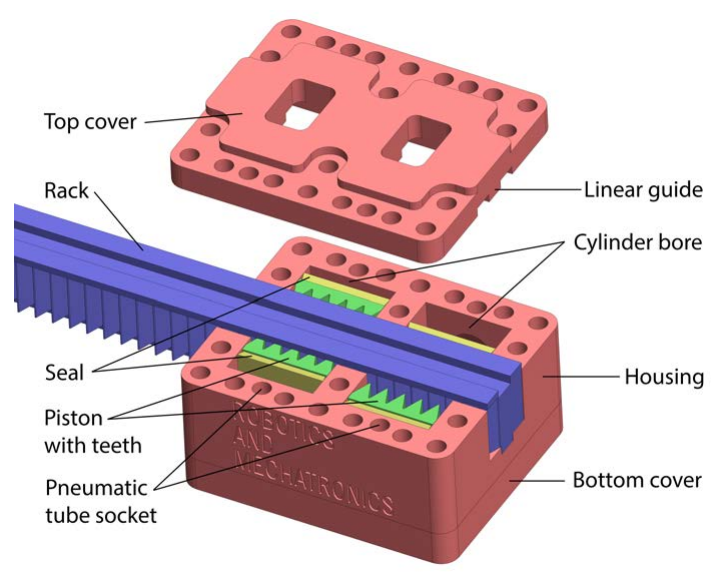

Figure 3.5: T-63 view, with lifted top cover.

in the direction of the grooves. Vaseline (petroleum jelly) is used to lubricate the parts, minimizing friction and wear.

Because solid 3-D printed parts are generally not fully airtight due to the presence of small pores, a dilute solution of transparent ABS in acetone is applied to its walls after which these pores are sealed.

\subsubsection{T-63 linear motor}

The T-63 motor (Figure 3.1a, top) is a linear motor with dimensions $63 \times 52 \times$ $36 \mathrm{~mm}$. Figure 3.5 shows a CAD drawing of the motor.

The rack is constrained by linear guide rails. $4 \mathrm{~mm}$ tubes are mechanically clamped by purposely misaligning the pneumatic tube socket holes in the top cover and housing. For reasons described in Section 3.3.2, a teeth pitch of $4 \mathrm{~mm}$ was chosen. The teeth depth, which is a tradeoff between high wedge ratio and short cylinder stroke, was chosen to be $5 \mathrm{~mm}$, resulting in a teeth tip angle of $43.6^{\circ}$.

The bore's cross-sectional area is $20 \mathrm{~mm} \times 20 \mathrm{~mm}=400 \mathrm{~mm}^{2}$. When supplied with a pressure $P$, a force $F_{p}=P \cdot A=4 \cdot 10^{-4} \cdot P$ is exerted on the piston. The teeth act on the rack by means of a wedge mechanism. A piston displacement of $2.5 \mathrm{~mm}$ results in $1 \mathrm{~mm}$ rack displacement, so the wedge ratio is $\alpha=\frac{2.5 \mathrm{~mm}}{1 \mathrm{~mm}}=2.5$ and the force transferred from piston to the rack is increased by this factor. Ignoring friction, the output force $F_{r}$ satisfies $F_{r}=\alpha F_{p}=10^{-3} \cdot P=1000 \mathrm{~N} / \mathrm{MPa}$. At a pressure of $P=0.3 \mathrm{MPa}$, the output force will then be $F_{r}=300 \mathrm{~N}$. The actual output force is less due to friction losses between moving parts (seals, pistons, rack), which can be determined experimentally. 


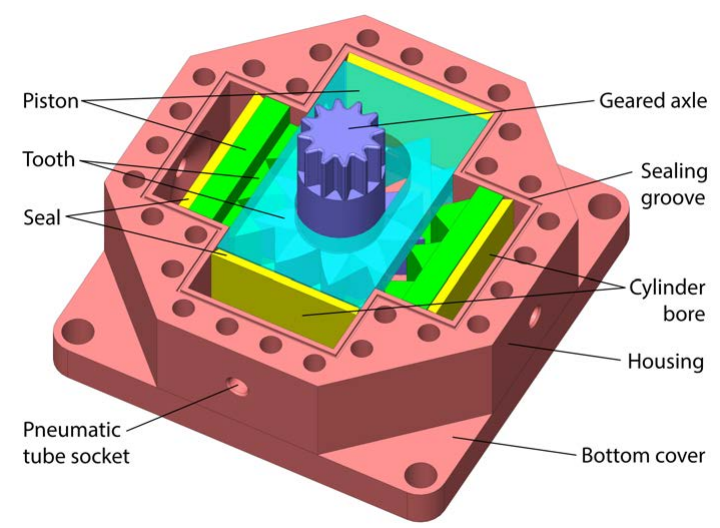

Figure 3.6: R-80 view, without top cover, and one piston made transparent.

The T-63 serves as a proof-of-concept, but does not meet the dimensionality requirement of a medical robot application. Therefore, a miniaturized version of the T-63 has been developed, resulting in the T-49 design. This motor has a smaller cross-sectional area and teeth depth, and has a length of $49 \mathrm{~mm}$.

\subsubsection{R-80 rotational motor}

The R-80 is a rotational stepper motor. See Figure 3.6 for the CAD design and Figure $3.1 \mathrm{~b}$ (left) for the realization. The top and bottom covers were lasercut from transparent acrylic plates to make the internal parts visible from the outside, all other rigid parts were 3 -D printed in PLA with the Ultimaker 2 machine. The two pistons act on a gear to rotate it around, requiring the piston movement to be perpendicular to the gear tip's motion profile. Therefore, the two identical pistons have to be placed in a cross configuration, embracing the axle, and the pistons have a slotted hole for the axle.

Dimensions are $80 \mathrm{~mm} \times 80 \mathrm{~mm} \times 37 \mathrm{~mm}$ and the bore size is $30 \times 20 \mathrm{~mm}=$ $600 \mathrm{~mm}^{2}$, resulting in $60 \mathrm{~N}$ of force per $0.1 \mathrm{MPa}$ pressure. The gear has nine teeth, with circular pitch $40^{\circ}$ and teeth depth $8 \mathrm{~mm}$. The step size is $10^{\circ}$, equal to one quarter of the circular pitch. Upon a piston movement of $x=4 \mathrm{~mm}$, the gear rotates by $\beta=10^{\circ}$. The torque $T$ as function of pressure $P$ can be found using the work balance $T \beta=F x=P A x$, so: $T=P \frac{6 \cdot 10^{-4} \cdot 4 \cdot 10^{-3}}{10 \cdot \pi / 180} \approx$ $1.38 \cdot 10^{-5} P=13.8 \mathrm{Nm} / \mathrm{MPa}$. For a pressure of $0.3 \mathrm{MPa}$, the theoretical output torque (ignoring friction losses) is $4.1 \mathrm{Nm}$.

In an optimal situation, the piston teeth surface keeps maximal contact with the gear when sliding on it. However, unlike in the linear stepper motor designs, there is no ideal solution for the shape of the planar contact curve. Curved segments that keep full contact when sliding, must be circular or linear. 


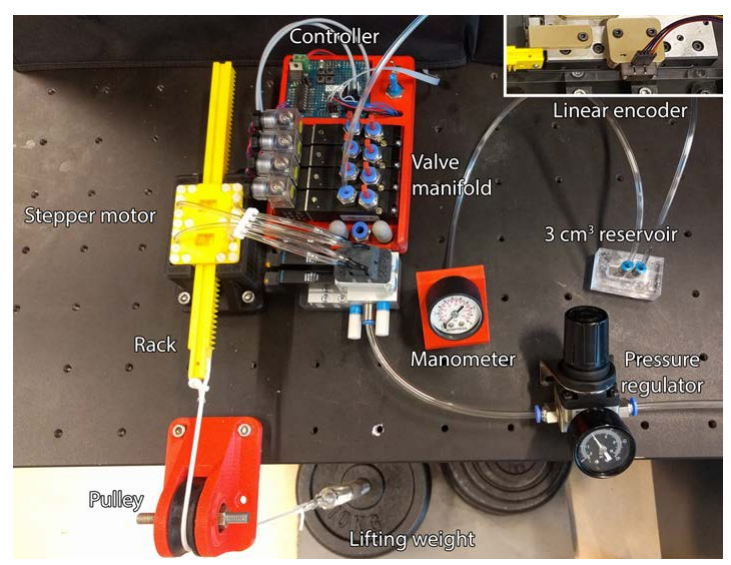

Figure 3.7: Setup for force measurements.

But if the angle between the tangent and radial line is to be kept constant, the curve must be a logarithmic spiral which does not have a constant radius of curvature. Combined with the fact that a single tooth has to transfer the full load, the consequence is that the piston and rack have a relatively small effective contact surface area, compared with linear stepper motors.

As with the T-63, a miniaturized version of the R-80 has been developed to meet the dimensional requirements for applications in MR safe robotics. The R-44 motor (Figure 3.1b, middle) measures $44 \mathrm{~mm}$ and was printed with the Ultimaker 2 in PLA material, while the R-25 motor (Figure 3.1b, right) measures $25 \mathrm{~mm}$ and was printed with the Stratasys (Stratasys Ltd., Eden Prairie, MN, USA) Objet Eden260 in FullCure720 material.

\subsection{Measurements}

\subsubsection{Methods and setups}

Twelve experiments have been conducted, to characterize various aspects of the five motors. Measurement setups were constructed for different motor characterization, teeth strength, tube propagation delay and MR safety tests. This section describes the methods and setups used for the different experiments.

Figure 3.7 shows the general measurement setup for evaluating different motors. Constant force loads on linear stepper motors are generated by lifting combinations of weights of known mass using a pulley. Winches with radius $20 \mathrm{~mm}$ or $50 \mathrm{~mm}$ allow to convert these forces to torques to load rotational stepper motors. The system pressure is adjusted using a manual pressure regulator and measured with an analog or digital manometer. The stepping frequency is 
controlled by an analog turn knob connected to the Arduino controller of the valve manifold, its frequency setting was communicated over a serial interface to a laptop and displayed on its screen. Acceleration is kept below $0.4 \mathrm{~m} / \mathrm{s}^{2}$, to reduce the variation in rope tension. The Arduino keeps an internal step counter and allows to define two preset positions for feedforward position control. The directly actuated valves are of type Festo MHP2-MS1H-5/2-M5, with average response time of $1.8 \mathrm{~ms}$ and nominal flow rate of $90 \mathrm{~L} / \mathrm{min}$. For transient positional measurements, an incremental optical linear encoder of type EM2-0-1000-I (US Digital, Vancouver, WA, USA) with a resolution of $0.00635 \mathrm{~mm}$, and an absolute angular encoder of type Novotechnik P4501A502 (Novotechnik, Southborough, MA, USA), sampled with the 10-bit ADC of an Arduino for an effective resolution of $0.34^{\circ}$, were used. For measuring precise linear displacements, the mechanism of a digital caliper (length $150 \mathrm{~mm}$ and accuracy $0.01 \mathrm{~mm}$ ) was rigidly attached to the rack and table by means of custom 3 -D printed parts.

\section{Teeth strength}

To study the optimal teeth shape for the T-63 motor, stress and deformations were simulated by applying a $100 \mathrm{~N}$ load on three different tooth shapes, with depth $5 \mathrm{~mm}, 8 \mathrm{~mm}$ and $12 \mathrm{~mm}$, and all having pitch $4 \mathrm{~mm}$ and width $14 \mathrm{~mm}$. To validate the simulation results, a deflection measurement experiment with teeth depth $12 \mathrm{~mm}$ was also carried out, in which the tooth was loaded with a $10 \mathrm{~kg}$ mass. Furthermore, the maximum load on R-80's gear was investigated by simulations.

\section{Tube propagation delay}

To study the delay and rise/fall times, the pressure step response for $4 \mathrm{~mm}$ tubes with inner diameter $2.5 \mathrm{~mm}$ and lengths $0.20 \mathrm{~m}, 1.0 \mathrm{~m}$ and $5.0 \mathrm{~m}$ were measured by applying square waveforms on its input and recording the transient output pressure. This was performed both without and with a $3.0 \mathrm{~cm}^{3}$ reservoir at its end, resulting in six different waveforms from which the delay and rise/fall times were derived and analyzed.

\section{Force/torque versus pressure}

(all motors): The weight was varied and the pressure adjusted to the lowest level where the motor could just lift the given weight without missing steps, at low frequency $(1 \mathrm{~Hz})$ and short tubes $(0.20 \mathrm{~m})$. Also, the effect of varying tube length on force/torque was studied by measuring the minimum required pressure for different tube lengths. 


\title{
Maximum unloaded speed
}

(all motors): Stepping frequency was increased until the motor misses steps. This could be observed by moving between two preset step count positions, and checking that the actual preset positions do not drift away. Short $(0.20 \mathrm{~m})$ tubing was used and pressure was adjusted such that the stepping frequency could be pushed to the highest level.

\section{Force versus pressure at different stepping frequencies}

(T-49 only): The weight and stepping frequency were varied and the pressure was adjusted to the lowest level where the motor could just lift the given weight without missing steps. Short tubes $(0.20 \mathrm{~m})$ were used.

\section{Force versus speed for different tube lengths}

(T-49 only): The pressure was fixed at $0.55 \mathrm{MPa}$ and the maximum stepping frequency was determined for all combinations of weight $(0 \mathrm{~N}, 35 \mathrm{~N}, 70 \mathrm{~N}, 100 \mathrm{~N})$ and tube length $(0.20 \mathrm{~m}, 1.0 \mathrm{~m}, 5.0 \mathrm{~m})$.

\section{Maximum power}

(all motors): Possible combinations of stepping frequencies, weight load and pressure were tested to find the maximum power operating point for the motor.

\begin{abstract}
Airflow
(T-49 only): The standard airflow was measured by filling a $14 \mathrm{~L}$ tank to a pressure of $0.6 \mathrm{MPa}$, and then operating a motor until the tank pressure drops to $0.5 \mathrm{MPa}$, while the system pressure was maintained at $0.4 \mathrm{MPa}$. By recording the duration of motor operation, the flow rate is derived. This is first performed at zero frequency (idle) to derive the amount of leakage, and then for three different tube lengths $(0.20 \mathrm{~m}, 1.0 \mathrm{~m}$ and $5.0 \mathrm{~m})$ in combination with three different stepping frequencies, to derive the air volume consumption per step.
\end{abstract}

\section{Overshoot}

(T-63 and R-80): In order to investigate possible overshooting of the motors, the transient displacement of the R-80 and T-63 were recorded with encoders and subsequently analyzed. 


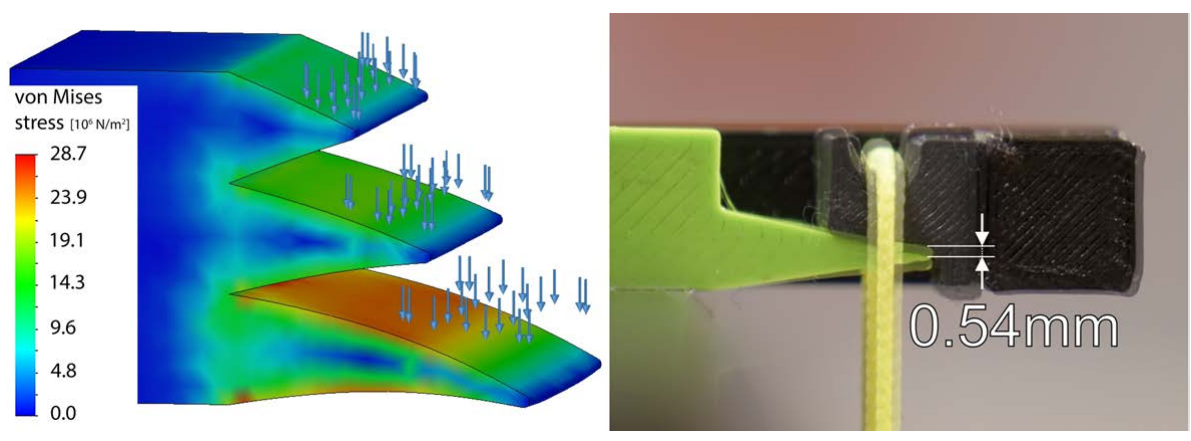

Figure 3.8: Left: Stress simulation on three teeth, with depths $5 \mathrm{~mm}, 8 \mathrm{~mm}$ and $12 \mathrm{~mm}$. Deformation scale is 5.22. Right: Tip displacement measurement. Tooth depth is $12 \mathrm{~mm}$. Photos with and without $100 \mathrm{~N}$ load are superimposed.

\section{Positional accuracy}

(T-63 only): Precise actual displacements were measured over a range of setpoint positions, and its differences analyzed.

\section{Durability}

(T-63 only): The motor was operated for one hour, lifting and lowering a $120 \mathrm{~N}$ load over a distance of $120 \mathrm{~mm}$ in a cycle of ten seconds, delivering $6 \mathrm{~W}$ of power in a duty cycle of $50 \%$ at $0.4 \mathrm{MPa}$ pressure. The piston mass was measured before and after the test, to quantify the amount of wear in the pistons.

\section{MR classification}

(T-63 only): The appropiate MR compatibility classification is primarily determined by analyzing the complete list of materials that the motor is made of. Additionally, MRI scans of the motor inside an Esaote G-scan Brio 0.25T MRI scanner next to a geometric phantom (water solution containing $\mathrm{NiCl}_{2}$ and $\mathrm{NaCl}$ ) were taken and analyzed.

\subsubsection{Results}

This section describes the results of the twelve experiments described in the previous section.

\section{Teeth strength}

Figure 3.8 (left) shows the result of the stress simulation, as carried out with Solidworks (Dassault Systems Solidworks Corp., MA, USA). The $5 \mathrm{~mm}$ tooth 


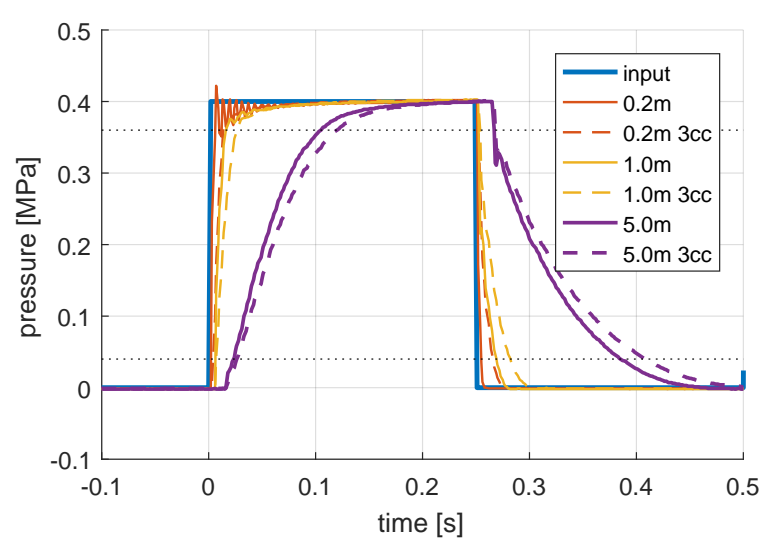

Figure 3.9: Pressure step response for three different tube lengths, with and without $3 \mathrm{~cm}^{3}$ reservoir.

has a displacement at the tip of less than $0.03 \mathrm{~mm}$ and a maximum stress of 9.6 MPa. The $8 \mathrm{~mm}$ tooth has a displacement of $0.10 \mathrm{~mm}$ and maximum stress $14 \mathrm{MPa}$. Finally, the $12 \mathrm{~mm}$ tooth has an excessive displacement of $0.36 \mathrm{~mm}$ and a maximum stress of $29 \mathrm{MPa}$.

To validate the simulation, a measurement was conducted by applying a $100 \mathrm{~N}$ load on a $12 \mathrm{~mm}$ tooth. Figure 3.8 (right) shows that the measured deflection at the tip was $0.54 \mathrm{~mm}$. This is $50 \%$ higher than the simulated value of $0.36 \mathrm{~mm}$, which could be attributed to the inhomogenity of the material resulting from the layered $3-\mathrm{D}$ printing process. Using this figure, the effective Young's modulus of the material is $2.2 \mathrm{GPa}$.

Simulations on the R-80 gear have shown that a $330 \mathrm{~N}$ load on one tooth causes a stress of $45 \mathrm{MPa}$ which exceeds the working stress. The maximum allowable force was found to be $190 \mathrm{~N}$, which corresponds to a torque of $2.3 \mathrm{~N} \mathrm{~m}$ on its axle.

\section{Tube propagation delay}

The step response of the piston pressure was measured for six different cases. Figure 3.9 shows the pressure graphs as function of time. For each length, the tube was either closed at the end, or connected to a $3.0 \mathrm{~cm}^{3}$ reservoir at the end (simulating the cylinder stroke volume of the T-63 motor). It can be observed that longer tubes have slower response. In case of the shortest tube without reservoir, the pressure response shows underdamped oscillatory behaviour.

Table 3.2 lists the average delay and rise/fall time for each of the six cases. The delay is caused by the switching time of the MHP2-MS1H-5/2-M5 valve, 


\begin{tabular}{c|c|c|c} 
Case & Volume & Delay & Rise/fall time \\
\hline $0.2 \mathrm{~m}$ & $1.0 \mathrm{~mL}$ & $3 \mathrm{~ms}$ & $4 \mathrm{~ms}$ \\
$0.2 \mathrm{~m}+3 \mathrm{~cm}^{3}$ & $4.0 \mathrm{~mL}$ & $3 \mathrm{~ms}$ & $10 \mathrm{~ms}$ \\
$1.0 \mathrm{~m}$ & $4.9 \mathrm{~mL}$ & $7 \mathrm{~ms}$ & $13 \mathrm{~ms}$ \\
$1.0 \mathrm{~m}+3 \mathrm{~cm}^{3}$ & $7.9 \mathrm{~mL}$ & $7 \mathrm{~ms}$ & $25 \mathrm{~ms}$ \\
$5.0 \mathrm{~m}$ & $24.5 \mathrm{~mL}$ & $18 \mathrm{~ms}$ & $100 \mathrm{~ms}$ \\
$5.0 \mathrm{~m}+3 \mathrm{~cm}^{3}$ & $27.5 \mathrm{~mL}$ & $18 \mathrm{~ms}$ & $116 \mathrm{~ms}$
\end{tabular}

Table 3.2: Average delay and rise/fall time measurements for three different tube lengths.

which is $1.8 \mathrm{~ms}$, plus the propagation delay caused by the speed of sound, which is $340.3 \mathrm{~m} / \mathrm{s}$. In case of the $5.0 \mathrm{~m}$ tube, the measured delay of $18 \mathrm{~ms}$ is consistent with the theoretical value of $0.0018+\frac{5.0}{340.3}=16 \mathrm{~ms}$. The measured rise/fall times are approximately proportional to the tube volume; the measured time of $100 \mathrm{~ms}$ for the $5.0 \mathrm{~m}$ tube is consistent with the earlier calculated value of $67 \mathrm{~ms}$, because actual airflow drops when pressure approaches its setpoint.

\section{Force/torque versus pressure}

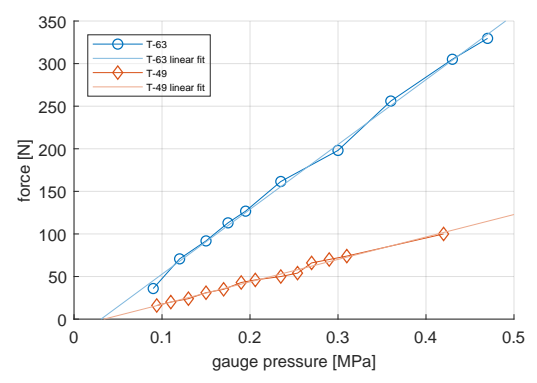

(a) T-63 and T-49 force versus pressure.

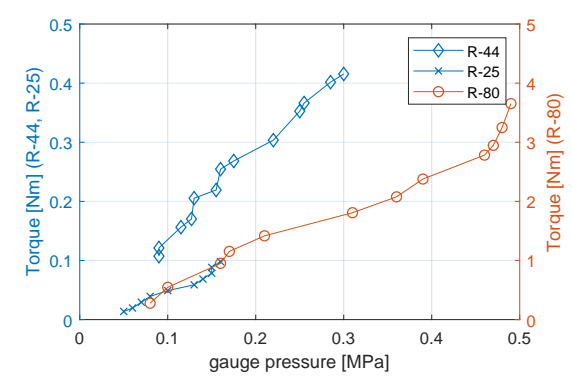

(b) R-80, R-44 and R-25 torque vs. pressure.

Figure 3.10: Force/torque versus pressure measurement results for linear and rotational stepper motors.

Figure 3.10a shows the force characteristics of both linear stepper motor designs, and Table 3.1 the highest measured force or torque for each motor, for $0.20 \mathrm{~m}$ short tubes. The T-63 is capable of delivering $330 \mathrm{~N}$ of force, while the T-49 was tested up to $100 \mathrm{~N}$.

The linear fitting equation of the T-63 force graph is $F=761 \cdot 10^{-6} \mathrm{P}-$ $23.3 \mathrm{~N}$, with $F$ in Newton and $P$ in Pascal. Its coefficients are the slope $761 \mathrm{~N} / \mathrm{MPa}$ and offset $-23.3 \mathrm{~N}$. The coefficient of determination is $R^{2}=$ 0.9973, which indicates its linearity. Compared with the theoretical model of $1000 \mathrm{~N} / \mathrm{MPa}$, we see that the T-63 has a mechanical efficiency of $\frac{761 \mathrm{~N} / \mathrm{MPa}}{1000 \mathrm{~N} / \mathrm{MPa}}=$ 
$76 \%$. This is the ratio of work performed by the rack, to work performed by the pressurized air on the piston in the cylinder. The remaining work is lost due to friction in sliding parts, and converted to heat.

The T-49 force graph has a linear fitting equation of $F=264 \cdot 10^{-6} P-8.9 \mathrm{~N}$. Its coefficients are the slope $264 \mathrm{~N} / \mathrm{MPa}$ and offset $-8.9 \mathrm{~N}$. The coefficient of determination is $R^{2}=0.9920$, which also indicates its linearity. The mechanical efficiency is $\frac{264 \mathrm{~N} / \mathrm{MPa}}{400 \mathrm{~N} / \mathrm{MPa}}=66 \%$, which is lower than the efficiency of the T-63. Comparing the force/pressure graphs of the T-63 and T-49, we see that the T-63 motor is a factor $\frac{761 \mathrm{~N} / \mathrm{MPa}}{264 \mathrm{~N} / \mathrm{MPa}} \approx 2.9$ stronger than the T-49 motor.

When $5.0 \mathrm{~m}$ long tubes are used, the T- 63 was found to consistently require an additional $0.02 \mathrm{MPa}$ of pressure to lift a given weight, which can be attributed to the pressure drop in long tubes at steady airflow resulting from small air leakages in the motor.

Figure 3.10b gives the torque characteristics of the three rotational stepper motor designs. The R- 80 is capable of exerting $3.7 \mathrm{~N} \mathrm{~m}$ of torque, at a higher load (approximately $4 \mathrm{Nm}$ ) one tooth broke off. The simulated stress at that point was approximately $45 \mathrm{MPa}$, so this is the effective yield strength under dynamic loading. Using a design stress of $26 \mathrm{MPa}$, the maximum torque reduces to $2.3 \mathrm{Nm}$.

The R-44 exerted over $0.48 \mathrm{~N}$ m of torque before breaking down. The R-25 was tested up to $0.10 \mathrm{Nm}$, which is also close to its point of breakdown.

Again, all characteristic curves in Figure 3.10b are approximately linear.

The mechanical efficiency of the R-80 is $\frac{6.91 \mathrm{Nm} / \mathrm{MPa}}{13.8 \mathrm{Nm} / \mathrm{MPa}}=50 \%$, the mechanical efficiency of R-44 is $\frac{1.44 \mathrm{Nm} / \mathrm{MPa}}{2.29 \mathrm{Nm} / \mathrm{MPa}}=63 \%$ and R-25's mechanical efficiency is $\frac{0.68 \mathrm{Nm} / \mathrm{MPa}}{1.03 \mathrm{Nm} / \mathrm{MPa}}=66 \%$. Although smaller pistons have a larger perimeter-tosurface ratio, the mechanical efficiency seems not to be directly related to the size of the motor. Variations in the choice of materials, the manufacturing process and specific teeth geometries seem to have a more dominant effect on the friction and the relative mechanical efficiencies.

\section{Maximum unloaded speed}

The maximum unloaded stepping frequencies are listed in Table 3.1. The T-49 is the fastest with 320 steps/s, and R-80 the slowest with 80 steps/s.

\section{Force versus pressure at different stepping frequencies}

Figure 3.11a shows the force/pressure relationship of the T-49 motor for different stepping speeds. At low speeds, the graph follows the one in Figure 3.10a. For stepping frequencies up to $150 \mathrm{~Hz}$, the maximum force of $100 \mathrm{~N}$ can still be exerted if the pressure is increased. But at higher stepping frequencies $(200 \mathrm{~Hz}$ and higher), the maximum force is significantly reduced. 

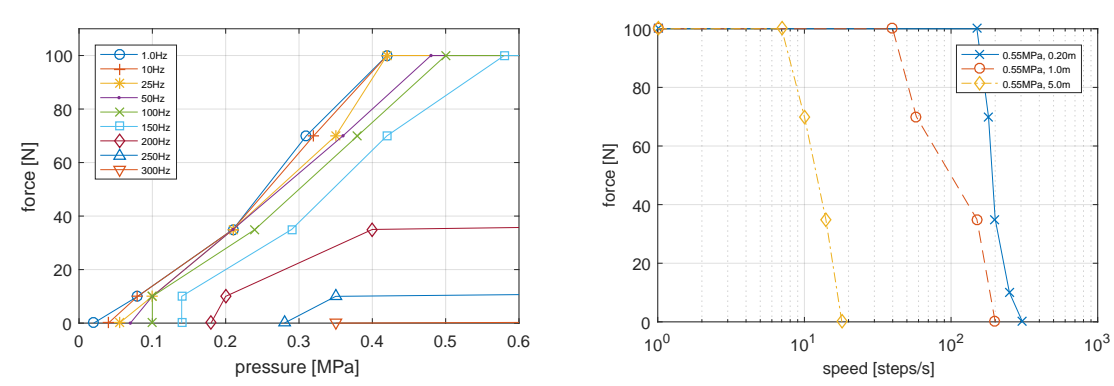

(a) T-49 force vs. pressure for different stepping (b) T-49 force vs. speed for different tube speeds. lengths.

Figure 3.11: T-49's relationships between force, pressure, speed and tube length.

\begin{tabular}{c|c|c|c} 
Tube length & Measured air volume & Tube+bore volume & Efficiency \\
\hline $0.20 \mathrm{~m}$ & $(13 \pm 2) \mathrm{mL}$ & $2.1 \mathrm{~mL}$ & $12 \%$ \\
$1.0 \mathrm{~m}$ & $(30 \pm 8) \mathrm{mL}$ & $6.0 \mathrm{~mL}$ & $5.2 \%$ \\
$5.0 \mathrm{~m}$ & $(140 \pm 10) \mathrm{mL}$ & $26 \mathrm{~mL}$ & $1.1 \%$
\end{tabular}

Table 3.3: Air consumption per step in the T-49 motor and tube+bore volume, and pneumatic efficiency, for different tube lengths.

\section{Force versus speed for different tube lengths}

Figure 3.11b shows T-49's force-speed relationship for different tube lengths (outer diameter $4 \mathrm{~mm}$, inner diameter $2.5 \mathrm{~mm}$ ), at a pressure of $0.55 \mathrm{MPa}$. As expected, using $5.0 \mathrm{~m}$ long tubes restricts operation to lower stepping frequencies (order of $10 \mathrm{~Hz}$ ) than when $0.20 \mathrm{~m}$ or $1.0 \mathrm{~m}$ tubes are used (order of $100 \mathrm{~Hz}$ ).

\section{Maximum power}

The maximum power found for each motor is given in Table 3.1. Short tubes $(0.20 \mathrm{~m})$ were used. The pressure was generally set to $0.5 \mathrm{MPa}$, except for the R-44 and R-25 where the pressure was lower in order not to damage the gears. The T-63 and R- 80 turned out to be the most powerful motors, both delivering around $25 \mathrm{~W}$. The R-44 delievered a stable $3.7 \mathrm{~W}$ of power. When the frequency was increased from $75 \mathrm{~Hz}$ to $125 \mathrm{~Hz}$, delivering $6.2 \mathrm{~W}$ for a short time, the gear broke down. The R-25 managed to deliver $1.1 \mathrm{~W}$ of power, and also broke down when the frequency was increased further.

\section{Airflow}

The T-49's airflow at standstill and a pressure of $0.4 \mathrm{MPa}$ was measured to be $(250 \pm 10) \mathrm{mL} / \mathrm{s}$ on average (normalized to atmospheric pressure). This is the 


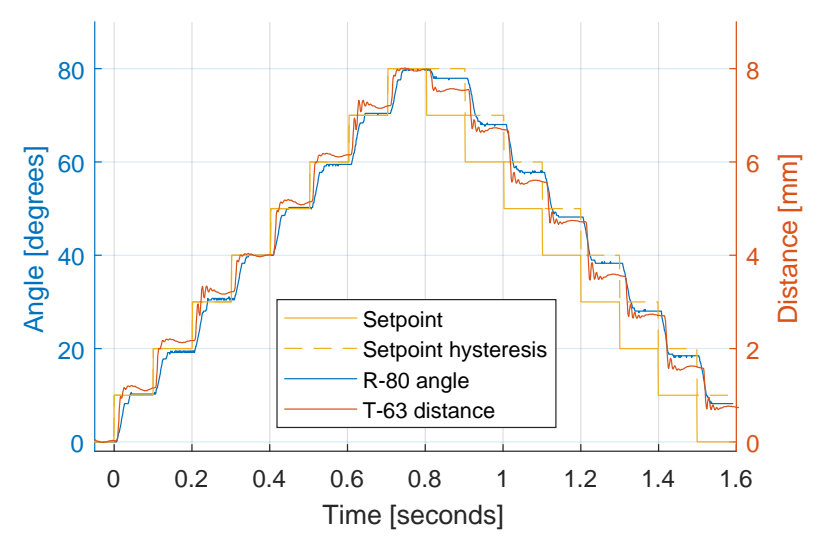

Figure 3.12: Transient angle measurements of the T-63 and R-80 motors.

amount of air leaking through the housing and covers, and along the seals of the T-49. The leakage of other motors varies from $34 \mathrm{~mL} / \mathrm{s}$ for the R-25, to $424 \mathrm{~mL} / \mathrm{s}$ for the T-63.

The measured air volume consumption (normalized to atmospheric pressure) per step by the T-49 motor is listed in the second column of Table 3.3. This amount of air is needed to increase the pressure in the tube, cylinder bore and valve by $0.4 \mathrm{MPa}$, and is indeed just over four times the calculated geometric volume. As the effective piston stroke distance is $2.0 \mathrm{~mm}$ and piston area is $196 \mathrm{~mm}^{2}$, the effective stroke volume is $0.392 \mathrm{~mL}$, requiring $1.57 \mathrm{~mL}$ of air. The pneumatic efficiency is the ratio between this volume and the measured step volume, and listed in the last column of Table 3.3. As expected, the efficiency is highest for short tubes. For a given tube length and valve airflow, these figures determine the maximum stepping frequency. For $5.0 \mathrm{~m}$ tubes and a nominal airflow of $90 \mathrm{~L} / \mathrm{min}=1.5 \mathrm{~L} / \mathrm{s}$, the maximum stepping frequency is $8.9 \mathrm{~Hz}$ (ignoring delay and friction effects in the tubes). This is consistent with measurements on the pressure step response and maximum stepping frequency.

\section{Overshoot}

Figure 3.12 shows the transient measurements of the T-63 and R-80 motors. In forward motion, the overshoot of the R- 80 is observed to be $0.3^{\circ}$ on average, which is in the same order as the sensor resolution. The T-63 shows oscillatory behaviour in different frequencies resulting from dynamics in the mechanical system, in particular the masses of the lifting weight and the linear rail for the optical encoder. The overshoot is approximately $0.1 \mathrm{~mm}$ on average. Both overshoots are one order of magnitude smaller than the step size. 


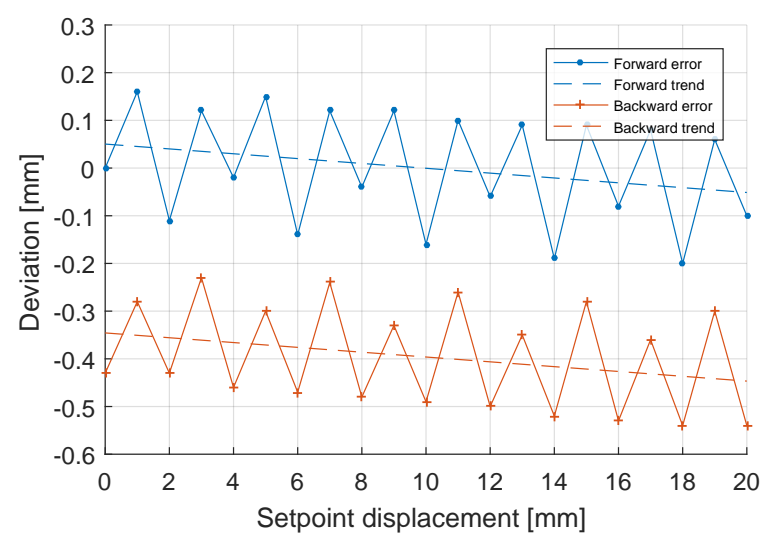

Figure 3.13: Displacement error of the T-63 motor, in forward and backward direction.

In reverse direction, the transients have the same characteristics and magnitude as in forward direction. Additionally, a hysteresis can be observed as expected. For the R-80, the offset between setpoint (without hysteresis) and measured position is $8^{\circ}-9^{\circ}$, while for the T- 63 the offset is $0.6-0.7 \mathrm{~mm}$. On average, the magnitude of hysteresis is $60 \%-80 \%$ of the step size. The graph is consistent with Figure 3.3b.

\section{Positional accuracy}

Figure 3.13 shows the displacement error of the T-63 motor as function of the setpoint position (with hysteresis) between $0 \mathrm{~mm}$ and $20 \mathrm{~mm}$. The following observations can be made:

- The error depends on the movement direction. When moving backward, the controller assumes hysteresis of one step, but there is a bias of $-0.40 \mathrm{~mm}$ in the measured position which can be attributed to clearances and material stresses in the direction of movement.

- The trendline shows that the deviation decreases with $0.0051 \mathrm{~mm}$ per $\mathrm{mm}$ displacement $(0.51 \%)$. A possible cause is the shrink factor for PLA associated with the 3-D printing process, when the part is cooled off inside the printer. This could be compensated for by increasing the dimensions by a certain factor, so that the dimensions after shrinkage are more accurate.

- The deviation is periodic with an interval of four steps. At odd-numbered setpoint displacements, the deviation is consequently above the trendline, while at even-numbered ones the deviation is below the trendline. Apparently, there is a slight asymmetry causing an offset of $0.21 \mathrm{~mm}$ between 
Ch 3: Rapid Prototyping High-Performance MR Safe Pneumatic Stepper 66

Motors

\begin{tabular}{l|l} 
Part & Material \\
\hline Housing, pistons, rack & PLA \\
Screws & Nylon \\
Seals & Silicone rubber \\
Tubing & Polyurethane \\
Glue, sealant, lubricant & Loctite $406 / 770 / 5926$, petroleum jelly
\end{tabular}

Table 3.4: List of materials of T-63

the deviations of both pistons, which can be caused by 3 -D printing effects.

- Taking the trendline and periodic deviations into account, the standard deviation error from that calibrated displacement is below the measurement error of $0.01 \mathrm{~mm}$.

Next, a repeatability test was performed. The rack was moved ten times between setpoint $0 \mathrm{~mm}$ and $100 \mathrm{~mm}$. The measured positions at the lower endpoint was consistently $0.00 \mathrm{~mm}$, while at the upper endpoint it measured either $99.15 \mathrm{~mm}$ or $99.16 \mathrm{~mm}$. Its difference is within the measurement error of $0.01 \mathrm{~mm}$.

The measurements show that there is a significant, but highly repeatable deviation between setpoint and actual displacement. The RMS (root mean square) error from the trend line is $0.11 \mathrm{~mm}$. After calibration of the eight different motor phases (four forward and four backward), the repeatability is in the order of $0.01 \mathrm{~mm}$.

\section{Durability}

The T-63 motor was operated during one hour, lifting and lowering a $120 \mathrm{~N}$ load over a distance of $120 \mathrm{~mm}$ once every ten seconds, performing 86400 steps in total. Before the test, the masses of the pistons were measured to be $5.91 \mathrm{~g}$ and $5.81 \mathrm{~g}$, respectively. After operating the motors for one hour, the measured masses were $5.91 \mathrm{~g}$ and $5.80 \mathrm{~g}$, respectively. The weight measurement error is $0.01 \mathrm{~g}$, so there was no significant change in the masses of both pistons. Visual inspection also revealed no sign of wear. The motor did not skip a single step in the test. This particular motor can therefore operate for at least one hour under the tested load, with an indication that much longer runs are possible.

\section{MR classification}

Table 3.4 lists all the materials contained in the T-63. None of the listed materials are conducting, metallic and/or ferromagnetic, and therefore the T63 can be classified to be MR safe. 


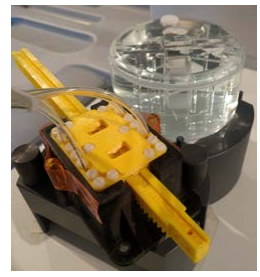

(a) Setup

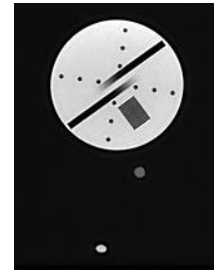

(b) No motor

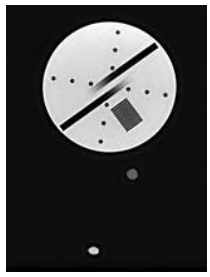

(c) Running

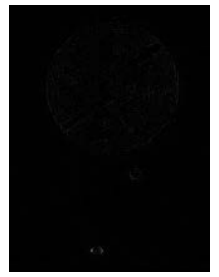

(d) Diff.

Figure 3.14: MRI scans of the T-63 motor next to a geometric phantom, without motor and with running motor. The motor is placed between the two fiducials next to the phantom. Image $(\mathrm{d})$ is the difference between (b) and (c).

\begin{tabular}{c|c|c|c} 
Situation & Mean intensity $\mu$ & Standard deviation $\sigma$ & SNR $[\mathrm{dB}]$ \\
\hline No motor & 1211 & 18.5 & 36.3 \\
Idle motor & 1204 & 19.9 & 35.7 \\
Running motor & 1190 & 18.1 & 36.4
\end{tabular}

Table 3.5: Signal-to-noise ratio of bright patch in phantom, for three situations.

The MR safe classification implies that the device has no potential for interaction with the magnetic field, and this was also verified with an actual MRI experiment. Figure 3.14 shows the setup and MRI scans of a geometric phantom and a motor holder with two fiducials attached to it. Three conditions were tested: without motor, with idle motor placed in the holder and with T-63 continuously running at $7 \mathrm{~Hz}$.

A $20 \times 20$ pixel bright patch in the image of the phantom was selected, in the proximity of the motor. The average signal intensity and standard deviation of this patch were evaluated and listed in Table 3.5, along with the derived signal-to-noise ratio (SNR) in decibels, for all three conditions. There is no significant change in SNR when a running motor is present, compared to the other situations.

Figure 3.14d shows the image difference between the first and third situation. There is no change visible in the image of the phantom, on the scale of one pixel $=0.94 \mathrm{~mm}$. This implies that the presence and operation of the motor do not cause any measurable distortions in the image.

\subsubsection{Discussion}

Table 3.1 lists the developed motor's specifications alongside with state-of-theart metal-free bidirectional pneumatic stepper motors. The T-63 motor is able to deliver $330 \mathrm{~N}$ of force, which is over ten times stronger than the two other linear stepper motors found in literature [46, 49]. The T-63 did not miss any steps and the teeth showed no signs of wear after an hour long test, which means 
that the motor can be feed-forward controlled and is durable. Simulations also showed that its $5 \mathrm{~mm}$ deep teeth can individually handle $100 \mathrm{~N}$ loads without excessive stress or deformations.

The R- 80 rotational motor managed to deliver $3.7 \mathrm{Nm}$ when pushed for torque, or $2.3 \mathrm{~N} \mathrm{~m}$ when taking maximum material stresses into account, which is still three times stronger than the most powerful rotational motors found in literature [113, 118].

Transient positional measurements on the T- 63 and R- 80 motors have shown that hysteresis is present as expected, and that the overshoot is one order of magnitude smaller than the step size. This overshoot can be considered insignificant compared to the discretization error, which is half the step size. For the T-63 motor which has a $1.0 \mathrm{~mm}$ nominal step size, deviations are present in the order of $0.1 \mathrm{~mm}-0.5 \mathrm{~mm}$, due to various manufacturing inaccuracies resulting from 3-D printing. The repeatability was found to be $0.01 \mathrm{~mm}$, so a well-calibrated motor has good positional accuracy.

Comparing motors in terms of delivered power is not straightforward. There are no standardized test protocols, so wattage measurements depend on the actual setup used such as tubing dimensions and valve specifications. The PneuStep delivers $3 \mathrm{~W}$ during normal operation, but also claimed to have delivered $37 \mathrm{~W}$ when pushed for power. The R- 80 and T-63 both managed to deliver $25 \mathrm{~W}$, but the long-term durability of the T-63 was evaluated at $6 \mathrm{~W}$ only. Additional measurements are needed to investigate the durability of the motor at different wattages to find out the long-term maximum operating limits. On the other hand, if we could choose stronger non-metallic materials such as PEEK or ceramics, which PneuStep also does, then the absolute operating limits would be significantly extended and the measured $25 \mathrm{~W}$ of power could then be considered to be within normal operating range.

The T-63 and R-80 are too bulky for applications in MR safe robotics. Therefore, miniaturized versions of these motors have been developed as well. As the amount of work performed per step is proportional to the stroke displacement volume, downsizing one motor design results in a performance scaled down roughly proportional to its linear dimensions.

The T-49 satisfies all requirements, delivering $100 \mathrm{~N}$ of force. One application is the Stormram 3, an MR safe robotic system for breast biopsy [51]. The R-44 and R-25 rotational motors are also compact, but not robust yet. The gears need to be re-dimensioned or constructed from stronger materials to avoid breaking down at excessive loads. The main reason is that one single tooth has to bear the full load, while in the linear motors the total load is evenly distributed over multiple teeth.

Certain applications require the valves to be placed a minimum distance away from the motors. In case of MR safe robotics, this distance is in the order of $5 \mathrm{~m}$ (except when placing the valves in a shielded enclosure within the 
Faraday cage of the MRI scanner). The maximum stepping frequency of the T49 motor over a distance of $5 \mathrm{~m}$ was measured to be $7 \mathrm{~Hz}$ when maximum force is needed, which is consistent with pressure step response measurements. If this frequency is too low, then valves with higher airflow and tubes with larger inner diameter are required to increase the stepping frequency to the desired level. For distances of $1 \mathrm{~m}$, the maximum stepping frequency was measured to be $40 \mathrm{~Hz}$, and for very short tubes the T-49 motor can be operated at up to $150 \mathrm{~Hz}$ while maintaining the maximum force of $100 \mathrm{~N}$.

After the development of Stoianovici's PneuStep in 2007, multiple attempts have been made to develop metal-free pneumatic stepper motors that are compact, powerful and easy to manufacture, with limited success. Many of the previously developed motors are relatively weak or too bulky, due to the small bore sizes, inefficient mechanics, excessive leakage or other reasons.

In contrast, the designs presented in this paper show radically improved specifications and are fully rapid prototypeable. The main improvement is that the cylinders make more effective use of the available space: each cylinder bore's width is approximately one-third of the total housing length, and the bore height is approximately half the motor height, resulting in relatively large piston head surfaces resulting in high forces. The rectangular-shaped bore makes the different components rapid prototypeable, and the multifunctional pistons require no additional components to transfer the pneumatic forces to the rack or gear.

\subsection{Conclusion}

Five stepper motors have been developed: two linear and three rotational ones, in sizes ranging from $25 \mathrm{~mm}$ to $80 \mathrm{~mm}$. The T-63 and R-80 motors have shown to be significantly stronger than any other non-metallic pneumatic motor found in literature, while the T-49, R-44 and R-25 are small enough to be applied to MR safe robotics and therefore satisfy all requirements. All motors are easy to manufacture by rapid prototyping. The presented design method involving box-shaped pistons that embrace a rack or gear inside a space-efficient housing, has shown to be the key concept that is likely to advance the field of metal-free pneumatic stepper motors towards a higher level. 



\section{Dual-Speed MR Safe Pneumatic Stepper Motors}

V. Groenhuis, F. Siepel, and S. Stramigioli. Dual-Speed MR Safe Pneumatic Stepper Motors. In Proceedings of Robotics: Science and Systems, Pittsburgh, Pennsylvania, 2018. doi: 10.15607/rss.2018.xiv.030 


\subsection{Abstract}

In breast cancer detection it is essential to perform precise interventions to determine the diagnosis. Robotic systems actuated by MR safe pneumatic stepper motors could improve accuracy to target the tumor. The achievable accuracy or speed is limited due to long pneumatic tubes connecting the motors to the electromagnetic valves in the control room. This paper presents the design of two dual-speed stepper motors in order to solve this limitation.

The linear motor measures 50x32x14 mm (excluding racks) and has step sizes $1.7 \mathrm{~mm}$ and $0.3 \mathrm{~mm}$. The maximum speed under load is $20 \mathrm{~mm} / \mathrm{s}$, measured force is $24 \mathrm{~N}$ and positioning accuracy is $0.1 \mathrm{~mm}$. The rotational motor measures $\varnothing 30 \times 32 \mathrm{~mm}$ (excluding axles) and has step sizes $10^{\circ}$ and $12.9^{\circ}$. Under load its maximum angular speed is $229^{\circ} / \mathrm{s}$ or $38.2 \mathrm{RPM}$, maximum torque is $74 \mathrm{Nmm}$ and positioning accuracy is $1^{\circ}$. By operating the valves in a coordinated way high-speed and precise position control can be achieved. With these specifications the motors have high potential to actuate MR safe surgical robots.

\subsection{Introduction}

In breast cancer screening, medical imaging is used by a radiologist to explore abnormalities. When a suspicious lesion is found a biopsy may be required for accurate histological evaluation. In this procedure a biopsy needle is inserted to the location of the suspicious lesion under imaging guidance after which the tissue is sampled and examined. While most lesions are well visible on mammography (x-ray) and ultrasound, a small part is only detectable on MRI. The current MRI-guided breast biopsy procedure is inaccurate and inefficient resulting in long procedure times, additional tissue damage and possibly a false negative biopsy [29, 129].

A robotic system placed inside the MRI scanner allows precise needle operations under near-realtime MRI guidance and could improve the breast biopsy procedure. The construction and actuation of such a robot needs special consideration to avoid interference with the strong magnetic field of the MRI scanner. In the current terminology as defined by the ASTM F2503-13 standard three possible classifications are given to medical equipment: "MR unsafe", "MR conditional" and "MR safe". The strongest classification, "MR safe", is exclusively assigned to devices which are entirely constructed of non-metallic, non-magnetic and non-conductive materials, as determined from its material decomposition rather than MR compatibility tests.

Energy for actuation has to be transferred mechanically to the robot by means of pneumatics, hydraulics or cables. All three methods have its own advantages and disadvantages. A hydraulic actuator utilizes the incompress- 


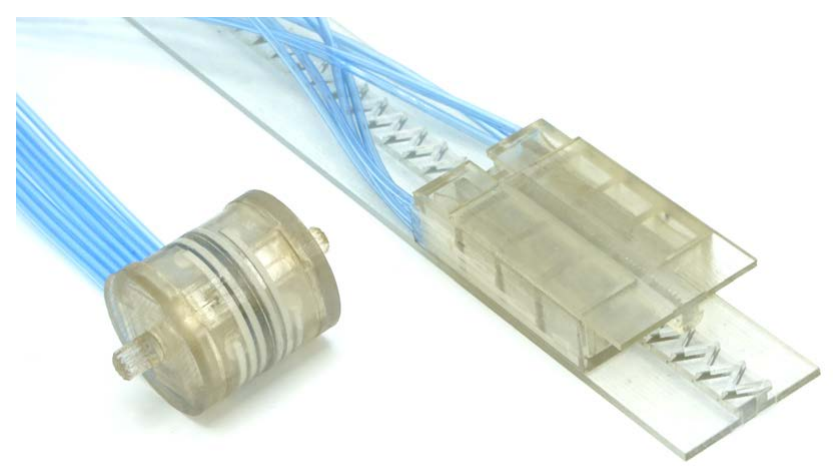

Figure 4.1: Photographs of rotational (left) and linear (right) dual-speed pneumatic stepper motors.

ibility of fluid to transmit forces and displacements over long distances. Perfect sealing of all parts is required as leaking liquid is troublesome [133], making it difficult to produce parts by rapid prototyping techniques. Actuation by Bowden cables and/or pulley systems have been proven effective in many areas of research and engineering: forces and displacements are transferred by pushing, pulling and/or twisting a cable inside the tube. The main challenge involved is to overcome the static friction, especially when the cable has numerous bends between the robot and controller [70]. Pneumatic systems utilize pressurized air as the medium which has the advantages that it is abundant in hospitals, small leaks are acceptable and long transmission distances are possible without significant drop in supplied pressure. An important drawback is that air is compressible: while a single pneumatic cylinder could be used as an actuator [41], precise position control of the piston is difficult and the only well-defined positions are the two end-stop positions. The air compressibility issue can be mitigated by utilizing the stepper motor mechanism: two or more pneumatic cylinders drive a rack or gear in discrete steps, resulting in a pneumatic stepper motor $[46,55,110]$ on which this research is based.

The relevant characteristics of an MR safe motor are size, force, accuracy and speed. The motor must fit within a device alongside the patient inside the MRI scanner, so the size must be in the order of a few centimetres. The maximum force must be at least $20 \mathrm{~N}$ to allow inserting a biopsy needle in dense tissue. The speed should be sufficient to move the end-effector to a specified location within approximately ten seconds in order not to delay the biopsy procedure. Finally, sub-millimeter accuracy and precision are required to allow targeting of millimeter-sized lesions. 


\subsubsection{State-of-art}

Several MR safe robotic systems driven by pneumatic stepper motors have been demonstrated in literature. Stoianovici et al. [118] demonstrated the MrBot driven by PneuStep motors, a rotational stepper motor with three phases and optical position feedback. The time needed to align the needle guide with the target site is 1:36 $\pm 1.24 \mathrm{~min}$ [120]. Bomers et al. [15] developed the RCM (remote-controlled manipulator), a robotic system for transrectal prostate biopsy and driven by helical pneumatic stepper motors. The mean manipulation time for needle guide movement was measured to be 5:48 min. Groenhuis et al. [55] developed the Stormram 4, which requires up to 1:30 min to manipulate the needle to the commanded position.

All these robots require more than one minute to move to the target position, delaying the biopsy procedure. The low speed is a result of the submillimeter step sizes combined with low stepping frequencies limited by the $5 \mathrm{~m}$ to $10 \mathrm{~m}$ tubes that connect the motors to MR-unsafe valve manifolds outside the Faraday cage of the MRI scanner.

\subsubsection{Analysis of maximum stepping frequency}

The maximum frequency of a pneumatic stepper motor is primarily limited by tube dimensions and valve specifications. In order to move a piston pneumatically one chamber has to be pressurized and the opposite chamber (if any) de-pressurized, both involving the displacement of a certain volume of air through the valve. As the internal volume of a pneumatic tube $\left(24.5 \mathrm{~cm}^{3}\right.$ for $5 \mathrm{~m}$ long, $2.5 \mathrm{~mm}$ inner diameter tubes) is an order of magnitude higher than the cylinder stroke of a stepper motor (order of $1 \mathrm{~cm}^{3}$ ), the tube also has characterstics of a reservoir and the airflow is not constant across the length of the tube, complicating theoretical analysis.

Two different valves are considered. The MHP2 (Festo MHP2-MS1H-5/2M5, Festo AG \& Co. KG, Esslingen am Neckar, Germany) is a direct-acting solenoid valve with a specified switching time of $2 \mathrm{~ms}$. The PV5211 (PneumatiekVoordeel PV5211-24VDC-1/8, TechniComponents B.V., Waalwijk, The Netherlands) is an internally-piloted valve with a specified switching time of under $50 \mathrm{~ms}$. In the PV5211 valve the delay between the switching of the pilot and main valves does not necessarily affect the rise time of the valve, instead it primarily contributes to a phase shift in its frequency response.

The flow rate is restricted by the $2.5 \mathrm{~mm}$ orifice diameters of the valve and tubes. This results in a theoretical maximum flow rate of $343 \pi \times 0.00125^{2} \mathrm{~m}^{3} / \mathrm{s}=$ $1680 \mathrm{~cm}^{3} / \mathrm{s}=101 \mathrm{~L} \mathrm{~min}^{-1}$. The specified nominal flow rates for both valves is approximately $90 \mathrm{~L} \mathrm{~min}^{-1}=1500 \mathrm{~cm}^{3} / \mathrm{s}$ for a system pressure of $0.3 \mathrm{MPa}$ to $0.5 \mathrm{MPa}$ and a pressure drop of $0.1 \mathrm{MPa}$ across the valve, which are consistent with the calculated maximum flow rates. 
Ignoring switching time of the valve the minimum time needed to pressurize a volume of $25 \mathrm{~cm}^{3}$ is $\frac{25}{1500} \mathrm{~s} \approx 17 \mathrm{~ms}$. This lower bound cannot be significantly reduced by using differently-sized valves or tubes, as the minimum travel time for a pocket of air through a $5 \mathrm{~m}$ tube is $\frac{5}{343} \mathrm{~s}=15 \mathrm{~ms}$. This sets a strict upper bound of $\frac{1}{0.015} \mathrm{~Hz}=67 \mathrm{~Hz}$ for the stepping frequency. The actual bandwidth is lower due to friction and dynamics in the system.

Yang et al. [135] approximated the dynamics of a $9 \mathrm{~m}$ long, $3.2 \mathrm{~mm}$ inner diameter tube as a first-order system with bandwidth $5.1 \mathrm{~Hz}$ and time delay $27 \mathrm{~ms}$. Groenhuis and Stramigioli [47] experimentally observed a bandwidth of $10 \mathrm{~Hz}$ when using $5 \mathrm{~m}$ long, $2.5 \mathrm{~mm}$ inner diameter tubes. Higher stepping frequencies are accompanied with a loss of force or torque. Stoianovici et al. [118] implemented position feedback using optical waveguides in order to optimize the stepping frequency for a given load and also account for missing steps.

\subsubsection{Approach}

The aim of this paper is to achieve higher accuracy and speed in pneumatic stepper actuators suitable for MR safe surgical robots. The approach is to combine two singular stepper motors with different step sizes into one housing in a space-efficient way. By simultaneously operating both the large-step side and the small-step side in constructive directions, maximum speed is achieved which is the sum of the individual speeds. Smaller steps can be made by operating the small-step side only or by operating both sides in opposite directions. Even smaller net displacements may be achieved by performing a combination of multiple large and small steps. No position feedback is employed to keep the design as compact and simple as possible. Instead, correctness of controlled position is guaranteed when the motor is not overloaded.

One linear and one rotational dual-speed stepper motor have been developed to demonstrate the different geometries and design parameters and also provide solutions for different types of kinematic joints. The linear motor actuates a prismatic joint while the rotational motor can directly actuate a revolute joint. The step sizes in the linear motor are substantially different while the step sizes of the rotational motor are close to each other.

\subsection{Materials and Methods}

\subsubsection{Linear motor design}

Figure 4.2 shows an exploded view of the dual-speed linear motor. It is based on the design by Groenhuis et al. [51, 55] and consists of a housing (red) with four dual-acting pneumatic cylinders (green) sealed by $10 \times 10 \mathrm{~mm}$ square-shaped seals (yellow) acting on two racks (purple). The large-step side consisting of the 


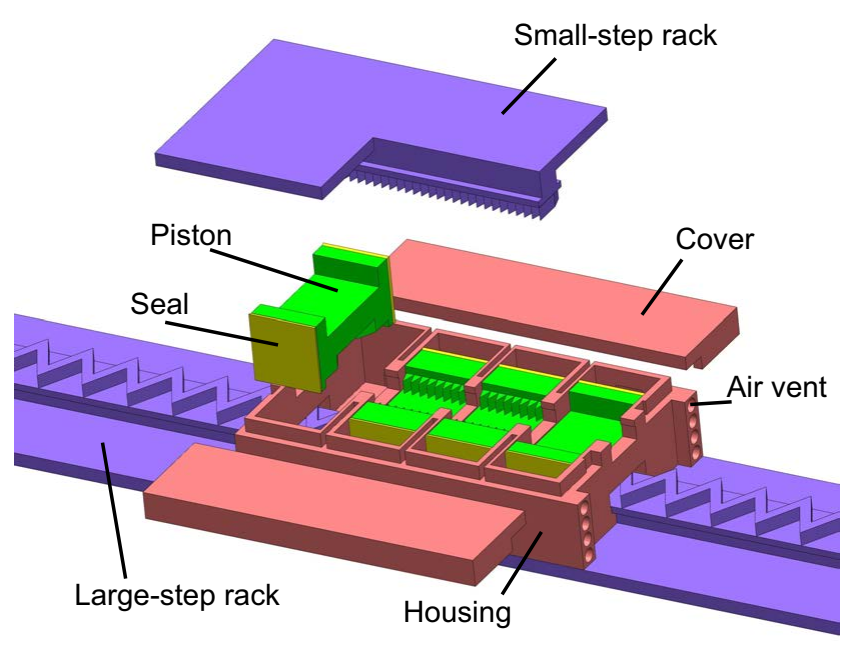

Figure 4.2: Exploded view of linear motor. The pistons (green) slide back and forth inside the box-shaped cavities of the housing (red) sealed by the square seals (yellow). The two outermost pistons have large teeth on the bottom, driving the large-step rack (purple). The two innermost pistons actuate the small-step rack on top.

outermost two cylinders drives the long rack on the bottom which has a teeth pitch of $6.8 \mathrm{~mm}$ and a teeth depth of $6 \mathrm{~mm}$. The small-step side consisting of the innermost two cylinders drives the short rack on the top which has a teeth pitch of $1.2 \mathrm{~mm}$ and a teeth depth of $2 \mathrm{~mm}$. The linear stepper motor measures $50 \times 32 \times 14 \mathrm{~mm}$ excluding racks.

The force exerted by one piston at a pressure of $0.3 \mathrm{MPa}$ is $0.3 \times 10^{6} \times$ $0.01^{2} \mathrm{~N}=30 \mathrm{~N}$. The teeth in the large-step side have a mechanical advantage of $\frac{2 \times 6}{6.8}=1.76$ so the force exerted by the large-step rack (ignoring friction losses) is $1.76 \times 30 \mathrm{~N}=53 \mathrm{~N}$. For the small-step side the advantage ratio is $\frac{2 \times 2}{1.2}=3.33$ resulting in a theoretical force of $3.33 \times 30 \mathrm{~N}=100 \mathrm{~N}$.

The range of motion is determined by the length of the longer rack. The shorter rack is primarily used to enhance positioning accuracy. As 17 small steps result in the same displacement $(5.4 \mathrm{~mm})$ as three large steps, the minimum range of motion for the small-step rack to reach all positions is $5.4 \mathrm{~mm}$. Excess range may still be useful for reaching the desired target position in a lower number of steps.

\subsubsection{Rotational motor design}

Figure 4.3 shows an exploded view of the dual-speed rotational motor. The number and cross-sectional area of the cylinders are identical to that of the 


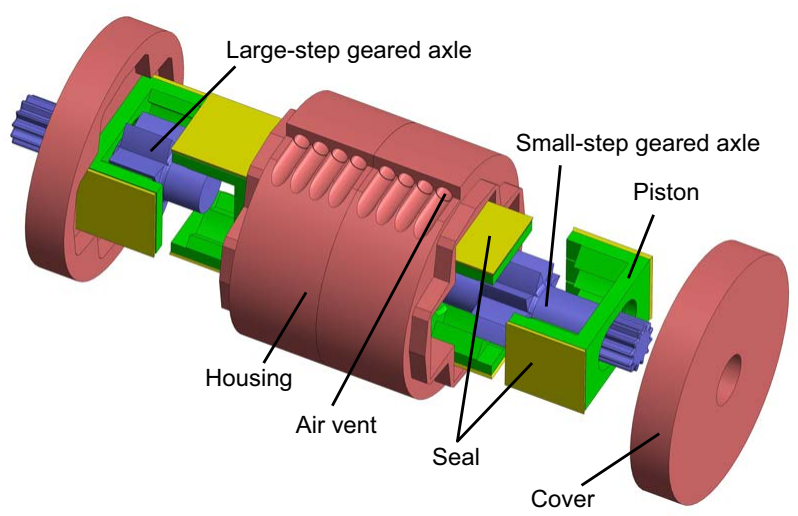

Figure 4.3: Exploded view of rotational motor. The pistons (green) act on the geared axles (purple). The large-step axle gear (left) has seven teeth and takes 28 steps per revolution. The small-step axle gear (right) has nine teeth, taking 36 steps per revolution.

linear motor, but these are placed in a cross configuration and the teeth act on geared axles instead of racks. The axle of the large-step side has seven teeth resulting in a step angle of $\frac{90}{7}=12.86^{\circ}=0.224 \mathrm{rad}$, while the small-step axle has nine teeth and a step angle of $10^{\circ}=0.175 \mathrm{rad}$. The teeth depth on both sides is $2.5 \mathrm{~mm}$. Excluding axles, the motor dimensions are $\varnothing 30 \mathrm{~mm} \times 32 \mathrm{~mm}$.

The piston force at a pressure of $0.3 \mathrm{MPa}$ is also $30 \mathrm{~N}$. For the large-step side this results in a theoretical torque of $\frac{30 \times 0.0025}{2 \times 0.224} \mathrm{~N} \mathrm{~m}=167 \mathrm{~N} \mathrm{~mm}$, while for the small-step side the theoretical torque is $\frac{30 \times 0.0025}{2 \times 0.175} \mathrm{~N} \mathrm{~m}=214 \mathrm{~N} \mathrm{~mm}$.

In a typical application one of the axles is fixed to an inertial reference frame. Actuation of this side will cause the housing to revolve around the axle. The range of motion of this side is constrained by the amount of available space to wrap and un-wrap the pneumatic tubes, which must be at least $90^{\circ}$ to be able to reach all setpoints. The other side can rotate continuously if not constrained by the range of motion of the actuated kinematic joint.

Figure 4.4 shows a schematic cross-sectional view of the large-step rotational unit through a sequence of five states. The two pistons (red and green) both make contact with the geared axle (purple), but only the piston which was pressurized formerly establishes full contact with the gear and determines the position. This eliminates backlash but also causes hysteresis when the direction is reversed. When the four cylinder chambers are pressurized in a particular sequence the gear performs step-wise rotational movements in the desired direction. Figure 4.5 shows a similar operating sequence for a generic linear stepper motor unit. 


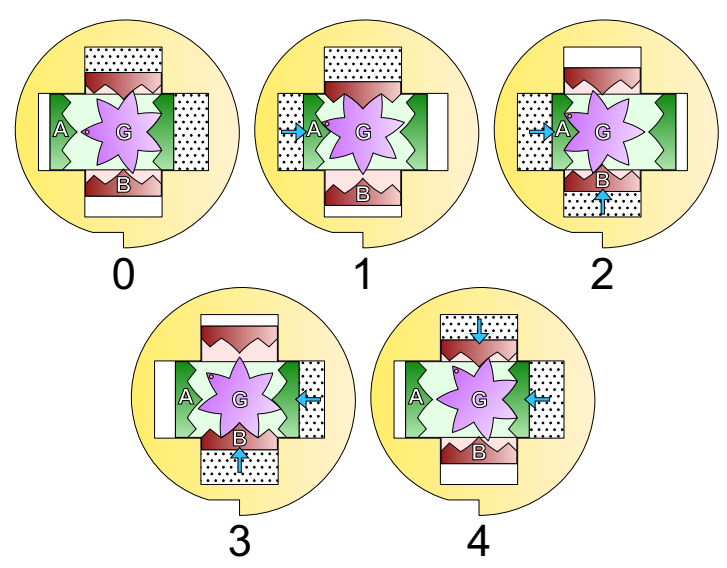

Figure 4.4: Five consecutive states of the large-step side of the rotational motor with the housing (yellow), geared axle (purple) and pistons (red and green). A step is performed by pneumatically moving one piston to the opposite side. After performing four steps the gear has rotated by one-seventh revolution.
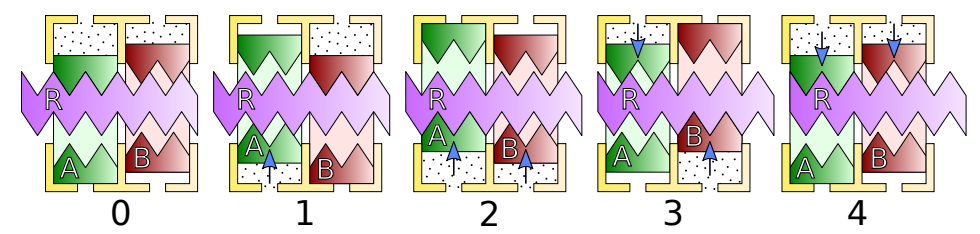

Figure 4.5: Five consecutive states of a single-speed linear stepper motor with the housing (yellow), rack (purple) and pistons (red and green).

\subsubsection{Production}

Figure 4.1 shows both assembled motors. The rigid parts of both motors were printed on a Stratasys Connex3 printer (Stratasys, Ltd., Eden Prairie, MN, USA) in VeroClear material, standard quality, glossy finish. The seals were laser-cut from $0.5 \mathrm{~mm}$ silicone rubber. The moving parts were lubricated with petroleum jelly (vaseline) and the housing was glued together using Loctite 406. Polyurethane tubing with an inner diameter of $2.5 \mathrm{~mm}$ and a length of $5 \mathrm{~m}$ was used to connect the motors to the valves. All used materials are non-metallic, non-magnetic and non-conductive.

\subsubsection{Position control}

The linear actuator can perform displacements of $0.3 \mathrm{~mm}, 1.4 \mathrm{~mm}, 1.7 \mathrm{~mm}$ or $2.0 \mathrm{~mm}$ in either direction within a single step. The greatest common divisor of the available step sizes is $0.1 \mathrm{~mm}$, which is the discretization error when 
executing an arbitrary number of steps.

Given a target displacement $d$, the number of large steps $m$ and small steps $n$ need to be calculated such that $1.7 m+0.3 n=d$. The range of motion of both sides gives an additional constraint and the total number of steps, $\max (|m|,|n|)$, is to be minimized for efficient operation. The lower bound for the required number of steps is $\left\lceil\frac{d}{2.0}\right\rceil$ and an upper bound is $\left\lceil\frac{d}{1.7}+20\right\rceil$. The optimal values for $m$ and $n$ can be found with an iterative algorithm or an exhaustive search over the whole state space.

For the rotational actuator the possible displacements in a single step are $2.86^{\circ}, 10^{\circ}, 12.86^{\circ}$ and $22.86^{\circ}$. The greatest common divisor is $1.43^{\circ}$ and the required number of steps to perform a target angular displacement can be found using the same methods as for the linear stepper motor.

The execution of large and small steps in opposite directions may cause overshooting, for example when a net displacement of $0.1 \mathrm{~mm}$ has to be executed. If overshoot is undesired, the precision of small adjustments changes to $0.3 \mathrm{~mm}$ which still complies with the sub-millimeter accuracy requirement. It is also possible to avoid overshooting in one particular direction only (e.g. the forward direction) by operating the motor sides in a certain sequence such that all backward motion(s) are performed prior to all forward motion(s). In that situation, any overshoot only occurs in the backward direction.

To reduce the hysteresis effect, setpoints for both motor sides should be consistently approached in forward direction. If a setpoint was approached in backward direction, the associated hysteresis effect can be cancelled by executing reciprocal steps.

\subsection{Evaluation}

To study the transient behaviour, positional accuracy, speed and maximum force/torque of the motors, several experiments were performed.

\subsubsection{Measurement setup}

Figure 4.6 shows the measurement setup for the linear and rotational motors. The linear motor is attached to a sliding rail of which the position is measured with an optical encoder of type EM2-0-1000-I (US Digital, Vancouver, WA, USA) with a resolution of $0.00635 \mathrm{~mm}$. Forces on the slider are generated by bodies with known mass hanging on a pulley.

The rotational motor drives a spindle with radius $15 \mathrm{~mm}$ to lift bodies of known mass. The angular displacement is measured using an optical encoder of type HEDS-9140 (Broadcom Ltd., San Jose, CA, USA) with a resolution of $0.18^{\circ}$. 


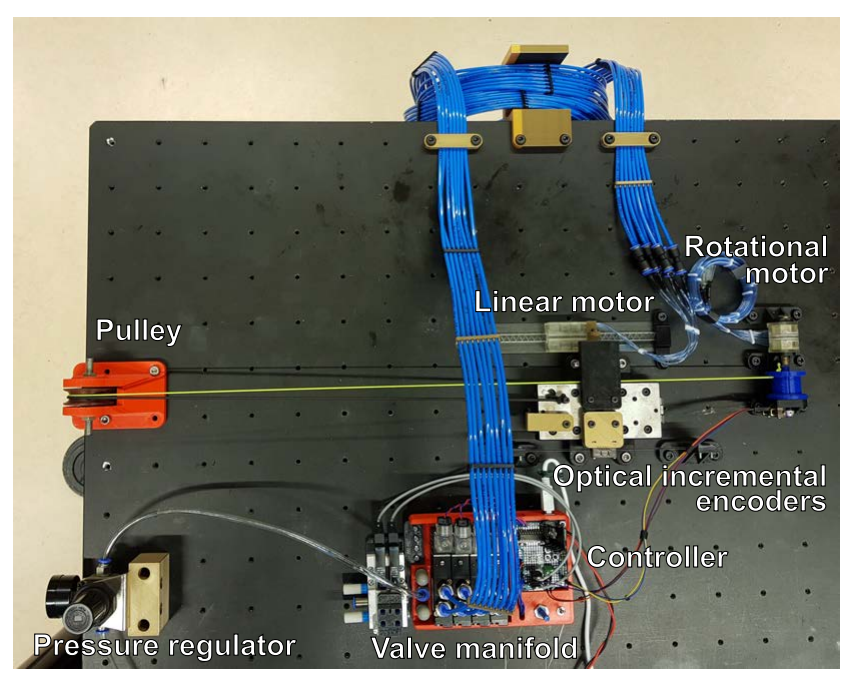

Figure 4.6: Measurement setup for linear and rotational motors. The motor's outputs are connected to weights of known mass hanging over a pulley. The pressure regulator can be adjusted to find the minimum pressure required for the motor to lift the given weights. Optical encoders track the precise displacements of the motors.

Polyurethane tubes (outer diameter $4 \mathrm{~mm}$, inner diameter $2.5 \mathrm{~mm}$, length $5 \mathrm{~m}$ ) connect either motor to the valves of type PV5211-24VDC-1/8. The system pressure is adjusted by a manual pressure regulator and observed by a pressure gauge.

An Arduino controls the valves at a fixed frequency of $10 \mathrm{~Hz}$ in all tests while keeping track of the optically-encoded position of the motor.

\subsubsection{Experiments}

\section{Positional accuracy}

In the positional accuracy experiments, the displacements of both motors were recorded while navigating through a sequence of setpoints. All positions were zero-calibrated at $\mathrm{t}=0 \mathrm{~s}$. The transient displacement was studied for overshoot and delay characteristics. The offset between setpoint and measured steadystate position resulted in a measure of positional accuracy.

\section{Force and torque}

The linear motor was loaded using bodies with a range of masses in steps of $0.5 \mathrm{~kg}$. For each load the lowest pressure was determined such that the largestep side of the motor was just able to lift the applied weight without skipping 


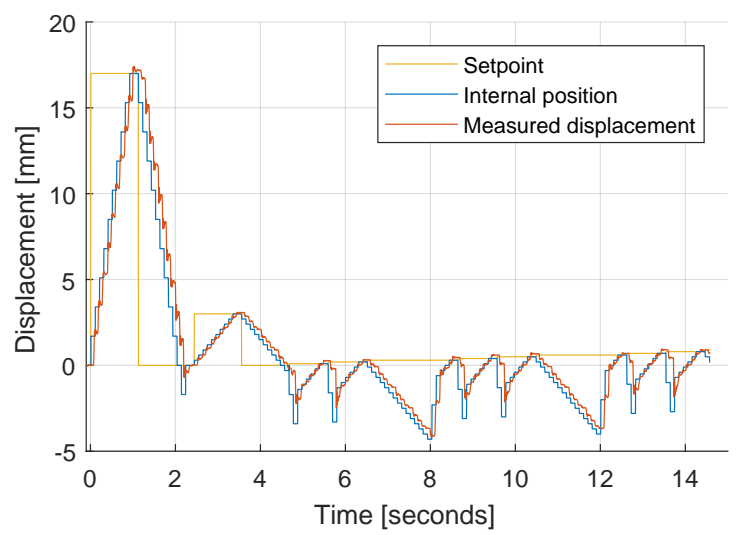

Figure 4.7: Transient displacement of linear motor. In the first two seconds the large-step side $(1.7 \mathrm{~mm})$ was operated, followed by operation of the small-step side $(0.3 \mathrm{~mm})$. Afterwards a series of net displacements of $0.1 \mathrm{~mm}$ were executed by driving combinations of large and small steps in such a way that no overshooting occurs in forward direction.

steps at a frequency of $10 \mathrm{~Hz}$. The same procedure was performed for the small-step side of the motor.

The rotational motor was tested using the same setup, but its spindle was loaded with a series of masses in steps of $0.1 \mathrm{~kg}$.

\subsubsection{Results}

\section{Transient displacement}

Figure 4.7 shows the transient displacement of the linear motor. From $\mathrm{t}=0 \mathrm{~s}$ it executes ten large steps, travelling $17 \mathrm{~mm}$ within one second. The average measured propagation delay was $70 \mathrm{~ms}$. In the next second it travels back to $\mathrm{x}=0 \mathrm{~mm}$. The deviation between internal and measured position is corrected by the extra reciprocating step at $\mathrm{t}=2.2 \mathrm{~s}$. From $\mathrm{t}=2 \mathrm{~s}$ it executes ten small steps to $\mathrm{x}=3.0 \mathrm{~mm}$ and back to the origin. From there on the setpoint is incremented in steps of $0.1 \mathrm{~mm}$ and it can be seen that for every $0.1 \mathrm{~mm}$ net displacement the motor executes a number of backward steps followed by a number of forward steps. Upon reaching the setpoint in forward direction the motor overshoots by less than $0.1 \mathrm{~mm}$ and the steady-state error is $0.1 \mathrm{~mm}$ on average. The recorded movements can also be viewed in the supplementary video.

Figure 4.8 shows the transient angular displacement of the rotational motor. The setpoint position is increased in steps of $2.86^{\circ}$ and it can be observed that in most cases four steps are required. The small-step side first executes two backward steps followed by one forward step after which the large-step side 


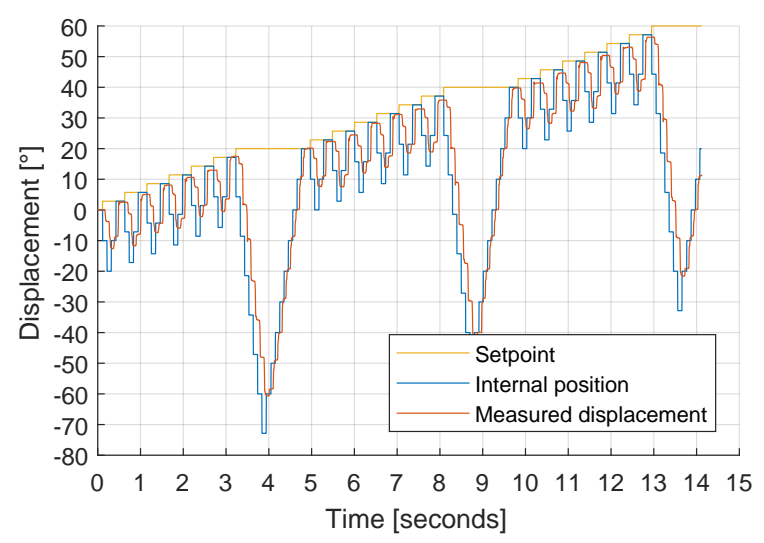

Figure 4.8: Transient displacement of rotational motor. A series of $2.86^{\circ}$ steps were executed by rotating the two sides in opposite directions. Certain additional steps were necessary to take hysteresis and range constraints into account.

executes one forward step. More steps are executed around $t=4 \mathrm{~s}$ and $\mathrm{t}=9 \mathrm{~s}$ to avoid wrapping the pneumatic tubes, hereby rotating at $100 \%$ s to $128 \%$ s. During forward motion of the motor sides, it follows the internal setpoint with a time delay of approximately $60 \mathrm{~ms}$. Upon reaching each setpoint the error between setpoint and observed position was measured to be $-0.69 \pm 0.52^{\circ}$.

\section{Force and torque}

Figure 4.9 shows the maximum force of both sides of the linear motor at different pressures. At a pressure of $0.3 \mathrm{MPa}$ the small-step side delivers $24 \mathrm{~N}$ which means an efficiency of $\frac{24}{100}=24 \%$, while the large-step rack exerts $29 \mathrm{~N}$ resulting in an efficiency of $\frac{29}{53}=55 \%$. Examination of the teeth shape under a microscope (Figure 4.11) revealed that the small-step teeth are rounded due to $3 \mathrm{D}$ printing effects and that the actual teeth depth is $1.2 \mathrm{~mm}$ instead the designed $2.0 \mathrm{~mm}$, reducing the effectiveness of the wedge mechanism. When engaged by the piston teeth, the effective wedge ratio at the contact point is $\frac{1}{\tan 26^{\circ}}=2.05$ and the difference with the theoretical value of 3.33 makes up for the majority of the difference in observed mechanical effiency between both sides of the linear motor.

In case of operating the motor under a $24 \mathrm{~N}$ load the maximum combined work per step is $24 \mathrm{~N} \times(0.3+1.7) \mathrm{mm}=48 \mathrm{~mJ}$. At an operating frequency of $10 \mathrm{~Hz}$ this results in a maximum power of $0.48 \mathrm{~W}$.

Figure 4.10 shows the maximum torques for the rotational motor. At a pressure of $0.3 \mathrm{MPa}$ the torque of the large-step side was measured to be $74 \mathrm{~N} \mathrm{~mm}$, resulting in a mechanical efficiency of $\frac{74}{167}=44 \%$. The small-step side is able 


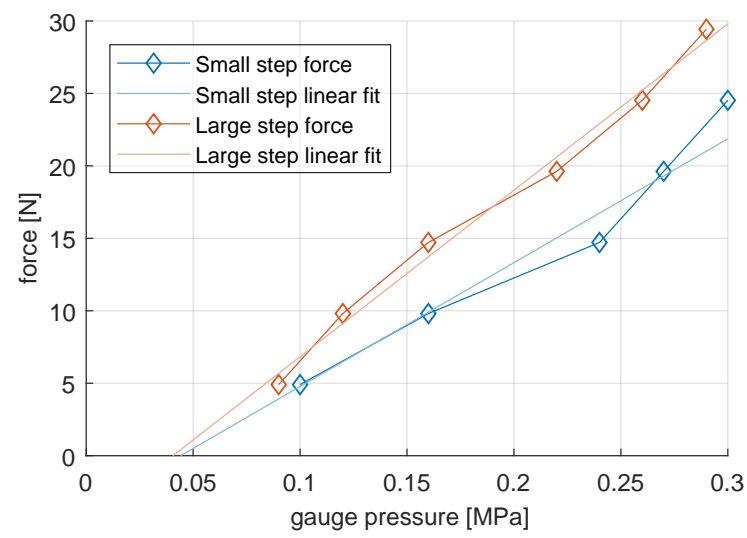

Figure 4.9: Maximum forces of the linear motor for a range of pressures, for both motor sides.

to exert the same torque at a pressure of $0.22 \mathrm{MPa}$, resulting in a mechanical efficiency of $\frac{74}{0.733 \times 214}=47 \%$.

The combined work per step under a $72 \mathrm{~N} \mathrm{~mm} \mathrm{load} \mathrm{is} 72 \mathrm{~N} \mathrm{~mm} \times(0.224+0.175) \mathrm{rad}=$ $29 \mathrm{~mJ}$. At a stepping frequency of $10 \mathrm{~Hz}$ this gives a maximum power of $0.29 \mathrm{~W}$.

\subsection{Discussion}

The linear motor has shown that it can move with a speed of $20 \mathrm{~mm} / \mathrm{s}$, has a minimum step size of $0.3 \mathrm{~mm}$, a positioning accuracy of $0.1 \mathrm{~mm}$ and a maximum force of $24 \mathrm{~N}$ at a pressure of $0.3 \mathrm{MPa}$. A displacement of $100 \mathrm{~mm}$ can be performed by executing 56 large and 16 small steps which takes $5.6 \mathrm{~s}$ in total. This is an order of magnitude faster than state-of-the-art stepper motors, without compromising on force or accuracy.

The rotational motor can rotate at a speed of up to $229^{\circ} / \mathrm{s}$, achieve an accuracy of approximately $1^{\circ}$, has a minimum step size of $2.86^{\circ}$ (when operating both sides in opposite direction) and delivers a maximum torque of $74 \mathrm{~N} \mathrm{~mm}$ at $0.3 \mathrm{MPa}$. A rack-and-pinion with radius $5 \mathrm{~mm}$ theoretically converts these figures to a linear speed of up to $20 \mathrm{~mm} / \mathrm{s}$ with an accuracy of $0.09 \mathrm{~mm}$ and a maximum force of $15 \mathrm{~N}$.

The maximum forces and torques are limited by system pressure, cylinder cross-sectional area and the effective wedge ratio of the mechanical force transfer from piston to rack or gear. A larger cylinder cross-sectional area would result in enlarged overall dimensions, while increased pressure or a higher wedge ratio require the use of stronger materials to withstand the increased material 


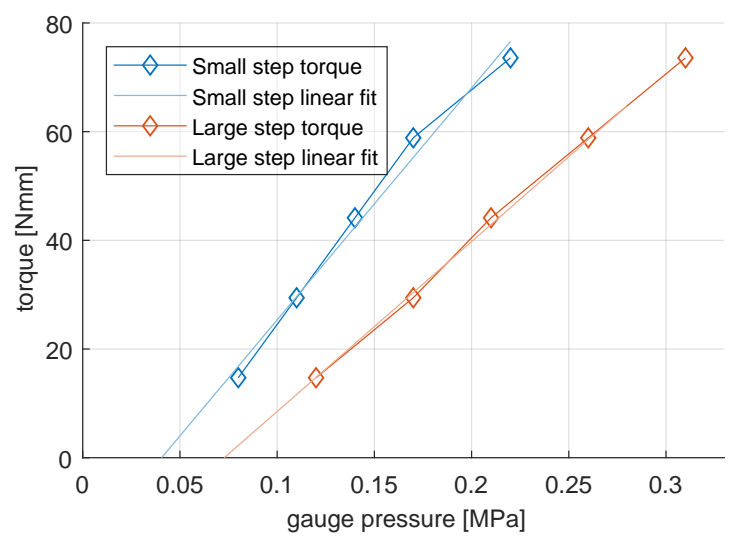

Figure 4.10: Maximum torques of rotational motor for a range of pressures, for both motor sides.

stresses. In the linear motor the force is also limited by the roundedness of the teeth in the small-step rack due to manufacturing limitations, resulting in a suboptimal wedge mechanism.

In the rotational motor the small-step side requires $30 \%$ lower pressure to deliver the same amount of torque than the large-step side. The reason is that the step angle is different while the teeth depth is equal, resulting in inconsistent wedge ratios. A performance gain can be expected by better balancing the maximum torques of both sides, which could be achieved by adjusting the dimensions of the teeth or cylinders while taking the size and material strength constraints into account.

The range of motion of the linear actuator depends on the lengths of the large-step and small-step racks. The large-step rack must be long enough to cover the entire workspace, while the small-step rack only needs sufficient freedom to make the required small-step adjustements. The small-step side can move continuously in the rotational motor while the large-step side is constrained by the available space to wrap and un-wrap the pneumatic tubes.

The bandwidth is limited to $10 \mathrm{~Hz}$, so higher stepping frequencies result in reduced output forces. Steps may be skipped when load forces exceed the motor output forces and these skipped steps cannot be immediately detected without position feedback. While it is possible to incorporate MR safe position feedback with optical sensors, a possibly more viable approach to further increase travel speed is to add a third stage resulting in a triple-speed pneumatic stepper motor.

Eight pneumatic tubes with outer diameter $3 \mathrm{~mm}$ to $4 \mathrm{~mm}$ are needed to connect one dual-speed motor to four valves of the controller, resulting in a rel- 


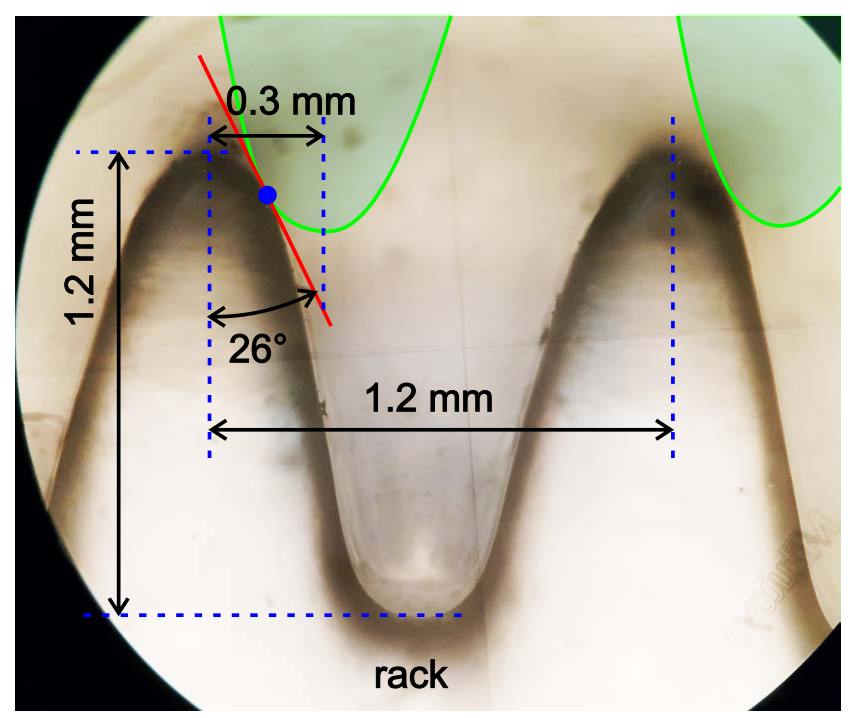

Figure 4.11: Microscopic picture of the small-step rack showing the roundedness of its teeth. One point of contact with the engaging piston teeth (sketched green) is shown as a blue dot and its tangent line in red. The slope of this tangent line determines the effective wedge ratio of the mechanical transfer from piston to rack.

Table 4.1: Average movement time of biopsy robots

\begin{tabular}{c|c} 
Robotic system & Operating time (minutes) \\
\hline Stoianovici et al. (MrBot) [120] & $1: 36 \pm 1: 24$ \\
Bomers et al. (RCM) [15] & $5: 48$ \\
Groenhuis et al. (Stormram 4) [55] & $1: 30$ \\
System using dual-speed linear motors & $0: 11$ (projected)
\end{tabular}

atively large cable bundle and complex controller for the whole robotic system. The number of tubes may be reduced by employing cylinder sharing techniques, at the cost of a reduced state space.

The size, force, accuracy and speed characteristics of both dual-speed stepper motors make these suitable for effective actuation of MR safe robotic systems. In particular, the linear motor could be used in the linear stages of the Stormram 4 robot [55]. Based on the modified joint velocities the projected average time required to move the upgraded robot towards a new target location can be calculated. A typical movement involves a displacement over $50 \mathrm{~mm}$ (28 steps of $1.7 \mathrm{~mm}$ plus 8 steps of $0.3 \mathrm{~mm}$ ) followed by an angulation of $10^{\circ}$ (40 steps of $0.25^{\circ}$ ) and the insertion of a biopsy needle over a distance of $70 \mathrm{~mm}$ (41 steps of $1.7 \mathrm{~mm}$ plus one of $0.3 \mathrm{~mm}$ ). In total this requires 109 steps which 
can be executed in $10.9 \mathrm{~s}$ at a stepping frequency of $10 \mathrm{~Hz}$. For comparison, Table 4.1 lists the average movement durations for different MR safe biopsy robots driven by pneumatic stepper motors.

\subsection{Conclusion}

Two space-efficient dual-speed motors have been developed. Both of them are one order of magnitude faster than state-of-art pneumatic stepper motors, without compromising on accuracy or force. Limitations include the high number of pneumatic tubes and the additional complexity involved in manufacturing and control, especially when enhanced accuracy without overshooting is desired. The average required time to manipulate the robot using such motors to a given position can be reduced from minutes to approximately ten seconds. This improves the potential for MR safe robotic devices to improve accuracy and reduce the procedure time of a MR guided breast biopsy.

\section{Acknowledgements}

The authors thank Gerben te Riet o/g Scholten for the support in the production process of the motors. 


\section{Miniaturization of MR Safe Pneumatic Rotational Stepper Motors}

V. Groenhuis, F. J. Siepel, and S. Stramigioli. Miniaturization of MR Safe Pneumatic Rotational Stepper Motors. In IEEE/RSJ International Conference on Intelligent Robots and Systems, Macau, 2019. IEEE 


\section{$5.1 \quad$ Abstract}

Pneumatic rotational stepper motors can be used to actuate MR (magnetic resonance) safe robotic systems. This paper describes novel techniques to minimize the volumetric size and/or step size of such motors in order to cope with the limited space requirements while still delivering high precision. Three designs are presented: the R-10 measures $1.0 \mathrm{~cm}^{3}$, has step size $12.9^{\circ}$ and torque $1.2 \mathrm{~N} \mathrm{~mm}$. The R-40 measures $25.6 \mathrm{~cm}^{3}$, has step size $1.01^{\circ}$ and torque $470 \mathrm{Nmm}$. The R-54 measures $46.7 \mathrm{~cm}^{3}$, has step size $1.01 \mathrm{~m}^{\circ}$ and torque $240 \mathrm{Nmm}$. The particularly small step size in the R-54 motor is achieved by using a high-reduction planetary gear.

These three motors demonstrate that small-scale rotational stepper motors with a wide range of step sizes and good torque characteristics can be constructed that surpass state-of-art designs by a considerable margin. This allows the advancement of MR safe robotics towards more compact and versatile designs, and also overcome certain existing limitations by combining multiple motors with different specifications.

\subsection{Introduction}

The MRI scanner offers high sensitivity compared to other three-dimensional imaging modalities without the adverse effects of ionizing radiation as in CT. Example applications are in periodic breast screening of high-risk patients, in addition to mammography and ultrasonography. When a suspicious lesion is found then it may need to be biopsied. If this lesion is only visible on MRI then an MRI-guided biopsy procedure is needed. Current manual procedures have drawbacks in terms of accuracy and speed [129], which have led to the research in MR safe and MR conditional robotic systems for breast biopsy.

MR safe robotic systems are highly preferred over MR conditional systems as the former utilizes the full potential of high-field MRI scanners with no restrictions. A major challenge in the development of any MR safe robotic system is the design of the actuator which needs to be free of metallic, ferromagnetic and conductive materials. The actuators must also be small enough to make the surgical robot fit inside the MRI scanner alongside the patient. With electromagnetic motors ruled out, pneumatics is one option which offers certain advantages over hydraulics, such as the small size and acceptability of small leakages [28, 92]. State-of-art pneumatic stepper motors have relative large dimensions (several centimetres) and/or step sizes (several degrees), so there is a need for smaller designs to actuate small-scale, high-precision MR safe robotic systems. 


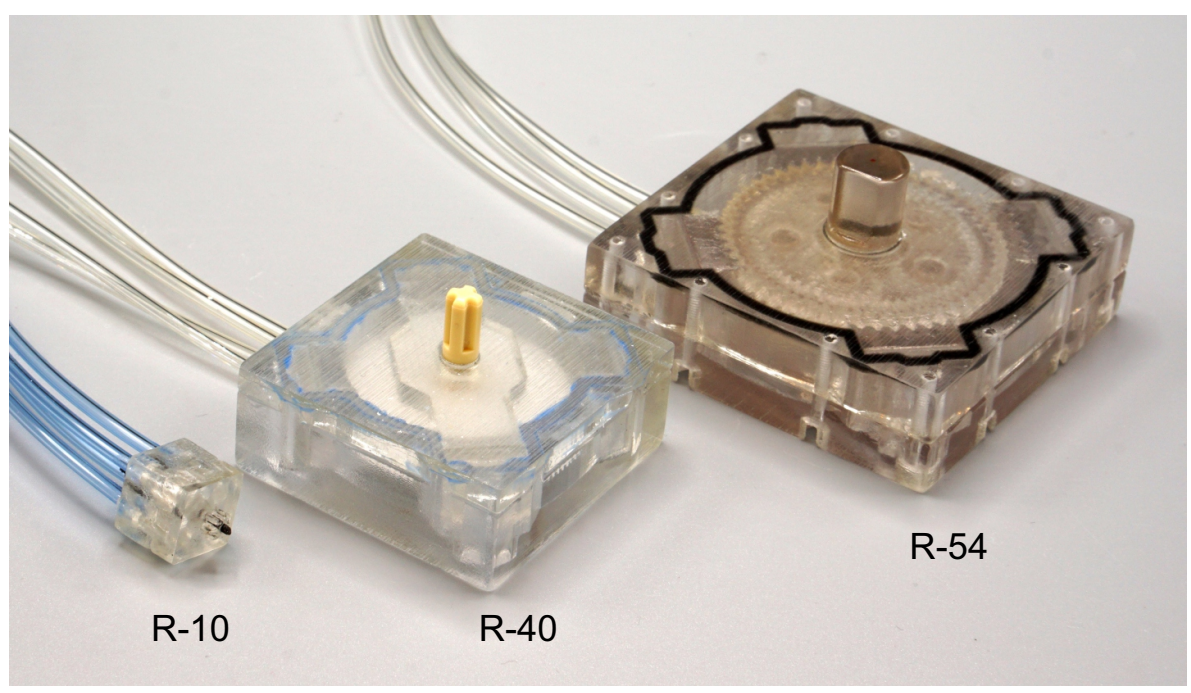

Figure 5.1: The R-10, R-40 and R-54 pneumatic stepper motors.

\subsubsection{State of Art}

Several types of MR safe pneumatic stepper motors have been developed with a broad range of specifications. Stoianovici et al. developed the PneuStep with a build volume of $200 \mathrm{~cm}^{3}$, a step size of $3.3^{\circ}$ and a maximum specified torque of $600 \mathrm{~N} \mathrm{~mm} \mathrm{[118].} \mathrm{Guo} \mathrm{et} \mathrm{al.} \mathrm{developed} \mathrm{a} \mathrm{pneumatic} \mathrm{stepper} \mathrm{motor} \mathrm{with} \mathrm{volume}$ $84.7 \mathrm{~cm}^{3}$, step size $60^{\circ}$ and torque $4.9 \mathrm{~N} \mathrm{~mm} \mathrm{[60].} \mathrm{Sajima} \mathrm{et} \mathrm{al.} \mathrm{developed} \mathrm{a}$ stepper motor with volume $28 \mathrm{~cm}^{3}$, step size $4.3^{\circ}$ and torque $170 \mathrm{~N} \mathrm{~mm} \mathrm{[109].}$ Farimani et al. developed the PneuAct with build size approx. $75 \mathrm{~cm}^{3}$ (including 1:40 gear), step size $3^{\circ}$ and torque $120 \mathrm{Nmm}$; a smaller version (without reduction gear) with build volume $4.6 \mathrm{~cm}^{3}$ and step size $120^{\circ}$ was also demonstrated [38]. Chen et al. developed a unidirectional stepper motor with build volume $4.7 \mathrm{~cm}^{3}$, step size $60^{\circ}$ and torque $2.4 \mathrm{~N} \mathrm{~mm} \mathrm{[27].}$

The authors of this paper previously presented several rotational stepper motors. The R-25 has a volume of $12.5 \mathrm{~cm}^{3}$, a step size of $6.9^{\circ}$ and a torque of $100 \mathrm{Nmm}$ [47]. A dual-speed version, the DR-32, was also demonstrated with a build volume of $22.6 \mathrm{~cm}^{3}$, differential step size $2.9^{\circ}$ and torque $74 \mathrm{~N} \mathrm{~mm} \mathrm{[52].}$

MR safe surgical robots powered by pneumatic stepper motors have also been demonstrated. The Sunram 5 and previous iterations from the authors of this paper are driven by combinations of linear and curved stepper motors $[54,55]$, while pneumatic robotic systems by other authors are typically driven by rotational or helical stepper motors [15, 121].

Fig. 5.9 graphically shows the build volumes and step sizes of the state-of- 


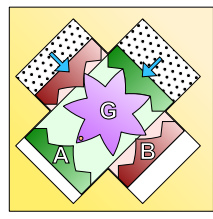

0

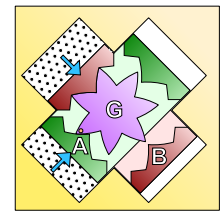

1

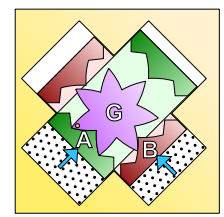

2

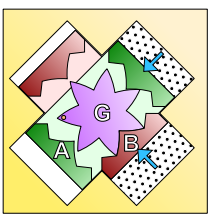

3

Figure 5.2: Sequence of piston states during clockwise rotation of the gear. After four steps the motor is back in the first state, but with the gear displaced by one tooth.

art motors in blue. It shows that these all have build volumes over $4 \mathrm{~cm}^{3}$ and step sizes higher than $2^{\circ}$. The smallest motors (in volume) are not directly usable due to the unidirectional mechanism (Chen) or lack of torque (Farimani). Bidirectional stepper motors with sufficient torque have relatively large dimensions, step sizes and/or a complicated design which limit the applicability in small-scale MR safe robotics. There is a need for new designs that have smaller dimensions and/or step sizes than state-of-art designs, hereby advancing the Pareto frontier in Fig. 5.9 towards the left and bottom edges of the graph.

This paper is organized as follows. First the working principles, design and construction of the R-10, R-40 and R-54 are described. Next, torque measurements and step size validation experiments are described alongside with the results. Finally, conclusions are drawn with an outlook of application in future MR safe robotic systems.

\subsection{Materials and Methods}

Three different rotational stepper motors are presented. All three models make use of two pneumatic cylinders with rectangular cross-section, positioned in a cross-shape that interact with a gear. Several aspects of this type of pneumatic stepper motor have been extensively described and investigated by Groenhuis et al. [47]. The state sequence is shown in Fig. 5.2: by applying pressure in the four chambers in the given sequence a step-wise rotational motion is generated.

The R-10 is optimized for minimal volume by making the cylinders and gears as small as practically feasible by rapid prototyping without taking step size or torque into account. In contrast, the R-54 is optimized for minimal step size by integrating a high-reduction planetary gear transmission. Finally, the R-40 is designed for minimal volume while keeping the step size fixed at approximately $1.0^{\circ}$. 


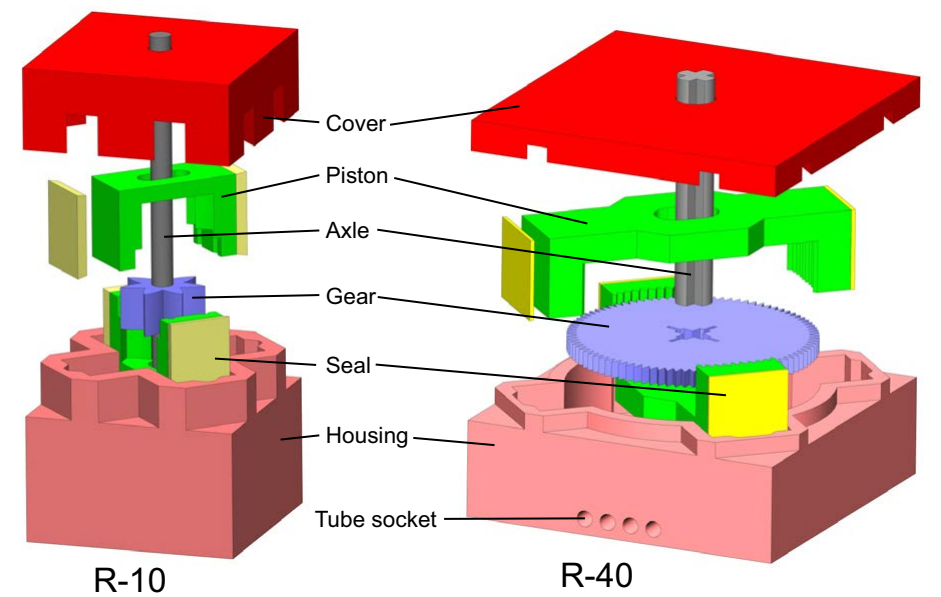

Figure 5.3: R-10 (left) and R-40 (right) CAD drawings, showing the housing with cover, axle, gear, two pistons and four seals. In the R-10 the pneumatic tube sockets are on the bottom of the housing and not visible. The R-40 has different dimensions and a higher number of teeth than the R-10.

\subsubsection{R-10 design}

The R-10 is a scaled-down version of the R-25. It consists of the housing with cover, two pistons with four silicone seals, one gear with seven teeth and the axle. The dimensions of the housing are $(10 \times 10 \times 10) \mathrm{mm}^{3}=1.0 \mathrm{~cm}^{3}$. Fig. 5.3(left) shows a CAD drawing of the motor.

The cylinder cross-sectional area measures $(3 \mathrm{x} 4) \mathrm{mm}^{2}=12 \mathrm{~mm}^{2}$. The gear has an outside diameter of $4 \mathrm{~mm}$, the seven teeth have a depth of $1 \mathrm{~mm}$. The step size is $\frac{90}{7}=12.9^{\circ}=0.224 \mathrm{rad}$. At a system pressure of $0.1 \mathrm{MPa}$ (approx. 1 bar) the piston force is $12 \cdot 10^{-6} \mathrm{~m}^{2} \cdot 0.1 \cdot 10^{6} \mathrm{~N} / \mathrm{m}^{2}=1.2 \mathrm{~N}$. The nominal work per step is $\frac{1}{2} \cdot 1 \mathrm{~mm} \cdot 1.2 \mathrm{~N}=0.6 \mathrm{~mJ}$, resulting in a nominal torque of $\frac{0.6 \mathrm{~mJ}}{0.224 \mathrm{rad}}=2.68 \mathrm{Nmm}$ for every $0.1 \mathrm{MPa}$ of pressure, equivalent to $26.8 \mathrm{Nmm} / \mathrm{MPa}$.

\subsubsection{R-40 design}

The R-40 is also based on the R-25 design and consists of the same type of components as the R-10. The main difference is that the gear has 89 teeth, resulting in a step size of $\frac{90}{89}=1.0112^{\circ}$. Given the minimum teeth tip spacing of $1.0 \mathrm{~mm}$ that can still be rapid prototyped without compromising on torque and reliability, the 89-teeth gear was designed to have an outside diameter of $30 \mathrm{~mm}$ and a teeth depth of $1.2 \mathrm{~mm}$. The external dimensions are $(40 \mathrm{x} 40 \mathrm{x}$ 16) $\mathrm{mm}^{3}=25.6 \mathrm{~cm}^{3}$. Fig. 5.3(right) shows a CAD drawing of the R-40 motor. 

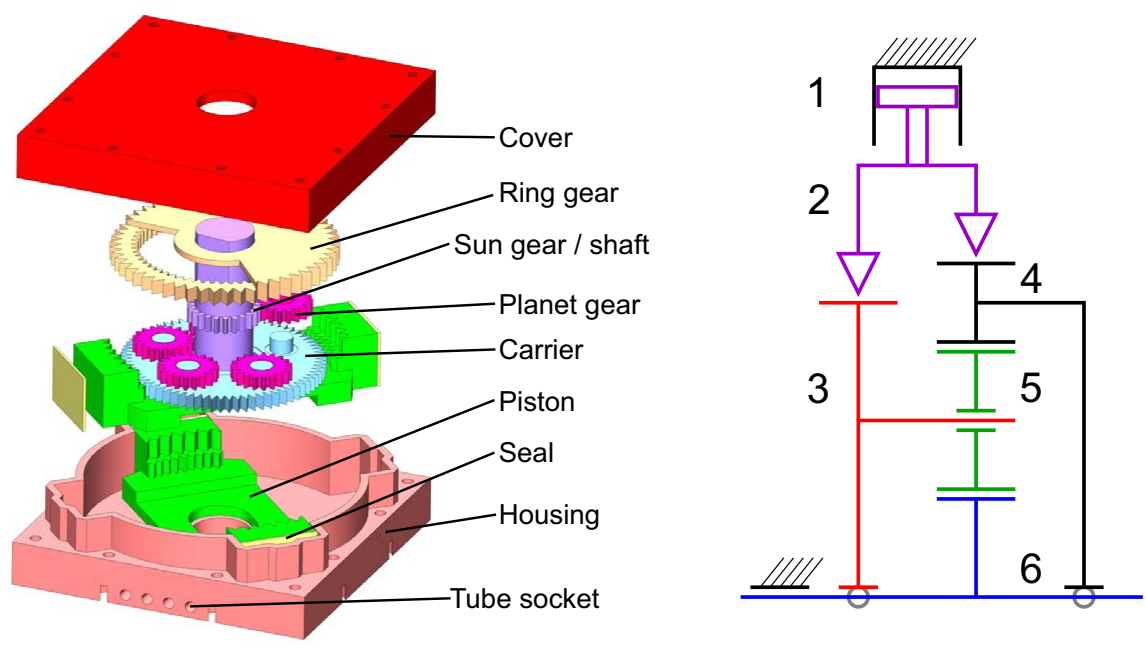

Figure 5.4: Left: R-54 CAD drawing. The housing and pneumatics are similar to that of the R-10 and R-40, but the single gear is replaced by a planetary gear mechanism consisting of the ring gear, sun gear, carrier and planet gears. The two pistons engage on both the carrier gear and the ring gear. Right: Kinematic diagram of the R-54 showing the pneumatic cylinder (1), piston with teeth (2), carrier (3), ring gear (4), planet gear (5) and sun gear on output shaft $(6)$.

The cylinders of the R-40 have a cross-sectional area of $(10 \times 10) \mathrm{mm}^{2}$. Combined with an effective stroke of $0.6 \mathrm{~mm}$, for every $0.1 \mathrm{MPa}$ of pressure this results in $100 \cdot 10^{-6} \mathrm{~m}^{2} \cdot 0.1 \cdot 10^{6} \mathrm{~N} / \mathrm{m}^{2} \cdot 0.6 \mathrm{~mm}=6.0 \mathrm{~mJ}$ of energy delivered per step. With a step angle of $1.0112^{\circ}=0.0176 \mathrm{rad}$ this corresponds to a theoretical torque of $\frac{6.0 \mathrm{~mJ}}{0.0176 \mathrm{rad}}=341 \mathrm{Nmm}$ for every $0.1 \mathrm{MPa}$ of pressure, equivalent to $3.41 \mathrm{Nm} / \mathrm{MPa}$.

\subsubsection{R-54 design}

The R-54 is different from the other two described motors in the sense that it includes a planetary gear, allowing for a significant reduction in step size. Fig. 5.4(left) shows a CAD drawing of the R-54 motor.

The kinematic diagram is shown in Fig. 5.4(right). The pneumatic cylinders (1) are attached to the housing and operate the pistons (2). The different teeth on the pistons engage with both the carrier (3) and the ring gear (4), resulting in step motions over different angles. The shafts of the planet gears (5) are mounted on the carrier (3) while the planet gears themselves are driven by the ring gear (4). Finally, the sun gear (6) is driven by the planet gears (5) and attached to the output shaft. 
The step sizes of the different bodies depend on te number of teeth on its perimeters. Let $T_{s}, T_{r}$ and $T_{p}$ be the teeth count of the sun, internal ring and planet gear, respectively. Likewise, let $T_{e}$ and $T_{c}$ be the teeth count of the (pneumatically actuated) external ring and carrier gears. Finally, let $S_{s}$, $S_{r}$ and $S_{c}$ be the step sizes (in degrees) of the sun, ring and carrier gears, respectively.

The goal is to find a suitable assignment of $\left(T_{s}, T_{p}, T_{r}, T_{c}, T_{e}\right)$ such that $S_{s}$ is minimal. For this we have to describe $S_{s}$ as function of the teeth parameters.

First, we calculate the step sizes $S_{r}$ and $S_{c}$ of the pneumatically actuated ring and carrier gears. As shown in Fig. 5.2, four steps advance the gear by exactly one teeth:

$$
S_{r}=\frac{360}{4 T_{e}}=\frac{90}{T_{e}}, S_{c}=\frac{90}{T_{c}}
$$

Next, we apply the planetary gear equations. The planet gears are constrained by the sun gear and internal ring gear:

$$
\begin{aligned}
T_{r} & =T_{s}+2 T_{p} \\
S_{s} & =-\frac{T_{r}}{T_{s}}\left(S_{r}-S_{c}\right)+S_{c}
\end{aligned}
$$

We use the following parameter constraints to take account for rapid prototyping ability, proper gear meshing and keeping dimensions sufficiently small:

$$
\begin{aligned}
16 & \leq T_{s}, T_{p} \leq 25 \\
T_{e}, T_{c} & \leq 81 \\
T_{e}, T_{c} & \equiv 1 \quad(\bmod 4)
\end{aligned}
$$

The latter constraint simplifies piston design as the piston teeth of all four sides can be shaped equally.

The free parameters are $T_{s}, T_{p}, T_{e}$ and $T_{c}$ and the optimization criterion is $\min \left|S_{s}\right|$. The state space size is $10^{2} \cdot 20^{2}=40000$, which is small enough to perform an exhaustive search over all possible parameter combinations. The optimum has the following values:

$$
\left(T_{s}, T_{p}, T_{r}, T_{c}, T_{e}\right)=(23,19,61,73,53)
$$

Filling these values in Eq. 5.3 gives: 


$$
\begin{aligned}
S_{s} & =-\frac{T_{r}}{T_{s}}\left(\frac{90}{T_{e}}-\frac{90}{T_{c}}\right)+\frac{90}{T_{c}} \\
& =90 \cdot \frac{T_{s} T_{e}+T_{r} T_{e}-T_{r} T_{c}}{T_{s} T_{e} T_{c}} \\
& =90 \cdot \frac{23 \cdot 53+61 \cdot 53-61 \cdot 73}{23 \cdot 53 \cdot 73} \\
& =90 \cdot \frac{-1}{88987}=-0.0010114^{\circ}=-1.0114 \mathrm{~m}^{\circ}
\end{aligned}
$$

For the planetary gears we use gear modulus of 0.5 which corresponds to a teeth spacing of $\frac{\pi}{2}=1.6 \mathrm{~mm}$. The outer diameter of the external ring gear is set to $39 \mathrm{~mm}$ with a teeth depth of $2 \mathrm{~mm}$ to keep sufficient margin (approx. $1.5 \mathrm{~mm}$ ) to the internal ring gear. The carriage gear has more teeth $\left(T_{c}=73\right)$ than the external ring gear $\left(T_{e}=53\right)$, but the teeth spacing is still large enough to allow a slightly smaller outer diameter of $35 \mathrm{~mm}$. This smaller diameter makes it easier to construct the piston teeth by stacking different rows of teeth without need for supports.

The pneumatic cylinders have a cross-sectional area of $(14 \times 10) \mathrm{mm}^{2}=140 \mathrm{~mm}^{2}$, giving $14 \mathrm{~N}$ of force per $0.1 \mathrm{MPa}$ of pressure. The teeth depth is $2 \mathrm{~mm}$, so every $0.1 \mathrm{MPa}$ of pressure results in $14 \mathrm{~N} \cdot \frac{1}{2} \cdot 2 \mathrm{~mm}=$ $14 \mathrm{~mJ}$ of energy delivered per step.

There are two parallel drivetrains from the pneumatic cylinders to the output shaft. As the carrier and ring gears are not coupled together, overloading either of them will result in skipped steps. It is therefore required to evaluate each drivetrain separately in determining the theoretical torque on the output shaft.

- Ring gear: The step size is $\frac{90}{53} \approx 1.70^{\circ} \approx 0.030 \mathrm{rad}$ and this corresponds to a theoretical torque on the ring of $\frac{14 \mathrm{~mJ}}{0.030 \mathrm{rad}} \approx 472 \mathrm{~N} \mathrm{~mm}$ for every $0.1 \mathrm{MPa}$ of pressure, equivalent to $4.72 \mathrm{Nm} / \mathrm{MPa}$. The torque ratio from ring to sun is $\left|-\frac{T_{s}}{T_{r}}\right|=\frac{23}{61} \approx 0.38$, so the theoretical output torque (through the ring) is $\frac{23}{61} \cdot 4.72 \mathrm{Nm} / \mathrm{MPa} \approx 1.78 \mathrm{Nm} / \mathrm{MPa}$.

- Carrier gear: The step size is $\frac{90}{73} \approx 1.23^{\circ} \approx 0.022 \mathrm{rad}$ and this corresponds to a theoretical torque on the carrier of $\frac{14 \mathrm{~mJ}}{0.022 \mathrm{rad}} \approx 651 \mathrm{Nmm}$ for every $0.1 \mathrm{MPa}$ of pressure, equivalent to $6.51 \mathrm{Nm} / \mathrm{MPa}$. The torque ratio from carrier to sun is $\left|\frac{1}{1+T_{r} / T_{s}}\right|=\frac{T_{s}}{T_{r}+T_{s}}=\frac{23}{84} \approx 0.27$, so the theoretical output torque (through the ring) is $\frac{23}{84} \cdot 6.51 \mathrm{Nm} / \mathrm{MPa} \approx 1.78 \mathrm{Nm} / \mathrm{MPa}$.

The theoretical output torques for both drivetrains are equal (up to a rounding error), which is the consequence of having almost equal step sizes in opposite directions and equal teeth depth in the carrier gear and external ring gear. 
The small difference between the step sizes account for the $1.01 \mathrm{~m}^{\circ}$ net step size. When the output shaft is loaded by some torque, this torque propagated through both drivetrains independent of the torque direction and rotational motion. As the transferred output torque through both drivetrains is approximately equal, we can define the theoretical output torque of the R-54 motor as $1.78 \mathrm{Nm} / \mathrm{MPa}$.

\subsubsection{Fabrication}

The majority of the parts was 3-D printed on an Objet Connex3 (Stratasys, Eden Prairie, MN, USA) in VeroClear material in standard quality, glossy surface mode. The housing cover in the R-54 also includes an integrated stationary seal printed in TangoBlack. The gears in the R-10 and R-40 motors were lasercut from $2 \mathrm{~mm}$ acetal. The axle in the R-10 was cut from a $1 \mathrm{~mm}$ diameter carbon fibre rod and the axle in the R-40 consists of a Lego Technic axle. The moving seals were laser-cut from a $0.5 \mathrm{~mm}$ thick silicone rubber sheet with an approximate stiffness of Shore A 60.

After assembling the motor parts the cover was glued on the housing using cyanoacrylate (Loctite $406+770$ ). This glue was also used to fix the four pneumatic tubes ( $2 \mathrm{~mm}$ outer diameter) in the sockets and to fix the gear in the R-10 to the axle. The assembled motors are shown in Fig. 5.1.

\subsubsection{MR safety analysis}

The three motors consist of the following materials:

- VeroClear: all motors (housing, pistons, planetary gears)

- TangoBlack: R-54 (stationary seal)

- Acetal (POM): R-10 and R-40 (gears)

- Carbon fibre: R-10 (axle)

- ABS: R-40 (axle)

- Silicone rubber: all motors (moving seals)

- Polyurethane: all motors (tubing)

- Glue (Loctite 406, 770): all motors

- Sealant (Loctite 5926): R-40 


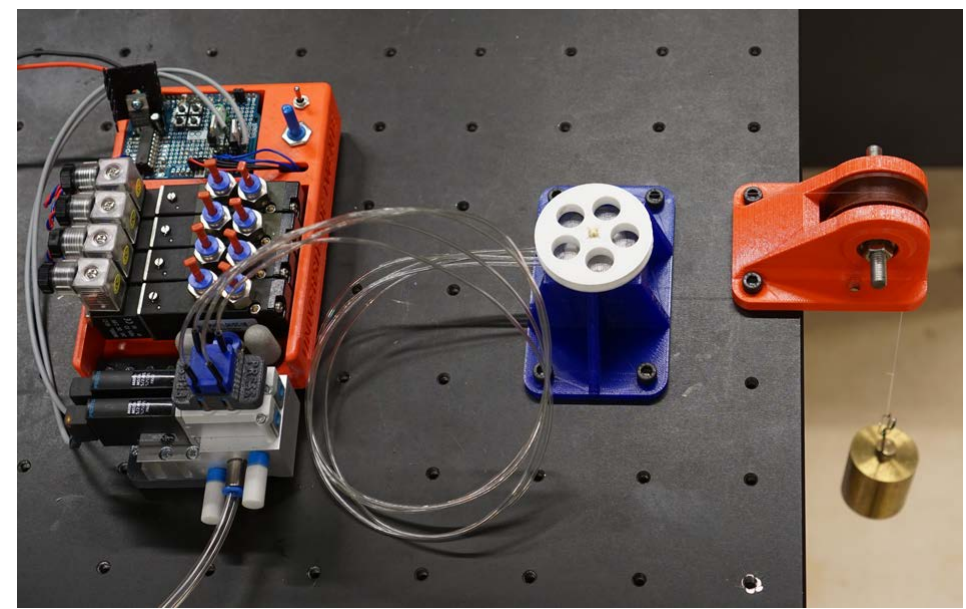

Figure 5.5: Setup for torque measurements. The valves are controlled by an Arduino controller, sending pressure waveforms to the motor through the four tubes. The motor then lifts a weight of known mass over a pulley by means of a winch.

All listed materials are non-metallic, non-ferromagnetic and non-conductive, with one exception: carbon fibre is conductive. This implies that only the R-40 and R-54 are classified MR safe according to the ASTM F2503 standard. The R-10 may be classified MR safe when the carbon fibre axle is replaced by a different material.

\subsection{Experimental Results and Analysis}

The three motors were characterized by measuring the torque-pressure relationship. Also, the actual step sizes were validated by observation.

\subsubsection{Measurement setup}

For torque measurements the setup shown in Fig. 5.5 is used. The motor is mounted on the table with a frame and a winch is placed on the motor shaft. This winches a fishing line over a pulley, lifting up one or more weights of known mass. The radius of the winch is $3.5 \mathrm{~mm}$ for the R-10 motor and $24 \mathrm{~mm}$ for the R-40 and R-54 motors. The total mass is varied and for each given mass a measurement point is collected. The measurement involves slowly increasing the system pressure using a pressure regulator until the motor can consistently lift the given mass. This procedure is repeated to obtain at least five data points. The data points for each motor are then plotted in a pressure-torque graph. 


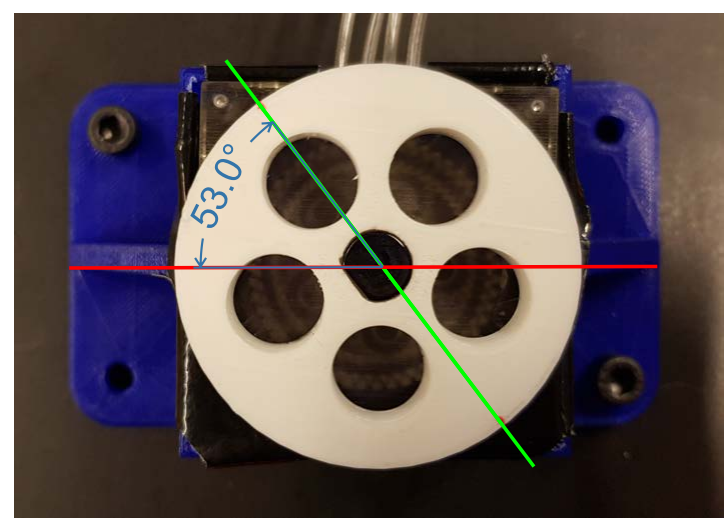

Figure 5.6: Setup for angle measurements on the R-54 motor.

The step size validation involves counting the number of steps in one full revolution and comparing these to the calculated values. For the R-10 the theoretical number of steps is 28 , for the R-40 it is 356 steps and for the R-54 it is 355948 steps. Validating these numbers are trivial for the R-10 and R40. For the R-54, validation is done by running the motor at a fixed stepping frequency of $99 \mathrm{~Hz}$ and observing the angle of the output shaft pulley at different moments using a camera. The observed angular velocity can then be compared to the predicted angular velocity as calculated from the step angle and stepping frequency.

\subsubsection{Measurement results}

\section{Torque measurement results}

Fig. 5.7 shows the torque graph of the R-10, R- 40 and R-54 motors. The maximum torque of the R-10 was measured to be $1.2 \mathrm{Nmm}$ at a pressure of $0.4 \mathrm{MPa}$. The graph is approximately linear, with a slope of $3.81 \cdot 10^{-9}$ $\mathrm{NPa}^{-1}$ or $3.81 \mathrm{Nmm} / \mathrm{MPa}$. When compared to the theoretical torque of $26.8 \mathrm{Nmm} / \mathrm{MPa}$, the mechanical efficiency is $\frac{3.81}{26.8}=14 \%$.

The maximum torque of the R-40 was measured to be $470 \mathrm{~N} \mathrm{~mm}$ at a pressure of $0.26 \mathrm{MPa}$. This graph is also approximately linear, with a slope of $2.03 \mathrm{Nm} / \mathrm{MPa}$. When compared to the theoretical torque of $3.41 \mathrm{Nm} / \mathrm{MPa}$, the mechanical efficiency is $\frac{2.03}{3.41}=60 \%$.

The R-54 was measured to have a maximum torque of $240 \mathrm{~N} \mathrm{~mm}$ at a pressure of $0.24 \mathrm{MPa}$. Again, this graph is approximately linear and the slope is $1.05 \mathrm{Nm} / \mathrm{MPa}$. When compared to the theoretical torque of $1.78 \mathrm{Nm} / \mathrm{MPa}$, the mechanical efficiency is $\frac{1.05}{1.78}=59 \%$. 


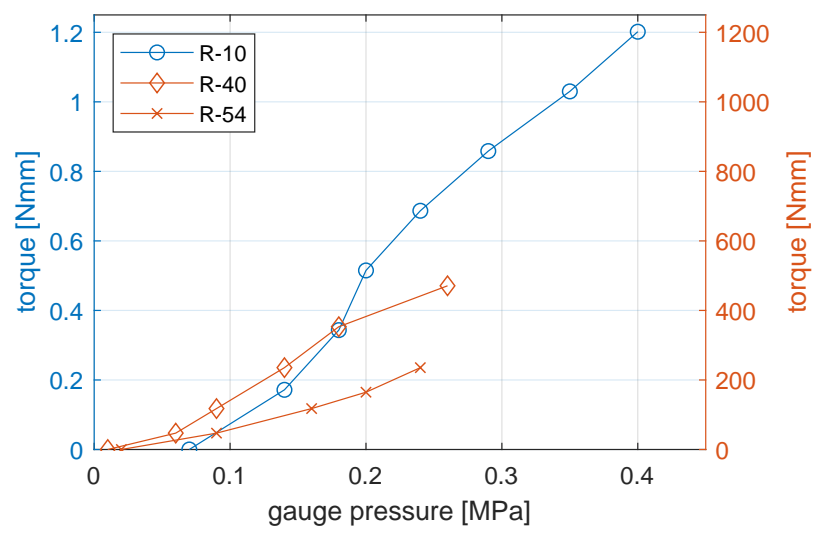

Figure 5.7: Torque versus pressure diagrams for the three motors.

Table 5.1: Comparison of R-10, R-40 and R-54 pneumatic stepper motors.

\begin{tabular}{c|l|l|l} 
Motor & R-10 & R-40 & R-54 \\
\hline Dimensions & $10 \times 10 \times 10 \mathrm{~mm}^{3}$ & $40 \times 40 \times 16 \mathrm{~mm}^{3}$ & $54 \times 54 \times 16 \mathrm{~mm}^{3}$ \\
& $=1.0 \mathrm{~cm}^{3}$ & $=26 \mathrm{~cm}^{3}$ & $=47 \mathrm{~cm}^{3}$ \\
Step size & $12.9^{\circ}$ & $1.01^{\circ}$ & $1.01 \mathrm{~m}^{\circ}$ \\
Theoretical torque & $27 \mathrm{Nmm} / \mathrm{MPa}$ & $3.4 \mathrm{Nm} / \mathrm{MPa}$ & $1.8 \mathrm{Nm} / \mathrm{MPa}$ \\
Effective torque & $3.8 \mathrm{Nmm} / \mathrm{MPa}$ & $2.0 \mathrm{Nm} / \mathrm{MPa}$ & $1.1 \mathrm{Nm} / \mathrm{MPa}$ \\
Efficiency & $14 \%$ & $60 \%$ & $59 \%$ \\
Max. power & $40 \mathrm{~mW}$ & $800 \mathrm{~mW}$ & $4.2 \mathrm{~mW}$
\end{tabular}

\section{Step size validation results}

The number of steps per revolution of the R-10 and R-40 were counted and found to be equal to 28 and 356, respectively, which are in accordance with the theoretical values.

The R-54 was left running for one hour at a stepping frequency of $99 \mathrm{~Hz}$. At five instances a photo was taken of the output shaft from which the angle was extracted using graphics software. These angles are plotted in Fig. 5.8. The angular velocity was measured to be $0.0987^{\circ} / \mathrm{s}$, at a stepping frequency of $99 \mathrm{~Hz}$ this is equivalent to a step size of $0.997 \mathrm{~m}^{\circ}$ and consistent with the theoretical value of $1.011 \mathrm{~m}^{\circ}$, up to the measurement error.

\subsubsection{Comparison and Discussion}

Table 5.1 lists the specifications and measured characteristics of the R-10, R40 and R-54 motors. Fig. 5.9 graphically shows the build sizes and step sizes 


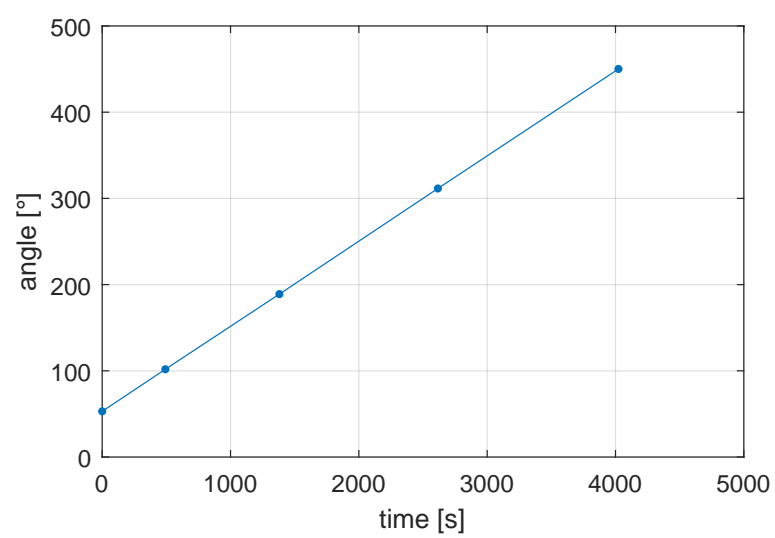

Figure 5.8: Observed angle of the R-54 as function of elapsed time.

of the R-10, R-40 and R-54 in red so that these dimensions can be directly compared to state-of-art motors.

The R-10 is smaller than any other motor by a considerable margin and this shows that it is possible to construct a miniature MR safe pneumatic stepper motor measuring only $10 \mathrm{~mm}$ in every direction (excluding axle). The measured torque of $1.2 \mathrm{Nmm}$ is relatively low. The mechanical efficiency is only $14 \%$ which can be attributed to several factors. The finite 3 -D printing resolution results in blunt teeth tips in the pistons resulting in a suboptimal wedge mechanism [52]. Another important factor is the high perimeter-tosurface ratio which causes relatively high friction in the moving parts, reducing the effective force of the cylinders. Still, a further reduction in size may be possible by optimizing the design and production methods, but reduction gears will be needed in practical applications.

The R-40 motor combines a practical step size and adequate torque in a housing which is still compact enough for use in MR safe surgical robotics. The build volume is comparable to that of several state-of-art designs, but the step size of $1.01^{\circ}$ is smaller than any of these. The limiting factor here is the minimum gear teeth tip spacing needed for reliable meshing with the piston teeth taking the limitations of rapid prototyping into account.

The R-54 shows an extreme example of step size reduction by effectively exploiting the capabilities of the planetary gear. While the demonstrated step size of $1.01 \mathrm{~m}^{\circ}$ is too small to have any practical value in MR safe robotics, the step size equation shows that by carefully choosing the right gear sizes virtually any step size can be obtained. One application requiring relatively small step sizes (order of $0.1^{\circ}$ ) are steerable catheters for endovascular applications. The build volume of the R-54 is larger than that of the R-40, but there is sufficient 


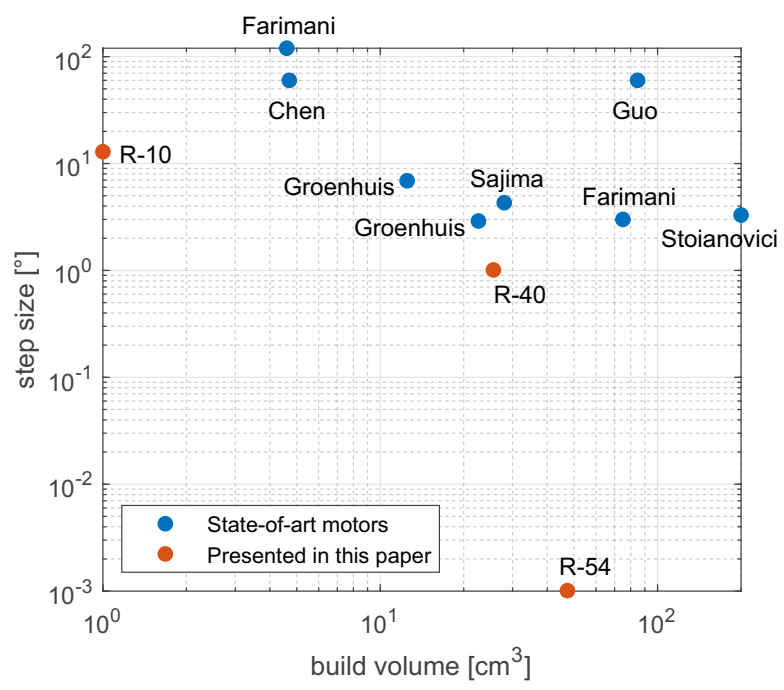

Figure 5.9: Graphical comparison of build volumes and step sizes of state-of-art motors (blue) and the three motors presented in this paper (red).

room for miniaturization. The planetary gear modulus could be reduced from 0.5 down to 0.33 and also the number of teeth on the external ring and carrier gears could be reduced, resulting in a stepper motor smaller than the R-40 but with broad customizability of step sizes.

The motors were tested with relatively short pneumatic tubes. In MR safe applications the valve manifold is typically placed outside the Faraday cage of the MRI scanner, requiring long $(5 \mathrm{~m})$ tubes between motors and manifold. This reduces the maximum stepping frequency to approx. $8 \mathrm{~Hz}$ without compromising on torque [47]; higher stepping frequencies cause a reduction in output torque due to the pneumatic transmission line characteristics.

The wedge mechanism involves sliding contact surfaces which are subject to friction losses and wear. This is mitigated by the use of low-friciton materials (acetal and glossy-printed VeroClear), the application of a suitable lubricant (petroleum jelly) and by involving multiple teeth per contact to better distribute the load. It has been shown that the amount of wear in certain designs is negligible [47] and the friction and wear can be further reduced by optimizing the material choice.

The R-40 and R-54 are inherently MR safe. The presented R-10 first needs to have its carbon fibre axle replaced by e.g. a fibreglass rod of the same diameter before it can also be classified MR safe. 


\subsection{Conclusion}

The three motors presented in this paper show that significant reduction in build volume and/or step size in MR safe pneumatic stepper motors is possible compared to state-of-art stepper motors. The R-10 only measures $10 \mathrm{~mm}$ in every direction, the R-40 offers a good compromise between build dimensions and step size and the R-54 demonstrates a very small step size of $1.0 \mathrm{~m}^{\circ}$ due to the integration of a high-reduction planetary gear.

The described developments offer several possibilities in future MR safe robotics. Small individual motors with different characteristics can be coupled to form dual-speed motors on every axis, hereby circumventing the low bandwidth associated with long pneumatic tubes. Miniature motors such as the R-10 could be integrated in the tip of an endoscope and offer a pneumatic alternative to conventional wire actuation techniques. Lastly, the planetary gearbox design in the R-54 enables customization of the step size tailored to the specific application. 



\section{Part III}

\section{MR safe robotic systems for breast biopsy}





\section{Controlling the Stormram 2: An MRI-compatible Robotic System for Breast BIOPSY}

M. E. Abdelaziz, V. Groenhuis, J. Veltman, F. Siepel, and S. Stramigioli. Controlling the Stormram 2: An MRI-compatible robotic system for breast biopsy. In Proceedings - IEEE International Conference on Robotics and Automation, pages 1746-1753, 2017. doi: 10.1109/ICRA.2017.7989206 


\subsection{Abstract}

Breast cancer is the most frequently life-threatening diagnosed type of cancer among women. Early and accurate diagnosis by acquiring a tissue sample using biopsy techniques is essential. However, small lesions only visible by MRI are often missed in standard methods, indicating the need for a roboticassisted biopsy system that is MRI-compatible. Existing proof-of-concepts are difficult to employ due to large sizes and/or actuation complexities. Therefore, a compact pneumatically-actuated 5 DOF MRI-compatible robot was further developed and controlled by a computerized valve manifold. Accuracy and efficiency measurements have been performed using two different PVC breast phantoms with embedded fish oil capsules (mimicking lesions) inside a $0.25 \mathrm{~T}$ MRI scanner. Preliminary results show that the end-effector was able to hit the targeted capsules, and that the position accuracy is in the range of 4.7$7.3 \mathrm{~mm}$. The developed robotic system has potential to perform MRI-guided breast biopsies accurately and improve the clinical workflow.

\subsection{Introduction}

Breast cancer is the most occurring invasive cancer among women, accounting for about $25 \%$ of all cases worldwide. Early diagnosis is essential for successful treatment. Because of the high sensitivity, MRI scanners can detect lesions (suspicious abnormalities) not found by mammography (x-ray), ultrasound or palpation. It may be necessary to perform a biopsy to extract tissue from such a lesion for accurate histological examination, requiring a needle to be inserted at the exact location, which is difficult to perform using MRI guidance. In standard procedures, the lesion is missed in approximately $10 \%$ of the cases [65], requiring repositioning of the needle leading to additional tissue damage, or possibly a false negative biopsy. Therefore, there is a request for robotic systems that can perform the biopsy more accurately and efficiently.

\subsubsection{State of the Art}

The MRI scanner requires that components are MRI-compatible and do not distort the magnetic field. As electromagnetic motors are ruled out, actuation of the robot is a major challenge. Alternatives have been found in pneumatics, hydraulics, Bowden tubes and piezoelectric actuation. Different MRIcompatible biopsy robots have been developed using (combinations of) these actuation systems. Hungr et al. developed a 5 DOF needle insertion robot driven by piezo motors coupled to the robot with Bowden tubes, combined with a pneumatic needle insertion system [70]. Su et al. developed different 


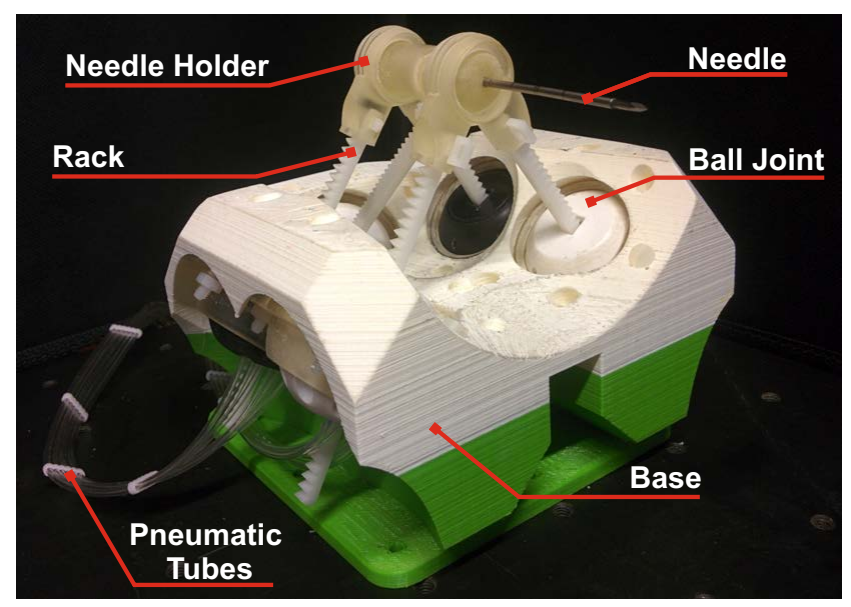

Figure 6.1: The Stormram 2 robot.

piezo/pneumatic-driven robots for prostate biopsy, of which the control electronics are placed in a shielded box inside the MRI room [122]. Yang et al. developed a piezo/pneumatic robotic system for breast biopsy [136]. Li et al. developed a transapical aortic valve replacement robot using the commercial Innomotion arm combined with a custom 3-DOF delivery module driven by pneumatic cylinders with optical feedback sensors [82]. The aforementioned robotic systems have in common that the actuation system is complex and expensive. Specialized electronics are required for driving piezoelectric actuators in a MRI environment, which also have to be located at sufficiently large distance from the scanning volume to avoid distortion of the MRI scans.

Therefore, research has been performed to develop pneumatically-driven robotic systems. Stoianovici et al. developed the six-DOF pneumaticallydriven MrBot [32] for prostate intervention, but construction and control of this system is complex. In contrast, the co-authors of this paper, Groenhuis and Stramigioli, developed a low cost 7 DOF robotic system for breast biopsy driven by pneumatic linear stepper motors [46], later called the Stormram 1. It was entirely rapid prototyped by laser-cutting and 3D printing and control is relatively straightforward, but it is too bulky to be practical and not computercontrolled yet. The Stormram 2 design described in this paper intends to solve both issues, by miniaturization and the development of a computerized controller.

The main aim of this study is to localize the lesion in the breast phantom using MRI, and guide the robotic assisted biopsy needle of Stormram 2 towards it. 
Ch 6: Controlling the Stormram 2: An MRI-compatible Robotic System for 108 Breast Biopsy

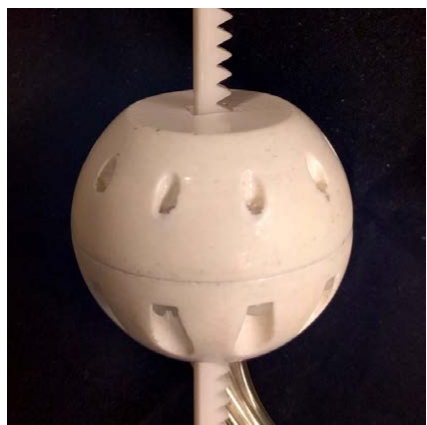

(a)

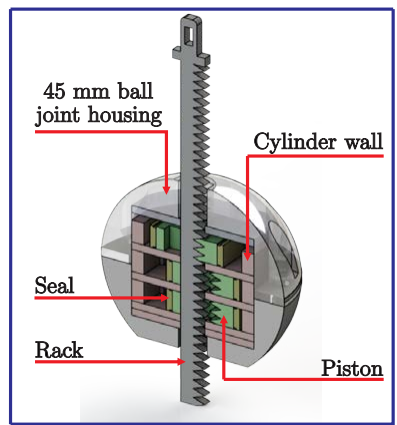

(b)

Figure 6.2: (a) Assembled stepper motor, (b) Cutaway labeled view.

\subsection{Design and Implementation}

To design for maximal MRI-compatibility, a fully plastic robotic system driven by non-electric actuators was designed without using any metallic components, except for the off-the-shelf titanium needle. The pneumatic linear stepper motor principle was chosen as the actuation method because previous research has shown that this is a suitable and straightforward method for driving MRIcompatible robots. The entire robot was rapid prototyped by $3 \mathrm{D}$ printing and laser-cutting.

\subsubsection{Overview}

The Stormram 2 robot consists of a frame, a needle holder and interconnecting links. The frame size is $140 \mathrm{~mm} \times 140 \mathrm{~mm} \times 95 \mathrm{~mm}$ and was printed as one piece with polyactic acid (PLA) material using the Ultimaker 2 machine (Ultimaker B.V., Geldermalsen, The Netherlands). The needle holder consists of six parts, all printed in FullCure720 photopolymer material using the Objet Eden250 machine (Stratasys Ltd., Eden Prairie, MN, USA). The needle holder is connected to the frame by means of a five-link parallel platform, which kinematic structure is inspired by MrBot [32]. Each link connects a ball joint in the frame to a joint in the needle holder. The distance between these two joints is controlled by a linear stepper motor. The off-the-shelf MRI-compatible 14-gauge titanium needle is the only metallic component of the robot, which consists of a shaft with outer diameter $2.1 \mathrm{~mm}$, and a removable inner stylet. 


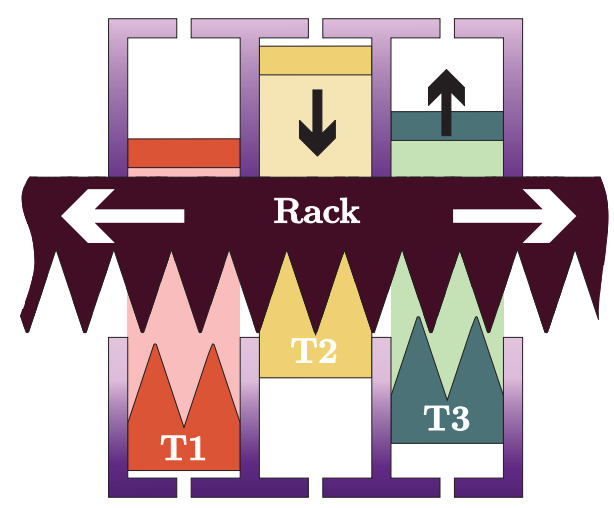

Figure 6.3: Operating principle of the three pistons acting on the rack.

\subsubsection{Stepper motors}

Pneumatic linear stepper motors actuate the Stormram 2 robot. The design is a miniaturization of the motors used in Stormram 1 as described previously [46], such that it can be embedded inside a $45 \mathrm{~mm}$ ball joint shown in Figure 6.2(a). It consists of two 3D printed hemispheres fixed together with nylon screws, enclosing a cavity with outer dimensions $29 \mathrm{~mm} \times 25 \mathrm{~mm} \times 26 \mathrm{~mm}$ for the motor mechanism.

\section{Motor construction}

The motor mechanism is entirely laser-cut from acetal and silicone rubber sheets. The internal structure can be seen in the cutaway view (Figure 6.2(b)). Several acetal parts (red) are stacked together to form three cavities in which toothed pistons (green) can slide. The pistons were manufactured by lasercutting from different sides, to obtain the designed shape of the teeth. Silicone seals (yellow) are loosely attached to the piston's heads and prevent leakage of air. The bore's cross-sectional area is $12 \mathrm{~mm} \times 5 \mathrm{~mm}=60 \mathrm{~mm}^{2}$. The piston's teeth engage on a rack (white) with pitch size $3 \mathrm{~mm}$. Six pneumatic tubes with length $7 \mathrm{~m}$ and inner diameter $1.4 \mathrm{~mm}$, guide pressurized air from the valve manifold to the chambers. The nominal operating pressure is $0.4 \mathrm{MPa}$.

\section{Principle of operation}

Figure 6.3 shows the principle of operation. The three pistons move up and down individually, according to the pressurization waveform of the six chambers. Initially, the center piston (yellow) is pushed against the rack. When the right piston (green) moves up and the center one retracts, the rack is pushed one step $(1 \mathrm{~mm})$ to the right due to the wedge mechanism in the piston-teeth 
interaction. More steps can be performed in either direction by applying appropriate waveforms to the six chambers. This way, the relative position of the rack can be controlled in feed-forward fashion in steps of $1 \mathrm{~mm}$ using the Pulse Width Modulation (PWM) technique, which in turn moves the robot's end-effector from one waypoint to another. When the initial position is known and no steps are missed, absolute position control is possible.

\section{Force calculation}

The force of the piston acting on the rack can be calculated as follows. When supplied with a pressure $P=0.4 \mathrm{MPa}$, the force is:

$$
F_{p}=P \cdot A=0.4 \cdot 10^{6} \cdot 60 \cdot 10^{-6}=24 \mathrm{~N}
$$

Due to the wedge mechanism, a piston displacement of $1.5 \mathrm{~mm}$ causes a rack displacement of $1.0 \mathrm{~mm}$. Assuming ideal transfer of work, the resulting theoretical force $F_{r}$ exerted by the rack due to the force of one piston is $F_{r}=$ $\frac{1.5}{1} F_{p}=36 \mathrm{~N}$.

Previous measurements [49] have shown that the actual force is $15 \mathrm{~N}$ at a pressure of $0.35 \mathrm{MPa}$. At a pressure of $0.4 \mathrm{MPa}$, the force was found to be $18 \mathrm{~N}$. The efficiency is thus $\frac{18}{36}=50 \%$, because of losses due to friction in the sliding parts, and pressure drops due to small leakages.

\subsubsection{Needle holder}

The needle holder's central shaft consists of two parts that are connected by a bayonet mount and accomodates a 14-gauge $(2.1 \mathrm{~mm})$ needle. At either end of the shaft, $\varnothing 18 \mathrm{~mm}$ three-way ball joints are installed. These joints consist

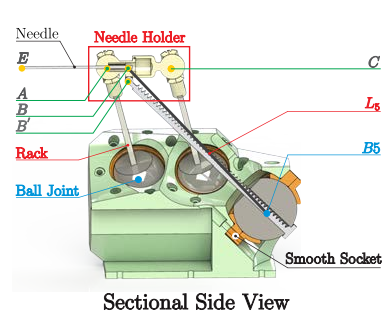

(a)

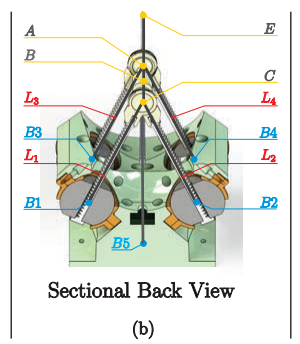

(b)

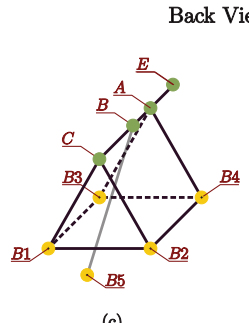

(c)

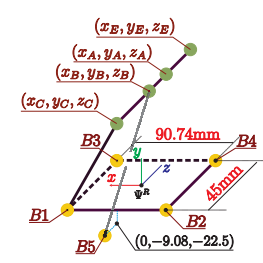

(d)

Figure 6.4: (a)-(b) Labeled SolidWorks assembly of Stormram 2 showing all the critical points of the needle holder, end-effector $\left(A, B, B^{\prime}, C\right.$ and $\left.E\right)$, the centre of the ball joints $(B 1, B 2, B 3, B 4$ and $B 5)$ and the connections between the points in different views. (c) Labeled sketch of Stormram 2's back view showing all the critical points (d) Labeled sketch of Stormram 2's back view showing the Robot Coordinate System $\left(\Psi^{R}\right)$, the coordinates of all points and other relevant dimensions. 
of three parts, shown in Figure 6.5. The ball part is enclosed by two identical socket parts that are interlocked by a revolute joint inspired by the bayonet mount. Because each socket part features both a rim and a groove, these can revolute over more than $180^{\circ}$ in the interlocked state. Each socket part in turn is rigidly attached to the rack of the stepper motor. The joint connecting rack 5 to the needle holder is a revolute joint, using a $3 \mathrm{~mm}$ screw as the pin (rack 5 connects points $B^{\prime \prime}$ and $B 5$

as shown in figure 6.4).

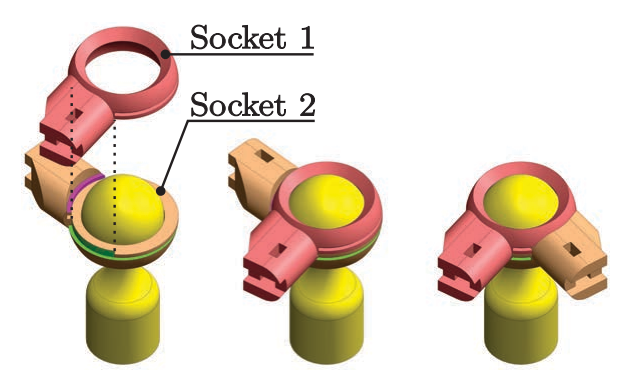

Figure 6.5: Three-way ball joint mechanism, consisting of one ball part and two interlocked socket halves.

\subsection{Kinematics}

\subsubsection{Inverse kinematics}

To derive the inverse position kinematics of Stormram 2, it is represented as a skeleton with nine critical points, namely:

- Four points of the needle holder : $E$ (End-Effector), $A, B$ and $C$.

- Five points representing the centres of the five $45 \mathrm{~mm}$ ball joints located in the base : $B 1, B 2, B 3, B 4, B 5$.

The variables $L_{1} . . L_{5}$ represent the lengths of the five racks. These define the distances from the centres of the ball joints $B 1 . . B 5$ to the corresponding needle holder points $A, B$ and $C$.

Point $B$ is located on the line connecting points $E, A$ and $C$. The actual joint connecting the rack 5 is situated at a lower point $B^{\prime}$. Therefore, $L_{5}$ should be the length between $B^{\prime}$ and $B 5$, however, to simplify the geometry of the robot, $L_{5}$ is approximated to the distance between points $B$ and $B 5$. This difference is compensated for in the software implementation of the inverse position kinematics. 
Figures 6.4 (a-d) illustrate the aforementioned points and variables in more detail. In figure 6.4(d), the Robot Coordinate System $\Psi^{R}$ uniquely determines the coordinates of the critical points. Furthermore, the five lengths $L_{1}, L_{2}, L_{3}, L_{4}$ and $L_{5}$ provide a desired end-effector $(E)$ position and orientation. Stormram 2 consists of five pneumatic linear stepper actuators and can therefore control the end-effector in five degrees of freedom, i.e. translation in $x, y, z$, rotation around $y$ (denoted by $\gamma$ ) followed by rotation around $x$ (denoted by $\alpha$ ) - as illustrated in figure 6.6.

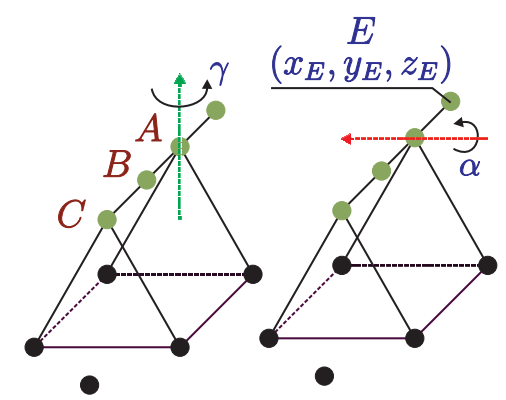

Figure 6.6: End-effector E Position and orientation.

The first step in determining the lengths $L_{1}, L_{2}, \ldots L_{5}$ from the given desired end-effector position $x_{E}, y_{E}, z_{E}$ and orientation $\gamma, \alpha$, is to obtain the relations between the needle holder's critical points $(A, B$ and $C$ ) and the end-effector point $E$. The relation between point $A$ and $E$ is given by:

$$
\begin{aligned}
& x_{A}=x_{E}-L_{A E} \sin (\gamma) \\
& y_{A}=y_{E}+L_{A E} \cos (\gamma) \sin (\alpha) \\
& z_{A}=z_{E}-L_{A E} \cos (\gamma) \cos (\alpha)
\end{aligned}
$$

where $\left(x_{A}, y_{A}, z_{A}\right)$ is the position of point $A$ and $L_{A E}$ is the length between points $A$ and $E$ with a value $62.43 \mathrm{~mm}$. Similarly, the relation between point $A$ and $C$ is given by:

$$
\begin{aligned}
& x_{C}=x_{A}-L_{A C} \sin (\gamma) \\
& y_{C}=y_{A}+L_{A C} \cos (\gamma) \sin (\alpha) \\
& z_{C}=z_{A}-L_{A C} \cos (\gamma) \cos (\alpha)
\end{aligned}
$$

where $\left(x_{C}, y_{C}, z_{C}\right)$ is the position of point $C$ and $L_{A C}$ is the length between points $A$ and $C$ with a value $45.83 \mathrm{~mm}$. Next, the position of point B is derived. 
From figure 6.4 and 6.6 , point B lies between points $A$ and $C$. In general the position of a point (in this case $B$ ) on a line segment $(A C)$ is given by:

$$
\begin{aligned}
x_{B} & =\lambda x_{C}+(1-\lambda) x_{A} \\
y_{B} & =\lambda y_{C}+(1-\lambda) y_{A} \\
z_{B} & =\lambda z_{C}+(1-\lambda) z_{A}
\end{aligned}
$$

where $\lambda$ is the ratio of the known length between points $A$ and $B$ (i.e. $L_{A B}$ ) with a value $15.07 \mathrm{~mm}$ to the known length $L_{A C}$.

$$
\lambda=\frac{L_{A B}}{L_{A C}}=\frac{15.07}{45.833}=0.3288
$$

With the position of points $A, B$ and $C$ defined and evaluated, the lengths between the aforementioned points and the known ball joint centers $(B 1, B 2, B 3, B 4, B 5)$ are defined using the distance between two points mathematical relation:

$$
\left(x_{m}-x_{n}\right)^{2}+\left(y_{m}-y_{n}\right)^{2}+\left(z_{m}-z_{n}\right)^{2}=\left(L_{k}\right)^{2}
$$

where $m=A, B, C, n=B 1 \ldots B 5$ and $k=1 \ldots 5$. The final step is to solve for the lengths $L_{1}, \ldots L_{5}$, given a desired $x_{E}, y_{E}, z_{E}, \gamma, \alpha$.

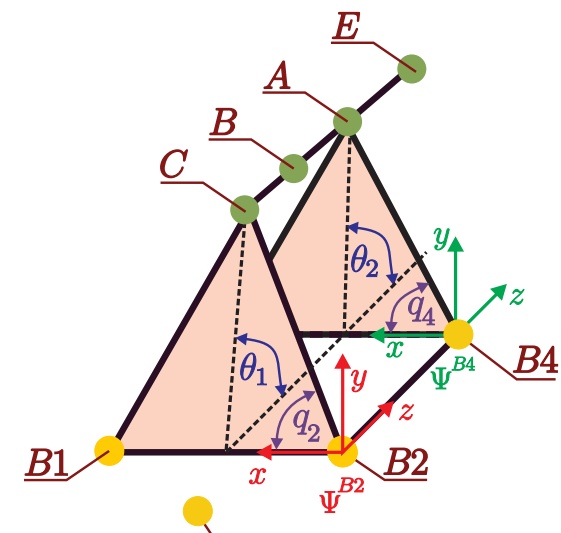

$\underline{B 5}$

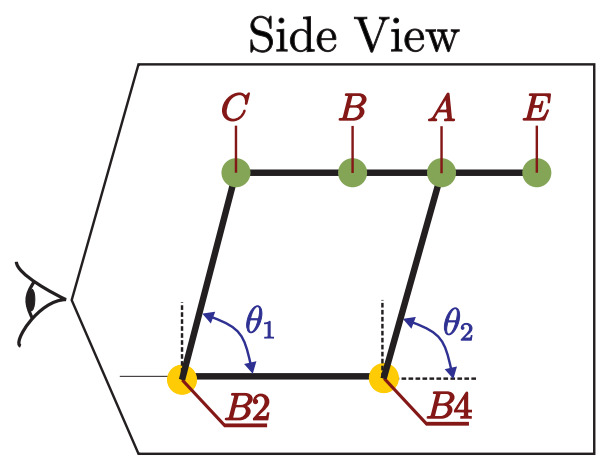

Figure 6.7: Labeled sketch of Stormram 2's back view showing all the critical points, two newly added parameters $q_{1}$ and $q_{2}$ and the two intermediate coordinate systems $\Psi^{B 2}$ and $\Psi^{B 4}$. 
Ch 6: Controlling the Stormram 2: An MRI-compatible Robotic System for 114

Breast Biopsy

\subsubsection{Forward kinematics}

In this section, the forward position kinematics of Stormram $\mathbf{2}$ is derived. The basic idea here is to determine the end-effector position based on given lengths $L_{1}, L_{2}, L_{3}, L_{4}$ and $L_{5}$. In order to obtain the forward kinematic relations, a direct approach would be to utilise the equations obtained in the previous section (Inverse Position Kinematics) and reformulate them to obtain 5 equations in terms of the end-effector Cartesian position and orientation $\left(x_{E}, y_{E}, z_{E}, \gamma, \alpha\right)$. This could be done by substituting equations (6.1) to (6.9) into equation (6.11) resulting in five highly non-linear simultaneous equations.

Solving the five highly non-linear equations is computationally expensive and therefore a more efficient approach is required. This is achieved by introducing two new parameters $\theta_{1}$ and $\theta_{2}$ into Stormram 2's kinematic relations and solving 2 equations instead of the 5 equations. Figure 6.7 illustrates the location of both parameters. In addition, two intermediate parameters $q_{1}$ and $q_{2}$ and two intermediate coordinate systems $\Psi^{B 2}$ and $\Psi^{B 4}$ are defined in order to obtain the final equations.

To begin with, using the cosine rule $q_{2}$ and $q_{4}$ are obtained as follows:

$$
\begin{aligned}
& q_{2}=\arccos \left(\frac{\left(L_{2}\right)^{2}+90.748^{2}-\left(L_{1}\right)^{2}}{2 \cdot L_{2} \cdot 90.748}\right) \\
& q_{4}=\arccos \left(\frac{\left(L_{4}\right)^{2}+90.748^{2}-\left(L_{3}\right)^{2}}{2 \cdot L_{4} \cdot 90.748}\right)
\end{aligned}
$$

Next, the position of critical point $A$ is obtained with respect to the coordinate system $\Psi^{B 4}$ and the position of critical point $C$ is obtained with respect to the coordinate system $\Psi^{B 2}$ :

$$
\begin{aligned}
& x_{A}^{B 4}=L_{4} \cos \left(q_{4}\right) \\
& y_{A}^{B 4}=L_{4} \sin \left(q_{4}\right) \sin \left(\theta_{2}\right) \\
& z_{A}^{B 4}=L_{4} \sin \left(q_{4}\right) \cos \left(\theta_{2}\right)
\end{aligned}
$$

$$
\begin{aligned}
& x_{C}^{B 2}=L_{2} \cos \left(q_{2}\right) \\
& y_{C}^{B 2}=L_{2} \sin \left(q_{2}\right) \sin \left(\theta_{1}\right) \\
& z_{C}^{B 2}=L_{2} \sin \left(q_{2}\right) \cos \left(\theta_{1}\right)
\end{aligned}
$$

To obtain the position vectors with respect to $\Psi^{R}$, Homogeneous matrices are utilised. The general form of the $4 \times 4$ homogeneous matrix is given by:

$$
H_{i}^{j}=\left[\begin{array}{cc}
R_{i}^{j} & o_{i}^{j} \\
\mathbf{0}^{T} & 1
\end{array}\right] \quad o_{i}^{j}=\left[\begin{array}{ccc}
o_{x_{i}}^{j} & o_{y_{i}}^{j} & o_{z_{i}}^{j}
\end{array}\right]^{T}
$$

where $R_{i}^{j}$ represents the $3 \times 3$ rotation matrix from coordinate system $i$ to coordinate system $j, o_{i}^{j}$ represents the $3 \times 1$ offset between the origins of the 
coordinate systems $i$ and $j$. The positions of points $A$ and $C$ are transformed as follows:

$$
\begin{aligned}
& p_{A}^{R}=H_{B 4}^{R} \cdot p_{A}^{B 4} \\
& p_{C}^{R}=H_{B 2}^{R} \cdot p_{C}^{B 2}
\end{aligned}
$$

where $p_{A}^{B 4}, p_{C}^{B 2}$ are the position vectors of points $A$ and $C$ with respect to the coordinate systems $\Psi^{B 4}$ and $\Psi^{B 2}$ respectively, $p_{A}^{R}, p_{C}^{R}$ are the position vectors with respect to the coordinate system $\Psi^{R}$ and $H_{B 4}^{R}, H_{B 2}^{R}$ are the homogeneous matrix transformations from coordinate systems $\Psi^{B 4}$ to $\Psi^{R}$ and $\Psi^{B 2}$ to $\Psi^{R}$ respectively.

$$
H_{B 4}^{R}=\left[\begin{array}{cc}
\mathbf{I} & o_{B 4}^{R} \\
\mathbf{0}^{T} & 1
\end{array}\right], \quad H_{B 2}^{R}=\left[\begin{array}{cc}
\mathbf{I} & o_{B 2}^{R} \\
\mathbf{0}^{T} & 1
\end{array}\right]
$$

In both cases, the rotation matrices $R_{B 2}^{R}$ and $R_{B 4}^{R}$ are identity matrices. This is because, the $x, y, z$ axes of all three coordinate systems are oriented in the same direction. The offset $o_{B 2}^{R}$ is the origin's position of coordinate system $\Psi^{B 2}$ with respect to the coordinate system $\Psi^{R}$ (the same applies for $o_{B 4}^{R}$ ). Furthermore, to satisfy the dimensions of the homogeneous matrix, the position vectors of critical points are written in the following manner:

$$
p_{v}^{l}=\left[\begin{array}{llll}
x_{v}^{l} & y_{v}^{l} & z_{v}^{l} & 1
\end{array}\right]
$$

where $p_{v}^{l}$ is arbitrary point $v$ defined with respect to coordinate system $l$. With the positions of points $A$ and $C$ defined with respect to the same coordinate system $\Psi^{R}$, the position of point $B$ is obtained in a similar manner to Equations (6.7) to $(6.9)$

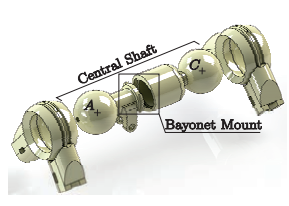

(a)

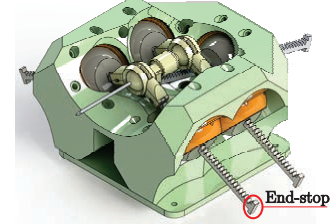

(b)

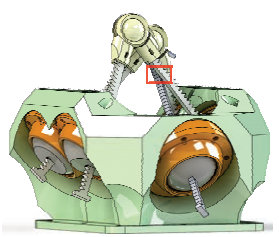

(c)

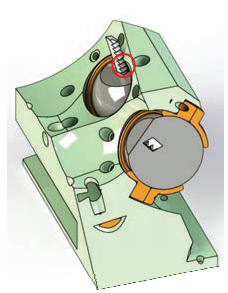

(d)

Figure 6.8: (a) Disassembled needle holder, (b) Stormram 2's racks' end-stops , Collisions between (c) racks (d) rack and the base. 
Ch 6: Controlling the Stormram 2: An MRI-compatible Robotic System for 116 Breast Biopsy

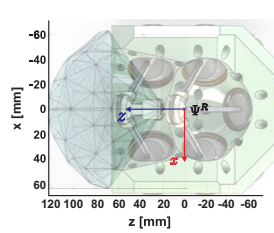

(a)

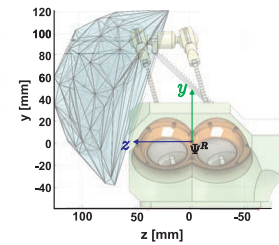

(b)

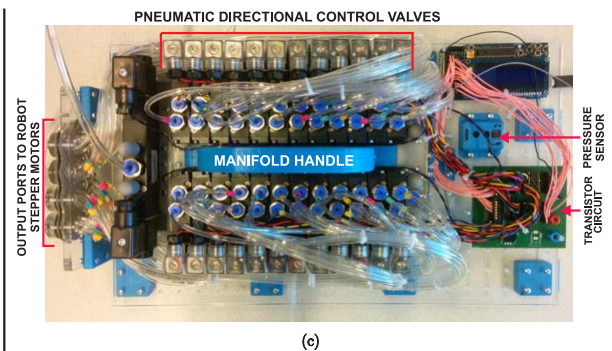

(c)

Figure 6.9: (a) Top view (b) Side view of Stormram 2's reachable workspace, (c) Computerized valve manifold.

$$
\begin{aligned}
x_{B}^{R} & =\lambda x_{C}^{R}+(1-\lambda) x_{A}^{R} \\
y_{B}^{R} & =\lambda y_{C}^{R}+(1-\lambda) y_{A}^{R} \\
z_{B}^{R} & =\lambda z_{C}^{R}+(1-\lambda) z_{A}^{R}
\end{aligned} \quad p_{B}^{R}=\left[\begin{array}{llll}
x_{B} & y_{B} & z_{B} & 1
\end{array}\right]^{T}
$$

In order to evaluate the forward kinematics, the following two equations are formulated: (1) The distance between points $p_{A}^{R}$ and $p_{C}^{R}$ and (2) The distance between points $p_{B}^{R}$ and $p_{B 5}^{R}$. For (1):

$$
\begin{aligned}
\left|p_{A}^{R}-p_{C}^{R}\right| & =L_{A C} \\
\left(x_{A}^{R}-x_{C}^{R}\right)^{2}+\left(y_{A}^{R}-y_{C}^{R}\right)^{2}+\left(z_{A}^{R}-z_{C}^{R}\right)^{2} & =\left(L_{A C}\right)^{2}
\end{aligned}
$$

For (2):

$$
\begin{aligned}
\left|p_{B}^{R}-p_{B 5}^{R}\right| & =L_{5} \\
\left(x_{B}^{R}-x_{B 5}^{R}\right)^{2}+\left(y_{B}^{R}-y_{B 5}^{R}\right)^{2}+\left(z_{B}^{R}-z_{B 5}^{R}\right)^{2} & =\left(L_{5}\right)^{2}
\end{aligned}
$$

Using the fsolve.m MATLAB function, the two simultaneous non-linear equations are solved for $\theta_{1}$ and $\theta_{2}$ given the five lengths $\left(L_{1}, L_{2}, L_{3}, L_{4}\right.$ and $\left.L_{5}\right)$ and initial estimates of $\theta_{1,0}=\theta_{2,0}=\pi / 2$. Once $\theta_{1}$ and $\theta_{2}$ are evaluated, the positions of critical points $A, B$ and $C$ are calculated. Next, since the endeffector point $E$ lies along the same line connecting points $A, B$ and $C$, and the length $L_{A E}$ is known, the position of $E$ can be evaluated in a similar manner to point $B$.

$$
\lambda_{2}=\frac{L_{A E}}{L_{A E}+L_{A C}} \quad x_{E}^{R}=\frac{x_{A}^{R}-\left(\lambda_{2} \cdot x_{C}^{R}\right)}{1-\lambda_{2}}
$$




$$
y_{E}^{R}=\frac{y_{A}^{R}-\left(\lambda_{2} \cdot y_{C}^{R}\right)}{1-\lambda_{2}} \quad z_{E}^{R}=\frac{z_{A}^{R}-\left(\lambda_{2} \cdot z_{C}^{R}\right)}{1-\lambda_{2}}
$$

\subsubsection{Robot Kinematic Constraints and Workspace}

For a parallel mechanism, like Stormram 2, the kinematic constraint analysis is rather complicated because of the dependency of the interconnected links and the collisions between the different components of the robot.
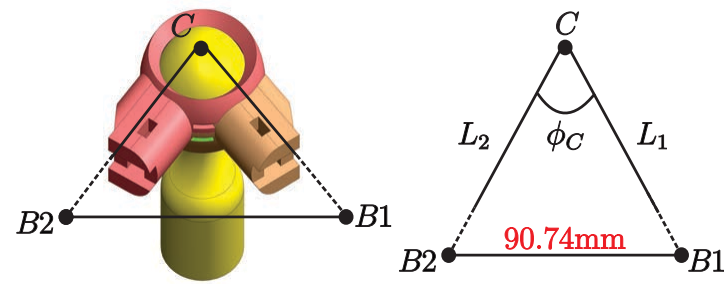

Figure 6.10: Labeled Solidworks model of Stormram 2 showing the critical points $C, B 1$ and $B 2$ and labeled schematic representation of the connection between the points. ( $90.74 \mathrm{~mm}$ is the measured length between points $B 1$ and $B 2$ in the x-direction)

The collisions appear between the following components: (1) Needle holder parts (sockets and central shaft - see figure 6.8(a)), (2) Racks' (interconnected links) end-stops and the ball joints (see figure 6.8(b) for the end-stops), (3) Racks (see figure 6.8 (c)), (4) Racks and the base of the robot (see figure 6.8(d)) and (5) Racks and the needle holder.

Figure 6.10 illustrates the kinematic constraint due to the restriction in the relative configuration between the sockets of the needle holder.

The angle defined in the figure above $: \phi_{C}$ is computed using the cosine rule, as shown below:

$$
\phi_{C}=\arccos \left(\frac{\left(L_{1}\right)^{2}+\left(L_{2}\right)^{2}-90.74^{2}}{2 \cdot L_{1} \cdot L_{2}}\right)
$$

Based on the design of Stormram 2, the minimum value of $\phi_{C}$ is $50^{\circ}$, i.e. $\phi_{C \min }=50^{\circ}$. A similar analysis is carried out on the remaining kinematic constraints and mathematical expressions (similar to 6.20) are obtained. Taking into account the kinematic constraint analysis and the forward position kinematics algorithm, the reachable space of the needle tip (point $E$ ) is investigated. The reachable workspace, defined as the set of reachable positions of point $E$, is a $3 \mathrm{D}$ pointcloud which is visualized in figure 6.9 (a) and (b). 

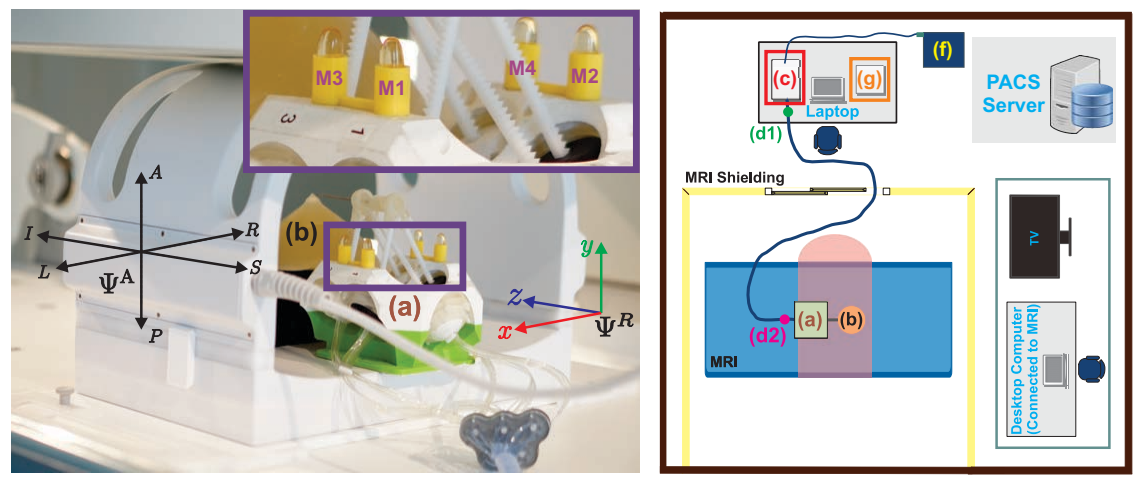

Figure 6.11: (a) MRI-compatible robotic breast biopsy system (Stormram 2) with 4 markers (M1 ... M4), (b) Breast phantom with markers, (c) Solenoid valves pneumatic distributor which is also referred to as the computerized valve manifold (see figure $6.9(\mathrm{c})),(\mathrm{d} 1$ and d2) Pneumatic tubes connecting the manifold to Stormram 2, (e) Portable Air Compressor, (f) MRI scanner - Esaote G-scan Brio 0.25T, (g) $24 \mathrm{~V} / 2.5 \mathrm{~A}$ power supply. $\Psi^{A}$ is the anatomical coordinate system (also called patient coordinate system).

\subsection{Measurements}

\subsubsection{Experimental setup}

Figure 6.11 illustrates an overview of the experimental setup. To conduct experiments, a breast phantom made of PVC with plasticizer, filled with fish oil capsules, was immobilized in a frame and placed in the MRI scanner (see figure 6.11). When scanning using the 3D Balanced Gradient Echo sequence in the 0.25T Esaote G-scan Brio MRI scanner, good contrast is visible between the phantom and fish oil capsules (see figure 6.12).

As illustrated in figure 6.11, the MRI-compatible robot (Stormram 2), equipped with four markers at known positions, is positioned next to the breast phantom. In order to drive the pneumatic stepper motors with appropiate waveforms, a computerized valve manifold (see figure 6.9 (c)) is developed to operate Stormram 2 and guide the biopsy needle. The manifold allows the user to operate the robot at a distance of up to 7 metres, which is required because the controller cannot be placed inside the MRI room itself. Consequently, transmission delays in the pneumatic lines restrict the motor's stepping frequency to approximately $5 \mathrm{~Hz}$, depending on the valves types and tube dimensions. The manifold is computer-controlled and operated by a graphical user interface designed and running in MATLAB. 


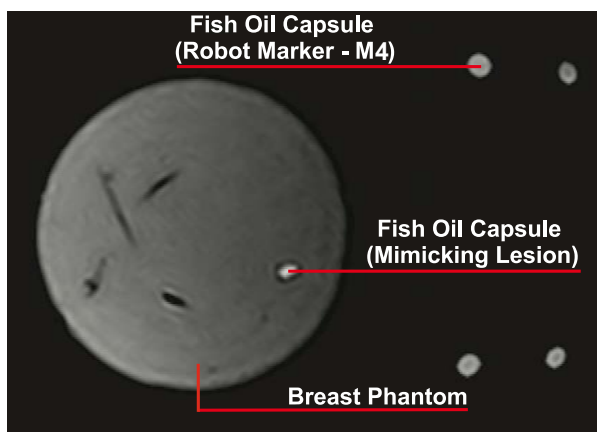

Figure 6.12: Labeled MRI image of Stormram 2 and the breast phantom.

\subsubsection{Method}

The experiments are conducted on two breast phantoms with different stiffnesses and lesions of different sizes, to emulate the physical differences between patients. In the stiff breast phantom (made of $100 \%$ (600 g) plastileurre), two $15 \mathrm{~mm}$-sized fish oil capsules are targeted. In the soft breast phantom (made of $85 \%(510 \mathrm{~g}$ ) of plastileurre and $15 \%$ (90 g) of assouplissant plastileurre), one fish oil capsule sized $7 \mathrm{~mm}$ is targeted.

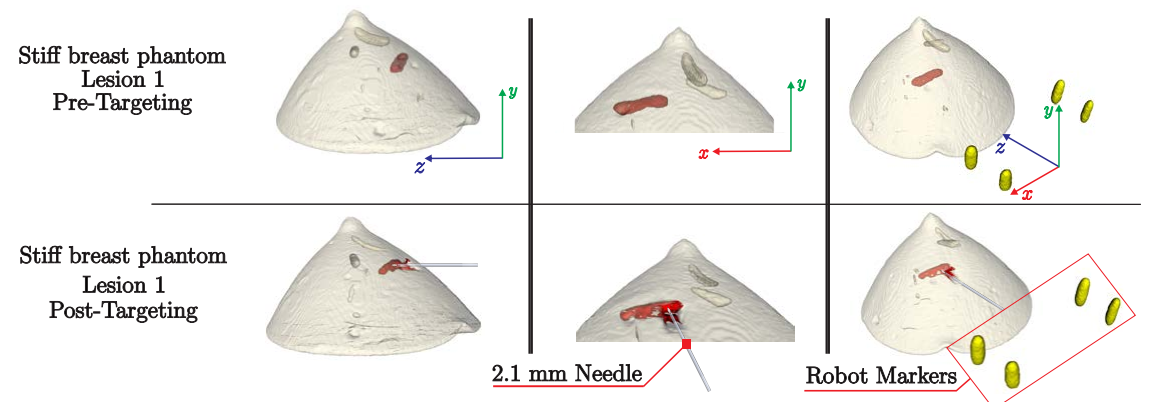

Figure 6.13: Rendering using 3D SLICER of the stiff breast, targeting the lesion with a size $15 \mathrm{~mm}$. 
Ch 6: Controlling the Stormram 2: An MRI-compatible Robotic System for 120 Breast Biopsy

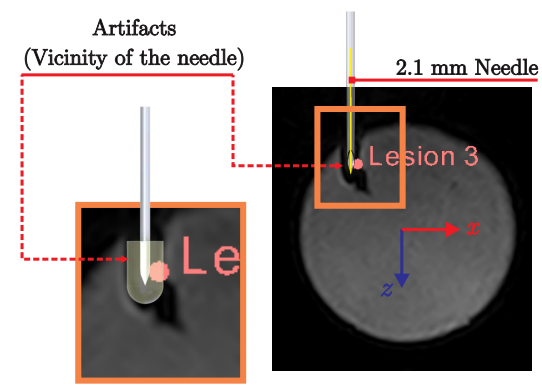

(a)

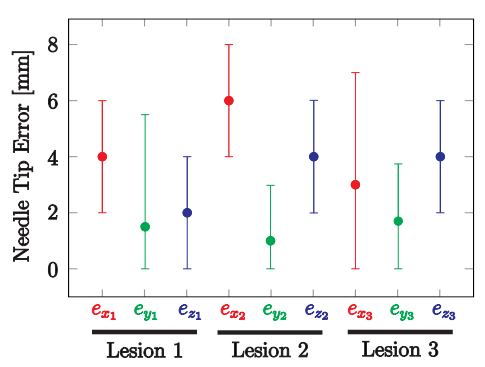

(b)

Figure 6.14: (a) The artifacts (yellow) in the MRI scan caused by the titanium (metallic) needle, (b) Error bar plot.

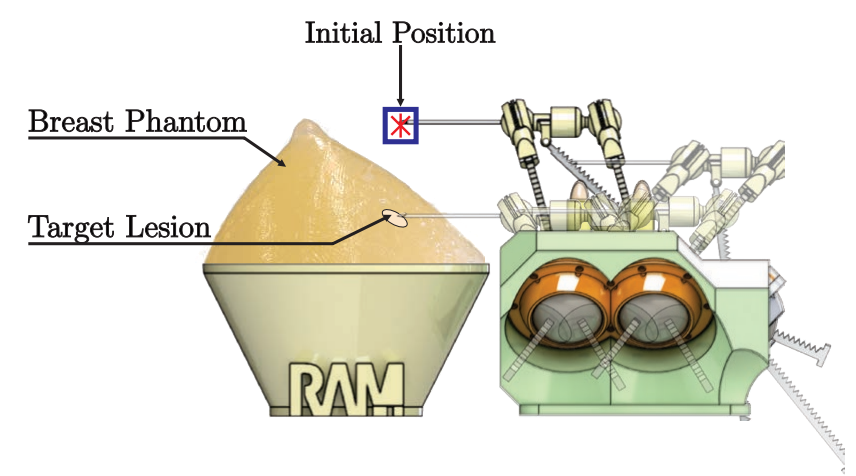

Figure 6.15: Robot and needle tip movement.

The following steps are taken: (1) localize lesion using MRI (pre-targeting scan), (2) compute desired end-effector (needle) position $\left(x_{E}^{R}, y_{E}^{R}, z_{E}^{R}, \gamma, \alpha\right),(3)$ apply inverse kinematics algorithm and obtain desired lengths $L_{1} \ldots L_{5}$, (4) actuate the pneumatic linear stepper motors to achieve computed lengths (see figure 6.15), (5) take another MRI scan (post-targeting scan), (6) check if endeffector (needle tip) targeted the lesion, and perform accuracy analysis.

The efficiency of the system is also investigated, by recording the time taken for the computer-controlled MRI guided localization procedure.

\subsubsection{Results}

Figure (6.13) illustrates the pre- and post-targeting segmented scans of the first lesion in the stiff breast phantom. Different views are shown to illustrate what happens once the needle enters the breast phantom and targets the lesion 
(marker). It can be observed that the needle has pierced the capsule.

Figure 6.14 (left) shows one MRI slice. The target lesion is projected on it. A black gap can be seen in the vicinity of the target lesion coordinate. This gap measures approximately $8 \mathrm{~mm}$ in width, while the needle is only $2.1 \mathrm{~mm}$. The needle is projected on the MRI slice in the center of the gap, as we can assume that the artifact is symmetrical around the needle. The distance between the needle tip and the target lesion in all 3 axes can now be derived, which is a measure of the tip accuracy. There is also an uncertainty in the measured needle tip location. For each of the three targeted lesions, the $x, y$ and $z$ components of the distance between the measured needle tip position and target lesion, along with its uncertainties, are plotted.

The needle successfully targeted all three lesions with a resultant error $e_{r}=$ $\sqrt{\left(e_{x}\right)^{2}+\left(e_{y}\right)^{2}+\left(e_{z}\right)^{2}}$ ranging from 4.7 and $7.3 \mathrm{~mm}$ with an uncertainty of approximately $2 \mathrm{~mm}$.

The time taken for the procedure was measured to be approximately 31 minutes.

\subsection{Discussion}

Needle tip positioning errors of approximately $6 \mathrm{~mm}$ were measured. These errors are a result of:

- Possible breast tissue deformations.

- Approximation of length $L_{5}$ (see figure 6.4) and misalignment of points $B$ and $B^{\prime}$.

- Clearances in the ball joints adding up to $2 \mathrm{~mm}$ backlash in the needle tip.

- $\pm 1 \mathrm{~mm}$ calibration error in each axis with regards to the homing procedure, leading to approximately $2 \mathrm{~mm}$ needle tip positioning error.

- An average segmentation error of $1 \mathrm{~mm}$.

- Discretization of the racks because of the $1 \mathrm{~mm}$ step size, causing needle tip position errors ranging from $0.3 \mathrm{~mm}$ to $8.3 \mathrm{~mm}$, with an average error of $0.8 \mathrm{~mm}$. There are two possible ways to reduce this error, namely: (1) reducing the step size, and (2) sweeping over different combinations $L_{1} \ldots L_{5}$ in the neighbourhood of the computed lengths combination, in order to optimize the lengths values, minimize error and achieve sub-mm accuracy.

The time needed to take a good quality 3D Balanced Gradient Echo scan with the $0.25 \mathrm{~T}$ scanner is approximately 5:30 minutes, plus 2:30 minutes postprocessing. Taking two complete scans therefore takes 16 minutes. This can 
Ch 6: Controlling the Stormram 2: An MRI-compatible Robotic System for 122 Breast Biopsy

be reduced significantly by using a higher field strength MRI scanner (1.5T or more) or by optimizing the scanning sequence. The robot could also be operated at higher speed by using a different software architecture. This way, the 32 minutes could be reduced to 12 minutes.

\subsection{Conclusion}

An innovative, pneumatically actuated 5 DOF MRI-compatible breast biopsy system with computerized controller has been developed. It was able to target lesions in a breast phantom with an accuracy of approximately $6 \mathrm{~mm}$ within 31 minutes.

The developed system has the potential to improve accuracy and efficiency of current MRI-guided breast biopsy procedures, reducing patient discomfort and the number of false negative biopsies.

\subsubsection{Future improvements}

A good fixation system is necessary to avoid movements of the breast. Also, a biopsy gun firing mechanism needs to be built in the system in order to perform the actual biopsy. Improvements in accuracy are possible by redesigning the homing procedure and smaller stepper motor step sizes, while the procedure time can be reduced by using quicker MRI scans and a more efficient software architecture. Furthermore, the kinematic design could be improved to increase the workspace to the full volume of the breast. 


\section{STORMRAM 3: An MRI-COMPATIBLE ROBOTIC SYSTEM FOR BREAST BIOPSY}

V. Groenhuis, J. Veltman, F. Siepel, and S. Stramigioli. Stormram 3: A Magnetic Resonance Imaging-Compatible Robotic System for Breast Biopsy. IEEE Robotics and Automation Magazine, 17(May):34-41, 2017. doi: 10.1109/MRA. 2017.2680541 


\subsection{Abstract}

Stormram 3 is an MRI-compatible robotic system that can perform MR guided breast biopsies of suspicious lesions. The base of the robot measures $160 \times 180 \times 90 \mathrm{~mm}$ and it is actuated by five custom pneumatic linear stepper motors, driven by a valve manifold outside the Faraday cage of the MRI scanner. All parts can be rapidly prototyped with 3-D printing or laser-cutting, making the design suitable for other applications such as actuation in hazardous environments. Based on the choice of materials, the robot (with the exception of the needle) is inherently MR-safe. Measurements show that the maximum force of the T-49 actuator is $70 \mathrm{~N}$, at a pressure of $0.3 \mathrm{MPa}$. The Stormram 3 has an optimized repeatability which is lower than $0.5 \mathrm{~mm}$, and can achieve a positional accuracy in the order of $2 \mathrm{~mm}$.

\subsection{Introduction}

\subsubsection{Clinical problem}

Breast cancer is the most prevalent invasive cancer among women, accounting for about $25 \%$ of all cases worldwide. Early detection of malignant lesions is essential for successful treatment. Palpation can be used for self-examination, but its sensitivity is limited. To provide wider early detection of breast cancer, screening programs utilizing imaging techniques have been set up in many countries. For example, mammography can be used to produce detailed 2D $\mathrm{x}$-ray images and is routinely used in periodic screening programs, with good sensitivity. Ultrasound (US) is also used to detect and analyze lesions in realtime.

MRI is an imaging technique with the highest sensitivity of lesion identification. Women with increased risk of breast cancer due to family history, and women with unspecific complaints may be offered MRI screening. MRI is also used to determine the optimal strategy for breast cancer surgery, as it can be used to determine whether a known cancer has metastatized to other parts of the breast.

When a suspicious lesion is found, a biopsy is needed for histopathological confirmation. The lesion is targeted with a biopsy needle, usually under ultrasound guidance, after which samples are collected. If the lesion is only visible on MRI and not on ultrasound, then the biopsy has to be performed under MRI guidance.

The current procedure of manual MRI-guided biopsy is a time consuming and costly procedure. The breast is first immobilized using fixation plates. After the initial scan and lesion localization, the patient is moved out of the scanner. The needle is then inserted blindly using a mechanical guide and a 


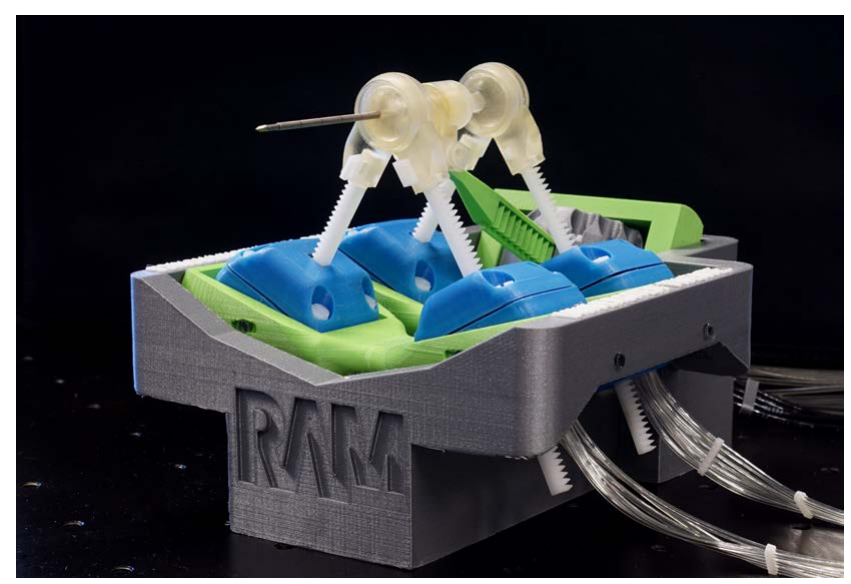

Figure 7.1: The MRI-compatible biopsy robot Stormram 3.

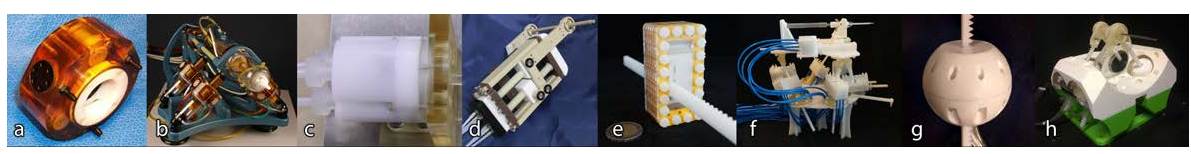

Figure 7.2: State-of-the-art pneumatic stepper motors and robots: a. PneuStep rotational motor and b. MrBot by Stoianovici et al. [119]; c. Rotational motor and d. Two-DOF manipulator by Sajima et al. [109, 110]; e. Linear motor and f. Stormram 1 robot by Groenhuis and Stramigioli [46]; g. Linear motor and h. Stormram 2 by Groenhuis et al. [49].

confirmation MRI scan is acquired. No imaging feedback is possible outside the scanner, which makes it difficult to compensate for unintentional tissue movements due to respiration and needle-tissue interactions. Therefore, needle placement inaccuracies must be compensated for by removing a large amount of tissue material causing additional discomfort. There is a need for improved systems for MRI-guided biopsy which are more precise and allow real-time imaging feedback, implying the need for an MRI-compatible robotic device.

\subsubsection{MRI-compatible actuators}

The MRI scanner contains a strong magnetic field and utilizes radiofrequency signals. Ferromagnetic metals and electromagnetic signals inside the Faraday cage of the MRI scanner can distort the images, especially when placed near the scanning volume. Therefore, for optimal imaging quality the use of ferromagnetic and conductive materials and electronic devices have to be avoided or minimized.

One of the key challenges in creating an MRI-compatible device is actuation. As electromagnetic motors are ruled out, many alternatives have been 
explored: hydraulic [133], piezo [93, 122], cable transmission [24], MRI-driven [40], air turbine [37], flexible fluidic actuators [30], direct-acting pneumatic actuators [135], unidirectional pneumatic stepper motors [27], and especially bidirectional pneumatic stepper motors $[26,46,49,60,109,110,113,118]$. Bidirectional pneumatic stepper motors have several important advantages: control is relatively straightforward by using a standard pneumatic valve manifold, a position feedback system is not necessary (provided that steps are never skipped), small leakages are acceptable because the medium is atmospheric air, and motors are scalable by changing cylinder cross-sectional areas.

\subsubsection{MRI-compatible biopsy robots}

A range of MRI-compatible biopsy robots have been developed. Su et al. developed multiple piezo/pneumatic-driven robots for prostate biopsy [124], in which the control electronics are placed in a shielded box inside the Faraday cage of the MRI scanner. Yang et al. [136] developed a piezo/pneumatic robotic system for breast biopsy. Stoianovici et al. developed the PneuStep (Figure 7.2a), a rotary stepper motor that is used for actuation of the MrBot (Figure 7.2b), designed for prostate biopsy interventions [118]. Sajima et al. also developed a rotary stepper motor (Figure 7.2c), for actuation of a two-DOF needle guiding manipulator (Figure 7.2d) [109, 110].

The authors of this paper developed a linear stepper motor (Figure 7.2e) for the Stormram 1 (Figure 7.2f), a 7 DOF needle manipulator based on the Stewart platform plus a needle insertion module [46]. The motor was minitiaturized in order to fit inside a $45 \mathrm{~mm}$ ball joint (Figure $7.2 \mathrm{~g}$ ), upon which the more compact, 5 DOF Stormram 2 robot was built (Figure 7.2h) [49].

The Stormram 2 demonstrated that it is possible to target lesions in a phantom breast with an accuracy of 6 millimetres [4]. Significant error sources arose due to clearances of the 3D printed ball joints, and in the coarse step size $(1 \mathrm{~mm})$ of the linear stepper motors. Furthermore, the workspace was limited and the motor forces insufficient to reliably operate the needle in dense tissue. The Stormram 3 robot was developed to address these shortcomings.

\subsection{Design and implementation}

Figure 7.1 shows the implementation of the Stormram 3, Figure 7.3a shows a 3D rendering and Figure 7.7 shows a kinematic diagram. Like its predecessor Stormram 2, it is a five-link parallel manipulator. It consists of one base (grey), three carriers (green), four blue stepper motors (blue with white racks), one T49 stepper motor (grey with green rack) and a needle holder consisting of seven components (yellow/orange). The design and implementation of the different parts, motors and joints are described in this section. 


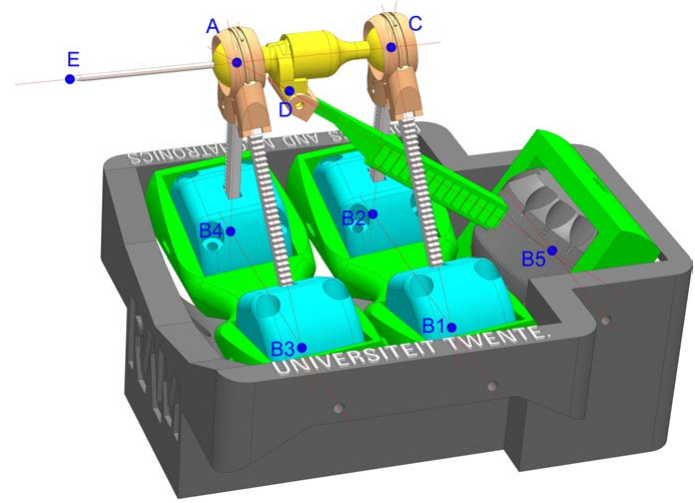

(a) Rendering with location of joint centroids $A, B 1 . . B 5$, $C$ and $D$, and needle tip $E$.

Figure 7.3: Stormram 3 kinematics.

\subsubsection{Base and carriers}

The base and carriers were printed with the Ultimaker 2 (Ultimaker BV, Geldermalsen, The Netherlands) in polyactic acid (PLA) material. Pin joints $J 9, J 6$ and $J 12$ connect the front, middle and rear carriers to the base. Pin joints $J 7, J 8, J 10, J 11$ and $J 13$ connect the five stepper motors to their carriers. All these joints are made of $4 \mathrm{~mm}$ acrylic rods, lubricated with petroleum jelly.

\subsubsection{Stepper motors}

For actuation of the five prismatic joints $J 1 . . J 5$, two different types of pneumatic linear stepper motors are used. Four copies of the blue motor, of which the centroids are located at positions $B_{1} . . B_{4}$, actuate joints $J 1-J 4$. The actuated joint $J 1$, for instance, controls the distance between points $C$ and $B_{1}$. The more powerful T-49 motor with centroid at position $B_{5}$ actuates joint $J 5$, driving the needle forwards and backwards.

\section{Blue stepper motor}

The blue stepper motor, shown in Figure 7.4a, is used to actuate four of the five degrees of freedom of the needle holder. The functional design is almost identical to that of the $45 \mathrm{~mm}$ spherical stepper motor (Figure $7.2 \mathrm{~g}$ ) used in the Stormram 2 robot [49]. The differences are that the step size is reduced from $1.0 \mathrm{~mm}$ to $0.67 \mathrm{~mm}$ for increased accuracy, and the motor's housing changed from a $45 \mathrm{~mm}$ sphere to a cylinder sized $\varnothing 48 \mathrm{~mm}$ x $34 \mathrm{~mm}$ with holes for the pin 


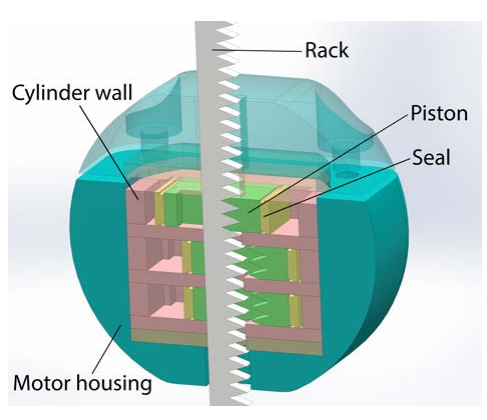

(a) Blue motor cutaway.

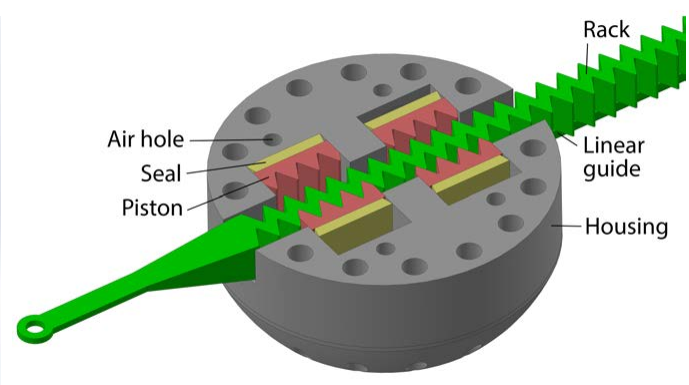

(b) T-49 motor cutaway view.

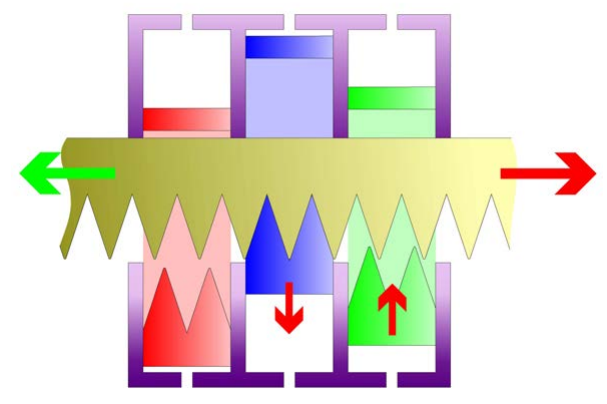

(c) Principle of operation of blue motor.

Figure 7.4: Details of blue and T-49 stepper motors.

joint. This eliminated the excessive clearance problem of the original $45 \mathrm{~mm}$ ball joints.

The stepper motor's internal mechanism (Figure 7.4a) consists of lasercut acetal parts, according to the design principles stated in Groenhuis and Stramigioli [46]. The cylinder case (pink) consists of seven plates that are stacked, forming three cavities in which toothed pistons (green) can slide, sealed by silicone seals (yellow). By pressurizing either chamber, the piston is pushed to the opposite side. The pistons themselves are laser-cut in a special way: after cutting out the top-view shape, the pistons are placed upright in a frame so that the teeth can be laser-cut from the side, resulting in a shape with the correct tooth geometry. The rack is also laser-cut from acetal; having a crosssection of $3.5 \times 4.0 \mathrm{~mm}$, these racks are somewhat flexible. This property has no influence on the rigidity of the needle holder, as forces are transferred in longitudinal direction only.

The bore's cross-sectional area $A$ is $12 \times 5 \mathrm{~mm}=60 \mathrm{~mm}^{2}$ and operating pressure $P=0.3 \mathrm{MPa}$, so the theoretical force $F$ exerted by the pistons (ignoring friction losses) is $F=P \cdot A=0.3 \cdot 10^{6} \cdot 60 \cdot 10^{-6}=18 \mathrm{~N}$. The three pistons 
interact with a rack of teeth with a pitch of $2 \mathrm{~mm}$ and a depth of $2.5 \mathrm{~mm}$. The interaction is by means of a wedge mechanism: a piston displacement of $2.5 \mathrm{~mm}$ causes a rack displacement of $1.0 \mathrm{~mm}$, so the wedge factor is $\alpha=\frac{2.5}{1.0}=2.5$. Assuming ideal transfer of work (no energy dissipation or storage), the theoretical force exerted by the rack is calculated to be $2.5 \cdot 18=45 \mathrm{~N}$ (ignoring friction losses). The actual force has to be measured with a proper setup, and the difference between theoretical and actual force can be attributed to friction and pressure losses.

The principle of operation of the complete motor is shown in Figure 7.4c. When the green piston is pushed up and the blue one retracted, the rack moves one step to the right. This occurs because the three piston's jaws are phased $120^{\circ}$ apart. By pressurizing the six chambers with appropiate waveforms, the position of the rack can be controlled in steps of $0.67 \mathrm{~mm}$. When all three pistons are retracted, the rack can slide freely, which might be useful when considering medical robot safety in a clinical setting.

\section{T-49 stepper motor}

A new motor, the T-49 stepper motor, has been developed to drive the needle forwards and backwards. This motor measures $\varnothing 56 \times 40 \mathrm{~mm}$ and a CAD design is given in Figure 7.4b. It consists of six different custom parts: three housing parts, two identical pistons, four silicone seals and the rack. The silicone seals were hand-cut using a 3D printed mechanical guide, all other parts were 3D printed with the Ultimaker 2 in PLA material.

The novel part is that the T-49 has only two cylinders, in which pistons act on a double-sided rack. Also, the motor is fully $3 \mathrm{D}$ printed, eliminating the need of a separate housing required for the blue motor. In this way, the available space can be used more efficiently and the increased bore's cross-sectional area results in higher output forces. This cross-sectional area is $14 \times 14 \mathrm{~mm}=$ $196 \mathrm{~mm}^{2}$. Both teeth pitch and teeth depth are set to $4 \mathrm{~mm}$. The step size is one quarter of the pitch, i.e. $1 \mathrm{~mm}$. A piston displacement of $4 \mathrm{~mm}$ causes a rack displacement of $2 \mathrm{~mm}$, so the wedge factor is $\alpha=\frac{4.0}{2.0}=2.0$. When pressurized with a pressure of $P=0.3 \mathrm{MPa}$, the theoretical force exerted by the rack (ignoring friction losses) is $F_{r}=2.0 \cdot 0.3 \cdot 10^{6} \cdot 196 \cdot 10^{-6}=118 \mathrm{~N}$, which is over 2.5 times the force of the blue motor. This makes inserting the needle in dense tissue and stiff lesions easier. Needle insertion forces in excess of $10 \mathrm{~N}$ may be needed in such cases.

The two pistons have a total of four jaws, phased $90^{\circ}$ apart. One drawback is that it is not possible to arrange the pistons in a configuration that allows free sliding of the rack, although it is possible to move the rack manually by applying a certain force, when the chambers are all de-pressurized. 

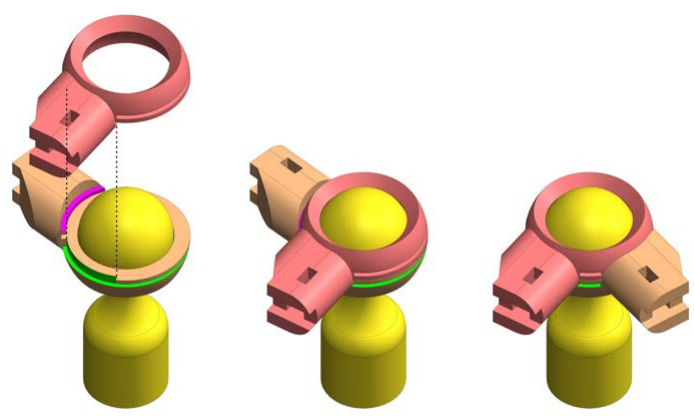

Figure 7.5: The combined ball/revolute joint, in three stages of assembly. The red and orange parts are identical and both feature a rim (magenta) and groove (green). Left: the rim is aligned with an opening in the groove. Middle: parts matched, but not secured yet. Right: parts effectively interlocked, resulting in a combined ball/revolute joint.

\subsubsection{Needle holder}

The needle holder consists of seven pieces that are printed with the Stratasys Objet Eden 250 (Stratasys Ltd, Eden Prairie, MN, USA) in FullCure720 material. The central shaft, connecting points $A, C$ and $D$ together, consists of two parts that are connected by a bayonet mount and accomodate a 12-gauge (2.1 mm) needle.

Joints $J 16$ and $J 17$ are combined ball/revolute joints, that consist of three parts, shown in Figure 7.5. The ball part (ball radius $9 \mathrm{~mm}$ ) is enclosed by a pair of identical socket halves that are interlocked by a revolute joint inspired by the bayonet mount. Each of the two socket parts are rigidly attached to the racks of the stepper motors in the same carrier.

Finally, joints $J 14$ and $J 15$ are pin joints, together forming a universal joint which connects the rack of joint J5 to the needle holder. As for the base and carriers, $\varnothing 4 \mathrm{~mm}$ acrylic rods connect the different parts together.

\subsubsection{Pressure distributor}

In order to drive the pneumatic stepper motors with appropiate waveforms, a pneumatic distributor was required. A manually-controlled distributor was developed for the Stormram 1 robot [46], and is also used to operate the Stormram 2 and 3 by visual servoing. As it is practically impossible to guide a biopsy needle in breast tissue by visual servoing alone, in the absence of realtime 


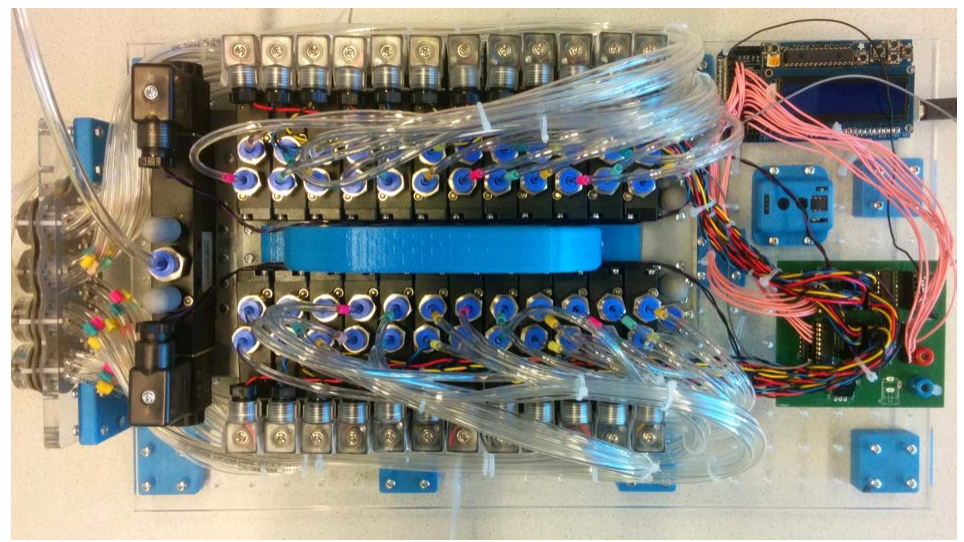

Figure 7.6: Computer-controlled pneumatic distributor.

MRI, a computerized valve manifold (Figure 7.6) was also built. This manifold can control the different robots programmatically towards given target coordinates in feed-forward fashion. Both manifolds allow operation of the robot at a distance of 7 metres, as required because the controller cannot be placed inside the Faraday cage of the MRI scanner itself due to electromagnetic interference. Consequently, airflow limitations and transmission delays in the pneumatic lines restrict the motor's stepping frequency to approximately $5 \mathrm{~Hz}$ for the manual manifold [46], and $10 \mathrm{~Hz}$ for the computerized one.

\subsection{Kinematics and workspace}

In order to steer the needle along the desired trajectory towards a specific target, knowledge of the robot kinematics is required. In this section, the forward and inverse kinematics are described. The desired end-effector configurations can be translated to actuator configurations, and vice versa.

\subsubsection{Coordinate system}

Figure 7.7 shows the kinematic diagram of Stormram 3 and defines two coordinate frames. Frame $\Psi_{0}$ is attached to the base of the robot, and $\Psi_{E}$ is attached to the needle holder. The coordinate transformation from $\Psi_{0}$ to $\Psi_{E}$ is represented as $H_{0}^{E}$. The Stormram 3 pose is fully defined by this transformation, as all joint centroids are rigidly attached to either $\Psi_{0}$ or $\Psi_{E}$.

The actuated joint configuration vector $\mathbf{v}=\left(\ell_{1}, \ell_{2}, \ell_{3}, \ell_{4}, \ell_{5}\right)$ defines the state of the five prismatic actuators. A length $\ell_{i}$ represents the distance between the joint centroids that are linked by the $i$-th prismatic joint. 


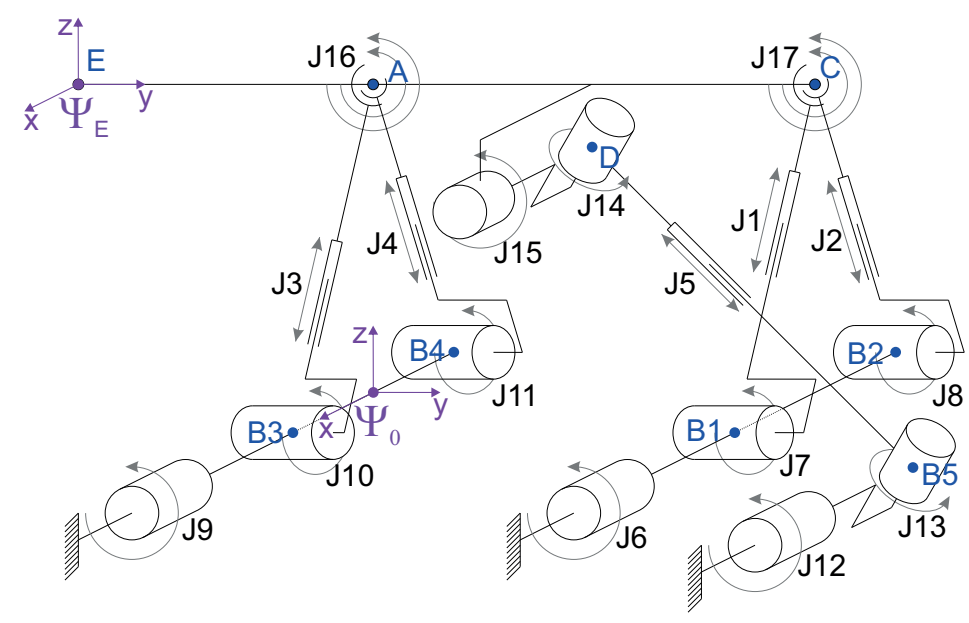

Figure 7.7: Kinematic diagram of Stormram 3, with coordinate systems $\Psi_{0}$ and $\Psi_{E}$.

The forward kinematics problem can now be defined as finding $H_{0}^{E}$ from a given $\mathbf{v}$. The inverse kinematics problem involves finding $\mathbf{v}$ for a given $H_{0}^{E}$ with one free parameter, because only five degrees of freedom can be controlled.

\subsubsection{Forward kinematics}

As the Stomram 3 is a parallel manipulator, derivation of $H_{0}^{E}$ from a given $\mathbf{v}$ is not trivial. A 3D coordinate transformation has six degrees of freedom. Therefore, six constraints are needed to define $H_{0}^{E}$.

The first step is to constrain the points $A$ and $C$ to one degree of freedom. We can observe that the triangle $\triangle A B_{3} B_{4}$ defines a plane, intersecting the $X Y$ plane in $\Psi_{0}$ along the $B 3-B 4$ line. As for a given $\mathbf{v}$, all sides of this triangle are determined, point $A$ must lie on a circle around the $B 3-B 4$ axis with known midpoint and radius (given by the lengths of the $\triangle A B_{3} B_{4}$ triangle). Likewise, point $C$ must lie on another circle around the $B 1-B 2$ axis, with origin and radius given by the lengths of the $\triangle C B_{1} B_{2}$ triangle.

The second step is to fix the distance between points $A$ and $C$ as defined by the needle holder geometry, constraining the number of DOFs of $\Psi_{E}$ to two because the orientation of $\Psi_{E}$ around its $Y$-axis is still free.

The third step is to apply the constraint put by the serial kinematic chain $J 12-J 13-J 5-J 14-J 15$. This chain has four free parameters (as $J 5$ is fixed), thus fixing $(6-4)=2$ degrees of freedom of $\Psi_{E}$, making the Stormram 3 a well-determined kinematic system. Unfortunately, there is no elegant way to express this last constraint mathematically without having to write out the full kinematic chain with all its joints. 
To get a practically workable system for forward kinematics, it is proposed to start with an approximation, combined with an estimation of the error. The approximation is based on the observations that $A D$ and $D B_{5}$ are approximately collinear and $D$ is relatively close to $A$. Thus, one can approximate $A B_{5}$ as follows:

$$
\left\|A B_{5}\right\| \approx\left\|B_{5} D\right\|+\|A D\|
$$

With an approximation of the error $\varepsilon$ :

$$
\varepsilon \approx\|A D\|\left(1-\cos \angle B_{5} A D\right)
$$

All coordinates can now be calculated with basic goniometry methods. It is possible to reduce the error by iteratively repositioning $A$ based on the actual distance $A B_{5}$, but if the exact solution is needed then a more computationally intensive approach is required.

\subsubsection{Inverse kinematics}

The inverse kinematics problem states finding the joint vector $\mathbf{v}=\left(\ell_{1}, \ell_{2}, \ell_{3}, \ell_{4}, \ell_{5}\right)$ for a given end-effector configuration $H_{0}^{E}$ with one free parameter, chosen to be the rotation around the $Y$-axis in the $\Psi_{E}$ coordinate frame because the needle is axially symmetric.

Derivation of the coordinates of points $A$ and $C$ is trivial, and the lengths $\ell_{1} . . \ell_{4}$ can be calculated directly. The fifth length, $\ell_{5}$, can be approximated or numerically solved analogous to the forward kinematics problem problem.

Stepper motors are discrete, so in general $\mathbf{v}$ is not in the joint configuration space $V$. The blue stepper motor has a $0.67 \mathrm{~mm}$ step size, and for the T-49 motor it is $1.0 \mathrm{~mm}$. Ignoring constant offsets, we have $\mathbf{v}^{\prime}=\left(\frac{2}{3} n_{1}, \frac{2}{3} n_{2}, \frac{2}{3} n_{3}, \frac{2}{3} n_{4}, n_{5}\right)$ with $n_{i} \in \mathbb{N}$. A simplistic solution is to use the nearest values for $\mathbf{v}_{i}$ in $V$, but this might result in a significant error in the end-effector position. A better approach takes into consideration that needle tip positional accuracy takes precedence over angle accuracy, so when a range of insertion angles is possible, the one which minimizes the end-effector position error can be chosen.

\subsubsection{Workspace calculation}

Figure 7.8 shows a visualization of the reachable workspace of the needle tip, taking the most important physical constraints into account. The voxel size is $2.0 \times 2.0 \times 2.0 \mathrm{~mm}$ and the workspace is the union of all voxels that the needle tip can reach. The volume was found to be $2.0 \mathrm{~L}$, but due to unmodeled physical constraints, the actual volume is slightly smaller. 


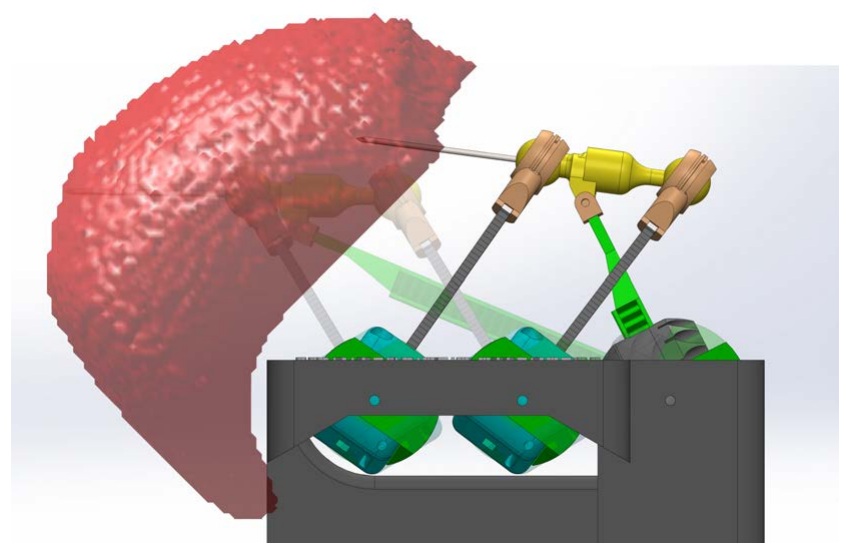

Figure 7.8: Visualization of the workspace (red), with the Stormram 3 shown in two extreme configurations.

\subsection{Measurements}

Several measurements were performed to assess the performance of the joints, the two motor types and the repeatability of the Stormram 3 robot.

Extensive quantitative accuracy measurements on breast phantoms inside a MRI scanner were performed with the Stormram 2 robot controlled by the computerized manifold. These experiments are described in Abdelaziz et al. [4]. Based on an analysis of these experiments and repeatability measurements of the Stormram 3, the positional accuracy of Stormram 3 is estimated.

\subsubsection{Friction and clearances in joints}

The pin joints in the base and carriers, joint $J 6-J 13$, were found to have no measurable backlash. There is no clearance around the pins, and the structure of the base, carriers and motors are sufficiently rigid to limit parasitic movements below $0.1 \mathrm{~mm}$. The static friction in the joints were measured to be up to $0.02 \mathrm{Nm}$.

The combined ball/revolute joints in the needle holder have a certain clearance. When the joints are dry, this results in parasitic movements in the order of $0.2 \mathrm{~mm}$. After lubrication with petroleum jelly the parasitic movements are reduced to below $0.1 \mathrm{~mm}$ during normal operation.

\subsubsection{Repeatability measurements}

The repeatability of the Stormram 3 was evaluated using the manual valve manifold and a sheet of paper rigidly attached to a fixed object. By sequentially 


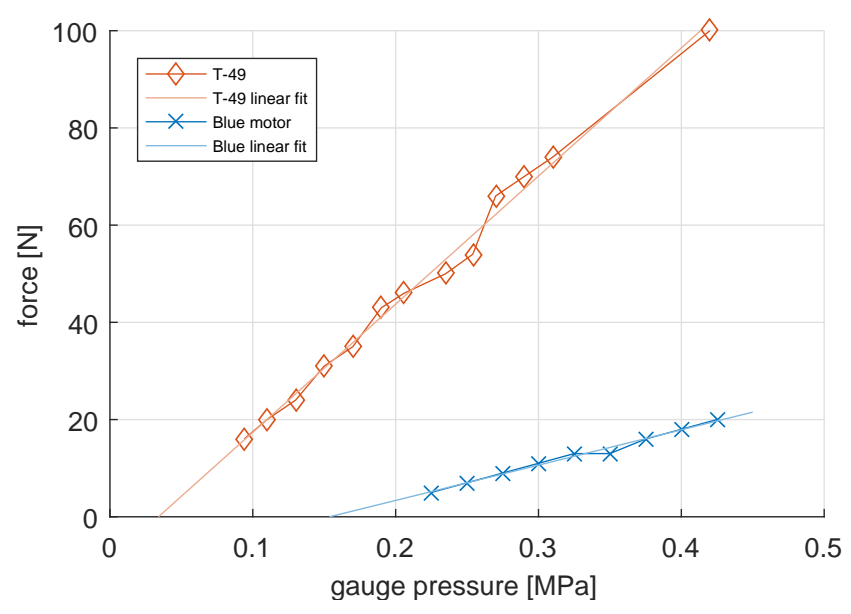

Figure 7.9: T-49 and blue motor force versus pressure.

adjusting rack lengths $\ell_{1} . . \ell_{5}$ by a known number of steps, the needle pierced holes with diameter $0.5-1.0 \mathrm{~mm}$ at five different locations on the sheet. By repeatedly moving the needle to the same joint configurations (taking care of hysteresis in the joints by keeping the approach consistent), it was observed that the needle always enters a hole that was already formed in this particular joint configuration. The result is that the Stormram 3 has a repeatability of better than $0.5 \mathrm{~mm}$.

\subsubsection{Stepper motor measurements}

The blue and T-49 stepper motors were characterized by measuring its maximum pulling force as a function of the gauge pressure. Figure 7.9 shows their pressure-force relationship. At a pressure of $0.3 \mathrm{MPa}$, which is the standard working pressure of Stormram 3, the blue motor exerts $16 \mathrm{~N}$ of force while the T-49 can exert $70 \mathrm{~N}$ of force. The calculated theoretical values were $45 \mathrm{~N}$ and $118 \mathrm{~N}$, respectively. The resulting mechanical efficiency is $36 \%$ for the blue motor, and $59 \%$ for the T-49 motor.

\subsubsection{MRI tests}

Qualitative MRI tests were performed with the Esaote G-scan Brio 0.25 T scanner. A phantom breast made of PVC with plasticizer, filled with fish oil capsules, was immobilized in a frame and placed in the MRI scanner. The Stormram 3 robot was positioned adjacent to the frame, and operated using visual servoing to insert the needle and examine any artifacts. See Figure 7.10 for the 


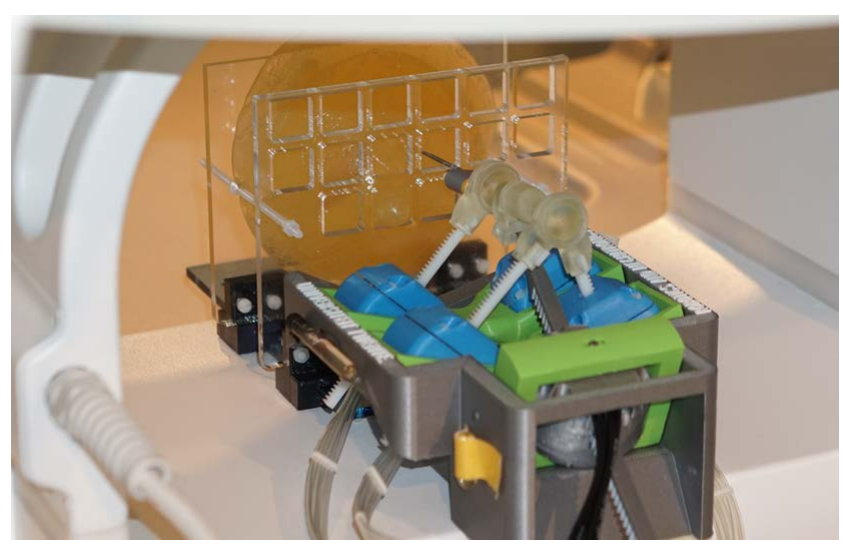

Figure 7.10: Stormram 3 in the $0.25 \mathrm{~T}$ MRI scanner.

test setup, Figure 7.11a for one MRI slice and Figure 7.11b for a 3D rendering showing the phantom and a few markers.

The Stormram 3 robot (without needle) was found to have no measurable influence on MRI scans. After equipping it with a MRI-compatible, 14-gauge $(2.1 \mathrm{~mm})$ titanium needle and inserting it in a phantom, a susceptibility artifact up to a distance of $4 \mathrm{~mm}$ around the needle was observed.

Extensive quantitative measurements were performed with the Stormram 2 robot, controlled by the computerized manifold. These measurements are described in Abdelaziz et al. [4] and the average targeting error was found to be $6 \mathrm{~mm}$.

\subsection{Discussion}

The Stormram 3 robot is a significant improvement over its predecessor. The T-49 motor can exert $70 \mathrm{~N}$ of force, making it suitable for targeting lesions in dense tissue. Due to the different kinematic design, the workspace has been increased and parasitic movements in the joints in the base have been effectively eliminated.

In ex-vivo MRI tests, the Stormram 2 has shown to have a targeting accuracy of $6 \mathrm{~mm}$ on average [4]. This error can be attributed to parasitic movements in joints, geometry approximations in the kinematics, discretization of the racks, hysteresis in linear stepper motors, offsets in calibration, needle deflection due to needle-tissue interaction and needle position measurement inaccuracies. Of these errors, the parasitic movements in joints have been effectively eliminated in the Stormram 3, and discretization errors have been reduced due to the smaller step size of $0.67 \mathrm{~mm}$. Inaccuracies in kinematic 


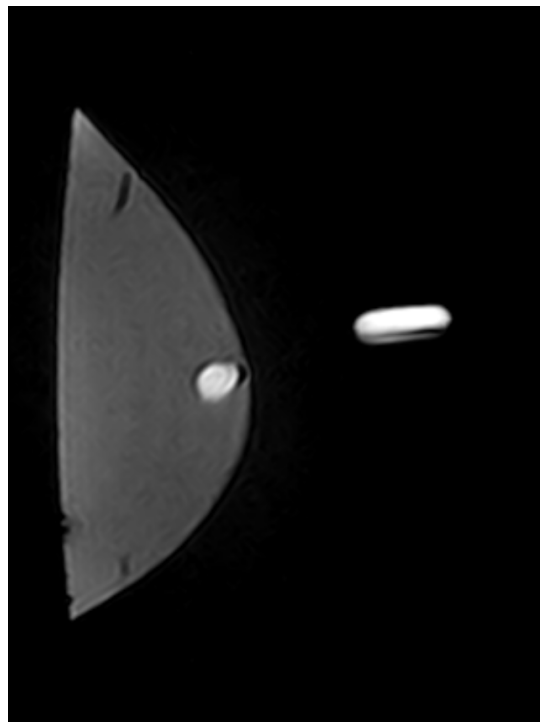

(a) One MRI slice, visualizing markers in the breast phantom and attached to the (invisible) needle.

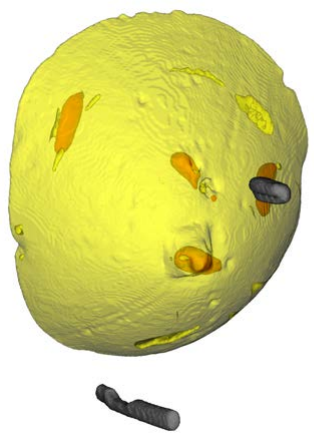

(b) Rendering of phantom (yellow) with embedded markers (orange), and markers attached to the Stormram 3 base (grey), rendered by $3 \mathrm{D}$ Slicer software.

Figure 7.11: MRI test setup and scanning results.

calculations, calibration and hysteresis can be solved by a more sophisticated software implementation that precisely models the kinematics and actuator characteristics of the robot. In this way, and taking into account the repeatability of better than $0.5 \mathrm{~mm}$, it is expected that the Stormram 3 can have a needle-tip positioning accuracy of as small as $2 \mathrm{~mm}$.

The Stormram 3 robot (without needle) is entirely made from materials (plastics, silicone, nylon, photopolymer) that are considered to be safe with regard to the $\mathrm{MR}$ environment, and therefore does not distort the resulting MR images.

The kinematic design of the Stormram 3, with the five-link parallel actuator, is similar to that of the MrBot (Figure 7.2b). One important difference is that the MrBot has one extra degree of freedom to translate the needle holder longitudinally. This is essential in obtaining smooth, linear needle trajectories in biological tissue, and to obtain a sufficiently large workspace. While it is possible to develop this for the Stormram 3 as well, it also makes the needle less accessible, complicating the biopsy procedure.

Several additional steps are required to advance the Stormram 3 further towards a complete market product that can potentially replace the current manually-operated MRI-guided breast biopsy procedure. A biopsy needle firing 
mechanism should be embedded, which can be triggered by the radiologist without the need to open the robot to swap needles. A comfortable patient bed with good fixation mechanism is essential. Software that combines preoperative MRI scans with a needle path trajectory planning system and postinsertion validation needs to be developed. This software should also be able to take care of breast deformations, which could be performed in several ways. By using an elastographic model of the breast, deformations could be simulated and be accounted for. An alternative approach would be to acquire one or more intermediate scans during insertion of the needle, allowing to adjust the path based on these near-realtime scans.

\subsection{Conclusion}

The Stormram 3 has shown that pneumatic linear stepper motors are an effective way of actuating MRI-compatible robots. Excluding the off-the-shelf needle, the robot is inherently MRI-compatible by the choice of materials. It can be driven by either a manually-operated pneumatic distributor using visual servoing, or programmatically using a computer-controlled valve manifold. The robot has shown to have sufficiently high stiffness and negligible parasitic movements. The needle tip repeatability is better than $0.5 \mathrm{~mm}$, and based on the evaluation of the accuracy of Stormram 2, it is expected that in MRI experiments with the Stormram 3 using more sophisticated software, the needle tip positioning error can be kept in the order of $2 \mathrm{~mm}$.

The technology developed in Stormram 3 is not limited to applications in MRI-compatible breast biopsy robots. Biopsies in prostate and other surgical interventions under MRI guidance may also benefit from a compact MRIcompatible robot driven by pneumatic linear stepper motors. As the powerful T-49 pneumatic stepper motor is relatively easy to produce and assemble, it may also find its way to applications outside the MRI scanner whenever pressurized air supply is available. 
CHAPTER 8

\section{Design and Characterization of Stormram 4: An MRI-Compatible Robotic System for BREAST BIOPSY}

V. Groenhuis, F. J. Siepel, J. Veltman, and S. Stramigioli. Design and characterization of Stormram 4: an MRI-compatible robotic system for breast biopsy. In IEEE/RSJ International Conference on Intelligent Robots and Systems (IROS) September 24-28, 2017, Vancouver, BC, Canada, volume 2017-Septe, pages 1746-1753, 2017. doi: 10.1109/IROS.2017.8202256 
Ch 8: Design and Characterization of Stormram 4: An MRI-Compatible

Robotic System for Breast Biopsy

\subsection{Abstract}

Targeting of small lesions with high precision is essential in an early phase of breast cancer for diagnosis and accurate follow up, and subsequently determines prognosis. Current techniques to diagnose breast cancer are suboptimal, and there is a need for a small, MRI-compatible robotic system able to target lesions with high precision and direct feedback of MRI. Therefore, the design and working mechanism of the new Stormram 4, an MRI-compatible needle manipulator with four degrees of freedom, will be presented to take biopsies of small lesions in the MRI scanner. Its dimensions (excluding racks and needle) are $72 \times 51 \times 40 \mathrm{~mm}$, and the system is driven by two linear and two curved pneumatic stepper motors. The T-26 linear motor measures $26 \times 21 \times 16 \mathrm{~mm}$, has a nominal step size of $0.25 \mathrm{~mm}$ and the measured maximum force is $63 \mathrm{~N}$ at $0.65 \mathrm{MPa}$. The workspace has a total volume of $2.2 \mathrm{~L}$.

Accuracy measurements have shown that the mean positioning error is $0.7 \mathrm{~mm}$, with a reproducibility of $0.1 \mathrm{~mm}$. Velocity measurements with $5 \mathrm{~m}$ long tubes show a maximum stepping frequency of $8 \mathrm{~Hz}$ (maximum force) to $30 \mathrm{~Hz}$ (unloaded). These results show that the robot might be able to target lesions with sub-millimeter accuracy within reasonable time for the MRI-guided breast biopsy procedure.

\subsection{Introduction}

\subsubsection{Clinical challenge}

Breast cancer is one of the most frequently diagnosed cancer types with an estimated 1.67 million new cancer cases in 2012, and the leading cause of cancer-related death among women worldwide [100]. In breast cancer screening, suspicious lesions need to be biopsied for pathological confirmation of the diagnosis. Some abnormalities are occult on mammography and ultrasound and can only be detected with MRI. In these cases a biopsy will be taken of the, often small, suspicious lesion under MRI-guidance. In current clinical practice, the needle must be inserted manually with the patient moved in and out of the scanner-bore multiple times for position adjustment and verification $[29,105]$.

This phase of the biopsy procedure is time-consuming and because of deformations due to needle-tissue interactions and patient movements, the needle may need to be re-positioned by using an alternate trajectory or multiple insertions leading to additional tissue damage and inaccurate placement [129].

Increased needle positioning accuracy and efficiency using a robotic system could improve the standard of care for women with a MRI detected lesion. If such a system is able to insert the needle inside the MRI scanner and is MRI- 


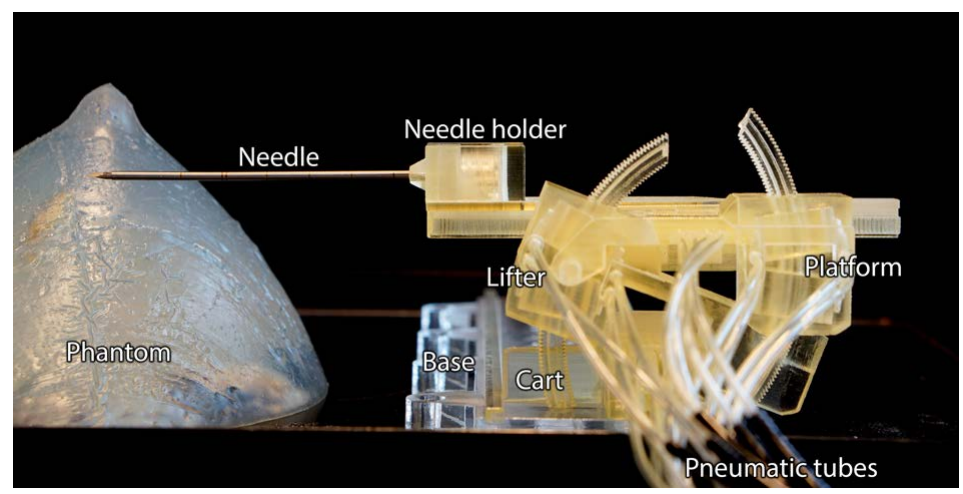

Figure 8.1: The Stormram 4 robot.

compatible itself, (near-)realtime imaging feedback is possible and enhances accuracy. Therefore, the aim of this project was to design and characterize an MRI-compatible robotic system for breast biopsy.

\subsubsection{State-of-art}

Previous studies showed the design of robotic systems in several applications inside the MRI scanner. Stoianovici et al. developed the MrBot [118], a six DOF robotic system for prostate biopsy and driven by pneumatic rotational stepper motors. These motors have good specifications, but are also relative and complex to build. Bomers et al. developed a five DOF robot for prostate biopsy guidance [15], driven by pneumatic screw-motion stepper motors which, while useful in this application, are difficult to use in smaller scale robotics. Franco et al. developed a four DOF robot for liver biopsy, driven by relatively large pneumatic cylinders with a time-delay control scheme [41], which is difficult to control precisely due to the complex pneumatic model. Hungr et al. developed a five DOF robotic system for liver biopsy [70]. This system is driven by a combination of ultrasonic motors, Bowden cables and pneumatic actuators, making it also relatively complicated to develop.

The authors of the current paper, Groenhuis et al., developed three robotic systems for breast biopsy. The Stormram 1 is a seven DOF needle manipulator driven by $72 \mathrm{~mm}$-sized pneumatic linear stepper motors [46] with a force of 24 N. For the Stormram 2, the motors were miniaturized to fit inside $45 \mathrm{~mm}-$ sized ball joints [49] and driven by a computerized valve manifold [4]. The Stormram 3 has five degrees of freedom, improved accuracy and workspace, and utilizes the T-49 stepper motor which can exert $100 \mathrm{~N}$ so that more dense tissue can be targeted.

The described robots are all parallel manipulators. While such a kinematic 
Ch 8: Design and Characterization of Stormram 4: An MRI-Compatible

Robotic System for Breast Biopsy

chain increases structural rigidity, it also limits the workspace and makes forward/inverse kinematics relatively complicated. The Stormram 3 also cannot move the needle along a straight path.

\subsubsection{Approach}

Our approach is to use a serial kinematic chain, driven by a combination of linear and novel curved pneumatic stepper motors. If rigidity can be preserved under the absence of metallic materials, a serial kinematic chain offers important advantages in terms of structural/kinematic complexity, controllability and workspace size. The robot can be made inherently MRI safe by the choice of materials and using pneumatic actuation.

\subsection{Design and Implementation}

\subsubsection{Robot design}

The presented Stormram 4 robot is a needle manipulator with four degrees of freedom placed in a serial kinematic chain. The four joints are actuated by pneumatic stepper motors. In its home position (Fig. 8.11), the robot (excluding needle and racks) measures $72 \mathrm{~mm} \times 51 \mathrm{~mm} \times 40 \mathrm{~mm}$.

Fig. 8.1 shows the different parts of the robot. The base is fixed and has a rack, on which the cart can slide back and forth over a distance of $160 \mathrm{~mm}$. The cart itself contains a curved rack, which the lifter uses to tilt itself upwards over an angle up to $47^{\circ}$. Likewise, the lifter has another curved rack on which the platform can tilt to the other side over an angle up to $38^{\circ}$. Finally, the platform drives a needle holder back and forth over a distance of $80 \mathrm{~mm}$, on which the needle itself is mounted to target a breast phantom. The phantom is placed on a simple platform next to the robot. Not included is the option of horizontal angulation, in order to make the robot as compact as possible. Sixteen pneumatic tubes guide air to the different chambers of the four motors. The mass of the Stormram 4 (without base) is $62 \mathrm{~g}$.

\subsubsection{Stepper motors}

Two different stepper motors have been developed for the Stormram 4: the T-26 linear motor, and the C-30 curved motor. The general mechanism is shown in Fig. 8.2. It shows a rack (purple) and two double-acting pistons (red, green). Each piston consists of two piston heads, with jaws on the inside that engage on the rack by means of a wedge mechanism. By pressurizing the four chambers with appropriate waveforms, the pistons push the rack step by step in the desired direction. 

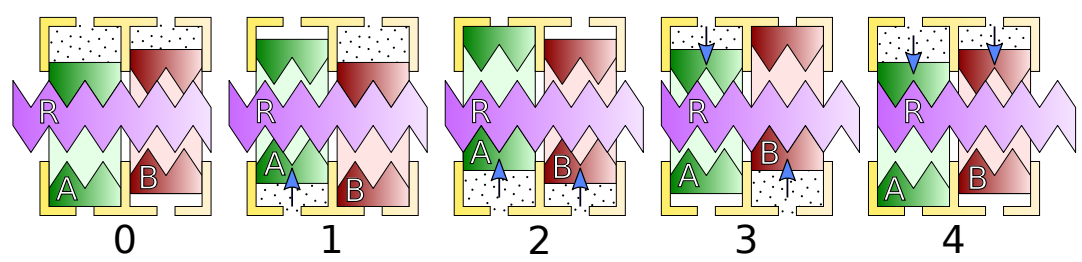

Figure 8.2: Sequence of states in two-cylinder linear motor. The pistons are labeled A and $\mathrm{B}$, the rack is labeled $\mathrm{R}$. The arrows indicate piston movements, causing step-wise movement of the rack to the right.

By design, the motor has zero backlash and nonzero hysteresis. The reason is that both pistons simultaneously push on the rack, but only one can be at its extreme position as can be seen in Fig. 8.2. The piston which is not at the extreme position, therefore acts as a wedge between the rack's teeth and the cylinder wall, which fixates the rack in its position resulting in zero backlash.

\section{Linear stepper motor}

The T-26 linear stepper motor is a miniaturization of the T-49 motor described in [51]. The T-26 measures $26 \mathrm{~mm} \times 21 \mathrm{~mm} \times 16 \mathrm{~mm}$. The two cylinders inside this motor have a square cross-sectional area of $10 \mathrm{~mm} \times 10 \mathrm{~mm}=100 \mathrm{~mm}^{2}$. The pistons act on a straight rack with teeth pitch $1.0 \mathrm{~mm}$ and teeth depth $1.2 \mathrm{~mm}$. The step size is $0.25 \mathrm{~mm}$, which is one-quarter of the pitch. The wedge ratio is $\frac{1.2}{0.5}=2.4$, so at a pressure of $0.4 \mathrm{MPa}$ the theoretical output force is $F=0.4 \cdot 10^{6} \cdot 100 \cdot 10^{-6} \cdot 2.4=96 \mathrm{~N}$, or $240 \mathrm{~N} / \mathrm{MPa}$. Due to friction in the seals and other sliding parts, the measured output force will be lower. If desired, the pressure can be increased to compensate for it and generate higher forces.

\section{Curved stepper motor}

The C-30 curved stepper motor is a novel design and measures $30 \mathrm{~mm} \times 23 \mathrm{~mm} \times 14 \mathrm{~mm}$ (excluding tube sockets). A 3-D rendering of the C-30 (without top cover) is shown in Fig. 8.3. The C-30 also houses two cylinders with the same square cross-sectional area of $100 \mathrm{~mm}^{2}$ as in the T-26 linear stepper motor. The difference is that the rack is not straight, but has a radius of curvature of $50 \mathrm{~mm}$. The teeth pitch size is $1.0^{\circ}$, which corresponds to an effective pitch distance of $0.87 \mathrm{~mm}$ on a circle with radius $50 \mathrm{~mm}$. The step size is $0.25^{\circ}$, which is one quarter of the pitch size. The teeth depth is $1.2 \mathrm{~mm}$. At a pressure of $0.2 \mathrm{MPa}$, the theoretical output torque is $M=$ $0.2 \cdot 10^{6} \cdot 100 \cdot 10^{-6} \cdot 1.2 \cdot 10^{-3} \cdot \frac{180}{0.5 \pi}=1.38 \mathrm{~N} \mathrm{~m}$, or $55 \mathrm{~N}$ at an arm length of $50 \mathrm{~mm}$. Similar to the T-26 motor, the measured output force will be lower 


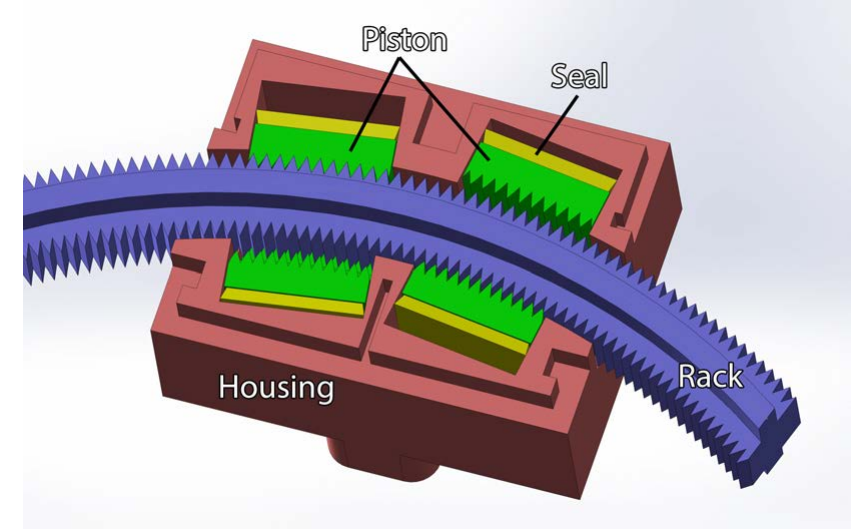

Figure 8.3: C-30 curved stepper motor with opened housing, exposing the rack, pistons and seals.

due to friction in the seals and other sliding parts, which can be compensated for by increasing the pressure.

The curved stepper motor has an axis of rotation. Therefore, a physical joint driven by this actuator can be combined with a passive pin joint with small radius, placed at the axis of rotation. This is useful to significantly increase the rigidity of the joint. In the linear stepper motor this would not be an option, as its axis of rotation is located at infinity.

\subsubsection{Production}

The parts of the Stormram 4 were printed with the Stratasys Objet Eden260 (Stratasys Ltd., Eden Prairie, MN, USA) in FullCure720 material. The seals were laser-cut from $0.5 \mathrm{~mm}$ thick silicone rubber. The motor housing and cover were glued together, and the sixteen polyurethane tubes were also glued into the sockets. The base was laser-cut from an $8 \mathrm{~mm}$ plate, engraved with grooves in which the linear rack and guide rail were glued.

\subsubsection{Controller}

The robot is controlled by a pneumatic valve manifold, shown in Fig. 8.4. The pneumatic routing for two valves connected to one motor is shown in Fig. 8.5. The eight valves are of type Festo MHA2-MS1H-5/2-2, and are controlled with an Arduino Mega board. Two different tube lengths were tested: $0.5 \mathrm{~m}$ and $5 \mathrm{~m}$.

A user interface allows to control the robot in different ways. The stepping frequency is controlled by one turn knob. In manual control mode, four other 


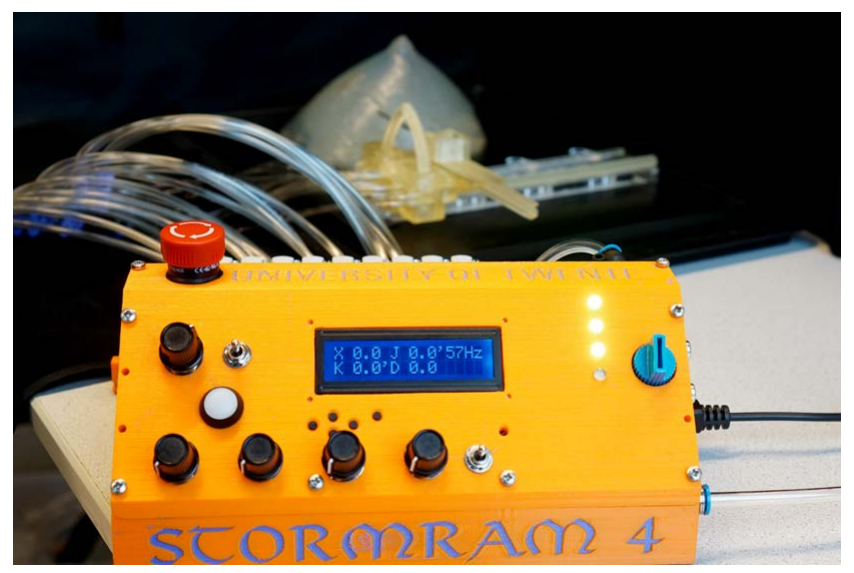

Figure 8.4: Computerized manifold.

turn knobs reflect the setpoints of the four joints to which the robot is steered. In automatic mode, the controller steers the robot through a pre-programmed sequence of setpoints instead.

The display shows the joint configuration vector or the needle tip position and orientation. Pushbuttons allow to cycle through different information panels and enable various actions such as calibration or automatic mode initiation. Voltage and pressure sensors examine operational status. Upon loss of pressure or voltage, the valves are switched off and the current joint coordinates are stored in memory.

\subsection{Kinematics and Workspace}

Fig. 8.6 shows a sequence of states during the needle alignment and insertion process. It shows the effect of actuation of three different joints, allowing to align and insert the needle in the $Y Z$-plane towards a given location.

Fig. 8.7 shows the kinematic configuration of the Stormram 4. Kinematically, it is a serial manipulator with four actuated joints. $J 1$ and $J 4$ are prismatic joints, driven by linear stepper motors. Joints $J 2$ and $J 3$ are rotational joints, driven by curved stepper motors. Point $E$ is the end-effector, physically the tip of the needle. Its coordinates are $\left(E_{x}, E_{y}, E_{z}, \phi\right)$, in which $\phi$ is the rotation angle around the $X$-axis, with $\phi=0^{\circ}$ being horizontal.

The joint configuration vector $\mathbf{v}=\left(\ell_{1}, \alpha, \beta, \ell_{4}\right)$ defines the pose of the full robot. The range of these variables are:

$$
\ell_{1}: 0 \mathrm{~mm} . .160 \mathrm{~mm}
$$


Ch 8: Design and Characterization of Stormram 4: An MRI-Compatible 146 Robotic System for Breast Biopsy

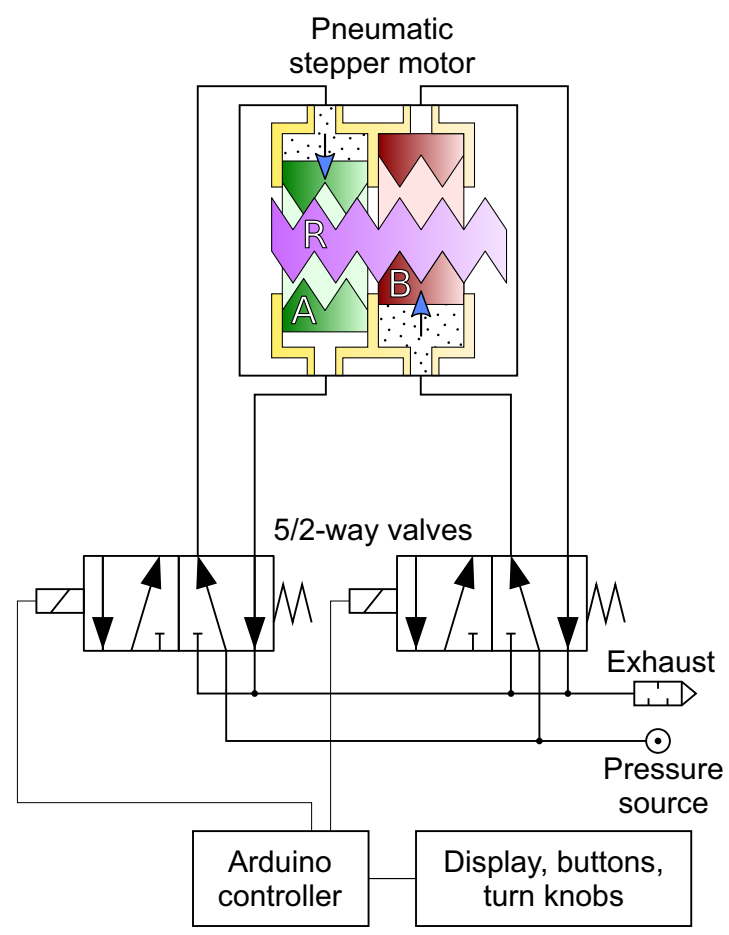

Figure 8.5: Pneumatic routing for one stepper motor. Two 5/2-way valves are connected to one motor, to pressurize the right chambers according to signals from the Arduino controller.

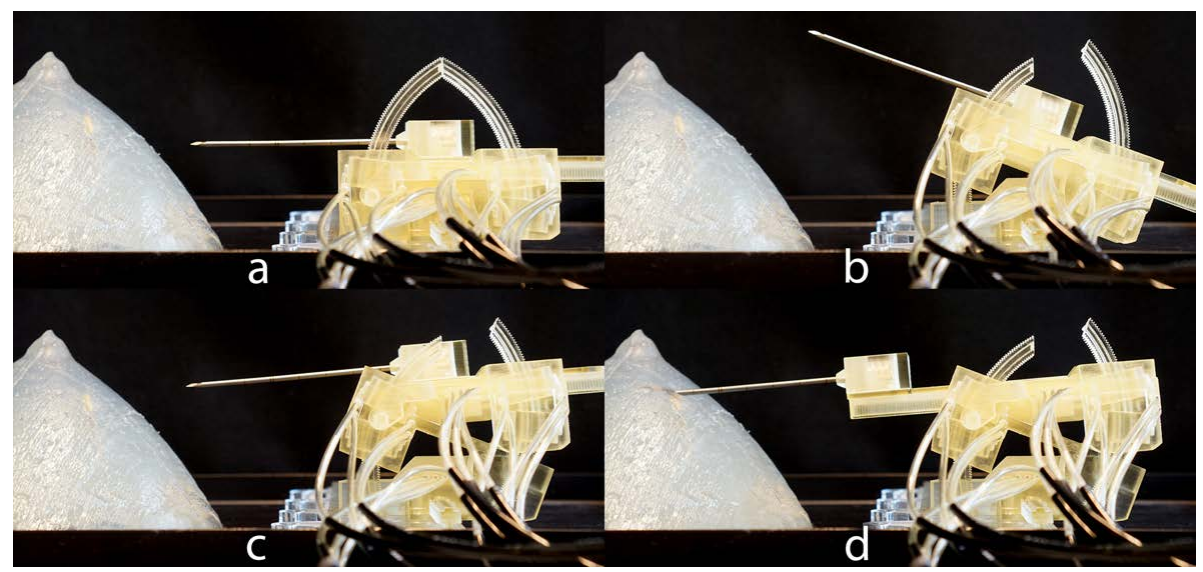

Figure 8.6: Four consecutive states in the needle alignment and insertion sequence. 


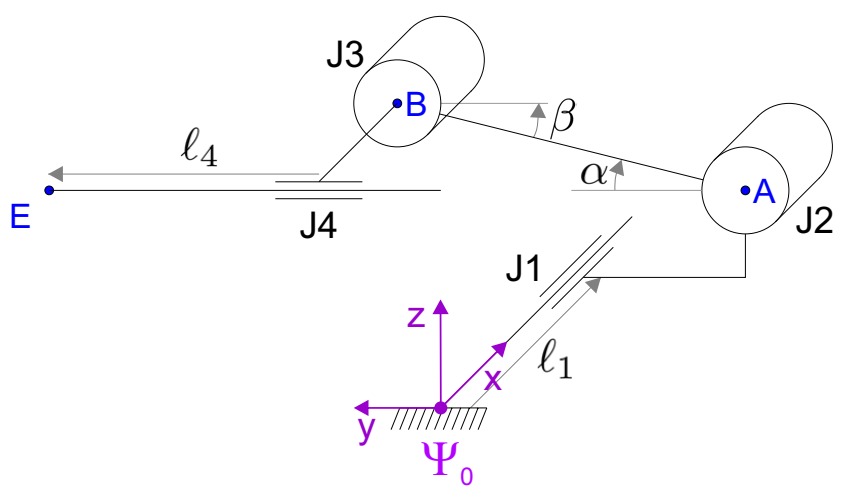

Figure 8.7: Kinematic configuration.

$$
\begin{aligned}
& \alpha: 0^{\circ} . .47^{\circ} \\
& \beta: 0^{\circ} . .38^{\circ} \\
& \ell_{4}: 65 \mathrm{~mm} . .145 \mathrm{~mm}
\end{aligned}
$$

The step size of $\ell_{1}$ and $\ell_{4}$ is $0.25 \mathrm{~mm}$, and the step size of $\alpha$ and $\beta$ is $0.25^{\circ}$.

In order to translate $\mathbf{v}$ to position and orientation of $E$ and vice-versa, forward and inverse kinematics are derived.

\subsubsection{Forward kinematics}

The end-effector coordinates are calculated as follows:

$$
\begin{aligned}
E_{x} & =\ell_{1} \\
E_{y} & =Y_{0}+W_{1} \cos \alpha-H_{2} \sin (\alpha-\beta)+\ell_{4} \cos (\alpha-\beta) \\
E_{z} & =H_{1}+W_{1} \sin \alpha+H_{2} \cos (\alpha-\beta)+\ell_{4} \sin (\alpha-\beta) \\
\phi & =\alpha-\beta
\end{aligned}
$$

The constants are:

$$
Y_{0}=-61 \mathrm{~mm}, H_{1}=15 \mathrm{~mm}, W_{1}=50 \mathrm{~mm}, H_{2}=18 \mathrm{~mm}
$$

\subsubsection{Inverse kinematics}

Given the desired end-effector location and orientation $\left(E_{x}, E_{y}, E_{z}, \phi\right)$, the joint vector $\mathbf{v}=\left(\ell_{1}, \alpha, \beta, \ell_{4}\right)$ was calculated. Geometrically, this is equivalent to 
Ch 8: Design and Characterization of Stormram 4: An MRI-Compatible 148 Robotic System for Breast Biopsy

one particular intersection of a line with a circle in the $Y Z$-plane. We first transformed $\left(E_{y}, E_{z}\right)$ to $\left(E_{y}^{\prime}, E_{z}^{\prime}\right)$, and then found an expression involving $\ell_{4}$ :

$$
\begin{aligned}
E_{y}^{\prime} & =E_{y}+H_{2} \sin \phi-Y_{0} \\
E_{z}^{\prime} & =E_{z}-H_{2} \cos \phi-H_{1} \\
W_{1}^{2} & =\left(E_{y}^{\prime}-\ell_{4} \cos \phi\right)^{2}+\left(E_{z}^{\prime}-\ell_{4} \sin \phi\right)^{2}
\end{aligned}
$$

Solving for $\ell_{4}$ and taking the solution with smallest value, leads to:

$$
\begin{aligned}
\ell_{1} & =E_{x} \\
\ell_{4} & =E_{y}^{\prime} \cos \phi+E_{z}^{\prime} \sin \phi \\
& -\sqrt{\left(E_{y}^{\prime} \cos \phi+E_{z}^{\prime} \sin \phi\right)^{2}-E_{y}^{\prime 2}-E_{z}^{\prime 2}+W_{1}^{2}} \\
\alpha & =\arcsin \frac{E_{z}^{\prime}-\ell_{4} \sin \phi}{W_{1}} \\
\beta & =\alpha-\phi
\end{aligned}
$$

The solution is valid if $\ell_{4}$ is real, and all parameters $\left(\ell_{1}, \alpha, \beta, \ell_{4}\right)$ are within the ranges.

Due to discretization of the stepper motors, the actual value of the four parameters must be a multiple of its step size $\left(0.25 \mathrm{~mm}\right.$ or $\left.0.25^{\circ}\right)$. The general approach is to round each parameter to its nearest reachable value, leading to end-effector positioning errors of the same order as the step size. This error could be reduced if the angle $\phi$ can be chosen: the optimal angle $\phi$ is the one (within its allowed range) which minimizes the end-effector positioning error.

\subsubsection{Workspace}

Fig. 8.8 shows the projection of the robot on the $Y Z$ plane in two different poses. One pose has configuration vector $\mathbf{v}=\left(0 \mathrm{~mm}, 47^{\circ}, 38^{\circ}, 65 \mathrm{~mm}\right)$. The red area is the projected reachable space of the end-effector $E$, and its area is calculated to be $140 \mathrm{~cm}^{2}$. The robot can move in the $X$-direction over a distance of $160 \mathrm{~mm}$ orthogonal to the $Y Z$ plane, resulting in a total workspace volume of $2.2 \mathrm{~L}$. 


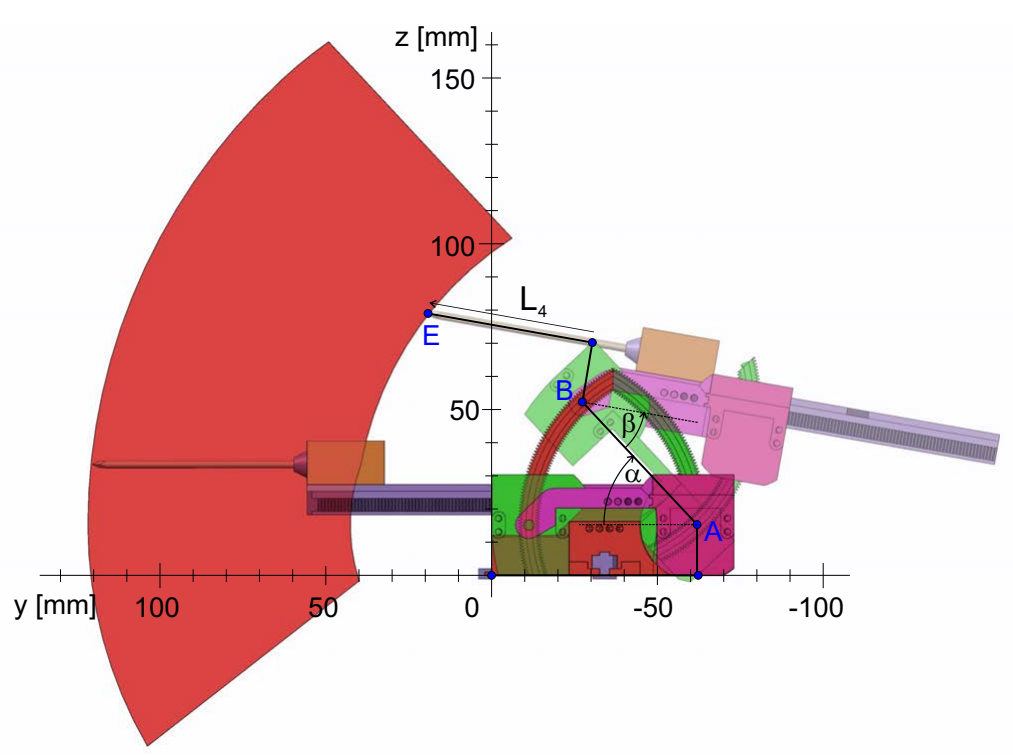

Figure 8.8: Workspace, and two extreme poses of the Stormram 4.

\subsection{Measurements and Results}

Performance measurements of the stepper motors and measurements on Stormram 4's positional accuracy have been performed to characterize the Stormram 4.

\subsubsection{Stepper motor force}

The T-26 linear stepper motor has been evaluated using a test bench as shown in Fig. 8.9. For a range of known masses, the minimum pressure on which the motor could lift the weight was recorded. The results are shown in Fig. 8.10. The highest measured force was $63 \mathrm{~N}$, at a pressure of $0.65 \mathrm{MPa}$.

It can be observed that the graph is approximately linear. Its slope is $103 \mathrm{~N} / \mathrm{MPa}$. This results in a mechanical efficiency of $43 \%$, when compared with the theoretical force slope of $240 \mathrm{~N} / \mathrm{MPa}$.

\subsubsection{Maximum operating speed}

The maximum operating speed (unloaded) was evaluated for all four joints of the Stormram 4 at a pressure of $0.25 \mathrm{MPa}$, by gradually increasing the stepping frequency until the motor misses steps. The experiment was performed with two different tube lengths, $0.5 \mathrm{~m}$ and $5 \mathrm{~m}$. The results are given in Table 8.1. 
Ch 8: Design and Characterization of Stormram 4: An MRI-Compatible

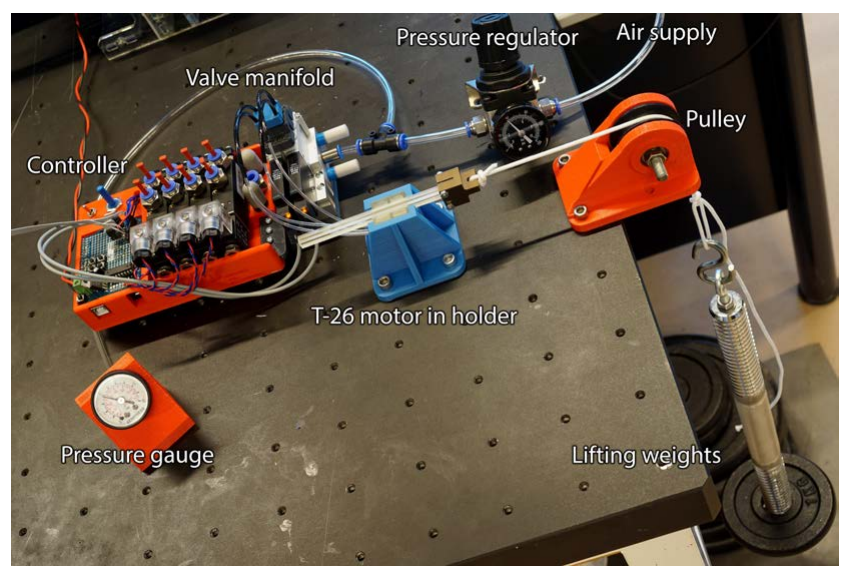

Figure 8.9: T-26 force measurement setup.

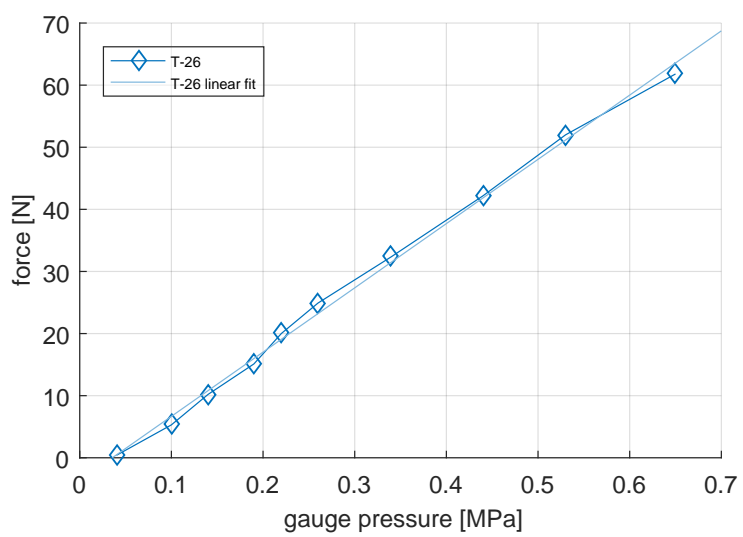

Figure 8.10: T-26 force versus pressure graph. 


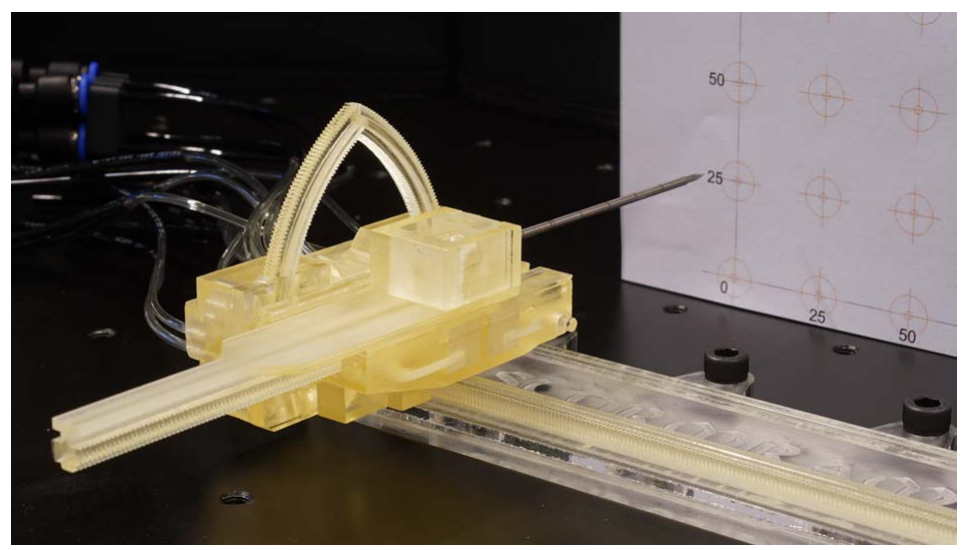

Figure 8.11: Accuracy measurement setup.

The motor of joint $J 4$ appears to have the lowest maximum frequency in both cases.

Under load, the maximum frequency is reduced to approximately $60 \mathrm{~Hz}$ for $0.5 \mathrm{~m}$ tubes, and $8 \mathrm{~Hz}$ for $5 \mathrm{~m}$ tubes. At these speeds, the maximum force is maintained for all joints, especially $J 4$, as required when inserting the needle into the phantom.

\subsubsection{Needle tip accuracy measurements}

The positional accuracy was evaluated using a sheet of paper positioned in the $Y=80 \mathrm{~mm}$ plane, as shown in Fig. 8.11. On this sheet, a $7 \times 5$ grid of targets with $25 \mathrm{~mm}$ spacing was drawn. The robot was programmed to move to these targets in succession, resulting in a series of punctures in the sheet. Afterwards, the $\mathrm{X}$ and $\mathrm{Z}$ offsets of each puncture (relative to its target) were measured.

For every value of $\mathrm{Z}$, the mean target-puncture offsets and the standard deviations were calculated for seven data points, and listed in Table 8.2. It can be observed that at higher $\mathrm{Z}$ values, there is an increasing bias in the $\mathrm{X}$ direction, resulting in a relative large standard variation of $0.71 \mathrm{~mm}$. But when

Table 8.1: Maximum unloaded motor stepping frequency.

\begin{tabular}{c|c|c} 
joint & freq at $0.5 \mathrm{~m}$ & freq at $5 \mathrm{~m}$ \\
\hline$J 1$ & $240 \mathrm{~Hz}$ & $65 \mathrm{~Hz}$ \\
$J 2$ & $240 \mathrm{~Hz}$ & $50 \mathrm{~Hz}$ \\
$J 3$ & $190 \mathrm{~Hz}$ & $50 \mathrm{~Hz}$ \\
$J 4$ & $160 \mathrm{~Hz}$ & $30 \mathrm{~Hz}$
\end{tabular}


Ch 8: Design and Characterization of Stormram 4: An MRI-Compatible

Table 8.2: Mean offset $\mu$ and standard deviation $\sigma$ in $\mathrm{X}$ and $\mathrm{Z}$ directions.

\begin{tabular}{c|c|c|c|c}
$\mathrm{Z}[\mathrm{mm}]$ & $\mu_{X}[\mathrm{~mm}]$ & $\sigma_{X}[\mathrm{~mm}]$ & $\mu_{Z}[\mathrm{~mm}]$ & $\sigma_{Z}[\mathrm{~mm}]$ \\
\hline 25 & -0.49 & 0.08 & -0.04 & 0.15 \\
50 & -0.03 & 0.09 & -0.10 & 0.16 \\
75 & 0.40 & 0.03 & 0.14 & 0.08 \\
100 & 0.96 & 0.10 & 0.33 & 0.13 \\
125 & 1.46 & 0.16 & 0.14 & 0.18 \\
\hline all & 0.46 & 0.71 & 0.10 & 0.21
\end{tabular}

Table 8.3: Comparison of T-26 with T-49. Note: two different housings have been developed for the T-49; the cylindrical one was used in the Stormram 3.

\begin{tabular}{c|c|c} 
Property & T-26 (Stormram 4) & T-49 (Stormram 3) \\
\hline Dimensions & $26 \times 21 \times 16 \mathrm{~mm}$ & $\varnothing 56 \times 40 / 49 \times 40 \times 31 \mathrm{~mm}$ \\
Volume & $8.7 \mathrm{~cm}^{3}$ & $99 \mathrm{~cm}^{3} / 61 \mathrm{~cm}^{3}$ \\
Force at 0.4 MPa & $38 \mathrm{~N}$ & $96 \mathrm{~N}$ \\
Step size & $0.25 \mathrm{~mm}$ & $1.0 \mathrm{~mm}$ \\
Max freq at $0.5 \mathrm{~m}$ & $240 \mathrm{~Hz}$ (unloaded) & $300 \mathrm{~Hz}$ (unloaded) \\
Max freq at $5 \mathrm{~m}$ & $30 \mathrm{~Hz}$ (unloaded), & $18 \mathrm{~Hz}$ (unloaded) \\
& $8 \mathrm{~Hz}$ (loaded) & $7 \mathrm{~Hz}$ (loaded)
\end{tabular}

this systematic offset is taken into account, the standard deviation reduces to $0.20 \mathrm{~mm}$. From the size of the punctures, the accuracy in Y direction is estimated to be $0.2 \mathrm{~mm}$.

A repeatability experiment was performed by executing the same travel path again. For all 35 targets, the needle reached the same spot as in the first run, so no new punctures were created. This can also be observed in the supplementary video. Considering the size of the puncture (average diameter $0.2 \mathrm{~mm}$ ), the repeatability is therefore better than $0.1 \mathrm{~mm}$.

\subsection{Discussion}

For comparison with the previous version, the properties of the current Stormram 4's T-26 and the T-49 stepper motor of the Stormram 3 are listed in Table 8.3. The T- 26 has less than half the strength of the T- 49 , but the dimension has been reduced approximately a factor two in all directions and the step size is reduced by a factor four.

The maximum frequency was found to be $160-240 \mathrm{~Hz}$ when $0.5 \mathrm{~m}$ tubes are used. In a MRI setting, $5 \mathrm{~m}$ long tubes are required which limits the maximum frequency to $30-60 \mathrm{~Hz}$ (unloaded) or $8 \mathrm{~Hz}$ (maximum force). Joint $J 4$ was found to have the lowest maximum frequencies, presumably due to higher friction 
and/or air leakage in the motor. The step size is $0.25 \mathrm{~mm}$ for the linear motors, so the minimum achievable speed is $2.0 \mathrm{~mm} / \mathrm{s}$ for $5 \mathrm{~m}$ tubes when maximum force is needed. If necessary, this minimum speed could be increased by using a larger step size of $0.5 \mathrm{~mm}$, which doubles the travel speed at the cost of reduced accuracy. A different solution would be to drive a single joint with two motors, to achieve both high speed and high precision.

The positional accuracy of the Stormram 4 is $0.71 \mathrm{~mm}$ in $\mathrm{X}$ direction, $0.21 \mathrm{~mm}$ in $\mathrm{Z}$ direction and approximately $0.2 \mathrm{~mm}$ in $\mathrm{Y}$ direction. This shows that sub-millimeter precision is achieved, which is a significant improvement over the Stormram 3. An important reason is the simplicity of the kinematic design: there are only four joints, and as all of these are directly actuated, the kinematic chain is fully free of backlash.

There is a systematic bias in $\mathrm{X}$ direction up to $1.0 \mathrm{~mm}$, likely due to manufacturing inaccuracies. If this bias is compensated for, by e.g. more precise manufacturing and/or calibration, the accuracy can be further improved to $0.17 \mathrm{~mm}$ in both $\mathrm{X}$ and $\mathrm{Z}$ directions. In future iterations of the robot, the structural rigidity could be improved by e.g. duplicating the $J 2$ and $J 3$ joints to the other side of the robot.

\subsection{Conclusion}

The Stormram 4 has demonstrated that it is able to manipulate a needle towards targets with sub-millimeter precision and a measured insertion force of approximately $40 \mathrm{~N}$. The actuators are free of backlash. In a MRI setting, $5 \mathrm{~m}$ long tubes are required and the maximum linear movement speed under maximum load is $2.0 \mathrm{~mm} / \mathrm{s}$.

The linear and curved motors are significantly smaller with a size of 26 and $30 \mathrm{~mm}$, than the state-of-art motors, and these are efficiently integrated in the different parts of the robot. The novel curved motor has shown that it can actuate a revolute joint with high precision. Due to the serial kinematic chain the number of moving parts are reduced to an absolute minimum, resulting in a design that is compacter than state-of-art robots.

Further tests on breast phantoms in a MRI scanner will be performed to simulate targeting lesions and also take tissue deformations into account. A breast fixation system and a biopsy firing mechanism are improvements to implement the full MRI-guided biopsy procedure in the design. As a proof-ofconcept, the Stormram 4 has shown that it is a suitable system to implement in clinical breast biopsy procedures. 
Ch 8: Design and Characterization of Stormram 4: An MRI-Compatible 154 Robotic System for Breast Biopsy

\section{Acknowledgments}

The authors would like to thank Gerben te Riet o/g Scholten for 3-D printing the different parts of the Stormram 4. 
CHAPTER 9

\section{Stormram 4: An MR Safe Robotic System for BREAST BIOPSY}

V. Groenhuis, F. J. F. Siepel, J. Veltman, J. K. J. van Zandwijk, and S. Stramigioli. Stormram 4: An MR Safe Robotic System for Breast Biopsy. Annals of Biomedical Engineering, 46(10):1686-1696, 2018. doi: 10.1007/s10439-018-2051-5 


\subsection{Abstract}

Suspicious lesions in the breast that are only visible on magnetic resonance imaging (MRI) need to be biopsied under MR guidance with high accuracy and efficiency for accurate diagnosis. The aim of this study is to present a novel robotic system, the Stormram 4, and to perform preclinical tests in an MRI environment. Excluding racks and needle, its dimensions are $72 \times 51 \times 40$ $\mathrm{mm}$. The Stormram 4 is driven by two linear and two curved pneumatic stepper motors. The linear motor is capable of exerting $63 \mathrm{~N}$ of force at a pressure of $0.65 \mathrm{MPa}$. In an MRI environment the maximum observed stepping frequency is $30 \mathrm{~Hz}$ (unloaded), or $8 \mathrm{~Hz}$ when full force is needed. The Stormram 4's mean positioning error is $0.73+-0.47 \mathrm{~mm}$ in free air, and $1.29 \pm 0.59 \mathrm{~mm}$ when targeting breast phantoms in MRI. Excluding the off-the-shelf needle, the robot is inherently MR safe. The robot is able to accurately target lesions under MRI guidance, reducing tissue damage and risk of false negatives. These results are promising for clinical experiments, improving the quality of healthcare in the field of MRI-guided breast biopsies.

\subsection{Introduction}

\subsubsection{Clinical challenge}

Breast cancer is one of the most frequently diagnosed cancer types in women, with an estimated 1.67 million new cases in 2012 [127]. It is essential to detect cancer at an early stage to optimize patient outcomes. Mammography is the primary imaging modality in screening programs, followed by ultrasound. MRI is often used in women with an increased risk for breast cancer. Lesions that are not visible on mammogram or ultrasound may be detectable on MRI which has a higher sensitivity than the other two imaging modalities[103]. CT (computed tomography) is not routinely used in breast cancer screening due to the additional radiation dose and the limited added value besides mammography. When a suspicious lesion is found and diagnosis needs to be confirmed the radiologist may decide to acquire a tissue sample through a biopsy.

The next step in the evaluation of MRI detected suspicious lesions is targeted ultrasound imaging and, if positive, ultrasound guided biopsy. However, the radiologist is often not capable to find the detected lesions on MRI during ultrasound scanning. This makes an MRI-guided biopsy necessary. It is difficult to target the lesion precisely during this procedure due to bore accessibility constraints that require the patient to be moved in and out of the scanner multiple times. When a positioning grid is used, discretization errors up to $4 \mathrm{~mm}$ are introduced[81]. In addition, aspiration and other unintentional body movements may cause breast tissue displacements. These factors may 


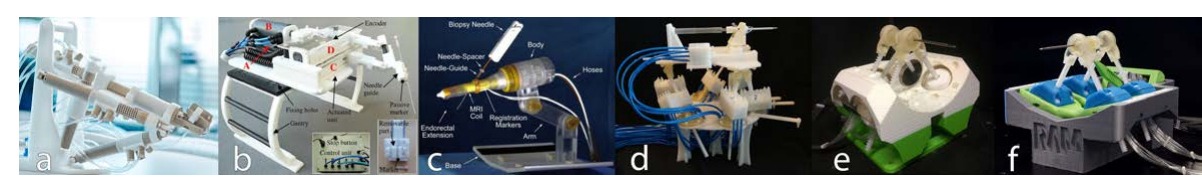

Figure 9.1: State-of-art pneumatic robots: a. Transrectal prostate biopsy robot by Bosboom et al.[15], b. Liver tumor ablation robot by Franco et al.[41], c. Endorectal prostate biopsy robot by Stoianovici et al.[119], d. Stormram 1 by Groenhuis and Stramigioli[46], e. Stormram 2 by Groenhuis et al.[4, 49], f. Stormram 3 by Groenhuis et al.[51]

cause false negative results, or a prolonged procedure due to repositioning of the needle[29, 129]

An MR safe robotic system could solve the shortcomings of existing manual MRI-guided biopsy procedures. Such a system, when placed inside the scanner bore, allows biopsies to be performed with fast MR imaging feedback. We expect that due to the increased accuracy fewer samples will be needed, resulting in reduced tissue damage and shorter procedure times. In view of past developments on this topic the aim of the Stormram 4 project is to design and characterize a novel MR safe robotic system for breast biopsy with significant improvements over earlier designs, especially in terms of accuracy and workspace.

\section{MR classification}

The ASTM F 2503 standard defines three possible classifications for MRI devices: MR safe, MR conditional and MR unsafe. The MR safe classification is assigned to devices that exclusively consist of non-metallic, non-magnetic and non-conductive materials, and can be assigned based on a scientific rationale (i.e. material composition) rather than test data. In contrast, the MR conditional classification indicates that the device is only safe when used under certain tested conditions. Finally, the MR unsafe classification indicates that the device is known to pose hazards in all MRI environments. This new standard is designed to avoid confusion and errors originating from the older terminology (MR compatible/MR safe), which are thus to be avoided in new research.

\section{State of the art}

Several MRI robots have been described in literature. The use of conventional electromagnetic motors are ruled out due to interference with the magnetic field of the MRI scanner. Various alternative actuation methods have been investigated: hydraulics, piezo motors, ultrasonic motors, cable transmissions, MR-driven ferromagnetics, flexible fluidic actuators, air turbines, direct-acting pneumatic cylinders and pneumatic stepper motors. Actuation by pneumatic 
stepper motors offers several advantages over the other categories: pneumatic stepper motors are inherently MR safe, tolerant for small air leakages, clean in medical applications, can be controlled with conventional pneumatic manifolds and allow for feed-forward control methods. 9.1(a-f) shows six pneumatic MRI robots found in literature. (a) Bosboom et al. developed and tested an MR safe, remote controlled parallel manipulator for transrectal biopsy guidance[15]. It is driven by five stepper motors. Each motor contains a rod with a helical hole pattern on which four single-acting cylinders act alternatingly, resulting in a screw movement of the rod. The robot requires a median manipulation time of approximately 6 minutes to move the needle guide to the commanded position, which makes it relatively slow. (b) Franco et al. described a needleguiding robot for laser ablation of liver tumors[41]. The relatively large robot is operated by four direct-acting pneumatic cylinders for which a special control scheme was developed to drive the piston accurately to the target position. Position feedback is acquired using electronic optical encoders, making it relatively complex and not MR safe. Its reported mean in-plane targeting error is $2.9 \mathrm{~mm}$. (c) Stoianovici et al. developed an MR safe robot for endorectal prostate biopsy, driven by three pneumatic rotational stepper motors called PneuStep[118, 119]. It uses fiber optic quadrature encoders for position measurements. The reported needle targeting accuracy is $0.37 \mathrm{~mm}$ in bench test and $2.09 \mathrm{~mm}$ in MRI. Being specifically designed for prostate biopsies the actuated workspace of this type of robot is too limited for breast biopsy purposes. The authors of this paper developed three earlier versions of the Stormram breast biopsy robot. Figure 1(d) shows the Stormram 1 which is driven by seven pneumatic linear stepper motors of which six form a Stewart platform and the seventh one inserts the needle longitudinally[46]. While this robot is able to demonstrate the proof-of-principle of pneumatic stepper actuation, the whole robot is too large to fit alongside a patient in the MRI scanner. Figure 1(e) shows the Stormram 2, which is driven by smaller stepper motors integrated inside ball joints, resulting in a more compact robot. Measurements have shown that it is able to target lesions in a phantom breast with a relatively poor accuracy of $6.0 \pm 2.0 \mathrm{~mm}[4,49]$, mainly due to clearances in the joints. 9.1(f) shows the Stormram 3 with a similar size and kinematic design as its predecessor. While the accuracy was improved to $2 \mathrm{~mm}$ and a stronger needle insertion actuator delivering up to $70 \mathrm{~N}$ of force was installed[51], the parallel kinematic structure resulted in a complex control structure and suboptimal workspace.

\section{Approach}

This paper describes the design and evaluation of the Stormram 4, shown in 9.2. It was developed to address the shortcomings of the state-of-art MRI robots, specifically in terms of size, complexity, accuracy and workspace[51]. 


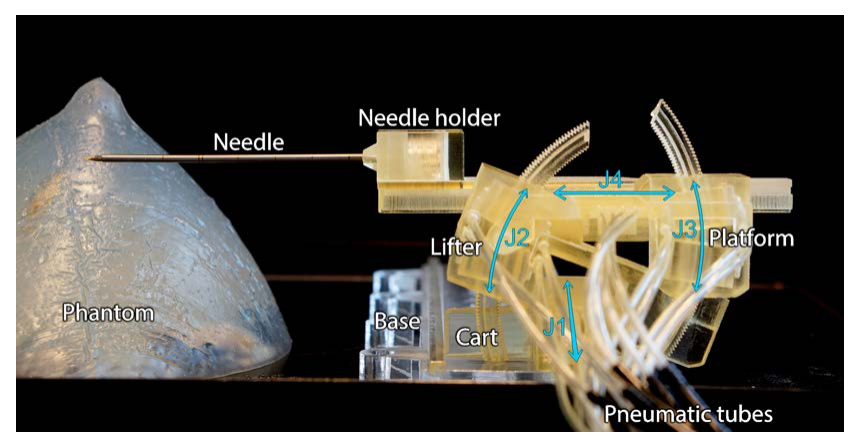

Figure 9.2: Stormram 4, with labeled parts and joints J1-J4.

The approach is to use a serial kinematic chain driven by a combination of linear and curved pneumatic stepper motors.

The use of a serial kinematic chain instead of a parallel one results in an enlarged workspace. High accuracy is achieved by using backlash-free joints in combination with a linear step size of $0.25 \mathrm{~mm}$, and an angular step size of $0.25^{\circ}$ (corresponding with $0.44 \mathrm{~mm}$ displacement at a radius of $100 \mathrm{~mm}$ ). Good stiffness is achieved by making the robot compact and by driving revolute joints at a radius of $50 \mathrm{~mm}$ from its axis. Flexible, thin $(2 \mathrm{~mm})$ pneumatic tubes are used in the proximity of the robot to allow unconstrained movement of the different degrees of freedom. Besides bench tests, extensive MRI tests are reported. For operator-independent, precise validation of the needle placement an automated needle detection algorithm was developed.

\subsection{Materials and Methods}

\subsubsection{Kinematic design}

The Stormram 4 is a serial kinematic manipulator with four degrees of freedom, each driven by a pneumatic stepper motor. In its home position the robot (excluding needle and racks) measures $72 \times 51 \times 40 \mathrm{~mm}$. The different parts of the robot are labeled in Figure 2. The base is fixed and consists of a linear rail over which the cart can slide back and forth over a distance of $160 \mathrm{~mm}$, this joint $\mathrm{J} 1$ is driven by a linear stepper motor. The base and lifter are linked by a revolute joint $\mathrm{J} 2$ which is driven by a curved stepper motor with a range of $47^{\circ}$. The lifter and platform are linked by another revolute joint J3 which is also driven by a curved stepper motor with a range of $38^{\circ}$. These two revolute joints combined allows the platform to move up and down and tilt vertically (but not horizontally). Finally, a linear stepper motor in the platform drives the needle holder longitudinally over a distance of $80 \mathrm{~mm}$, forming joint $\mathrm{J} 4$. 


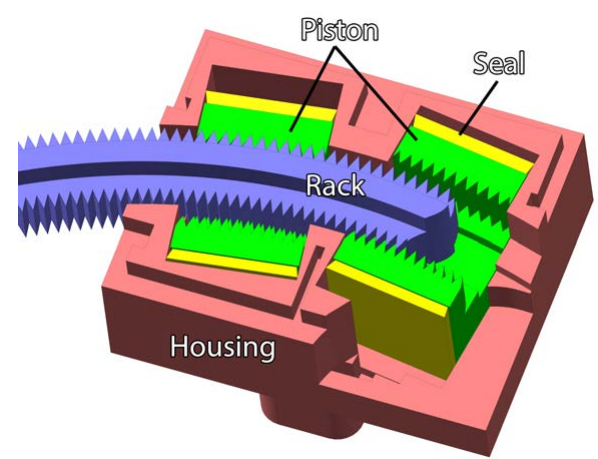

Figure 9.3: Cutaway view of the C-30 curved stepper motor, which consists of the housing, two pistons, four seals, four pneumatic tubes and the curved rack.

\subsubsection{Pneumatic stepper motors}

Two different stepper motors have been developed for the Stormram 4. The T-26 linear motor actuates a prismatic joint while the C-30 curved motor is designed to drive a revolute joint. Both motors are constructed and assembled similarly; the main difference is the radius of curvature for the rack which in turn influences the geometries of the housing and pistons.

Figure 9.3 shows the internals of the C-30 curved motor. Each of the two pistons (green) is operated by delivering pressurized air into either end of the cylinder, pushing the piston up and down. Silicone seals prevent leakage of air along the pistons. A piston has two jaws (series of teeth) on the inside that engage on the rack by means of a wedge mechanism, pushing the rack to the left or right in small steps. As the rack is curved with a radius of $50 \mathrm{~mm}$ there exists a well-defined rotation axis which can be supported by a hinge joint, significantly increasing stiffness of the mechanism.

\subsubsection{Production}

The majority of the robot parts were printed on an Objet Eden260 (Stratasys Ltd., Eden Prairie, MN, USA) in FullCure720 material and assembled together by gluing. The seals were laser-cut from $0.5 \mathrm{~mm}$ silicone rubber. Acrylic rods with a thickness of $3 \mathrm{~mm}$ were used in the two hinge joints between the cart, lifter and platform components located at points A and B in Figure 4. These rods were lubricated with silicone grease to allow rotational motion with sufficiently low friction and without any measurable play. Polyurethane tubes deliver pressurized air from the valve manifold to the robot. Silicone grease serves as lubricant for the moving parts. 


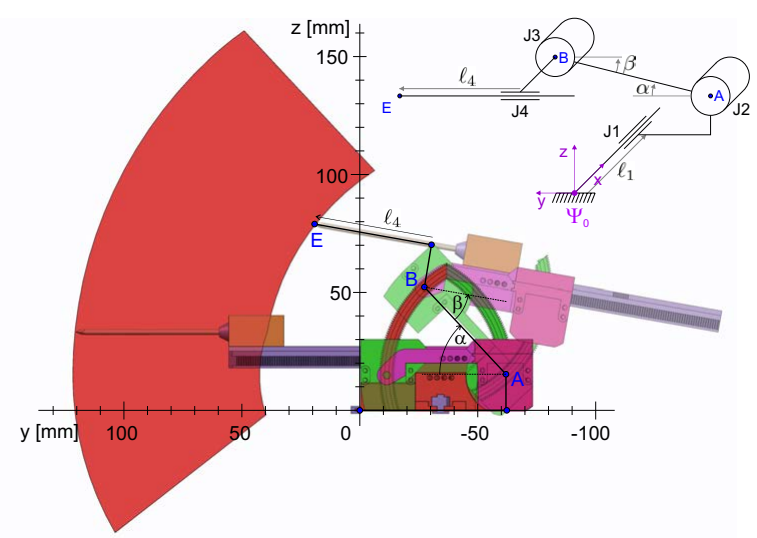

Figure 9.4: Workspace (red region), and two extreme poses of the Stormram 4. Top-right: kinematic configuration.

In this study the robot was equipped with an MR conditional Bard $14 \mathrm{G}$ $100 \mathrm{~mm}$ titanium needle (Bard Inc., Murray Hill, NJ, USA). The diamondshaped symmetric tip and needle rigidity resist bending due to needle-tissue interactions so that the needle can be assumed straight in all experiments. As the needle can be swapped with another one, e.g. to meet the conditions of a specific MRI scanner, the chosen needle is not considered to be an integral part of the robot. Based on the full material composition of the robot (excluding needle) the robot itself can be classified as MR safe. The robot base consists of a laser-cut acrylic plate on which a linear rack and a guide rail are positioned. The base itself is attached to a table printed in PLA (polylactic acid) in which ten fish oil capsules are embedded as fiducials for registration and alignment purposes.

Several breast phantoms were manufactured by pouring a hot mixture of PVC and plasticizer into a 3D printed mould generated from a mathematically described breast shape[3, 49]. In each phantom four to ten lesions were added (size range 5-20 mm) to the volume during the cool-off process. During this process the phantom solidifies to an elastic mass with randomly distributed lesions. For the lesions, either fish oil capsules or pieces of stiff PVC were used, both of which are relatively stiff and well distinguishable on MRI in certain sequences.

\subsubsection{Pneumatic controller}

The robot is controlled by a pneumatic valve manifold with user interface. Figure 9.5 shows a schematic of the controller including one pair of valves for 


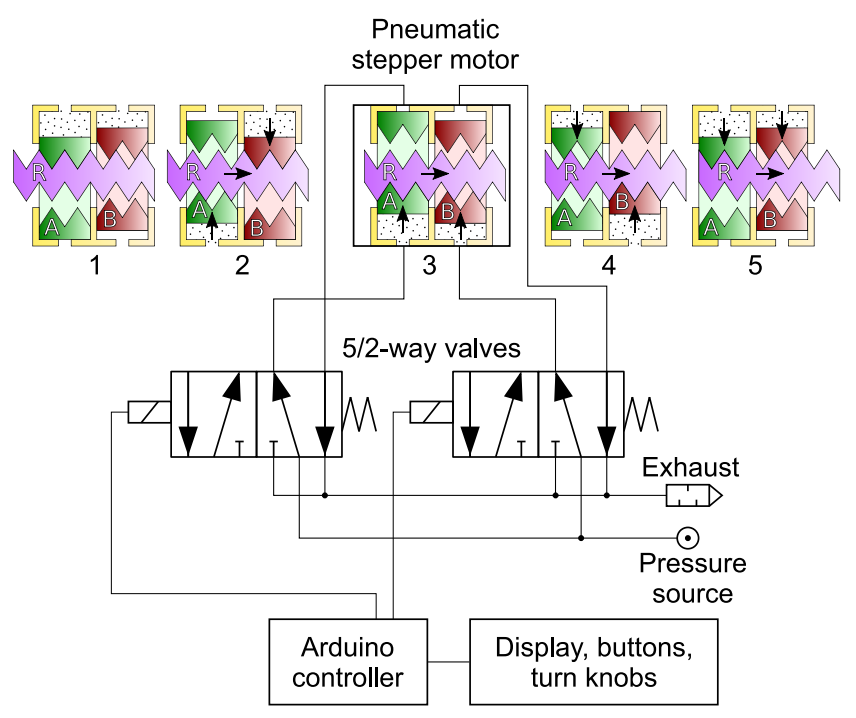

Figure 9.5: Pneumatic control schematic and five consecutive states of a single stepper motor.

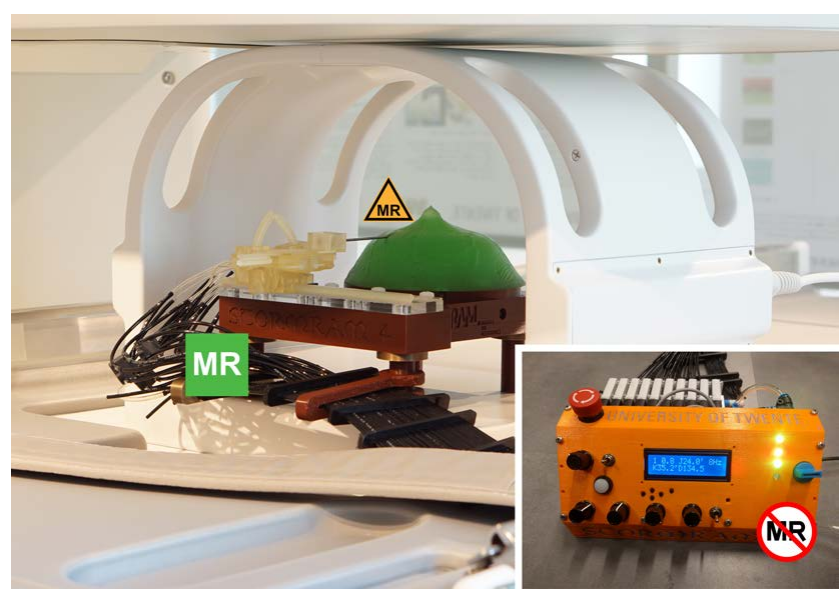

Figure 9.6: Experimental setup for MRI tests with an MR safe Stormram 4 piercing a breast phantom using an MR conditional needle. Bottom-right: MR unsafe pneumatic controller with user interface. 
driving one stepper motor. A photo of the actual controller can be seen in Figure 9.6 (bottom-right). Each of the four stepper motors is independently operated by a pair of 5/2-way valves of type Festo MHA2-MS1H-5/2-2 (Festo AG \& Co. KG, Esslingen, Germany) connected with $5 \mathrm{~m}$ long pneumatic tubes. This allows the MR unsafe controller to be placed outside the Faraday cage of the MRI to eliminate the possibility of RF interference and safety issues.

The pneumatic connection scheme and operating sequence of a single stepper motor is shown in Figure 9.5. Two pistons (green and red) act on a rack (purple). The five states, numbered 1-5, show the consecutive positions of the pistons and rack when it is operated from left to right. Exactly one piston can be fully engaged on the rack. This is always the piston that moved formerly and independent of the direction of load. The piston that moved most recently acts as a wedge between the rack and the cylinder housing, effectively eliminating any backlash in the motor by design. Reversal of direction results in a small hysteresis effect which could be exploited to achieve some form of sub-stepping. Alternatively, this effect could be avoided by choosing one particular direction of approach.

The sequence of valve states is controlled by an Arduino controller which maintains a memory of the current motor position for each of the four joints: (L1, $\alpha, \beta, \mathrm{L} 4)$. L1 is the displacement of joint $\mathrm{J} 1 \mathrm{in} \mathrm{mm} . \alpha$ is the angular displacement of joint $\mathrm{J} 2$ in degrees. $\beta$ is the angular displacement of joint $\mathrm{J} 3$ in degrees. L4 is the linear displacement of joint $\mathrm{J} 4 \mathrm{in} \mathrm{mm}$.

When one motor is commanded to move to a new position its valves are operated for the required number of steps at a given stepping frequency. As no position sensors are used, the control strategy is purely feed-forward. Correctness of the actual position is guaranteed when no steps are skipped since the last calibration, which can be assured by providing sufficient force or torque to perform the steps, exceeding the maximum loads on each actuator by a sufficiently large safety margin. Additionally, position feedback by MRI is possible.

The maximum stepping frequency is restricted by the length and diameter of the tubes, the cylinder stroke volume, the valve's airflow and switching speed. Up to a certain threshold frequency the performance of the motor is approximately constant; above the threshold frequency the force drops gradually until it does not move anymore at all.

\subsubsection{Kinematics and workspace}

Figure 9.4 shows the workspace and two different configurations of the Stormram 4 projected on the Y-Z plane. Using the two revolute joints and the linear needle insertion joint, the needle tip can reach targets within the red-colored region. The linear joint in the base allows movement of the robot in the $\mathrm{X}$ direction, resulting in a total workspace volume of $2.2 \mathrm{~L}$. If necessary, this volume could be increased further by elongation of the linear racks or the needle. 
Due to the serial kinematic chain, derivation of forward kinematics is straightforward. From angles $\alpha$ and $\beta$, length L4 and knowledge of the robot geometry, the Y- and Z- coordinates of the needle tip E can be calculated directly. The $\mathrm{X}$-coordinate follows directly from the displacement L1 of the linear joint in the base.

Inverse kinematics involves derivation of the four joint coordinates (L1, $\alpha, \beta, \mathrm{L} 4)$, given the target $(\mathrm{X}, \mathrm{Y}, \mathrm{Z})$ coordinates of the needle tip E. The displacement L1 of the linear joint in the base follows directly from the $\mathrm{X}$ coordinate, leaving us with derivation of $\alpha, \beta$ and L4 from a given point $\mathrm{E}$ in Figure 9.4. It can be noted that there are multiple solutions in general, as there are three variables and only two constraints. The needle insertion angle $(\alpha-$ $\beta$ ) can be chosen as the free parameter, upon the radiologist' discretion to e.g. circumvent delicate structures in the breast. The values of $\alpha, \beta$ and L 4 can then be found by solving the resulting set of equations. Existence of a unique solution depends on the feasibility of the different constraints - in particular the range of motions for the different joints. To help the radiologist or operator in choosing a suitable needle insertion angle its minimum and maximum values for a given position of $\mathrm{E}$ can be calculated and displayed on an interface from which a value can be picked somewhere in between.

Stepper motors can only reach a series of discrete states. The step size for linear motors is $0.25 \mathrm{~mm}$, while for rotational motors it is $0.25^{\circ}$. When the nearest reachable joint position is used, the resulting discretization error in $\mathrm{X}$-direction is up to $0.13 \mathrm{~mm}$, while for $\mathrm{Y}$ - and Z-directions the error is up to $0.39 \mathrm{~mm}$ with a fully extended needle.

\subsubsection{Segmentation and registration}

The LPS (left-posterior-superior) coordinate system is used in DICOM images of MRI scans, with the origin being at the magnet's isocenter. In contrast, the robot uses the XYZ coordinate system with the origin at a different site and the XY plane parallel to the MRI's coronal plane. The associated coordinate transformation is defined by the locations of ten fiducials, shown in Figure 7. Seven fiducials are upright, while three lay flat.

An automatic fiducial registration method is used to save time in the procedure. First, the ten fiducials are extracted based on the total volumes of the connected components in the geometrically corrected and binarized scan. From its sizes in posterior-anterior direction the three flat fiducials are identified and its mean position calculated from which the posterior-anterior component of the robot coordinate frame in MRI is defined. The remaining seven fiducials are registered based on intra-marker distances and, from the locations of its centroids, a best fit rigid 2D transformation in the coronal plane is constructed. Combined with the posterior component the $3 \mathrm{D}$ coordinate transformation is now fully defined. The mean registration error was measured to be $0.2 \mathrm{~mm}$ 


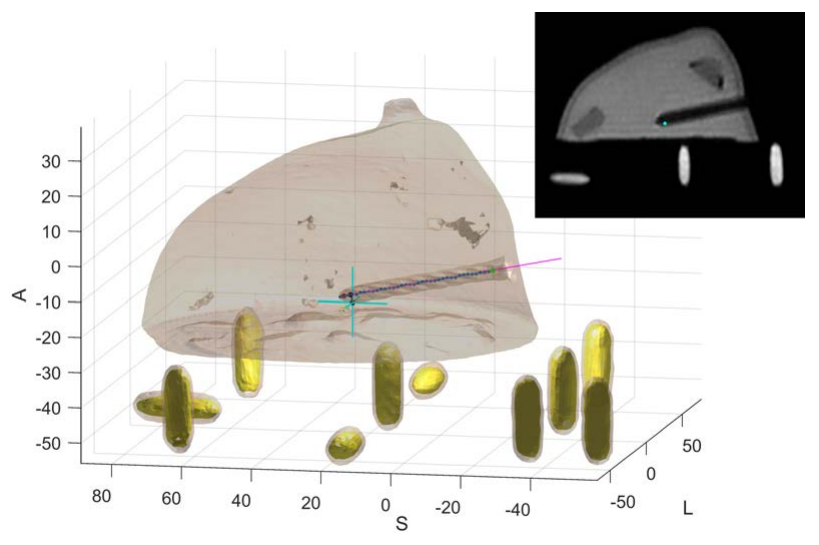

Figure 9.7: Segmentation of phantom with registration fiducials (yellow), target site (cyan), reconstructed needle segments (blue dots), best linear fit of needle centreline (magenta) and targeting error (black). Top-right: Sagittal MRI slice with target site (cyan).

- one order of magnitude smaller than the acquisition resolution of the MRI scans.

To evaluate the accuracy of the Stormram 4 in an MRI environment the needle must be localized on the confirmation scan. In order to utilize as much voxel information as possible to enable sub-pixel localization accuracy and to save operator time this is also performed automatically. This algorithm is based on finding connected dark voxels in the binarized image. From these voxels a tree graph is constructed based on the shortest distance from the image border. After some further processing involving traversing this tree graph it is possible to recognize the needle based on its shape involving with relatively constant cross-sectional area.

\subsubsection{Experiments}

Four experiments were conducted to characterize the force, speed and accuracy of the Stormram 4.

\section{Stepper motor force}

The maximum force of the T-26 stepper motor at low stepping frequency $(i 1$ $\mathrm{Hz}$ ) depends on the operating pressure. The relationship was studied by lifting a series of weights with known mass and finding the minimum required pressure such that it is just able to lift the weight. The schematic setup is shown in Figure 9.8. A linear fit is derived from the resulting pressure-force graph. 


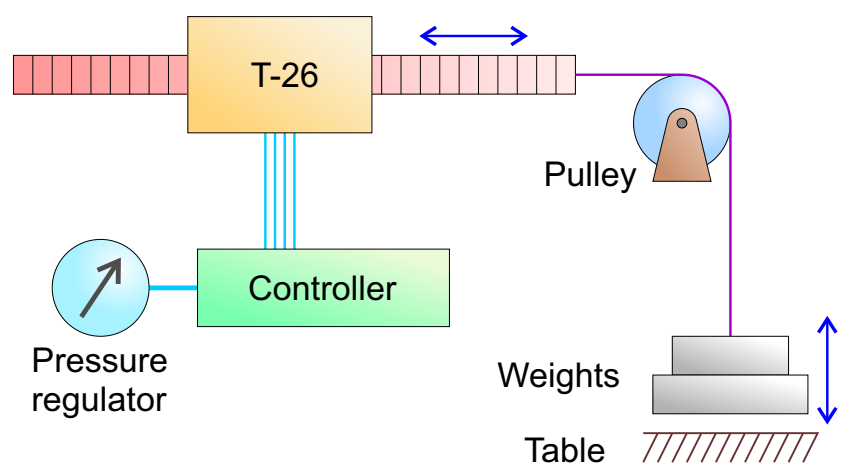

Figure 9.8: Force measurement setup. The T-26 stepper motor lifts weights of known mass, while the system pressure is adjusted using the regulator.

\section{Maximum stepping frequency}

The use of $5 \mathrm{~m}$ long tubes limits the stepping frequencies of the four motors. The maximum stepper motor frequencies for which each motor is just able to drive its joint without missing steps is recorded at a pressure of $0.25 \mathrm{MPa}$. For comparison, both short $(0.5 \mathrm{~m})$ and long $(5 \mathrm{~m})$ tube lengths were used in the test. Next, the maximum frequency of a motor under load with $5 \mathrm{~m}$ long tubes was derived by pulling a weight with the T-26 motor at increasing stepping frequency until a drop in the delivered force is observed.

\section{Needle tip accuracy in free air}

The accuracy and precision of the needle tip in free air were evaluated by piercing 30 targets drawn as crosshairs on a vertically positioned board. For each target, the error is defined as the distance from its center to the pierced hole.

\section{MRI accuracy tests}

MRI accuracy tests were conducted on breast phantoms. Thirty different sites were identified and targeted by the needle. The coordinates of each site are given as target for the robot. The error, defined as the offset between original site location and reconstructed needle position in the robot coordinate frame, was measured for each site. Breast deformations were not taken into account in this test. The Stormram 4 does not have a system to immobilize the breast, so the phantom on the table was allowed to deform freely resulting from needletissue interactions.

Figure 9.6 shows the experimental setup, with the Stormram 4 and phantom positioned inside a $0.25 \mathrm{~T}$ (G-Scan, Esaote SpA, Genoa, Italy) scanner. Taking 
into consideration the space requirements of the setup an abdominal coil with maximum internal height, width and length of $270 \mathrm{~mm}, 405 \mathrm{~mm}$ and $280 \mathrm{~mm}$ was used. The controller was placed outside the Faraday cage of the MRI scanner connected to the robot by a total of 30 tubes with a length of 5 metres. Of these 30 tubes, sixteen are used for actuating the four stepper motors while the remaining ones are reserved for future use such as the firing system of a biopsy gun.

The MRI scanner was geometrically calibrated using a custom 3D grid pattern before conducting the needle insertion experiments. From the measured pattern a 5th order correction function was defined to map observed (deformed) MRI coordinates to world coordinates. With this geometric correction, the registration error is reduced from up to $2 \mathrm{~mm}$ to an average of $0.2 \mathrm{~mm}$ within a cube-shaped volume with dimensions $180 \times 180 \times 180 \mathrm{~mm}$, centered at the magnet's isocenter. The correction function was applied to all observed coordinates in the tests. A 3D balanced steady-state free precession (bSSFP) sequence was used as the scanning protocol with parameters $\mathrm{TR}=10$ $\mathrm{ms}, \mathrm{TE}=5 \mathrm{~ms}$ and $\mathrm{FA}=60^{\circ}$. The scanning direction was the sagittal plane with a field-of-view of $240 \times 240 \mathrm{~mm}$ and acquisition matrix of $160 \times 160$ voxels, resulting in an acquisition resolution of $1.5 \times 1.5 \mathrm{~mm}$ in each slice. The acquired slice thickness was $2.0 \mathrm{~mm}$ and the (isotropic) reconstructed resolution was $0.94 \mathrm{~mm}$ in all directions. This scanning protocol is optimized for PVC lesions, as it shows good contrast between different tissue types in combination with a low signal-to-noise ratio.

\section{Testing procedure}

First, a pre-operative planning scan was performed. The resulting scan was segmented and the robot coordinate frame was defined. Next, a series of sites within the phantom were chosen. For each site, first the robot joint configuration vector was calculated using inverse kinematics. The free parameter representing the insertion approach angle was chosen as the midpoint of the range of possible values. The robot was operated to penetrate the needle in the phantom towards the selected site by manually rotating four turn knobs of the controller. First, the J1, J2 and J3 joint coordinates were adjusted to the pre-calculated position to align the needle with the target lesion. Next, joint $\mathrm{J} 4$ was adjusted to insert the needle longitudinally.

After reaching the target a confirmation scan was acquired. During this scan the joint configuration vector for the next target site was also calculated and, during reconstruction of the confirmation scan, the robot was already operated towards that next target in order to streamline actions as much as possible.

The confirmation scans were analyzed using an automated script to determine the needle position and angles from which the error distances were 


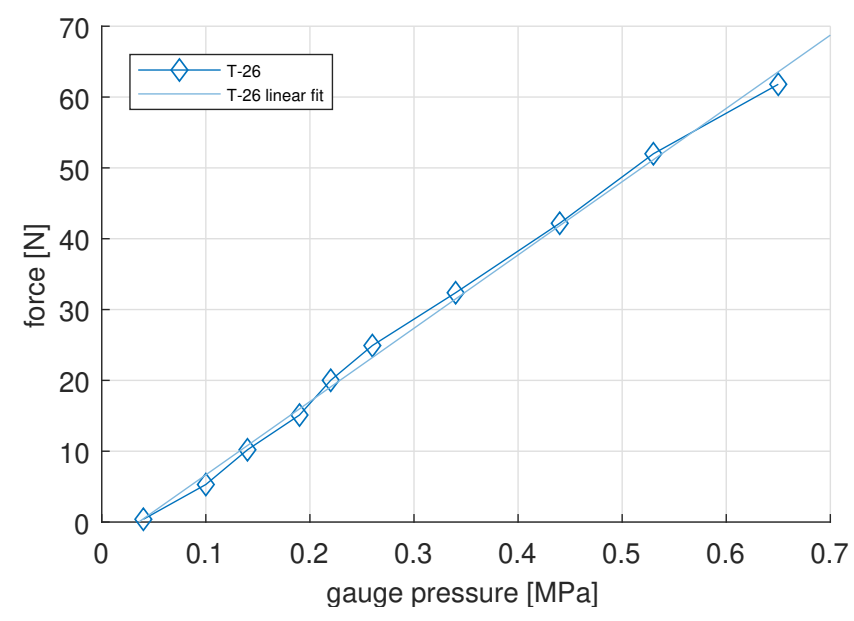

Figure 9.9: T-26 force versus pressure.

calculated. Whenever the needle detection algorithm was unable to correctly identify the needle or the human operator observed a discrepancy between reconstructed needle and MRI scan data, the needle was reconstructed manually using the software package 3D Slicer, version 4.7.

Although the Stormram 4 was equipped with a standard needle during the tests, it could be equipped with a biopsy gun when an appropriate biopsy needle is available. Samples taken by a biopsy gun are generally long and thin, typically $15 \mathrm{~mm}$ in length. This implies that in targeting small lesions accurate control of the needle depth is not critical. Hence, the shortest distance of the target site to the reconstructed needle, i.e. the normal distance error, is considered as the standard error measure[120].

\subsection{Results}

\subsubsection{Stepper motor force}

Ten data points were collected and plotted in Figure 9.9. The following linear relation was found between $F$, the measured force in Newton, and $P$, the supply pressure in Pascal:

$$
F=1.04 \cdot 10^{-4} P-3.7
$$

The highest recorded force was $63 \mathrm{~N}$ at a pressure of $0.65 \mathrm{MPa}$. At normal operating pressure of $0.25 \mathrm{MPa}$ the force is $22 \mathrm{~N}$. 


\begin{tabular}{l|l|l} 
Joint & Frequency at $0.5 \mathrm{~m}$ & Frequency at $5 \mathrm{~m}$ \\
\hline J1 & $240 \mathrm{~Hz}$ & $65 \mathrm{~Hz}$ \\
\hline J2 & $240 \mathrm{~Hz}$ & $50 \mathrm{~Hz}$ \\
\hline J3 & $190 \mathrm{~Hz}$ & $50 \mathrm{~Hz}$ \\
\hline $\mathrm{J} 4$ & $160 \mathrm{~Hz}$ & $30 \mathrm{~Hz}$
\end{tabular}

Table 9.1: Table 1: Maximum frequencies.

\begin{tabular}{l|l} 
2D error & $0.73 \pm 0.47 \mathrm{~mm}$ (range $0-1.71)$ \\
\hline $\mathrm{X}$ error & $0.46 \pm 0.70 \mathrm{~mm}$ (range $-0.63-1.71)$ \\
\hline $\mathrm{Z}$ error & $0.10 \pm 0.21 \mathrm{~mm}$ (range $-0.33-0.58)$
\end{tabular}

Table 9.2: Targeting error in free air.

\subsubsection{Maximum operating frequency}

The absolute maximum stepper motor frequencies of the four joints are listed in Table 9.1. For both short and long tubes joint J4 was found to be the limiting factor. In the case of $5 \mathrm{~m}$ long tubes the maximum stepping frequency was found to be approximately $30 \mathrm{~Hz}$. When loaded the maximum frequency was measured to be approximately $8 \mathrm{~Hz}$.

\subsubsection{Needle tip accuracy in free air}

Table 9.2 lists the observed targeting error when positioning the needle tip in free air as well as its decomposition in $\mathrm{X}$ and $\mathrm{Y}$ directions as measured by the positions of a series of 30 punctures in a vertically positioned sheet of paper with 30 marked targets. The resulting $2-\mathrm{D}$ error is $0.73 \mathrm{~mm}$. It can be observed that accuracy in Z-direction is approximately four times better than in X-direction. Closer examination of the error data revealed that needle tip movements in Z-direction are slightly skewed, i.e. these movements result in slight horizontal displacements as well.

\subsubsection{MRI accuracy tests}

A total of thirty locations in three different phantoms were selected and subsequently targeted.

Table 9.3 lists the error measures and other statistics for the measurement series. The full measurement data is available as a supplementary file.

In the last ten needle insertions the interval time for successive site targets was recorded and found to be 6:38 minutes - equal to the total time required for one MRI scan (including pre-scan calibration and image reconstruction). This scanning time was found to be the limiting factor in the needle insertion 


\begin{tabular}{l|l} 
Number of sites targeted & 30 \\
\hline Targeting interval (last 10 sites) & $6: 38$ minutes \\
\hline Needle segmentation method & 24 sites automatic, 6 sites manual \\
\hline Insertion depth & $25.0 \pm 11.5 \mathrm{~mm}$ (range $8.3-51.3)$ \\
\hline Apparent needle diameter & $5.7 \pm 0.8 \mathrm{~mm}$ (range $4.4-7.3)$ \\
\hline Normal distance error & $1.29 \pm 0.59 \mathrm{~mm}$ (range $0.43-2.63)$ \\
\hline 3D distance error & $1.87 \pm 0.80 \mathrm{~mm}$ (range $0.69-3.57)$ \\
\hline X error & $-0.37 \pm 0.87 \mathrm{~mm}$ (range $-2.11-1.43)$ \\
\hline Z error & $0.07 \pm 1.07 \mathrm{~mm}$ (range $-1.54-2.22)$ \\
\hline Depth error & $0.73 \pm 1.27 \mathrm{~mm}$ (range $-2.67-3.27)$ \\
\hline Azimuth angle error & $-0.25 \pm 1.16^{\circ}$ (range $\left.-2.78-2.93\right)$ \\
\hline Elevation angle error & $0.44 \pm 1.56^{\circ}$ (range $\left.-2.58-5.50\right)$
\end{tabular}

Table 9.3: MRI measurement statistics.

procedure as all other actions could be performed during the different phases of the scan.

The thirty confirmation scans were segmented and analyzed using an automated script. In six cases, the script did not correctly recognize the needle location due to insufficient penetration depth and/or due to the presence of air pockets near the targeting site. In these cases the needle location was determined manually by visual inspection of the confirmation scan using 3D Slicer software.

Figure 9.7 shows a rendering of the confirmation scan for target site 14 . The blue crosshair represents the commanded target and the needle is visible as a hole in the phantom with an apparent diameter of $5.3 \mathrm{~mm}$. The blue dots represent segments of the reconstructed needle. The magenta line represents a best linear fit of the needle centerline. For this particular target the normal error was measured to be $2.38 \mathrm{~mm}$.

Table 3 lists the error statistics in targeting all 30 sites. The average targeting error (shortest distance to needle) was found to be $1.29 \pm 0.59 \mathrm{~mm}$ (range $043-263)$. When insertion depth is also considered the resulting 3 -D error is $187 \pm 0.80 \mathrm{~mm}$ (range $069-357$ ).

The error measurements for the individual needle location components (X, $\mathrm{Z}$, depth, azimuth and elevation) reveal that the errors in lateral (X and $\mathrm{Z}$ ) directions are comparable. However, there is a significant bias present in insertion depth of $0.73 \mathrm{~mm}$ and also in elevation angle $\left(0.44^{\circ}\right)$. The deviations in the components are about three to six times the nominal step size of the linear and rotational joints. 


\subsection{Discussion}

\subsubsection{Comparison with state-of-art robots}

Compared with earlier robots in the Stormram line the newly developed iteration is a significant improvement in terms of workspace, accuracy, size and complexity. It is also smaller and faster than other state-of-art robots described in this paper. In terms of accuracy a fair comparison to those robots is difficult due to differences in stiffness of the targeting organ (prostate or liver) compared to that of the breast. Also, the resolution and calibration quality of the MRI scanner significantly affects the results. Comparing the Stormram 4 with the prostate robot by Stoianovici et al. shown in Figure 1(c) which has a reported accuracy of $0.37 \mathrm{~mm}$ in bench test and $2.09 \mathrm{~mm}$ in MRI, the Stormram 4 obtained slightly better results in MRI.

\subsubsection{Kinematics}

The choice to use a serial kinematic chain instead of a parallel manipulator has shown to have positive effects on size, complexity, accuracy and workspace. It has only four joints, which are all directly actuated. The revolute joints incorporate high stiffness thanks to the actuation method by curved stepper motors.

\subsubsection{Stepper motor force and frequency}

Measurements have shown that the T-26 motor can deliver $63 \mathrm{~N}$ of force at a pressure of $0.65 \mathrm{MPa}$. This force is approximately one order of magnitude higher than the minimum required force to pierce the breast skin with a sharp $14 \mathrm{G}$ needle (order of $10 \mathrm{~N}$ ). The maximum stepping frequency in an MRI environment is limited to $8 \mathrm{~Hz}$ when maximum force is needed, corresponding to a motion speed of $2 \mathrm{~mm} / \mathrm{s}$, or $2^{\circ} / \mathrm{s}$ for rotational joints, but it can be reliably increased to $20 \mathrm{~Hz}$ for unloaded movements.

The T-26, and its curved counterpart, the C-30, can actuate all joints of the robot without chance of missing steps, provided that the operating pressure and stepping frequency are appropriate for handling the specific robot loads. From a known initial position full knowledge of the joint state vector during normal operation can be guaranteed by feed-forward control.

\subsubsection{Stormram 4 accuracy}

Measurements have shown that the robot achieves an accuracy of $0.73 \pm 0.47$ $\mathrm{mm}$ in free air, but the accuracy and precision in X-direction are not as good as in Z-direction. It was found that movement in the Y-Z plane is not precisely 
perpendicular to the $\mathrm{X}$ axis, causing measurable horizontal needle displacements when the needle is tilted up or down. This can be attributed to deficits in structural stiffness of the kinematic design and, combined with clearances in both linear joints, these result in measurable parasitic motions. When these factors are taken into account the horizontal accuracy could be improved to 0.2 mm. A better solution would be to improve the mechanical design by adding structural strength and reducing clearances in the different joints and links.

In MRI, the targeting error was found to be $1.30 \pm 0.61 \mathrm{~mm}$ (range $0.44-$ $2.85 \mathrm{~mm}$ ). This error is larger than the accuracy in free air and can be mainly attributed to needle-tissue interactions which result in deflections of the needle. Other error sources are in imaging and registration: the used 3D bSSFP scanning sequence has an acquisition resolution of $1.5 \times 1.5 \times 2.0 \mathrm{~mm}$ and therefore relies on sub-pixel reconstruction accuracy for segmentation of both the fiducials and the needle shape. In a clinical setting the different field strengths and/or scanning sequences involved may result in different shapes of the needle artifacts, potentially resulting in higher or lower targeting errors. Lastly, a weak correlation between Z-position and X-error can be observed with correlation coefficient 0.5 , which is in accordance with measurements in free air.

\subsubsection{Procedure time}

The mean procedure time per site, involving robot manipulation and performing the confirmation scan, was measured to be $6: 38 \mathrm{~min}$. This is equal to the total time of a 3D bSSFP scan, including pre-scan calibration and image reconstruction. An MRI scanner with a stronger magnetic field (e.g. $3 \mathrm{~T}$ ) would allow quicker scans with the same SNR and resolution, potentially reducing the procedure time.

The robot needs less than 1:30 minutes to move the needle from one target site to another. This is mainly attributed to the needle insertion and retraction speed of $2 \mathrm{~mm} / \mathrm{s}$ over a distance of up to $80 \mathrm{~mm}$. When faster operation is desired it could be achieved by combining two stepper motors in a single linear joint with different step sizes, to allow both large and small steps to be made at the same stepping frequency of $8 \mathrm{~Hz}$ under load.

\subsubsection{Future developments}

Additional developments are needed before clinical trials can be considered. A breast fixation system integrated in a breast $\mathrm{RF}$ coil needs to be developed to immobilize a patient's breast. The robot is to be equipped with a biopsy gun in order to take tissue samples. The structural stiffness should be improved to consistently maintain high accuracy. Safety mechanisms and procedures need to be developed for sterilization and also to cope with any possible system 
failure. When all these elements are incorporated, the robotic system has good potential for in-vivo clinical use.

\subsection{Acknowledgments}

We thank Gerben te Riet o/g Scholten for 3-D printing and laser-cutting various parts of the Stormram 4 robot. 



\section{SunRAM 5: An MR SAFE ROBOTIC SYSTEM FOR BREAST BIOPSY, DRIVEN BY PNEUMATIC STEPPER MOTORS}

V. Groenhuis, J. Siepel, and S. Stramigioli. Sunram 5 : An MR safe robotic system for breast biopsy, driven by pneumatic stepper motors. In M. H. Abedin-Nasab, editor, Handbook of Robotic and Image-Guided Surgery, chapter 22, pages 375-396. Elsevier, 2019

Included with permission from Elsevier. 
Ch 10: Sunram 5: An MR safe robotic system for breast biopsy, driven by 176 pneumatic stepper motors

\subsection{Abstract}

Sunram 5 is the fifth generation MR safe robotic system for breast biopsy. It has five degrees of freedom and is driven by six linear and curved pneumatic stepper motors plus three singular cylinders, all constructed by rapid prototyping techniques. The design, production and evaluation of both single pneumatic cylinders and various types stepper motors are described in detail in this chapter. Control strategies are also discussed such as how multiple motors can work together in order to achieve both high speed and high accuracy, despite the relatively low stepping frequencies associated with long pneumatic lines between controller and motor. Sunram 5 also includes a breast fixation system, an emergency needle ejection mechanism and fast and precise needle insertions under near-realtime MRI guidance, giving potential to improve accuracy and efficiency in MRI-guided breast biopsy procedures.

\subsection{Introduction}

\subsubsection{Clinical challenge}

Breast cancer is the most commonly diagnosed cancer type among women [20]. Early detection is essential for a good prognosis. In many countries breast cancer screening programs have been set up. Mammography (x-ray) is the primary imaging modality, as it is quick and able to detect the majority of cancers. In addition, ultrasound (US), palpation, computed tomography (CT) and/or magnetic resonance imaging (MRI) may be used for more conclusive screening.

MRI has the highest sensitivity of all imaging modalities. It makes a 3D scan with (sub-)millimeter resolution with good contrast between different types of tissue. After inserting a contrast agent, areas of angiogenesis (local growth of veins) can be distinguished.

Certain patient groups may undergo MRI screening even if no abnormalities are found on mammography or ultrasound. Women carrying the BRCA 1 or 2 gene have an elevated risk for developing breast cancer and benefit from periodic MRI screening [8]. Additionally, women with unexplicable complaints in the breast (e.g. pain) may be advised to undergo MRI screening. Finally, MRI scans may also be useful for the doctor to prepare for breast surgeries.

When a suspicious lesion is found, a biopsy is required for accurate histological evaluation. This is first attempted under ultrasound guidance, which is a relatively easy procedure if the lesion is well visible on US. In this procedure the radiologist inserts a biopsy needle into the breast towards the lesion. When correctly positioned, the biopsy gun is fired, capturing a tissue sample of the suspicious lesion which is subsequently stored for pathology assessment. This 
method is the gold standard in determining malignancy of suspicious lesions.

In some cases the lesion is not visible on US, but only on MRI. An MR guided biopsy is then necessary. In this procedure the radiologist inserts a biopsy needle using a grid or post-pillar system. This is normally performed outside the MRI scanner due to accessibility constraints of the scanner bore. The patient has to be moved in and out of the scanner multiple times in the procedure. Small movements of the breast due to respiration and tensions may cause displacements of the lesion to be sampled, making it difficult to precisely target it. Also, the spacing of the grid introduces a discretization error when this method is used. To compensate for these errors, a relatively large needle (9 gauge, equivalent to $3.8 \mathrm{~mm}$ ) is generally used, and multiple tissue samples are acquired to obtain a high confidence in acquiring at least one tissue sample of the lesion. One example system is the vacuum-assisted breast biopsy (VABB) system by Hologic. Still, the confirmation scan may indicate an inadequate needle placement, requiring re-positioning of the needle, leading to additional tissue damage or a false negative biopsy.

In order to resolve the shortcomings of the manual MRI-guided breast biopsy procedure, the needle should not be inserted blindly outside the MRI scanner, but inside the scanner bore itself. This more or less implies the necessity of a robotic system to position, align and insert the biopsy needle due to accessiblity constraints. This eliminates the possibility of patient movements and allows for (near-)realtime imaging guidance during robotic needle insertion. Additionally, robotic systems potentially allow for more precise needle insertions. With such a system, a relatively thin (16 gauge, equivalent to $2.1 \mathrm{~mm}$ ) biopsy needle is sufficient, resulting in considerably less tissue damage compared to conventional systems such as the VABB.

\subsubsection{MRI compatibility of surgical robots}

An important requirement for devices inside the MRI scanner is that these are safe to use in the specific environment. The ASTM F2503 standard defines three categories of MRI devices: MR safe, MR conditional and MR unsafe [114]. The MR safe requirement implies that the device is free of metallic, ferromagnetic and conductive materials and therefore inherently safe to use in all MRI scanners. This is regardless of the field strength and other parameters such as maximum gradients and minimum distance to patient. The MR conditional classification indicates that the device is only safe when certain given conditions are all met, while devices with the MR unsafe classification pose unacceptable risks and cannot be used in any MRI environment. This scheme replaces the former one (MR compatible/safe) which is known to cause confusion and errors: many "MRI compatible" devices were only tested under certain conditions and sometimes resulted in unsafe behaviour in other environments, leading to serious risks. 
Ch 10: Sunram 5: An MR safe robotic system for breast biopsy, driven by 178 pneumatic stepper motors

\subsubsection{Actuation methods for MR safe/conditional robots}

The MR safe/conditional requirement implies that conventional electromagnetic motors cannot be directly used in actuation of any MR robot. Several alternative actuation methods have been proposed and demonstrated:

- Piezo motors and ultrasonic motors are electric motors that only cause limited interference with the MRI's magnetic field. Using dedicated control electronics and taking certain precautions, such motors may be classified MR conditional and may be usable in actuation of MRI robots $[70,123,125]$. A drawback is that piezo/ultrasonic motors cannot be classified MR safe due to the use of electricity and metallic materials, so the MRI safety and imaging quality aspects have to be re-evaluated each time when operating conditions are expanded.

- Bowden cables transport energy via solid wires guided through tubes $[24,70]$. Instead of tubes a system of pulleys can also be used. These techniques allow to place conventional motors away from the robot (outside the Faraday cage of the MRI scanner). If the wires and Bowden tubes (or pulleys) are made of non-metallic materials, the system could be made MR safe. Friction, backlash and elasticity in the rigid materials may make an effective energy transfer difficult, especially when many bends are present in the transmission line.

- Pneumatics use clean air as energy transfer medium which is abundant in hospitals and laboratory environments. As small leakages are acceptable, pneumatic cylinders can be manufactured using rapid prototyping techniques. Important limitations are the compressibility of the medium which makes precise position control of a single cylinder difficult $[41,135]$, and also the long distance between the (MR unsafe) controller manifold and robot leads to long pneumatic lines which results in relatively low bandwidth.

- Hydraulics make use of liquid to deliver power to the robotic system [78, 133]. The liquid is kept in a closed system with compressor and valves and leaks are to be avoided. A hydraulic device requires the use of precisely engineered components, which makes rapid prototyping relatively difficult compared to other techniques.

- Actuation by magnetic spheres driven by gradients of the MRI scanner have also been demonstrated [40]. This technique is relatively complicated as it requires precise control of the MRI's gradients while at the same time mitigating the imaging artifacts induced by the magnets.

- Shaped memory alloy (SMA) actuators generate unidirectional movements when heat is applied to a SMA spring. The heat can be generated 
by applying current through the SMA spring, of which the self-resistance results in resistive heating. Bidirectional movement is generated using complementary pairs of SMA springs [67]. The use of metallic materials in the SMA actuators and the application of current through it make the SMA actuators MR-conditional at best.

The authors of this chapter use pneumatics as the energy transfer method in the form of pneumatic stepper motors. We show that fast and precise control is possible despite the low bandwidth and lack of direct position feedback.

\subsubsection{State-of-art}
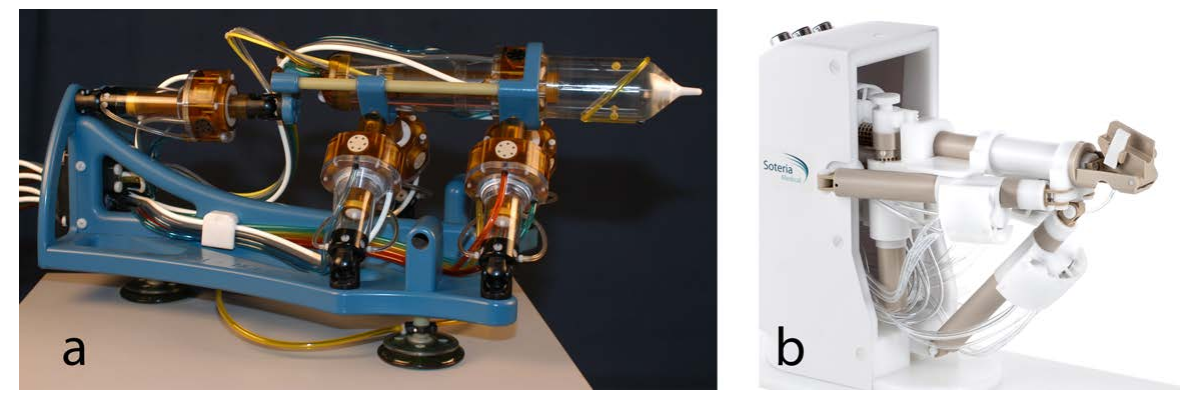

Figure 10.1: Two state-of-art MRI manipulators driven by pneumatic stepper motors. a) MrBot by Stoianovici et al. [120]. b) Soteria Remote Controlled Manipulator (RCM) by Bomers et al. [15].
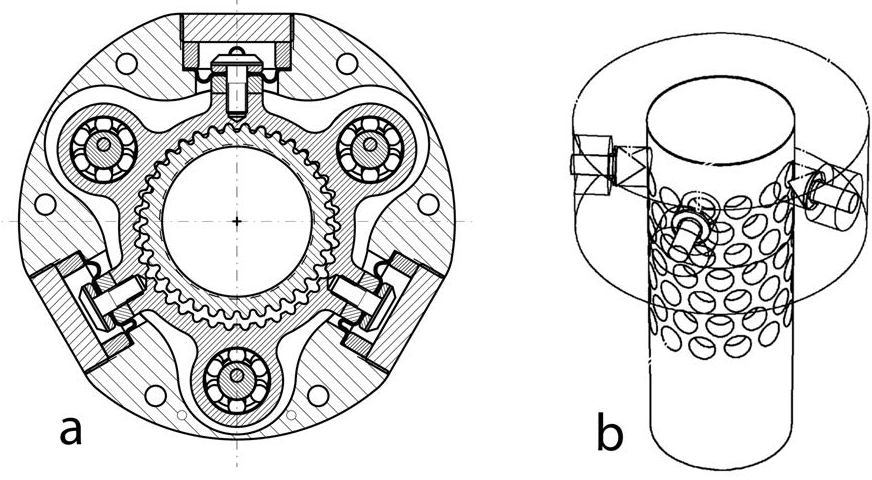

Figure 10.2: Pneumatic motors used to actuate the corresponding manipulators in Figure 10.1. a) PneuStep by Stoianovici et al. [118], b) Pneumatic stepper motor by Bomers et al. $[15]$ 
Ch 10: Sunram 5: An MR safe robotic system for breast biopsy, driven by 180 pneumatic stepper motors

Many MRI surgical robots have been developed in the past by various research groups. In this chapter a selection of robotic systems driven by pneumatic stepper motors is discussed: first three robots by other research groups and then five robots by the authors of this chapter.

\section{Pneumatic MRI robots by Stoianovici, Bomers and Sajima}

Stoianovici et al. developed several MRI robots for prostate biopsy. One example is the MrBot, shown in Figure 10.1(a) [120]. It is driven by six PneuStep rotational stepper motors of which a schematic cross section is shown in Figure 10.2(a). The PneuStep motor consists of three diaphragm cylinders that are connected to an internal gear. By alternatingly pressurizing the three cylinders, the internal gear is translated along a circular trajectory and its hoop gear in turn engages a spur gear. A leadscrew mechanism then converts the rotational motion of the spur gear into linear motion, resulting in movement of the robotic system. PneuStep makes use of optical positional encoders to detect and correct for missing steps, allowing to operate it at higher stepping speeds when less than maximum torque is needed. The valve manifold is put inside a shielded enclosure within the MRI room, allowing to reduce the tube lengths to a minimum [118].

The Soterial Remote Controlled Manipulator (RCM) by Bomers et al. is shown in Figure 10.1(b). Like MrBot, this robot is designed for prostate interventions [15]. It is driven by five pneumatic stepper motors of which a schematic drawing is shown in Figure 10.2(b). Its five cylinders have cone tips mounted on the pistons which engage on a two-dimensional pattern of holes on the rod. Pressurization of one cylinder pushes the associated cone tip into one hole, forcing the hole to align with the cone tip by the associated wedge mechanism and hereby introducing a displacement. Sequential pressurization of the right combination of cylinders result in either a screw movement or a linear movement of the rod, resulting in a small or large displacement of the robot linkages. The cylinders are double-acting, a single tube is used for the return stroke of all five pistons so that six tubes are used per actuator [15].

Sajima et al. developed a manipulator driven by rotational stepper motors [110]. Each stepper motor consists of three single-acting cylinders that act on a rotation gear by means of a wedge mechanism. By sequentially pressurizing the three cylinders the gear is driven around in either direction. In this design the gears have to be back-driveable in order to allow retraction of the pistons in the non-pressurized cylinders for continuous movements. A leadscrew finally converts the rotational motion of the gear into linear motion of the manipulator linkages.

An important limitation of the design of Sajima is that the wedge mechanism must be back-driveable due to the use of single-acting cylinders. This implies that the teeth cannot have sharp angles and significant torque is lost by 
friction of the sliding surfaces. On the other hand, Sajima's design is relatively compact and easy to manufacture compared to the designs of Stoianovici and Bomers.

The low nominal stepping frequency resulting from the long pneumatic tubes is an issue which has to be adressed in order to achieve both high speed and high accuracy. Stoinanovici's design utilizes position encoders which allow to speed up the motors when less than maximum torque is needed, while Bomers' design uses a 2-D hole pattern that enables both large and small actuation steps.

\section{Stormram 1-4 and Sunram 5}

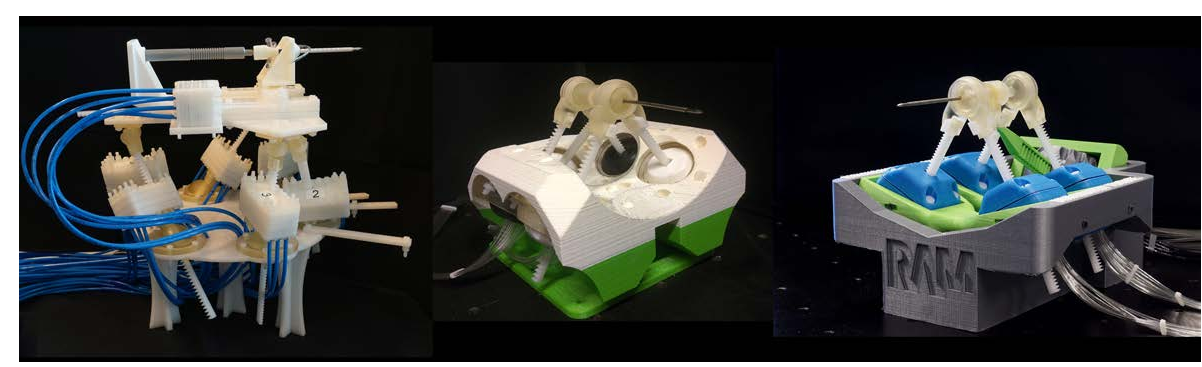

Figure 10.3: Stormram 1 (left), Stormram 2 (center) and Stormram 3 (right).

Five MR safe breast biopsy robots have been developed by the authors of this chapter. All of them are driven by pneumatic linear or curved stepper motors and rapid prototyped by 3D printing and laser-cutting techniques. Table 10.1 lists the main characteristics of each of the five generations.

Stormram 1 (Figure 10.3, left) was developed in 2014 and has seven degrees of freedom (DOFs) [46]. Its large size and small workspace makes it unsuitable for practical applications, so in 2015 the Stormram 2 was developed (Figure 10.3 , middle) with five degrees of freedom. This robot is driven by stepper motors integrated inside $45 \mathrm{~mm}$ ball joints for compactness [4]. In the next year Stormram 3 was developed (Figure 10.3, right), also with five degrees of freedom and with improved accuracy, force characteristics and workspace thanks to redesigned joints and smaller step sizes [51]. Still, the use of a parallel kinematic chain made control complicated and the low stepping frequency in an MRI environment made the robot too slow.

In 2017, the Stormram 4 was presented (Figure 10.4, left). This system has a serial kinematic manipulator with four degrees of freedom, of which two are driven by curved stepper motors and two by linear stepper motors. With a compact size of $72 \times 51 \times 40 \mathrm{~mm}$ (excluding racks) this robot combines a relatively large workspace with good accuracy, still at low speed in MRI environment 
Ch 10: Sunram 5: An MR safe robotic system for breast biopsy, driven by 182 pneumatic stepper motors

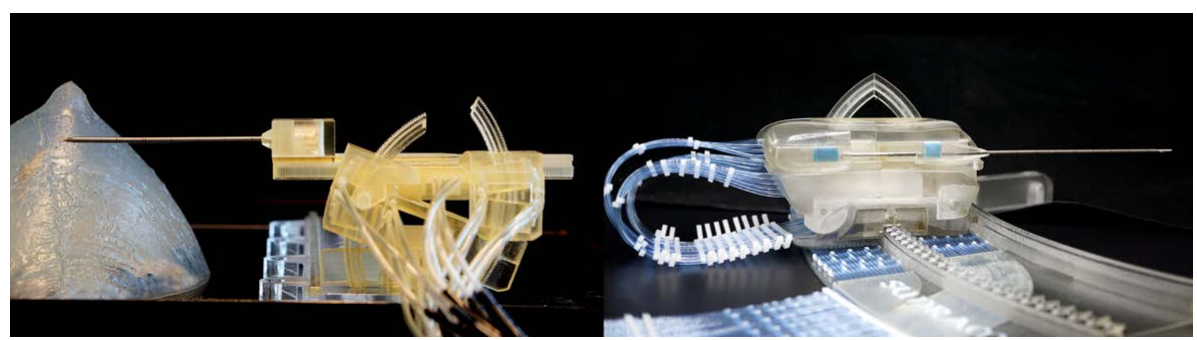

Figure 10.4: Stormram 4 (left) and Sunram 5 (right).

$[50,55]$. In the next year the Sunram 5 was developed (Figure 10.4, right) which utilizes dual-speed motors on certain axes. The Sunram 5 has five degrees of freedom driven by a total of six stepper motors and also includes a breast fixation system, a pneumatic biopsy gun and a safety needle ejection mechanism [54].

All stepper motors used in Stormram 1-4 and Sunram 5 consist of two or three double-acting cylinders that engage on a straight or curved rack by means of a wedge mechanism $[47,53]$. The rectangular-shaped cylinders allow efficient stacking of multiple cylinders within a single housing. This is particulary useful for the two dual-speed motors in Sunram 5 in which four or five cylinders are positioned in line, enabling both large-step and small-step movements in the same movement direction [52]. 


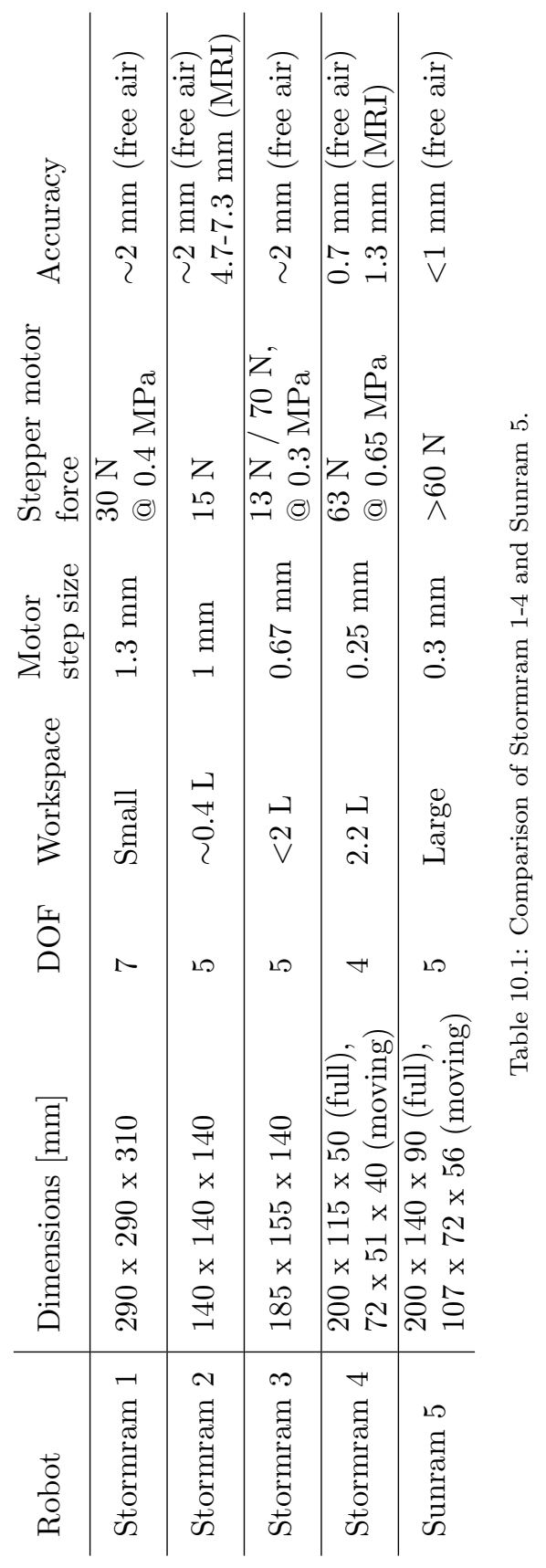


The minimum required number of degrees of freedom in a biopsy robot is three: the location of a lesion is represented as a point in space while the needle insertion direction can in principle be chosen arbitrary. Additional DOFs are useful in order to navigate around impassable structures: examples are the grating of the breast fixation system and any anatomical features indicated by the radiologist that should be avoided. With five degrees of freedom Sunram 5 has sufficient dexterity to circumvent such structures and reach difficult locations in the breast.

\subsubsection{Organization of the chapter}

The remaining sections of this chapter are organized as follows: Section 10.3 describes the design and manufacturization of single-acting cylinders, Section 10.4 describes linear and curved stepper motors and dual-speed motor concepts, Section 10.5 describes the design and kinematics of Sunram 5, Section 10.6 describes control methods for cylinders, stepper motors and robotic systems, Section 10.7 describes evaluation methods and experimental results of stepper motors and the Stormram 4, and Section 10.8 finally wraps up the chapter.

\subsection{Pneumatic cylinders}

This section describes the principles, design and production aspects of pneumatic cylinders. The single-acting cylinder is presented as an example as this is the easiest to design, manufacture and evaluate. The necessary files for 3-D printing and laser-cutting this device are available for download [45].

Pneumatic cylinders consist of a hollow cavity in which a piston can slide back and forth. When a pressure $P$ is applied in the cavity at one side of the piston, a force $F=P \cdot A$ is exerted on its surface $A$ which may result in motion, delivering work to the environment. By alternatingly applying pressure to either side of the piston through separate pneumatic connections, the piston can be moved back and forth pneumatically and this is called a double-acting cylinder.

\subsubsection{Rectangular cross-sectional shape}

Most traditional cylinders have a circular cross-section. The main reasons are that circles have an optimal area/circumference ratio, allowing to produce cylinders with relatively thin walls. Also, circular holes can be easily manufactured by conventional drilling techniques and the absence of sharp corners in the walls makes sealing relatively easy. On the other hand, cylinders with circular walls are much more difficult to manufacture precisely using 3D printing techniques than cylinders with straight walls due to characteristics involved 
in 3D printing. More specifically, the additive manufacturing techniques that deposit filament layer-by-layer result in staircase effects and poor surface finish at steep overhangs, present in horizontally-oriented cylindrical cavities. In vertically-oriented cylindrical cavities the layered structure also causes difficulties in motion and sealing. In order to circumvent these drawbacks, box-shaped cylinders are used in this chapter. Its cross-sectional area is rectangular and the structures can be manufactured with good accuracy. An additional advantage is that a rectangular cylinder makes more efficient usage of the available space than circular cylinders with the same wall thickness.

No protruding cylinder rods are used in the double-acting pneumatic cylinders. While it would be possible to use such rods with appropriate sealing, different methods are used to transfer work to the environment. In case of the stepper motors, interaction between pistons and racks occurs by means of teeth positioned inbetween the two heads of each piston.

\subsubsection{Sealing}

A piston moves inside a cylinder as a result of pressure being applied to one of its surfaces. This pressure is supplied by a pressure source through a valve manifold outside the MRI room. It is essential that pressure is transferred as effectively as possible, so leakage in the cylinder must be kept to a minimum in order to avoid pressure drops. Without proper sealing a significant amount of leakage would occur through the gap between the piston and the cylinder walls; this gap is necessarily present to allow sliding motion of the piston inside the cylinder.

Elastomer O-rings as used in circular cylinders do not function in rectangular cylinders due to the four right-angled corners which the O-ring cannot cover. Instead, plates of silicone rubber with thickness $0.5 \mathrm{~mm} . .1 .0 \mathrm{~mm}$ are chosen as the sealing material. A laser cutter is used to cut rectangular pieces out of it. Alternatively, the seals can be cut by hand using a cutting tool. The seal width and height should be approximately $0.2-0.3 \mathrm{~mm}$ larger than the respective cylinder cross-sectional dimensions to ensure good sealing without causing excessive friction.

The seal edges need to be slanted with angle $2^{\circ}-15^{\circ}$. This implies that the seal faces are not equal: one side has a larger surface area than the other. For effective sealing the larger face must be oriented towards the air chamber, while the smaller face touches the piston head. When pressurized air acts on the larger surface of the seal it effectively pushes the seal edges against the cylinder walls and this results in a proper sealing function.

In most cases it is not necessary to fixate the seals to pistons mechanically. The sufficient condition is that the piston is only moved pneumatically and not by external forces, which guarantees that the seals are pressed against the piston heads at all times and will not become detached. 
Ch 10: Sunram 5: An MR safe robotic system for breast biopsy, driven by 186 pneumatic stepper motors

\subsubsection{Design of single-acting cylinder}

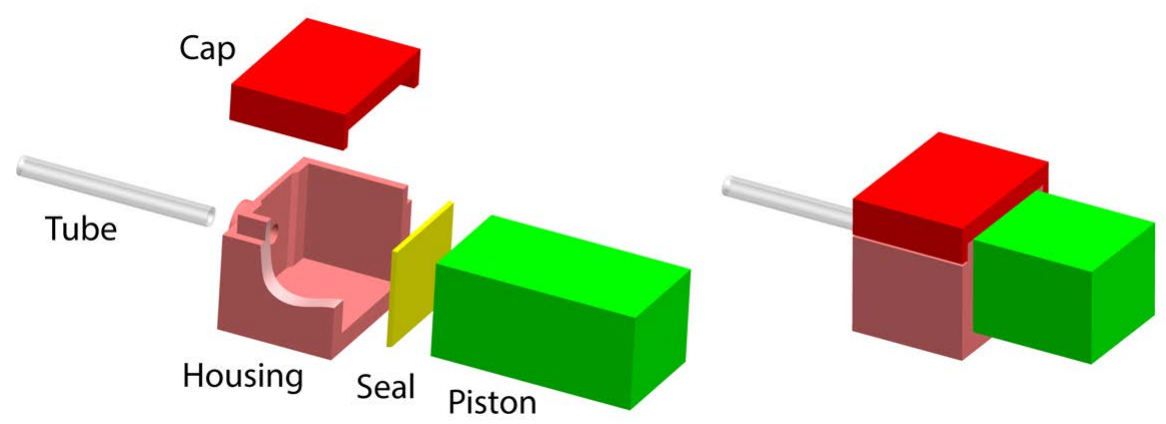

Figure 10.5: Exploded (left) and assembled (right) views of single-acting cylinder design, with housing (red), piston (green) and seal (yellow).

The development of a new pneumatic actuator using the technology described in this chapter is an iterative process. In order to study the dimensional aspects of the cylinder parts and how to seal it properly, it is useful to design and build the open single-acting cylinder shown in Figure 10.5. This design allows to explore different sealing techniques and part dimensions in order to obtain the right tolerances experimentally, as the piston and seal can be taken out even after gluing the housing. It also allows to evaluate the piston forces and air leakages at a range of pressures. The presented techniques can subsequently be applied to create rectangular-shaped cylinders of any size.

The shown cylinder has a cavity of $12 \times 10 \times 10 \mathrm{~mm}$ and walls of $2 \mathrm{~mm}$. This results in outer dimensions of $16 \times 14 \times 12 \mathrm{~mm}$, excluding the pneumatic socket which is a $2 \mathrm{~mm}$ hole with depth $4 \mathrm{~mm}$. The theoretical output force at a pressure of $0.4 \mathrm{MPa}$ is $\left(0.4 \cdot 10^{6} \mathrm{~Pa}\right) \cdot\left(12 \cdot 10^{-3} \cdot 10 \cdot 10^{-3} \mathrm{~m}^{2}\right)=48 \mathrm{~N}$.

The cylinder is printed in two parts: a housing and a cap. The piston has a flat head which covers the cross-sectional area with a small clearance (order of $0.1 \mathrm{~mm}$ ), to allow for smooth movements without wobbling. In Figure 10.5 a solid block is used as piston, but in practical applications the piston may include features to interact with the environment. The seal is also rectangular, but must be slightly larger than the cross-sectional area of the cylinder: typical dimensions are $12.3 \times 10.3 \times 0.5 \mathrm{~mm}$. The cross-sectional area can be reduced if friction is too high, or increased in case of (excessive) leakage. A thicker (e.g. 1 or $1.5 \mathrm{~mm}$ ) allows for more rigidity which may be useful in cylinders larger than $15 \mathrm{~mm}$ in size. 


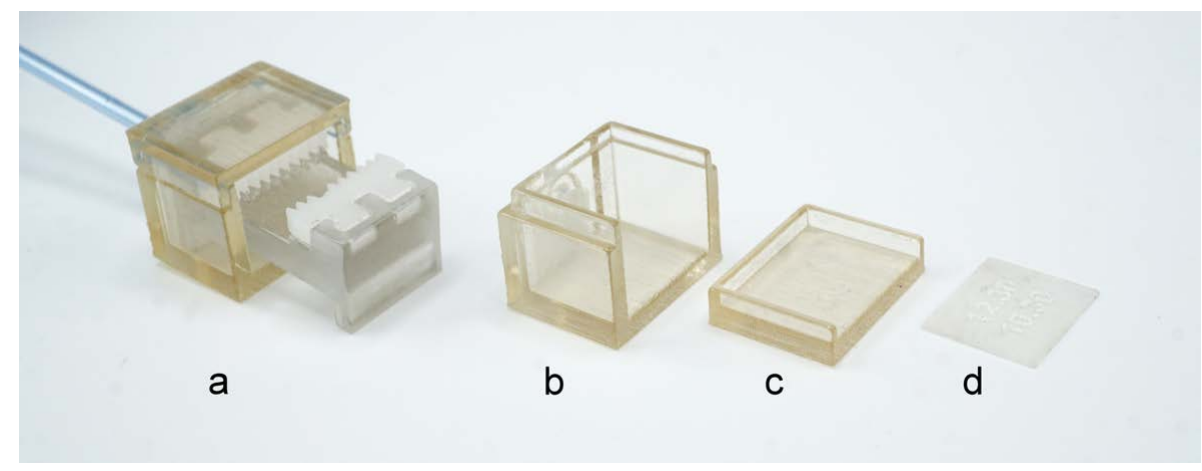

Figure 10.6: Realization of single-acting cylinder. a) Assembled cylinder with piston, b) housing, c) cap, d) seal.

\subsubsection{Manufacturization}

The presented pneumatic cylinders and pistons are printed on a Polyjet printer (Connex3 Objet260, Stratasys Ltd., Eden Prairie, MN, USA) in VeroClear material, standard quality, glossy finish. The glossy finish option implies that the top surfaces and side walls of the part are not covered with support material, which is important as supported faces are relatively rough and would cause excessive friction.

As shown in Figure 10.5 (left), the cylinder is printed in two pieces: the housing and the lid. Figure $10.6 \mathrm{~b}$, c shows these parts in the preferred print orientation which ensures that all four cylinder walls are printed with a glossy finish. Furthermore it is advisable to orient the parts such that the print head moves in the same direction (along $\mathrm{X}$-as of printer) as the piston would do in the cylinder, in order to obtain the smoothest possible finish for the cylinder walls along the direction of movement.

The next step is to join the parts together. Screws are no good option in small-scale MR safe applications due to space constraints, so bonding by glue (e.g. cyanoacrylate) is used. The challenge is to create an airtight bond without excess glue entering the cylinder cavity. It is therefore important to apply the right amount of glue and also to operate the cylinder at very low pressure right after assembly. In closed cylinder designs it is also recommended to cover the piston and seal in petroleum jelly (Vaseline) before gluing the housing together. Besides serving as lubricant, it also allows the seal to wipe away excess glue inside the housing before the glue has hardened out. A light patch of blue silicone (Loctite 5926) on the cylinder edge may be useful to improve airtightness and reduce risk of jamming.

Polyurethane tubes can be glued to the housing using cyanoacrylate (Loctite 406). An activator such as Loctite 770 should be applied to the polyurethane 
parts before gluing to ensure a good bond.

See Figure 10.6a for the resulting single-acting cylinder with a typical piston. The piston can be pushed in by hand and pushed out by pressurized air. The amount of leakage and friction can be qualitatively assessed by hand and quantitatively studied using appropriate equipment. Based on the crosssectional area, every $0.1 \mathrm{MPa}(\approx 1$ bar $)$ of pressure approximately results in an additional $12 \mathrm{~N}(\approx 1.2 \mathrm{~kg})$ of force.

\subsubsection{Double-acting cylinder}

A single-acting cylinder can push a piston in one direction only. In most applications a return stroke mechanism is required. In our applications a second single-acting cylinder is most suited for this. Alternatives are a mechanical (or permanent pneumatic) spring which requires fewer pneumatic tubes, but this results in much smaller net output forces for a given cross-sectional area.

The combination of two single-acting cylinders opposite to each other results in a double-acting cylinder. The cross-sectional area of the two opposite bores can be the same or different depending on the specific application. The piston is a single rigid object with two piston heads and no protruding rod. The specific piston shape defines the way it interacts with the environment, such as engaging with a toothed rack or firing a biopsy gun.

\subsection{Stepper motors}
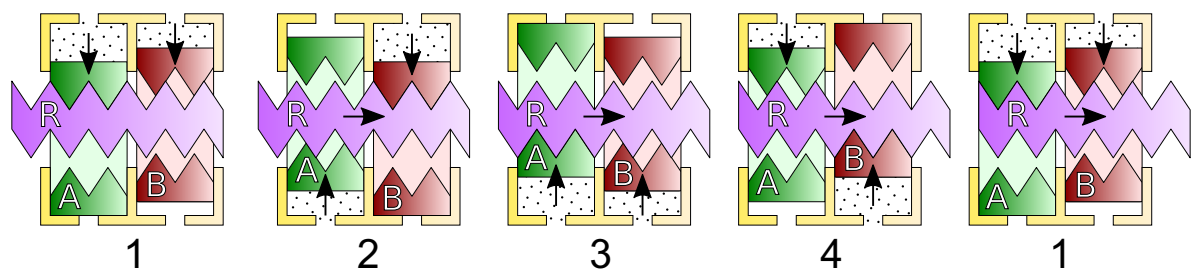

Figure 10.7: 1-4: Sequence of states in a two-cylinder pneumatic stepper motor, with rack moving from left to right. The rightmost state is identical to the leftmost one, but with the rack displaced to the right by one tooth pitch.

A pneumatic stepper motor can be constructed from two or three doubleacting cylinders that act on a toothed rack (or gear). In the two-cylinder version (Figure 10.7) the rack and pistons have teeth on two sides, while in the three-cylinder version (Figure 10.8) only one side is toothed.

The two-cylinder stepper motor design has four distinct states, shown in Figure 10.7. In each state both cylinders act on the rack, but only one of them can be fully engaged on it which is always the piston that moved formerly. 


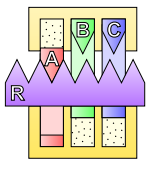

1

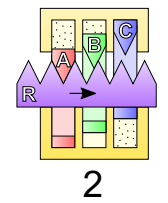

2

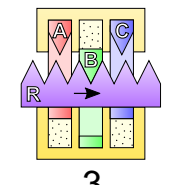

3

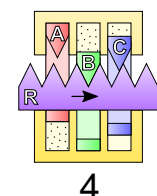

4

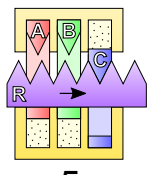

5

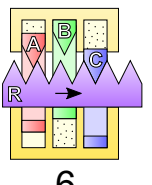

6

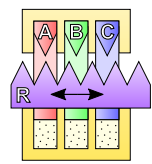

Free

Figure 10.8: 1-6: Sequence of states in a three-cylinder pneumatic stepper motor, with rack moving from left to right. The "Free" state (right) is the free-running state in which all pistons are moved up.

The consequence is that there is no backlash, but a hysteresis is present: when reversing direction, the observed rack position includes a certain offset compared to those in forward direction. While this offset could be measured and accounted for, a simpler approach is to approach each setpoint in a consistent (e.g. forward) direction.

The three-cylinder stepper motor design has eight distinct states, of which seven are shown in Figure 10.8. (The eight one, with all pistons engaging on the rack, is of no practical value.) In state 1, 3 and 5 exactly one piston engages on the rack and backlash may be present due to the finite clearance between piston and cylinder walls. In state 2, 4 and 6 the backlash is eliminated at the cost of the introduction of hysteresis like in the two-cylinder stepper motor design. Unlike in the two-cylinder design there is a free-running state, which allows to move the rack by external forces with negligible resistance.

\section{Design of two-cylinder stepper motor}

Like pneumatic cylinders, stepper motors come in various sizes. One specific design of a two-cylinder stepper motor is presented as an example and the associated files available for download [45]. Figure 10.9 shows a rendering of this stepper motor, which has dimensions $32 \times 30 \times 16 \mathrm{~mm}$ (excluding rack). It consists a housing with two top cover plates, two pistons, a rack and four seals. The cylinder cross-sectional area is $12 \times 10 \mathrm{~mm}$ and the teeth pitch of this particular design is $1.2 \mathrm{~mm}$, resulting in a step size of $0.3 \mathrm{~mm}$. The separation between the cylinders needs to be such that the cylinder pattern spacing is an odd multiple of the step size, as visualized in Figure 10.7. In this case it is $47 \cdot 0.3 \mathrm{~mm}=14.1 \mathrm{~mm}$, resulting in a central wall thickness of $14.1-12 \mathrm{~mm}=2.1 \mathrm{~mm}$.

In this stepper motor design the piston teeth are constructed by lasercutting a $2 \mathrm{~mm}$ acetal plate. The advantages of laser-cut teeth are sharper tips, increased strength, lower friction and reduced wear. These parts are inserted in the appropriate slots of the pistons and interact smoothly with the 3D printed teeth of the rack.

Figure 10.10 shows a realization of the stepper motor. The production 


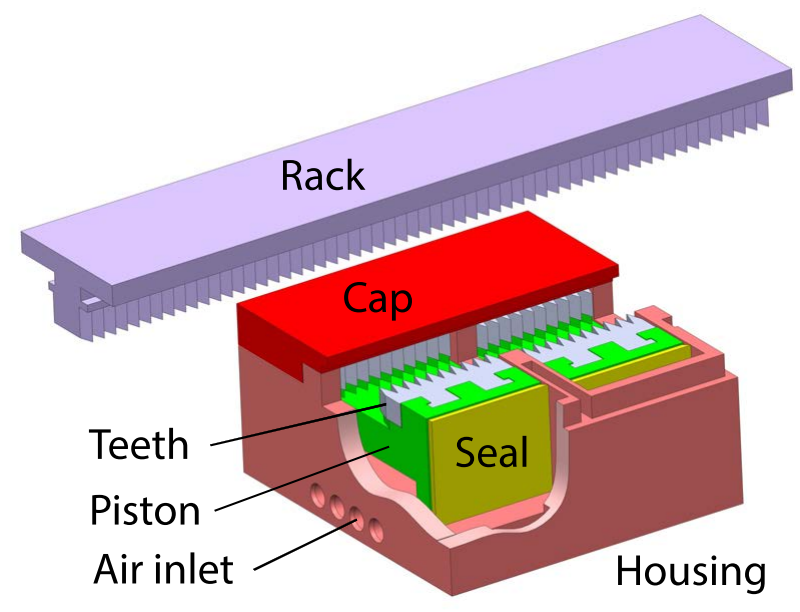

Figure 10.9: Cutout view of small-step two-cylinder linear stepper motor.

and assembly process is similar to that of the single-acting cylinder. Again, the parts are printed in VeroClear, glossy finish, cylinder walls facing up and aligned with the $\mathrm{X}$-axis of the polyjet printer. The air vents are initially filled with support material, but easily cleared with a small wire and the use of an air gun. Silicone grease (vaseline or equivalent) is used as lubricant, blue silicone acts as sealant and cyanoacrylate is used to glue the two caps to the housing. After assembly the cylinders are briefly operated at low pressure (without rack) to clear the cylinder walls from excess blue silicone and/or glue before hardening out while holding the housing together using clamps. After the glue has hardened out, the rack can be carefully inserted while slowly operating the cylinders at low pressure; some grinding and/or lubrication may be required to allow smooth movement of the rack. Finally the pressure can be increased to 0.1-0.4 MPa or higher.

\section{Curved stepper motor}

The rack of a stepper motor does not need to be linear: it can also follow a circular arc with some (finite) radius $R$. In fact, a linear stepper motor is a special case having $R=\infty$.

Figure 10.11 shows the design of a two-cylinder curved stepper motor. The two cylinders are not parallel, but angled in order to keep the piston movemement perpendicular to the curvature of the rack. Apart from this, the design principles are the same to those of the linear stepper motors.

The piston teeth should be shaped such that these interact with the rack 


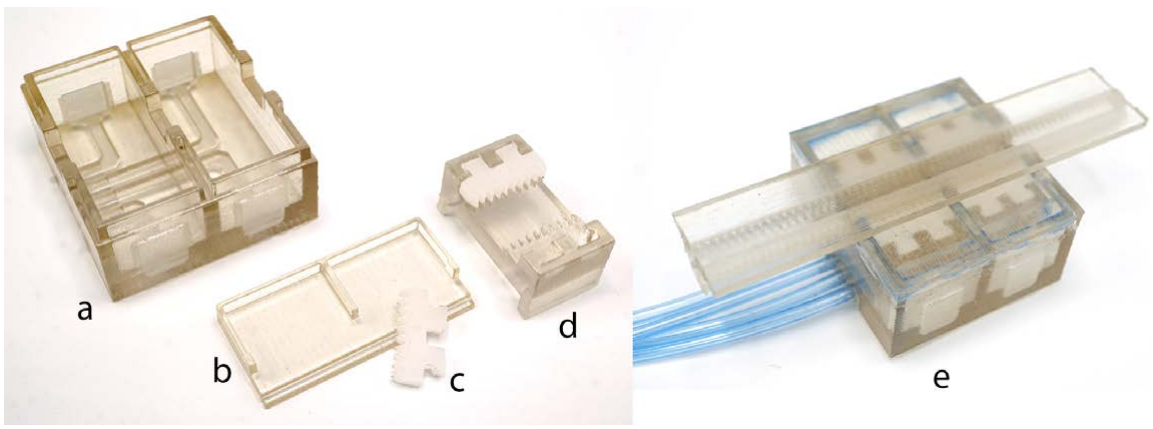

Figure 10.10: Realization of small-step two-cylinder linear stepper motor. a) housing, b) one cap, c) laser-cut teeth piece, d) piston with one teeth piece inserted, e) assembled motor.

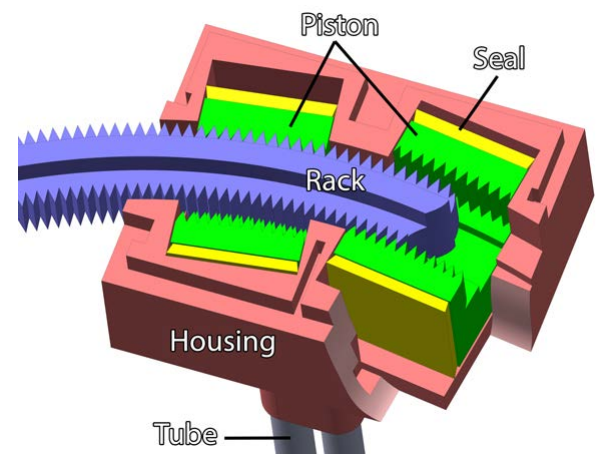

Figure 10.11: Cutout view of a curved stepper motor, exposing the two pistons, four seals and rack in the housing.

teeth at the same moment and minimize the risk of jamming. Figure 10.12 shows details of the teeth shapes in two positions of the piston and rack. The rack teeth are straight and symmetrical, while the piston teeth are slightly curved for two reasons. The first reason is made clear in subfigure a), in which it can be observed that the optimized piston teeth tips all make contact with the rack at the same moment so that load forces are as evenly distributed over all teeth as possible. The second reason is apparent in subfigure b). With the piston fully engaged on the rack, the non-engaging teeth tips must be opposite to the rack teeth (shown in dotted lines). This ensures that all piston teeth engage with the rack in a consistent manner, i.e. all pushing the rack to the left or all to the right. A non-optimized piston teeth shape (dashed lines) would have the possibility of jamming, making the motor less reliable. 

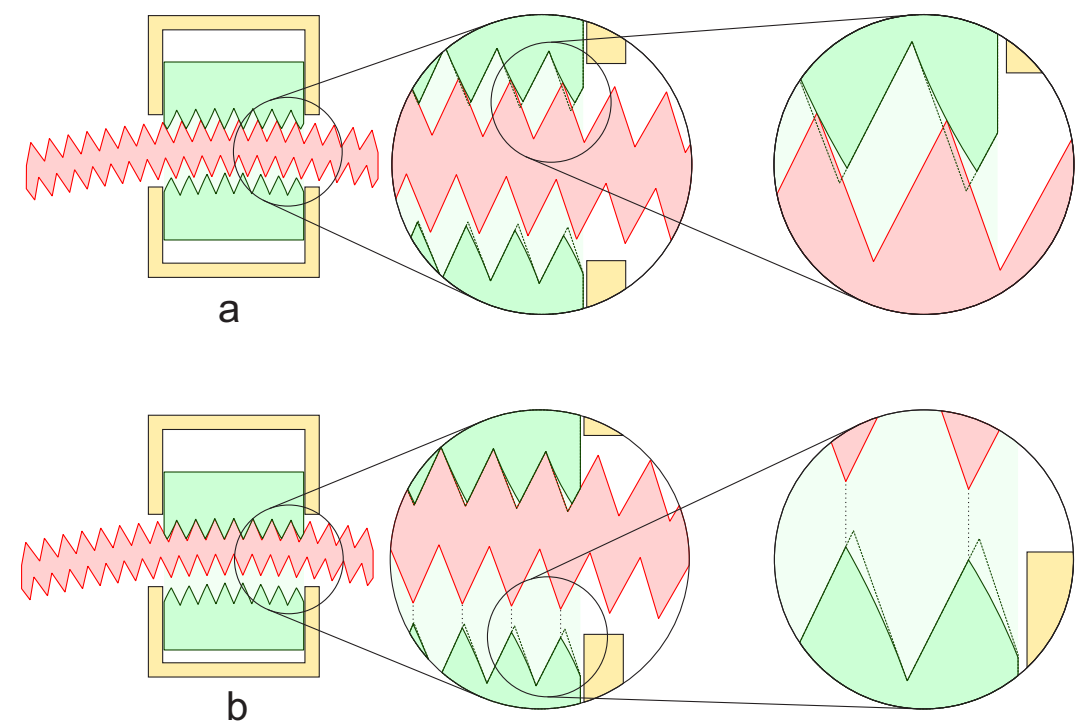

Figure 10.12: Analysis of teeth shape in curved stepper motor. The piston teeth have a special shape in order to optimize contact and reduce risk of jamming.

\section{Dual-speed stepper motor}

In an MRI environment the stepping frequency is limited to approximately $10 \mathrm{~Hz}$ when full force is needed. This would result in long procedure times when large distances are to be covered with high accuracy. A straightforward solution is to combine two (or more) stepper motors together on the same axis, allowing for both high-speed and high-accuracy movements.

A dual-speed stepper motor is essentialy a combination of two singular stepper motors with different step sizes arranged in a serial kinematic chain. In order to make efficient use of the space, each dual-speed motor consists of a single housing in which cylinders for all pistons of both singular stepper motors arranged in line. The two racks are positioned at opposite sides of the housing. This way the cross-sectional area of the dual-speed motor is the same as in the single-speed motor, while the extra length in the housing only occupies space in the direction of movement.

The key design aspect is the pitch sizes of both singular motors. The most straightforward approach is to combine a large-step motor for fast movements with a small-step motor for high accuracy. An alternative approach is to use two large-step motors with slightly different step sizes, exploiting the step size difference to making small steps.

Figure 10.13 shows the design of a generic dual-speed motor with size $50 \mathrm{x}$ $32 \times 14 \mathrm{~mm}$ (excluding racks). It consists of four pistons: the outermost two 


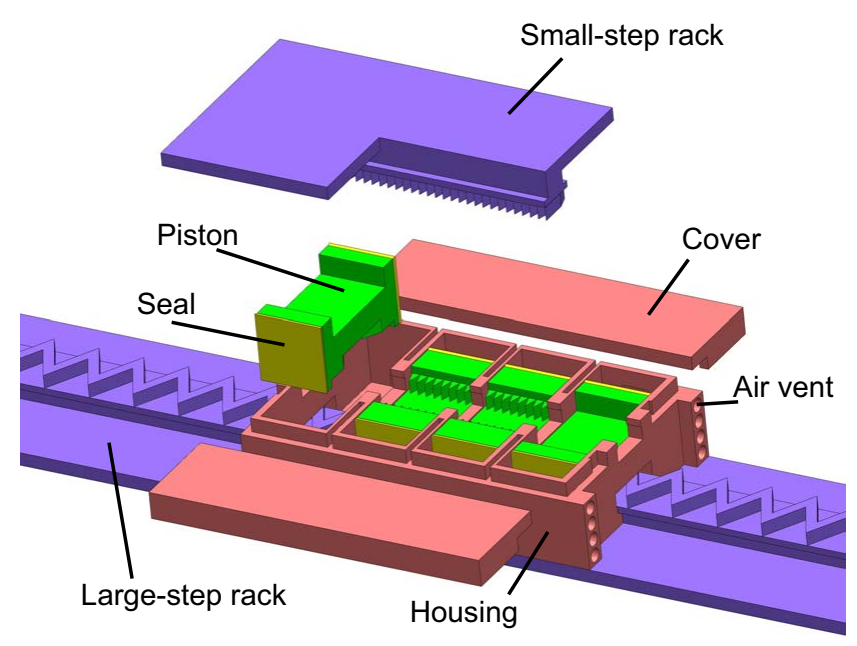

Figure 10.13: Dual-speed stepper motor design. The top rack has pitch $0.3 \mathrm{~mm}$, the bottom rack has pitch $1.7 \mathrm{~mm}$.

pistons operate the large-step rack with step size $1.7 \mathrm{~mm}$, while the innermost pistons operate the small-step rack with step size $0.3 \mathrm{~mm}$. Measurements have shown that the maximum force under load is $24 \mathrm{~N}$ at a pressure of $0.3 \mathrm{MPa}$, and positioning accuracy is $0.1 \mathrm{~mm}[52]$.

\subsection{Design of Sunram 5}

This section describes the kinematics and mechanical design of Sunram 5. It is driven by linear and curved stepper motors, dual-speed motor combinations and single-acting and double-acting pneumatic cylinders described earlier in this chapter. The actuators are specifically adapted to the needs of the respective axes. First the kinematic configuration is described, followed by details of the mechanical implementation.

\subsubsection{Kinematic configuration}

Figure 10.14 shows the kinematic configuration of Sunram 5. Figure 10.15 shows a photo of the Sunram 5 indicating the movement directions of the joints and cylinders.

Joint J1 is a curved stepper motor with a radius of $260 \mathrm{~mm}$. The teeth pitch is $1.5^{\circ}$, equivalent to a teeth distance of $6.81 \mathrm{~mm}$ and a step size of $1.7 \mathrm{~mm}$ along the curved rack. The total range is $35^{\circ}$ (93 steps). It is used for coarse 
Ch 10: Sunram 5: An MR safe robotic system for breast biopsy, driven by 194 pneumatic stepper motors

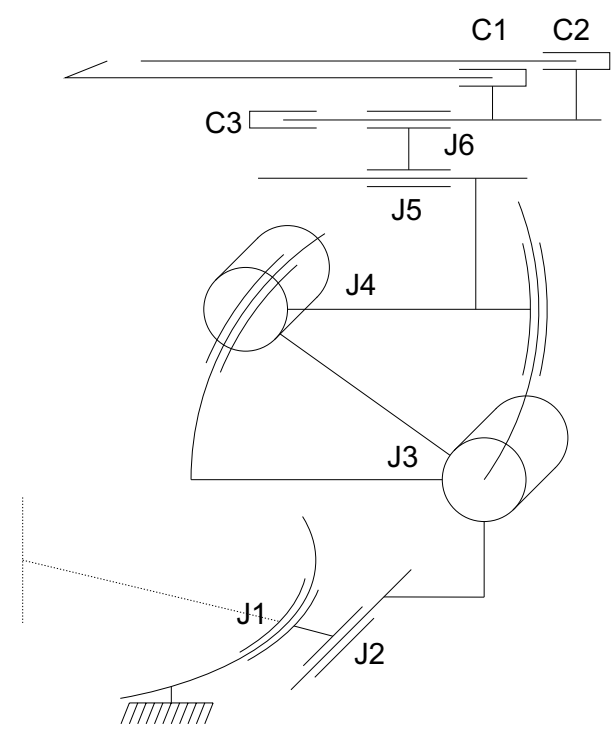

Figure 10.14: Kinematic configuration of Sunram 5 with joints $J 1-J 6$, biopsy gun cylinders $C 1$ and $C 2$, and emergency ejection cylinder $C 3$.

positioning of the robotic system and the curvature allows for a more favourable insertion angle near the borders of the workspace compared to pure linear motions. Joint J2 is a linear stepper motor with step size $0.3 \mathrm{~mm}$ and a range of $45 \mathrm{~mm}$ (150 steps). Joint J2 is used for fine lateral adjustments, but can also be used in conjunction with $\mathrm{J} 1$ to tilt the needle sideways over small angles to circumvent the grating of the breast fixation system and/or optimize the needle trajectory. Although joint J1 and J2 operate on different axes, the combination has characteristics of a dual-speed stepper motor because of the capability of both quick and precise lateral positioning over the full width of the workspace.

Joint J3 and J4 are rotational stepper motors that lift and tilt the needle holder vertically. Both joint J3 and J4 are single-speed curved motors with a radius of $62 \mathrm{~mm}$ and teeth pitch of $1.2^{\circ}$ corresponding to a step size of $0.3^{\circ}$ $(0.32 \mathrm{~mm}$ at $62 \mathrm{~mm})$ and a range of $40^{\circ}$ (133 steps).

Joint J5 is a linear stepper motor which moves the needle holder assembly forwards and backwards in small steps. The pitch size is $1.2 \mathrm{~mm}$ corresponding with a step size of $0.3 \mathrm{~mm}$ and the range of motion is $50 \mathrm{~mm}$ (167 steps). Joint $\mathrm{J} 6$ is a three-cylinder linear stepper motor with pitch size $5.1 \mathrm{~mm}$, step size $1.7 \mathrm{~mm}$ and range of motion of $61 \mathrm{~mm}$ (48 steps) along the same axis as $\mathrm{J} 5$, so joints J5 and J6 together form a true dual-speed stepper motor.

Cylinder $\mathrm{C} 1$ drives the inner needle of the biopsy gun forward over a distance of $19 \mathrm{~mm}$ and cylinder $\mathrm{C} 2$ slides the needle shaft over the inner needle over the 


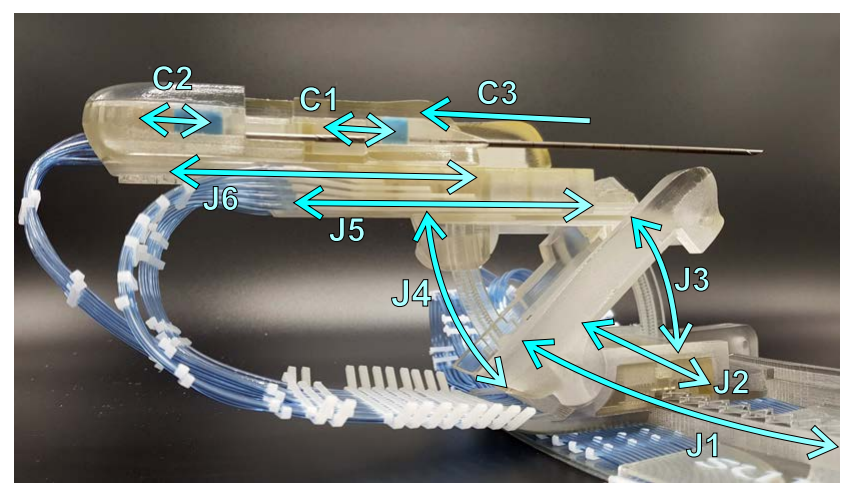

Figure 10.15: Photo of Sunram 5 with movement directions of joints $J 1-J 6$ and cylinders $C 1-C 3$.

same distance. Cylinder is C3 is the emergency needle ejection cylinder and it is effective when joint $\mathrm{J} 6$ is in free-running state.

\subsubsection{Mechanical design of Sunram 5}
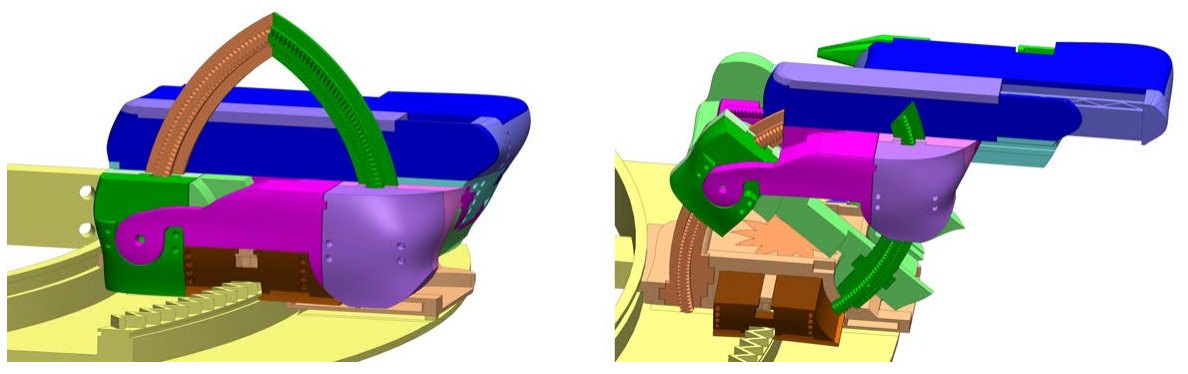

Figure 10.16: CAD drawing of Sunram 5, in compact (left) and extended (right) configurations.

Figure 10.16 shows two 3D CAD drawings of the Sunram 5, in compact and extended configurations. The robot consists of sixteen pneumatic cylinders in total, distributed over six singular stepper motors, two double-acting cylinders and one single-acting cylinder. The height of each cylinder is $10 \mathrm{~mm}$ and the nominal wall thickness is $2 \mathrm{~mm}$. With the exception of joints $J 3$ and $J 4$, all cylinders are oriented horizontally and distributed across three levels of approximately $14 \mathrm{~mm}$ each. This results in a total height of only $47 \mathrm{~mm}$ for the moving part of the Sunram 5 robot (excluding racks and cableguide).

Figure 10.17 shows the actuators of joint $J 1$ and $J 2$. The motor design 


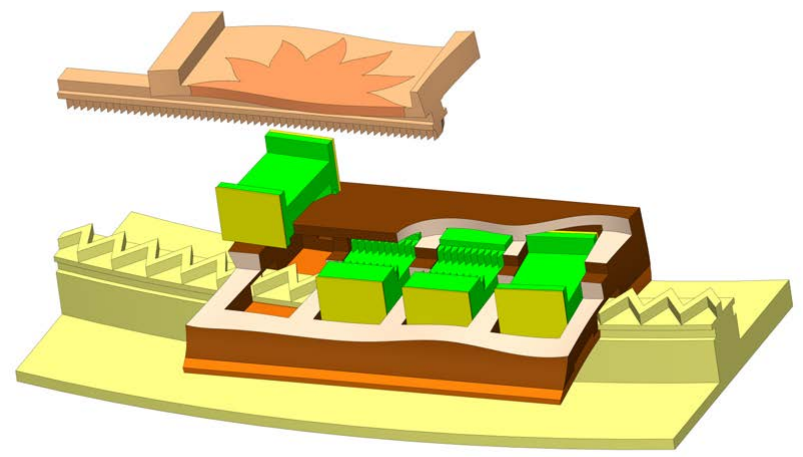

Figure 10.17: Actuators of joints $J 1$ and $J 2$ to drive the Sunram 5 sideways along the track. The curved bottom rack has step size $1.7 \mathrm{~mm}$ along the rack, the top rack has step size $0.3 \mathrm{~mm}$.

and cylinder arrangement are similar to that of the dual-speed stepper motor shown in Figure 10.13. The only significant difference is that joint $J 1$ is slightly curved with curvature radius $260 \mathrm{~mm}$, resulting in the outermost two cylinders being angled respective to the other cylinders. The small-step rack (joint J2) is connected to the base with a guiderail as shown in Figure 10.16, in order to reduce parasitic rotational movements in both $J 1$ and $J 2$.

Figure 10.18 shows a cross-section of joints $J 3$ and $J 4$ that lift and tilt the Sunram 5 vertically. Both joints are curved stepper motors similar to the one shown in Figure 10.11. The curved rack has a radius of curvature of $62 \mathrm{~mm}$ and the optimized teeth shapes of Figure 10.12 are used in the pistons which are laser-cut from $2 \mathrm{~mm}$ acetal and attached to the 3 -D printed piston with small pins.

Acrylic pins with diameter $3 \mathrm{~mm}$ are used as passive hinges in the axis of rotation of joints $J 3$ and $J 4$. These pins are partially visible in Figure 10.15 and these greatly reduce the amount of parasitic movements in the kinematic chain. Moreover, the hinge of either joint coincides with the curved rack of the other joint, resulting in a truss-like mechanical structure in extended configuration.

The needle insertion mechanism consists of one dual-speed stepper motor, one single-acting emergency ejection cylinder and two dual-acting biopsy gun cylinders.

The dual-speed motor is shown in Figure 10.19. It consists of a threecylinder large-step part with pitch size $5.1 \mathrm{~mm}$ and step size $1.7 \mathrm{~mm}$, and a two-cylinder small-step part with step size $0.3 \mathrm{~mm}$. The specific arrangement 


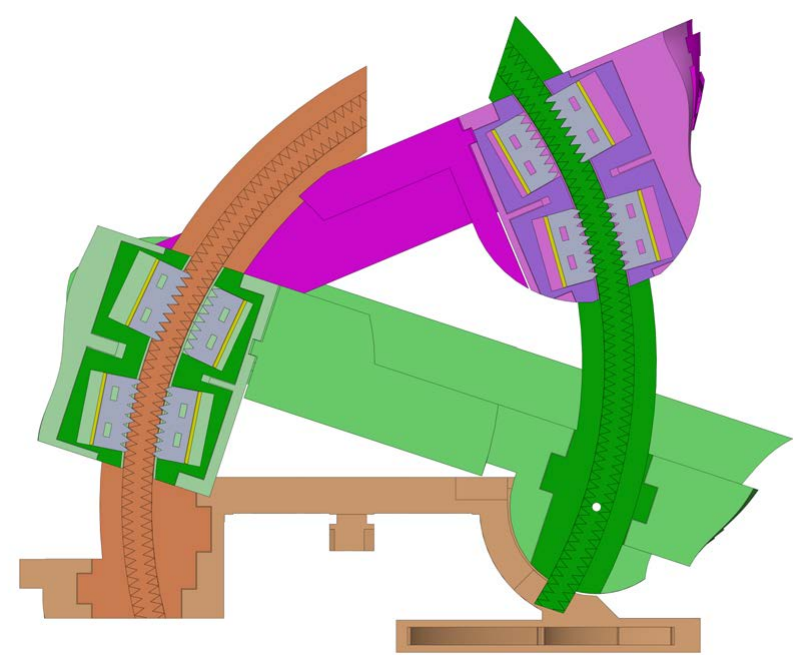

Figure 10.18: Cross-sectional picture of joints $J 3$ and $J 4$ which lifts and tilts the Sunram 5 vertically.

of the five cylinders allows for a telescopic expansion as shown in Figure 10.15. This expansion is needed to allow sideways movements of the Sunram 5 with the $100 \mathrm{~mm}$ biopsy needle installed, without colliding with the frame of the breast fixation system.

Figure 10.20 shows the emergency needle ejection and biopsy gun mechanisms. A single-acting cylinder with dimensions $13 \times 10 \mathrm{~mm}$ is used for the ejection mechanism and it is connected to the needle motor's large-step rack as shown in Figure 10.21. The ejection mechanism can be activated whenever the three pistons of the large-step needle stepper motor (Figure 10.19) are all retracted ('Free' state in Figure 10.8).

The biopsy gun consists of two double-acting cylinders. Its respective pistons have a smaller and a larger piston head and this asymmetry allows to attach needle sockets to the side of each piston in a compact design. The stroke of both cylinders is $19 \mathrm{~mm}$ and the total length of biopsy gun is approximately $100 \mathrm{~mm}$.

\subsection{Control of pneumatic devices}

Each pneumatic cylinder is controlled individually by one 5/2-way valve of type Festo MHA2-MS1H-5/2-2 (Festo AG and Co. KG, Esslingen, Germany), located in the valve manifold of the controller located outside the Faraday cage of the MRI scanner. The valve is a high-speed solenoid valve with nom- 


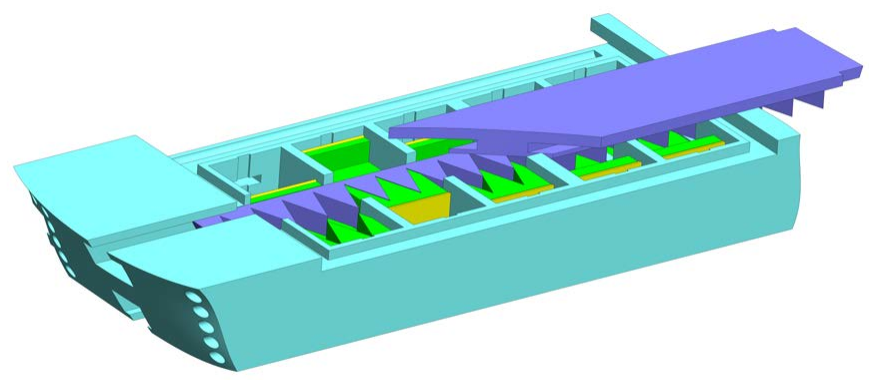

Figure 10.19: Cut-out view of Sunram 5's dual-speed needle insertion motor. The three cylinders in front operate the large-step rack on top with step size $1.7 \mathrm{~mm}$.

inal airflow $100 \mathrm{~L} / \mathrm{min}$ and a specified switching time of $1.9 \mathrm{~ms}$. The use of more conventional internally-piloted valves such as PV5211-24VDC-1/8 (TechniComponents B.V., Waalwijk, The Netherlands) is also possible. In an MRI environment the tube length should be around $5 \mathrm{~m}$ and its outer diameter can be 3 to $4 \mathrm{~mm}$. In the Sunram 5 the last $0.5 \mathrm{~m}$ of the tube has a diameter of $2 \mathrm{~mm}$ in order to provide sufficient flexibility in the various degrees of freedom. The cable bundle is managed such that it exits the robot at a fixed point in the frame.

It is not recommended to use $2 \mathrm{~mm}$ tubes for the full distance between controller and robot as this would constrain the airflow too much, resulting in reduced output force at the same stepping frequency. Likewise, it is not recommended to use $6 \mathrm{~mm}$ tubes to connect the robot to the controller as the associated cable bundle would be quite big and the large volume of air inside the tubes would require the use of bigger valves and additional air supply capacity in order to provide sufficient airflow.

Sunram 5 contains sixteen pneumatic cylinders with a total of 31 pneumatic connections (one cylinder is single-acting). It is theoretically possible to lower this number of tubes in different ways. Cylinders belonging to different stepper motors could share the same tube pairs, at the cost of increased controller software complexity and hysteresis effects. It is also possible to control a cylinder with a single pneumatic tube if full force is not needed. In that case a constant return spring action must be present with (approximately) half the force of the active stroke. This could be provided by e.g. a constant pressurization of the other end at half the system pressure, or equivalently by pressurization of a smaller cylinder area at system pressure.

In Sunram 5 the pneumatic manifold is controlled by an Arduino microcon- 


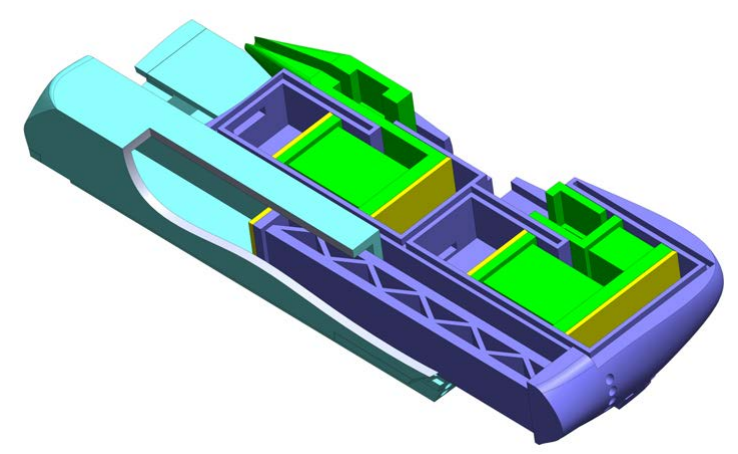

Figure 10.20: Cut-out view of Sunram 5's emergency eject single-acting cylinder (left) and the two dual-acting pneumatic cylinders for the biopsy gun (right).

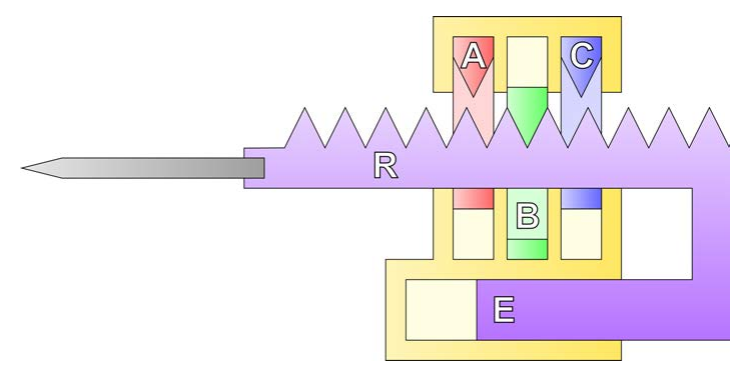

Figure 10.21: Needle safety mechanism. Pistons A, B and C together with rack R form a three-cylinder stepper motor, while piston $\mathrm{E}$ (connected to rack $\mathrm{R}$ ) is part of a single-acting cylinder.

troller, which is in turn commanded by a user interface. Figure 10.23 shows a photo of the controller. Besides the valves and Arduino controller it also includes a pressure adjustment knob with gauge, internal pressure tanks, an emergency stop button, six sliders for direct control of the stepper motors, a display for status information accessible by a menu dial and a tri-state biopsy fire switch. On the right panel there are connections for electric power, air supply and USB.

Feed-forward is used as the control method. This is sufficient provided that the initial position is known and no steps are skipped thereafter. The calibration process involves operating the robot to a predetermined position by either visual guidance or by operating each axis towards its endstops at low pressure (or high speed). In order to guarantee that no steps are skipped after calibration, motor forces must exceed maximum joint load forces by a certain safety margin. A typical stepper motor in Sunram 5 is capable of exerting over $50 \mathrm{~N}$ at $0.5 \mathrm{MPa}$ pressure, which is more than enough to insert a sharp needle in 
Ch 10: Sunram 5: An MR safe robotic system for breast biopsy, driven by 200 pneumatic stepper motors

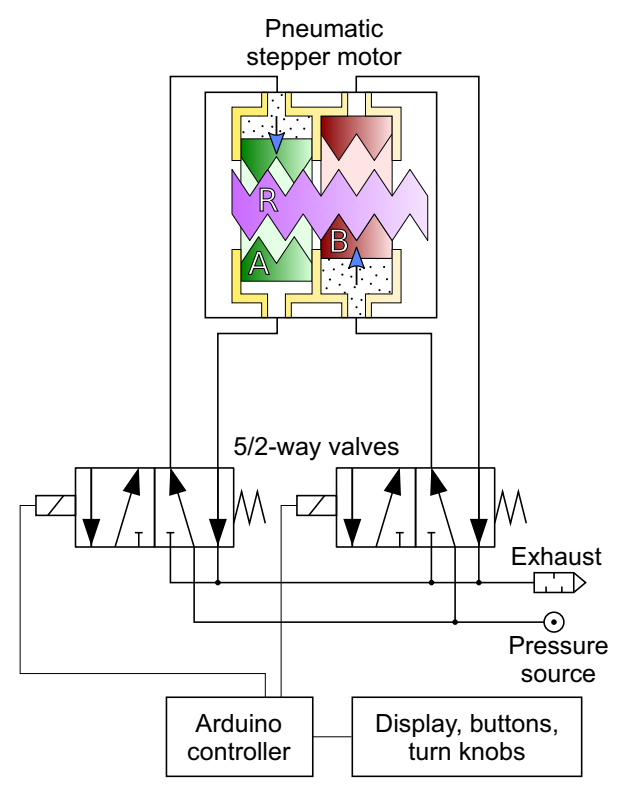

Figure 10.22: Schematic for controlling a two-cylinder stepper motor using two $5 / 2$-way valves.

breast tissue. It is also possible to increase the stepping frequency beyond the bandwidth when executing free-air movements in order to minimize the total procedure time.

While it is certainly possible to incorporate incremental position encoding in an MR safe robot using e.g. fiber optics, the associated complexity and additional space requirements are considerable. If additional space would be available, it could as well be used to increase the cylinder cross-sectional areas instead, leading to higher motor forces and reducing the necessity of positional encoders.

The presence of the robot inside the MRI scanner offers a potential indirect way of position feedback. By including suitable passive fiducials such as oil capsules in the robot design the relative location of the various linkages can be measured with sub-millimeter accuracy in a typical MRI scanner.

In the clinical workflow the breast is immobilized by a fixation system to which the robot is attached. Fiducials in the frame provide a way of defining the robot-MRI coordinate transformation, allowing to represent the target lesion in robot coordinates. After choosing the desired path trajectory by the radiologist, the joint configuration of Sunram 5 can be computed.

The specific serial kinematic design of Sunram 5 allows for straightforward computation of the joint configuration corresponding with the desired needle 


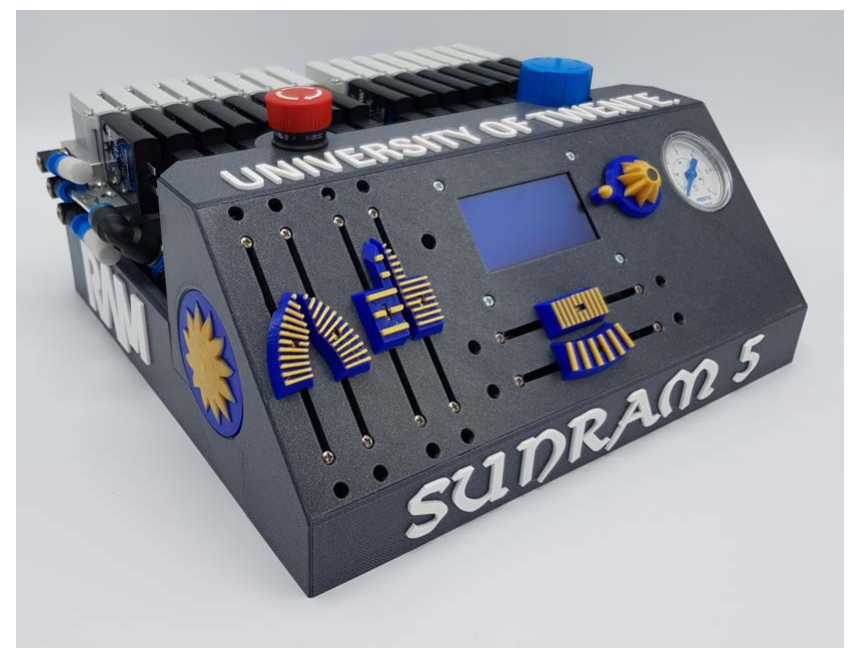

Figure 10.23: Sunram 5 controller with user interface.

insertion trajectory. The needle lift/tilt and insertion mechanisms all move the needle in the same vertical plane, so the first step is to align that vertical plane with the target lesion by choosing appropriate joint coordinate values for joints $\mathrm{J} 1$ and J2. The next step is to calculate the joint coordinate values for joints $J 3$ and $J 4$, aligning the needle holder with the lesion. Finally, joint coordinate values for joints $J 5$ and $J 6$ are calculated based on the distance from needle tip to lesion. The biopsy gun can now be fired and the needle with specimen extracted.

\subsection{Evaluation of stepper motors and Storm- ram 4}

Stepper motors can be characterized by finding the relation between output force, system pressure and stepping frequency. In a full robotic manipulator such as the Sunram 5, relevant characteristics are the needle tip positioning accuracy and precision and the average travel time in an MRI environment.

\subsubsection{Stepper motor force}

Sunram 5 consists of several cylinders and stepper motors. The theoretical output force of a pneumatic piston can be calculated from its cross-sectional area multiplied by the system pressure. The interaction of a piston with a straight or curved rack by means of the wedge mechanism results in a transfer 
Ch 10: Sunram 5: An MR safe robotic system for breast biopsy, driven by 202 pneumatic stepper motors

of force with a specific leverage, as determined from the teeth pitch and depth. The actual output force is lower due to friction in the sliding parts and can be measured.

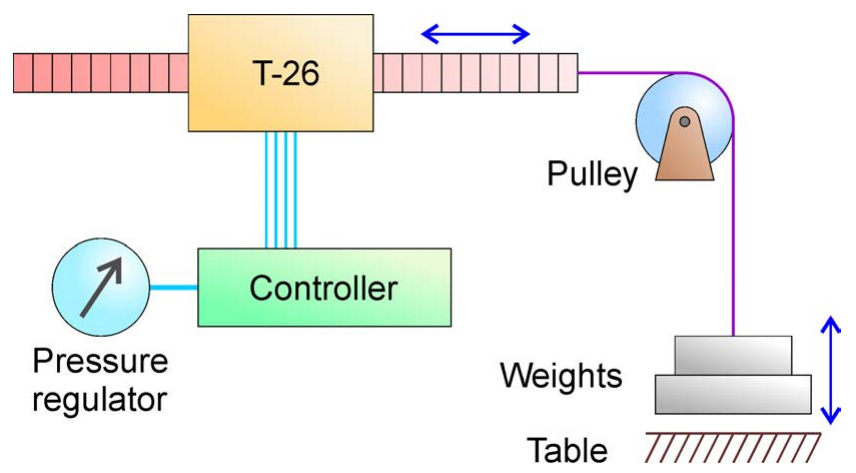

Figure 10.24: Schematic setup for stepper motor force measurements.

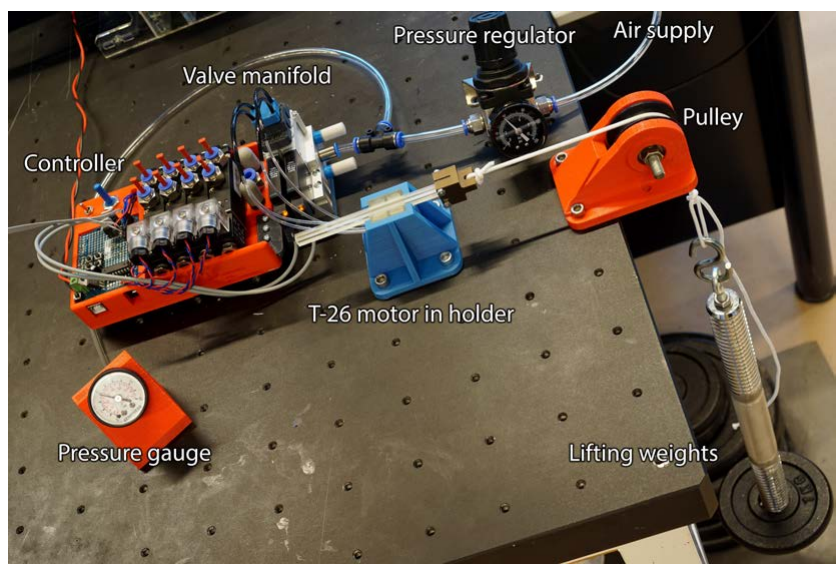

Figure 10.25: Photo of force measurement setup for T-26 stepper motor.

Figure 10.24 shows the schematic setup for evaluating the stepper motor force as function pressure and stepping frequency parameters. A set of masses with known weights are used to generate forces, transferred to the motor by means of a rope over a pulley. The pressure is adjusted to the lowest level that the motor can still lift the given masses without skipping steps.

Figure 10.25 shows a photo of an actual setup and Figure 10.26 shows the pressure-force graph for this specific motor. It can be observed that there is a good linear relationship between pressure and force, with a maximum of $62 \mathrm{~N}$ at a pressure of $0.65 \mathrm{MPa}$. Given the cylinder cross-sectional area of $10 \times 10 \mathrm{~mm}$ 


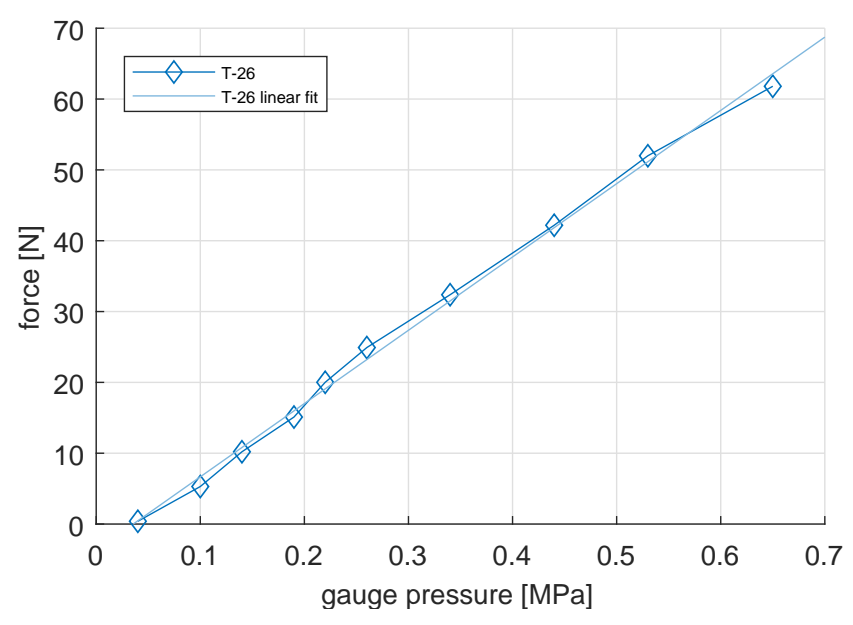

Figure 10.26: Pressure-force graph for the T-26 stepper motor.

and the leverage factor of 2.4, this results in a mechanical efficiency of $43 \%$ [50].

At a relatively low pressure of $0.15 \mathrm{MPa}$ the force of $11 \mathrm{~N}$ is already large enough to move a Sunram 5-like robot along all its axes, except for inserting the needle through relatively stiff tissue for which more pressure is needed.

Besides this motor, the authors of this chapter earlier analyzed and reported about ten different individual stepper motor implementations with mechanical efficiencies ranging from $22 \%$ to $76 \%$ [46, 47, 52]. Relatively low efficiencies $(22 \%-34 \%)$ were found for the Stormram 1 motors which have cylinder crosssectional dimensions of $20 \times 4.1 \mathrm{~mm}$. Its $5: 1$ aspect ratio results in a relatively high perimeter per surface area ratio and the low height of $4.1 \mathrm{~mm}$ makes effective sealing challenging [46]. This issue is solved by focusing on squareshaped cylinders with comparable width and height in all subsequent designs.

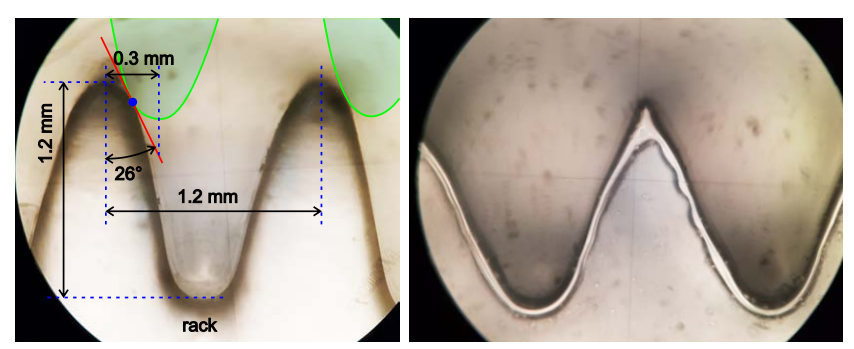

Figure 10.27: 3-D printed (left) and laser-cut (right) teeth with pitch size $1.2 \mathrm{~mm}$, as seen under a microscope. 
Ch 10: Sunram 5: An MR safe robotic system for breast biopsy, driven by 204 pneumatic stepper motors

Another case of low measured efficiency (24\%) was found in certain motors with 3 -D printed teeth with small pitch size (1.2 mm or smaller). Examination under a microscope reveals that the associated teeth tips are rounded, as shown in Figure 10.27 (left), resulting in a reduced leverage factor and increased risk of jamming[52]. This issue is solved by using laser-cut teeth in the pistons and/or the rack, which result in sharper tip shapes as shown in Figure 10.27 (right).

\subsubsection{Stepping frequency}

The maximum stepping frequency is another important characteristic and this is mainly dependent on the tube length (approximately $5 \mathrm{~m}$ in an MRI environment). Measurements have shown that the bandwidth is approximately $10 \mathrm{~Hz}$ [47]. One factor is the limited speed of pressurized air in the tubes which cannot exceed the speed of sound $(343 \mathrm{~m} / \mathrm{s})$ and is further restricted by the friction in the tubes. Another important factor is the finite volume of air inside the tubes (24.5 mL when using $4 \mathrm{~mm}$ tubes with $2.5 \mathrm{~mm}$ inner diameter), which implies that any further increase in tube thickness (to reduce wall friction) would require the use of bigger valves with larger orifices. Nevertheless, the stepper motors in Stormram 4 have shown to be able to operate at frequencies up to $65 \mathrm{~Hz}$ when moving in free air, as full force is not needed there.

\subsubsection{Accuracy}

Positional accuracy and overshoot have been studied for several stepper motors. Measurements have shown that hysteresis is present in two-cylinder stepper motors, with a magnitude of $60 \%-80 \%$ of the step size. The positional accuracy depends on the step size due to discretization: by design, the lower bound is half the step size in a singular stepper motor. In dual-speed stepper motors the accuracy can be as good as $0.1 \mathrm{~mm}$ [52]. The repeatability or precision is very good in general, measured to be as good as $0.01 \mathrm{~mm}$ [47].

\subsubsection{Stormram 4 evaluation}

Stormram 4 is the predecessor of Sunram 5. While it does not have the dualspeed motors that Sunram 5 has, the kinematic design is comparable and its measurement results give insights into the projected capabilities of Sunram 5 .

Figure 10.28 shows the experimental setup for evaluating the accuracy and precision of Stormram 4 in free air. The Stormram 4 was programmed to approach all 35 targets in succession by navigating through a sequence of predefined waypoints. Afterwards the offsets of each puncture from its respective target were measured. The average precision was found to be $0.71 \mathrm{~mm}$ in horizontal direction and $0.21 \mathrm{~mm}$ in vertical direction. Also, a bias of $1.0 \mathrm{~mm}$ was 


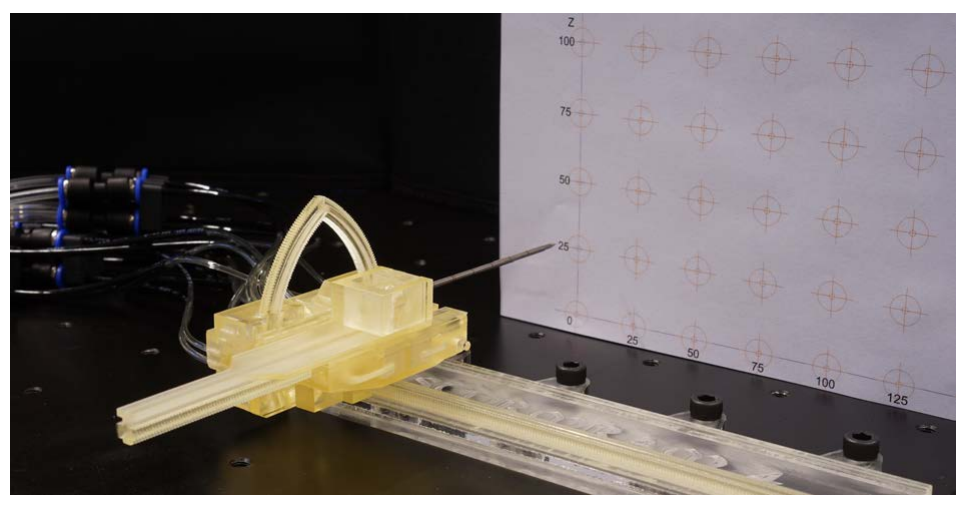

Figure 10.28: Stormram 4 accuracy experiment in free air.

found in horizontal direction due to manufacturing and/or calibration inaccuracies [50].

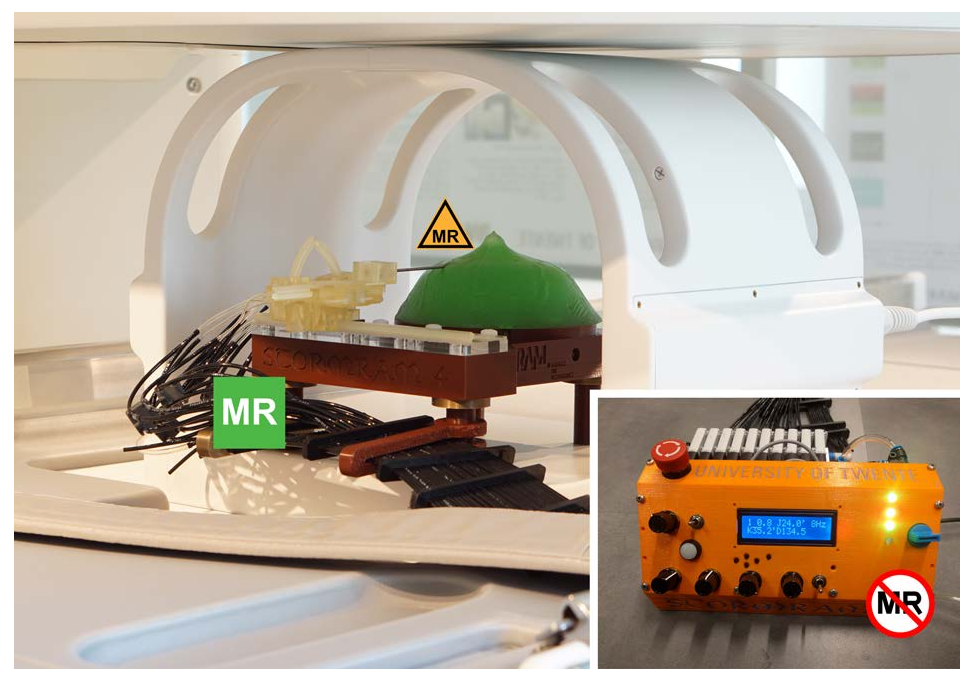

Figure 10.29: Measurement setup of Stormram 4 in a 0.25 T MRI scanner.

Figure 10.29 shows the experimental setup for accuracy measurements inside a 0.25 T (G-scan, Esaote SpA, Genoa, Italy) MRI scanner. The Stormram 4 was mounted on a table with ten fiducials in it. A PVC phantom was placed on the table, a pre-operative scan was made and a series of 30 targets inside the phantom were selected. For each target, first its MRI coordinates were transformed to the robot coordinate frame as defined by the positions of the fiducials, after which a suitable joint coordinate vector was calculated. The 
robot, which had been calibrated previously in its zero-position, was then operated through a user interface by rotating the turn knobs corresponding with each joint manually until the displayed joint coordinates matched the target coordinates. A confirmation scan was then taken and automatically segmented to reconstruct the needle tip location and measure the distance to the target coordinates. Tissue deformations were left out of scope in the experiment as no fixation system was used.

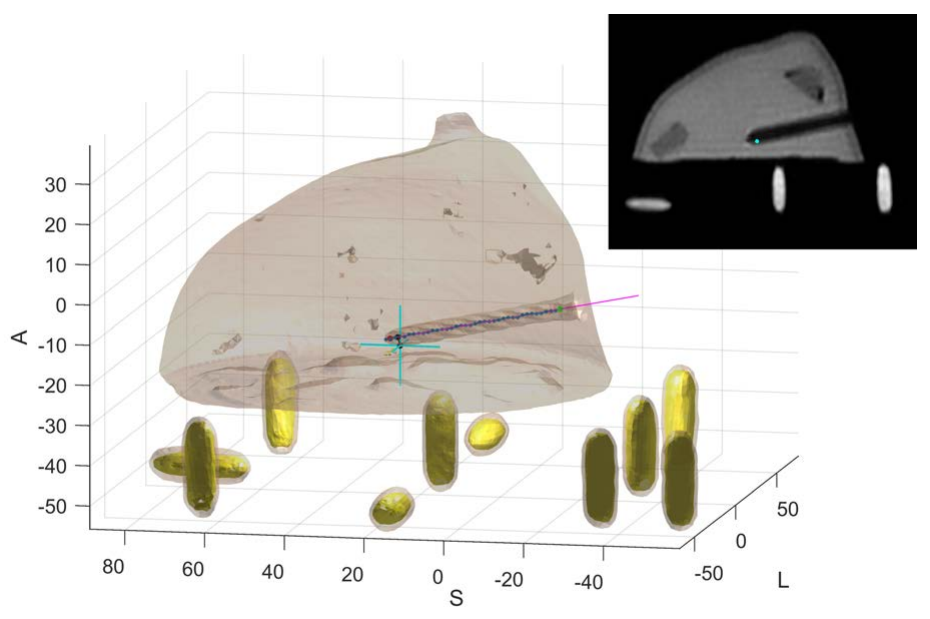

Figure 10.30: 3-D rendering of a MRI scan consisting of a phantom with needle inserted and ten fiducials. The crosshair indicates the target location.

Figure 10.30 shows an example confirmation scan after applying geometric distortion correction. The ten fiducials in the table define the robot coordinate system, as reconstructed automatically based on the orientations and interfiducial distances. The actual needle trajectory is also automatically reconstructed based on the connectivity graph of all low-intensity voxels in the scan, grouped into regions of equal shortest distance from a reference (low-intensity) voxel outside the phantom. The use of these automated algorithms allow to make optimal use of all available measurement data and also minimizes human estimation errors, resulting in sub-voxel precision of reconstruction parameters.

After targeting all 30 sites, the 3-D targeting error of Stormram 4 was measured to be $1.87 \pm 0.80 \mathrm{~mm}$ (range 0.69-3.57) [55]. 


\subsection{Conclusion}

Pneumatic devices are effective in actuating MR safe robotic systems. Several techniques for creating pneumatic actuators have been presented in this chapter: single-acting and double-acting cylinders, linear and curved stepper motors and dual-speed stepper motor combinations.

The presented actuators offer advantages over other state-of-art MRI actuators in terms of compactness, output force/torque, ease of control, rapid prototypeability and/or MR safety.

Several robotic systems have been developed for MRI-guided breast biopsy that are actuated by the described pneumatic cylinders and stepper motors. The latest generation, Sunram 5, has shown to be a compact and versatile prototype. Thanks to the dual-speed motor implementations on two axes it combines high speed with high accuracy, especially useful in an MRI environment in which long tubes are necessary which restrict the stepping frequencies.

The pneumatic cylinders and stepper motors are manufactured by $3-\mathrm{D}$ printing and laser-cutting. The single-acting cylinder model is useful in exploring sealing and manufacturization techniques, while the two-cylinder stepper motor design with laser-cut teeth combines high precision with low friction and good durability.

The amount of friction depends on many factors and varies from one motor implementation to another. Measurements on several different stepper motor implementations result in efficiencies from $50 \%$ to $76 \%$, provided that the teeth tips are sufficiently sharp, the cylinder cross-section is approximately square and the seal dimensions are optimized to minimize friction and leakage.

The kinematic and mechanical designs of Sunram 5 are extensively described in this chapter. Based on Stormram 4's measurement results, the projected needle tip accuracy in free air is less than one millimetre, while the expected phantom targeting accuracy in MRI is in the order of two millimetres.

Given the capabilities of Sunram 5 and earlier Stormram robots, the presented pneumatic stepper motor technology has shown to be a promising actuation technique for any MR safe robotic system. Concerning MRI-guided breast biopsy, Sunram 5 is not yet ready for clinical use. Additional research is needed before clinical trials can be conducted, especially on calibration, path planning and control, sterilization and safety. Sunram 5 has shown to be an advanced proof-of-concept which may shape the future of MRI-guided robotic interventions. 



\section{Part IV}

\section{MURAB / Tissue deformations}





\section{DESIGN OF AN END-EFFECTOR FOR ROBOTIC ASSISTED US GUIDED BREAST BIOPSY}

M.K. Welleweerd, F.J. Siepel, V.Groenhuis, J.Veltman, S.Stramigioli. Design of an end-effector for robotic assisted US guided breast biopsy. Submitted to the International Journal of Computer Assisted Radiology and Surgery (IJCARS), 2019 


\subsection{Abstract}

Breast cancer is the most common type of cancer among women worldwide. To confirm the diagnosis, a tissue sample should be acquired from the lesion for pathology. In the so called biopsy procedure, the sample is extracted from the lesion by a needle under image guidance from an ultrasound probe. The current process is sub-optimal due to manual insertion of the needle, inaccurate image feedback, and patient discomfort.

The aim of this paper is to present a US guided breast biopsy robotic end-effector to target breast tumours under real time image-feedback. Robots are more accurate, precise and stable than humans, and can therefore play an important role in increasing the efficiency and effectiveness of the current procedure. On top of that, a robotic arm carrying an ultrasound (US) probe can assist in $3 \mathrm{D}$ volume reconstruction and registration with other imaging modalities utilizing its known geometry and position sensors.

The presented end-effector supports the radiologist during the US guided biopsy procedure. In the envisioned procedure, the robotic arm is able to perform localization of the breast, 3D US volume acquisition and reconstruction, target identification and needle guidance. To enable the robot to execute these steps, the end-effector is equipped with a stereo camera setup, a picobeamer, US probe holder, a 3DOF needle guide and a needle stop.

Experiments were performed to determine the needle placement accuracy in the US plane. This was done by equipping the US probe with displaceable targets. The mean error w.r.t. the target in $y$-direction was found to be $0.03 \pm 0.36 \mathrm{~mm}$. The mean error in z-direction is $0.3 \pm 1.5 \mathrm{~mm}$. The needle stopping accuracy is $40 \mu \mathrm{m}$ with a maximum error of $1.18 \mathrm{~mm}$. The maximum holding force of the stop is around $6 \mathrm{~N}$. This is enough to stop the needle during a procedure.

The results are promising and are a step towards a more successful biopsy procedure which decreases the number of false positives in the outcome of the diagnosis and minimizes the patient's trauma.

\subsection{Introduction}

Breast cancer is the most commonly diagnosed cancer in women worldwide. In 2018 alone, nearly 2.1 million new cases were diagnosed [19]. For these women it is essential that the diagnosis is confirmed in an early stage of the disease.

An early detection is known to reduce mortality rates in breast cancer [106]. To identify lesions, several (imaging) methods can be used including self examination through palpation and imaging modalities such as mammography, ultrasound (US) scans and magnetic resonance imaging (MRI) scans. Mammography is most commonly used in clinical practice. If a lesion is detected, a 
tissue sample is needed to confirm malignancy. This tissue sample is acquired using a biopsy needle, after which the sample is sent to the pathologist.

Most often, the biopsy procedure is performed under US guidance. The radiologist navigates the needle based on US feedback. There are several disadvantages to this procedure. Extracting cells from the lesion may be difficult due to the small size of the lesion, or poor sensitivity due to difficulties in visualizing small tumors against a background of dense fibroglandular tissue [102]. Furthermore, needle insertion is hampered by tissue boundaries and the mass tends to be displaced by the exerted forces during needle insertion. The biopsy is repeated if the lesion is not hit at the previous attempt. The consequence of before mentioned issues is that practitioners should be experienced to be successful. However, clinicians who frequently use this technique often suffer from fatigue and work-related musculoskeletal discomfort [115]. These work-related issues will become more frequent because over the past years there has been an increase in breast biopsies due to wider access to population screenings for breast cancer.

Robotics can play an important role in the above mentioned challenges. Robots are more accurate, precise and stable than humans. On top of that, robots do not experience fatigue and the time per patient can be brought down using robots [88]. Furthermore, a robotically steered US probe can produce accurate 3D US volume reconstruction. The US probe position can be acquired with high precision utilizing the sensors in the robot and with coordinated movements uniformly spaced slices can be produced. The accuracy of a biopsy benefits of image fusion of preoperative images acquired by e.g. MRI with intra-operative data like US [99]. If the robot 'knows' its relative position to the breast and is able to generate a precise 3D US volume, this can ease registration. Because of these advantages, the chance of a false negative during a robotic assisted US guided biopsy is potentially lower than with a regular procedure and patient discomfort and cost can be brought down.

Thus, robotic assistance during US guided breast biopsies is beneficial by providing a stable hand and real time image feedback. Previous studies focused mainly on the design of support mechanisms to assist the radiologist and to perform minimal invasive procedures safer, cheaper and more precise. One key step in a robotic assisted biopsy, is determining the position of the target relative to the biopsy device. This can be performed using pre-operative imaging. In $[76,91]$, optical tracking is used to relate the pre-operative US images to the robot. Nelson et al. used a laser scanner to relate a pre-operative 3D US acquisition to the current position of the breast [95]. Using just pre-operative imaging is advantageous because the trajectory planning is not influenced or restricted by e.g. US probe position but lacks real time information to correct for deformation. Several studies utilized real time US guidance as well. The position of the US probe with respect to the needle can be tracked optically, 
calculated based on joint sensors of the robot(s) holding the probe and/or the needle, or measured if the position of the US probe is static with respect to the needle base frame $[68,77,84,91]$.

In addition, there are several approaches to needle insertion. Liang et al. presented a 6 DOF robot holding a 3D US probe with the needle fixed to the probe. Mallapragada et al. presented a needle which had a fixed insertion orientation with respect to the probe, but were able to manipulate the tissue $[89,90]$. Other studies presented setups in which the needle/needle guide has some degrees of freedom in the image plane of the US probe $[18,68,83,116]$. In some cases, the needle had degrees of freedom out of the US plane as well or the US probe had degrees of freedom also [2, 75, 131]. The advantage of being able to move the needle independently of the US probe is that there is more freedom in path planning of needle insertion. However, if the needle moves out of the US plane, the advantage of US feedback is partly diminished.

The above-mentioned studies show that the introduction of robotics to the biopsy procedure is advantageous for the accuracy of the procedure. However, to truly benefit from developments in the area of robotics such as the medical certified robotic arms, there is the need for an all in one solution. If one tool enables a robotic arm to autonomously perform all steps of the breast biopsy, the system becomes less complex and expensive, and inter-system calibration errors are ruled out. This will lead to a higher accuracy and faster acceptance in the medical world [7].

The aim of this paper is to present an end-effector which enables an offthe-shelf robotic arm to autonomously perform all possible steps of a robotic assisted biopsy: Localization of the breast, 3D US volume acquisition and reconstruction, target identification and needle insertion.

\subsection{Method}

\subsubsection{Design considerations}

In the envisioned robotically assisted US guided biopsy procedure the robot is able to perform several steps (Figure 11.1). During all these steps, the patient is positioned in prone position, the same configuration as during an MRI scan of the breast. This reduces motion artefacts and simplifies registration with a pre-operative MRI scan. It is assumed that before the robot starts working a breast MRI scan has already been taken. Multi-modality markers, visible in MRI, US and on camera were attached to the breast.

The first step the robot then takes is locating its position with respect to the breast. It does so by moving around the breast and detecting the markers with cameras. Doing so, the MRI data can be registered with the optical data. By using the markers' relative positions and projections of a projector, possible 
deformations with respect to the pre-operative MRI data can be accounted for.

In case of 3D US data acquisition, the robot scans the $2 \mathrm{D}$ linear probe over the breast surface. The volume is built up by streaming the $2 \mathrm{D}$ images with their corresponding position data to a reconstruction algorithm. To optimize the scanning area, it is necessary to navigate as close to the bed as possible. Therefore, the probe should be tilted with respect to the robot flange (see Figure $11.1 \mathrm{~B})$.

Unlike the common US guided biopsy, with the patient in suppine position, the robotically assisted biopsy will be performed with the patient in prone position. During the needle insertion, the needle tip should be within the Field Of View (FOV) of the US transducer. This allows for real time image feedback of both the needle tip and the tissue deformation due to needle insertion. The needle tip should be aligned with the lesion in the breast and as parallel as possible with the transducer for needle visibility. In practice, this leads to a needle insertion point positioned at approximately $3 \mathrm{~cm} . .5 \mathrm{~cm}$ from the edge of the transducer. Furthermore, the needle is preferably inserted parallel to the chest wall because this reduces the risk for pneumothorax. Due to the beforementioned restrictions, the anticipated pose of the probe during a biopsy is will be as shown in Figure 11.1 C.

The robot should position and orient the needle guide based on the radiologist's instructions. Therefore, the needle will be a 3DOF mechanism which is able to aim the needle at a target in the plane of the US probe (Figure 11.1 D). Essentially, there are four variables to solve for in needle placement: Three joints define the position and orientation of the needle guide, and there is the depth of the needle insertion. The needle orientation and depth is determined by the target and the insertion position. The remaining two joints determine the position of the needle guide and form a planar two-link manipulator [94]. The workspace of this two link manipulator is defined by the needle insertion rules and the diameter of the female breast, which is approximated to a maximum of $18 \mathrm{~cm}$ [69]. The aim for the needle guide is to successfully perform biopsies on lesions with a size ranging from $4 . .10 \mathrm{~mm}$. This range of lesion sizes is difficult to see on US images but can be recognized on MRI images.

\section{Needle stop}

During the insertion phase, the radiologist inserts the needle in the breast through the needle guide. To optimize the accuracy of this step, the needle guide should limit the movements of the needle to just the direction of insertion. In the current procedure, the correct insertion depth is based on markings on the needle and the US image feedback. The accuracy of the envisioned procedure is entirely independent of the user. Therefore, the needle guide should also be able to stop and hold the needle at the desired position. To stop the needle, the break should be able to exert a force higher than the 


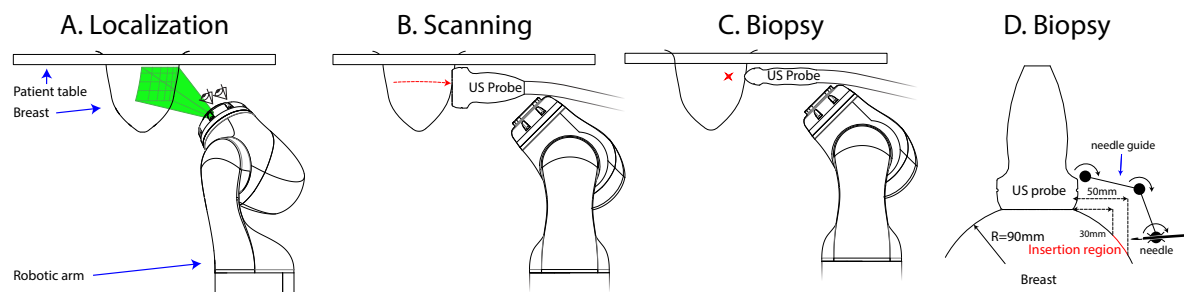

Figure 11.1: The workflow during a robotic assisted biopsy. A: The robot scans the breast with cameras and registers the breast surface by projecting light or recognizing markers. B: The robot scans the breast with a $2 \mathrm{D}$ US probe for $3 \mathrm{D}$ US volume reconstruction. C: The robot visualizes the target in the US image. D: The robot targets the lesion by aiming the needle guide to the correct location. In situations $\mathrm{B}$ and $\mathrm{C}$ a angle of $45^{\circ}$ of the probe w.r.t. the flange is beneficial to come as close to the chest wall/patient table as possible.

insertion forces. These forces may be expected to have a maximum around $3.5 \mathrm{~N}[1,134]$. Preferably, the mechanism functions independently of needle thickness and length and is designed such that it can be substituted or sterilized easily after usage.

\subsubsection{Materials}

Solidworks (Dassault Systèmes SE, France) was used to create the final design, as shown in Figure 11.2. The design is adapted for a KUKA MED 7 R800 (KUKA GmbH, Germany) and optimized for the situations described in the previous section.

From the scanning and biopsy phase it was clear that the robot should be able to move as close as possible to the patient table. This is achieved with a US probe placement which is rotated with respect the flange connection. This is where the end-effector is attached to the robotic arm. The probe is connected
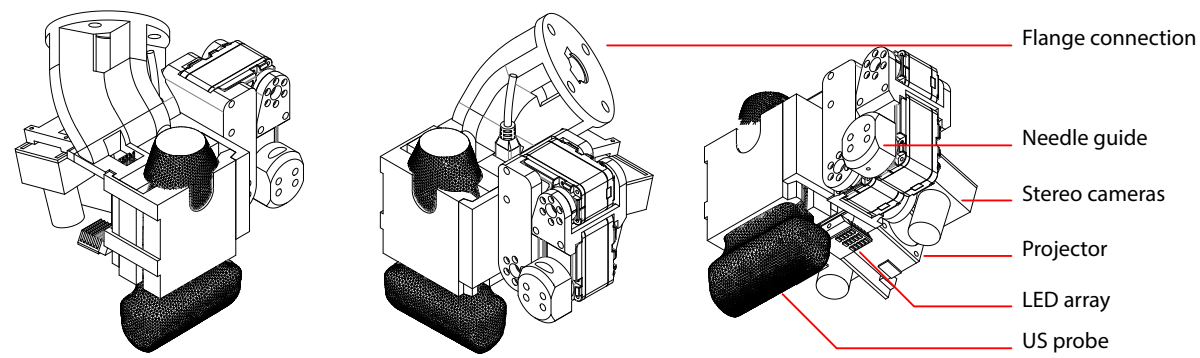

Figure 11.2: Isometric projections of the end-effector design. The US probe tip is rotated $45^{\circ}$ w.r.t. the robot flange around both $\mathrm{x}$ and $\mathrm{y}$ axis. Further indicated are the needle guide, stereo cameras, projector, LED array and the US probe. 
to the end-effector via a clip and the mechanism can be exchanged with other clips to fit different probe types.

To aid in the localization phase, cameras and a projector are installed. The cameras are a set of wide angle sereo cameras (KYT-U200-SNF01, Kayeton Technology Co.,Ltd, China). Due to the wide angle lenses the cameras see a large area of the breast even when close to the surface. The cameras are synchronized since this is required for accurate stereo vision on a moving frame. They communicate with the PC via a USB2.0 interface. For proper segmentation of the colored markers, some supporting illumination is needed. Therefore, two LED arrays are placed next to the cameras. The projector used for deformation measurements is placed in between the stereo cameras (SK UO Smart Beam, Innoio, S. Korea). It accepts HDMI data input. Utilizing structured light the surface of the breast can be reconstructed.

As discussed, the needle placement is performed by a 3DOF manipulator which consists of two links and one needle guide. Link 1 is $57.39 \mathrm{~mm}$ and link 2 is $50.36 \mathrm{~mm}$. The motors employed in the needle guidance are Herkulex DRS 0201 (DST Robot Co., Ltd, S. Korea) motors. These are smart servos integrating a motor, gear reducer, control circuitry and communication in one package. Its range is $320^{\circ}$ and its resolution $0.325^{\circ}$. Based on these numbers and the forward kinematics of system, the maximum cartesian error for targets in the US plane is expected to be $0.7 \mathrm{~mm} . .1 .0 \mathrm{~mm}$, where the error increases with lesions located further from the needle guide. A workspace analysis is shown in 11.3 .

To integrate all electrical components in the end-effector, a printed circuit board (PCB) has been designed. This PCB integrates a micro controller (MCU) (ESP8266, Espressif Systems, China), supplies for the cameras, the pico projector, and the motor, LED drivers and communication with the robot controller. The MCU is programmed in the Arduino IDE (Arguino AG, Italy) to take serial commands from the robot controller via USB which are then translated to e.g. motor serial commands and LED brightness. A second USB is connected for data transfer with the cameras. The board has separate supplies for the micro controller and the motors. The motor supply contains a relay which is connected to the safety layer of the robot controller (Sunrise cabinet). The system is programmed to shut down the motors in case of an emergency. However, the communication with the end-effector keeps running since the micro controller is connected to a different supply.

All structural parts, e.g. the links and the housing, of the end-effector are are printed by fused deposition modeling printers - A Fortus 250MC (Stratasys, Ltd., USA) and an Ultimaker S5 (Ultimaker, The Netherlands). The materials used are ABS and PLA. 


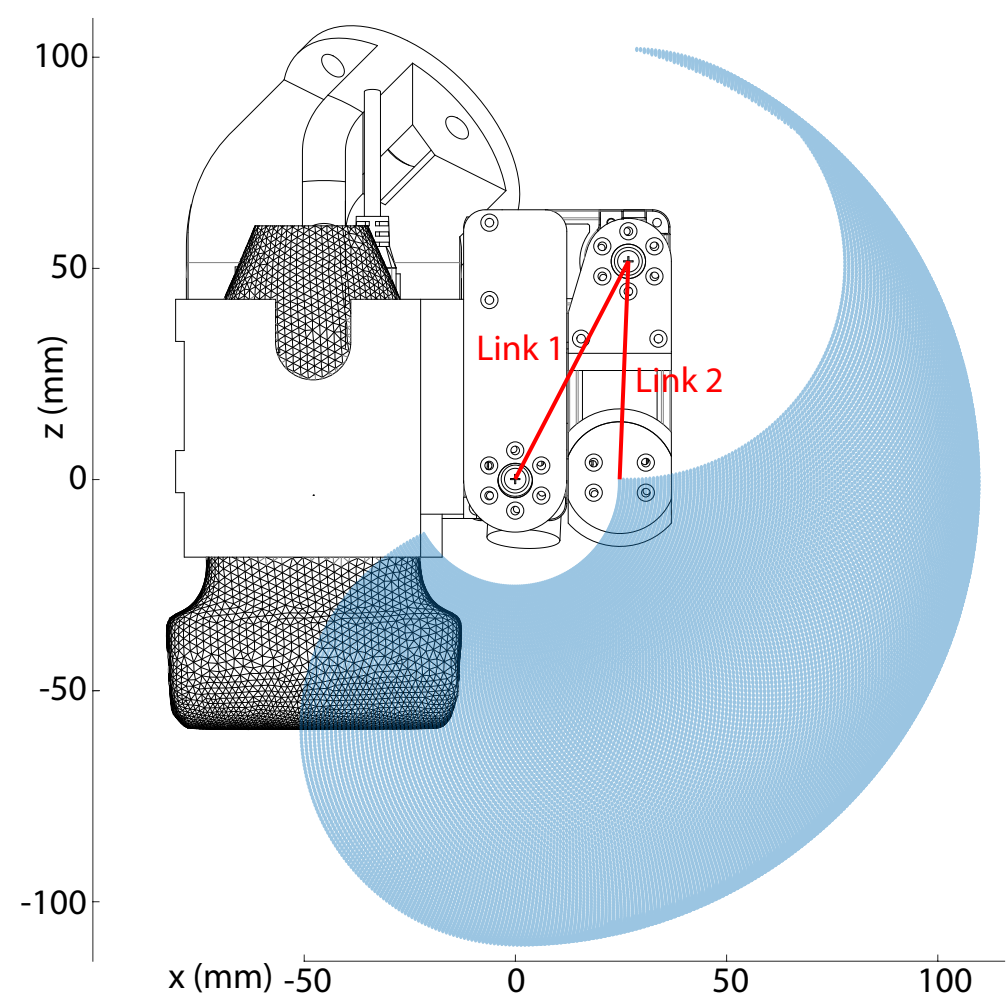

Figure 11.3: Workspace analysis of the 3DOF needle guide. Link 1 is $57.39 \mathrm{~mm}$ and link 2 is $50.36 \mathrm{~mm}$. The origin is located in the joint of the first motor.

\section{Needle stop}

The design of the needle stop is presented in Figure 11.2. The restriction to a $1 \mathrm{D}$ movement in the insertion direction is guaranteed by the entrance and the exit of the needle, which are rings with the needle diameter made of a hard type of plastic (VeroClear, Stratasys, Ltd., USA). This plastic also forms a chamber together with a more flexible plastic (Agilus Black, Stratasys, Ltd., USA), which asides from the entrance and exit forms the remainder of the needle guide. Both plastics are printable utilizing an Objet Eden 260VS (Stratasys, Ltd., USA). The needle is stopped by allowing air pressure to enter the chamber and deform the flexible part of the needle guide. The tube shrinks and causes friction forces which stop the needle. The thickness of the tube has been estimated using $[11,74]$ :

$$
\delta r=\left(\frac{1-\nu}{E}\right)\left(\frac{a^{2} p_{i}-b^{2} p_{o}}{b^{2}-a^{2}}\right) r+\left(\frac{1+\nu}{E}\right)\left(\frac{a^{2} b^{2}\left(p_{i}-p_{0}\right.}{b^{2}-a^{2}}\right) \frac{1}{r}
$$


In which $\delta r$ is the change in radius $[\mathrm{m}]$ due to the difference in pressure on the outside $p_{o}[\mathrm{~Pa}]$ and the inside $p_{i}[\mathrm{~Pa}]$ of the tube, $r$ is the initial radius of the tube $[\mathrm{m}] E$ is the Young's modulus of the material $[\mathrm{Pa}], \nu$ is the Poisson's ratio of the material, $a$ and $b$ are the inner and the outer radius of the tube [m]. It was calculated that a wall thickness of $0.75 \mathrm{~mm}$ for a tube with radius $1.5 \mathrm{~mm}$ should work for pressures in the range of $0 . .6 \times 10^{5} \mathrm{~Pa}$ During insertion, the position of the needle is measured by a laser sensor (PAT9125, PixArt Imaging Inc., Taiwan). This is a sensor which is able to measure displacements of objects located $1 \mathrm{~mm} . .10 \mathrm{~mm}$ from the sensor with a maximum resolution of $1275 \mathrm{cpi}$. The advantage of this sensor is that it is highly accurate and does not touch the needle during measurements. The sensor values are processed by the MCU. Based on these measurements, the controller decides when to open the pneumatic valve (PV3211-24VDC-1/8, FESTO Didactic GmbH \& Co. KG, Germany).
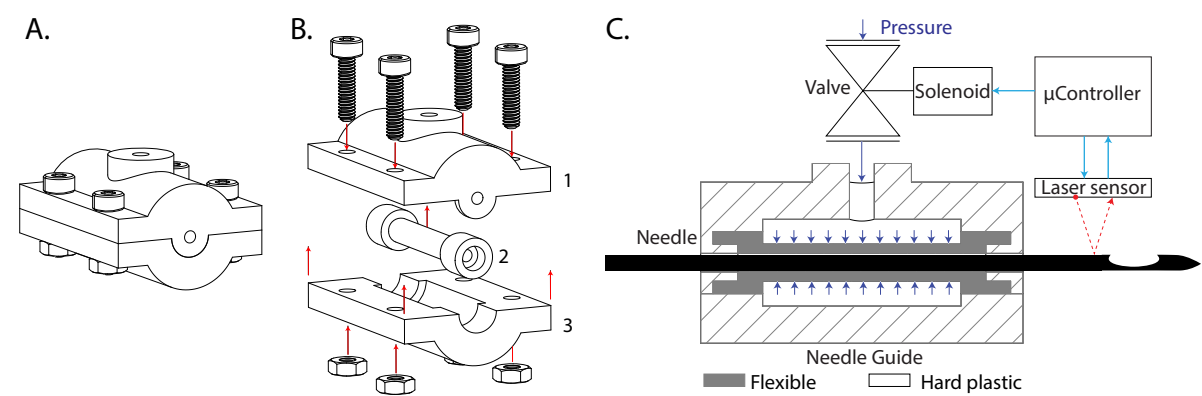

Figure 11.4: A. The needle guide. On the top there is a pressure inlet. The needle fits through the hole in the center. B. An exploded view of the needle stop. Parts 1 and 2 can be printed as an assembly. C. A schematic diagram and a cross section of the needle stop. A laser sensor measures the needle position and communicates this with a micro controller. If the needle reaches the set position, a pressure is applied on the flexible plastic. This creates a friction force on the needle. The micro controller controls the pressure with a solenoid operated valve.

\subsubsection{Experiment}

An experiment was designed to verify the accuracy and precision of the needle guidance system. An experimental setup was fabricated for this (Figure 11.5). The setup consists of a mock-up US probe which is adapted to hold a

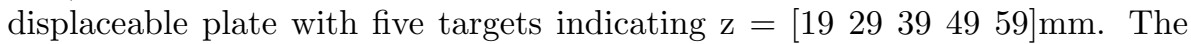
plate itself can be shifted over a rail and fixed on five marked locations with a screw, being $\mathrm{x}=\left[\begin{array}{lllll}-2 & 10 & 0 & 10 & 20\end{array}\right] \mathrm{mm}$. This makes a total of 25 targets (red dots, Figure 11.5). Each target will be approached from seven insertion positions (blue dots, Figure 11.5). For every combination of target and insertion 
position, a needle is inserted until touches the plate. The position on which the needle is in contact with the plate is recorded. A measurement accuracy of $0.5 \mathrm{~mm}$ is achieved by glueing a paper with millimeter grid on the plate. Every combination of insertion and target position will be performed five times. A needle with a conical tip (MRI IceRod ${ }^{T M}$, Galil Medical Inc., USA) is used for this to prevent measurement errors due to the standard bevel shaped tip on a biopsy needle. A MATLAB script (The MathWorks, Inc., USA) is written to command the motor positions to the end-effector and save the measurement values.
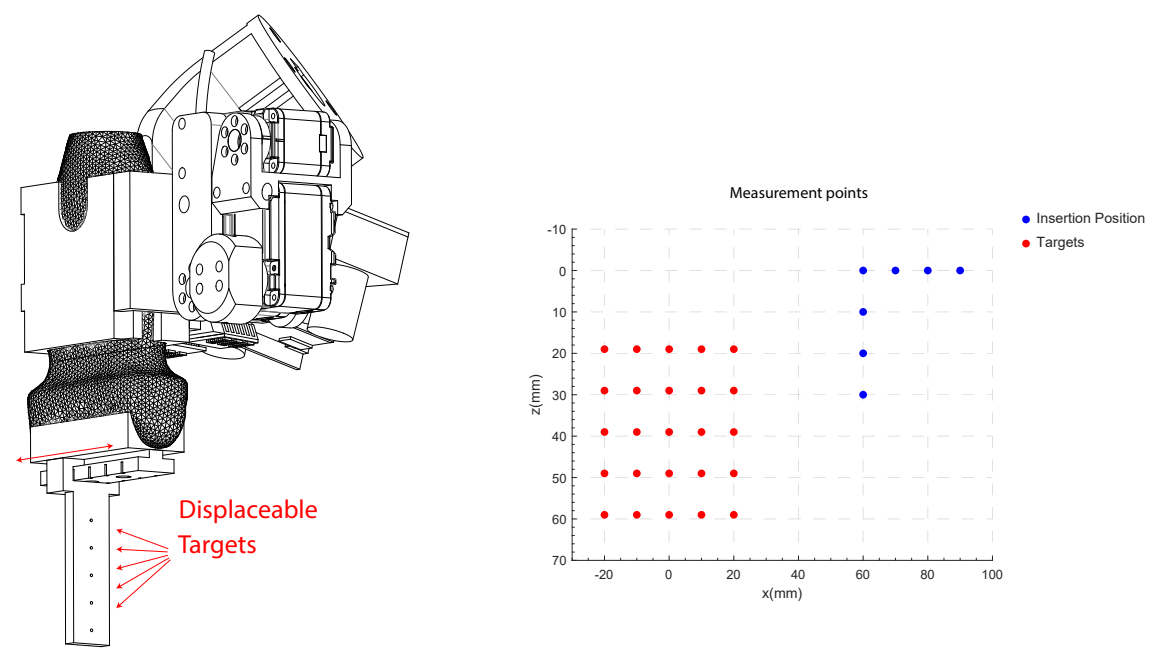

Figure 11.5: Setup for measuring the accuracy and precision of the needle guidance. A displaceable plate with five targets is attached to a mock-up US probe. The plate is placed on five predefined positions. Each target position (red dots) will be targeted from seven insertion positions (blue dots). The positions are commanded and recorded with a Matlab GUI.

\section{Needle stop}

To determine the accuracy and precision of the needle stop, the needle is inserted ten times for different depths, $d_{\text {set }}=\left[\begin{array}{llll}30 & 50 & 70 & 90\end{array}\right] \mathrm{mm}$. The depth at which the needle is stopped, is measured using a micro manipulator which is moved towards the tip of the needle until the sensor of the needle stop measures that the needle has been pushed. This gives a measurement accuracy of approximately $10 \mu \mathrm{m}$. Furthermore, the holding force will be determined for pressures of [2 4 6] bar using a spring balance. 


\subsection{Results}

In Figure 11.6 the produced end-effector is presented. On the left you see the end-effector with red arrows indicating the probe, LEDs, cameras, projector, needle guide and the needle. On the right the end-effector is pictured in its envisioned setup performing a biopsy on a phantom breast.

In Figure 11.7, the results of the experiment are shown. The red dots show the mean position for every target while in blue ellipses are drawn using the standard deviation in $\mathrm{z}$ - and y-direction. Looking at the red dots from the side and the front, it appears the measured points have a bias both in translation and rotation with respect to the targeted positions. This bias can be removed by applying the built-in MATLAB Procrustes analysis to determine the linear transformation between the measurement results and the initially targeted positions. After this bias removal, the mean error in y-direction is $0.03 \pm 0.36 \mathrm{~mm}$, the maximum being $0.1 \mathrm{~mm}$ at target 22 . The mean error in z-direction is $0.3 \pm 1.5 \mathrm{~mm}$, the maximum being $-1.4 \mathrm{~mm}$ at target 5 . If the standard deviations are combined to an ellipse, the largest standard deviation was found at target 25, being $0.47 \mathrm{~mm}$ in y-direction and $1.81 \mathrm{~mm}$ in z-direction. Furthermore, the largest standard deviation in z-direction is found at target 5 , being $3.1 \mathrm{~mm}$.
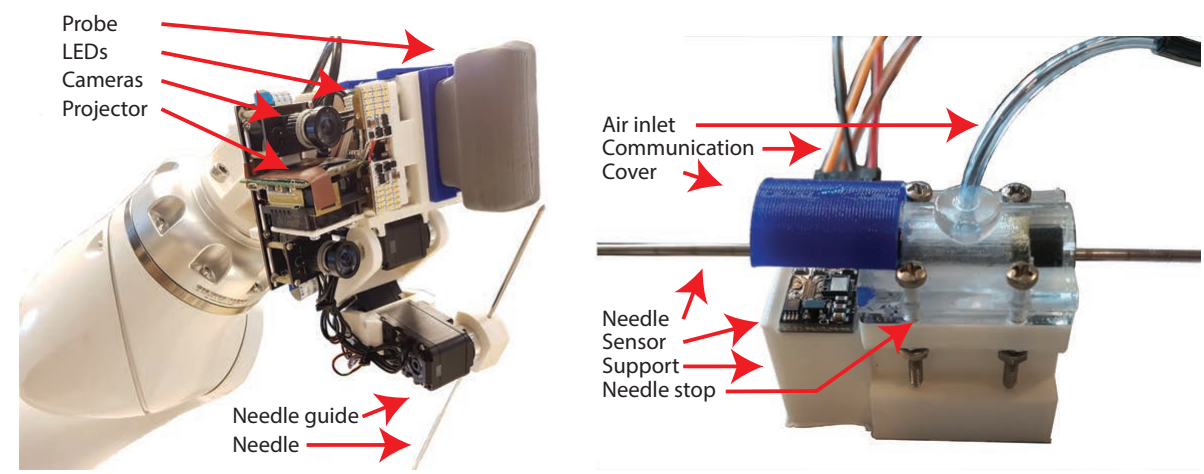

Figure 11.6: Left: The end-effector. Right: The needle stop. Red arrows indicate the relevant parts. 

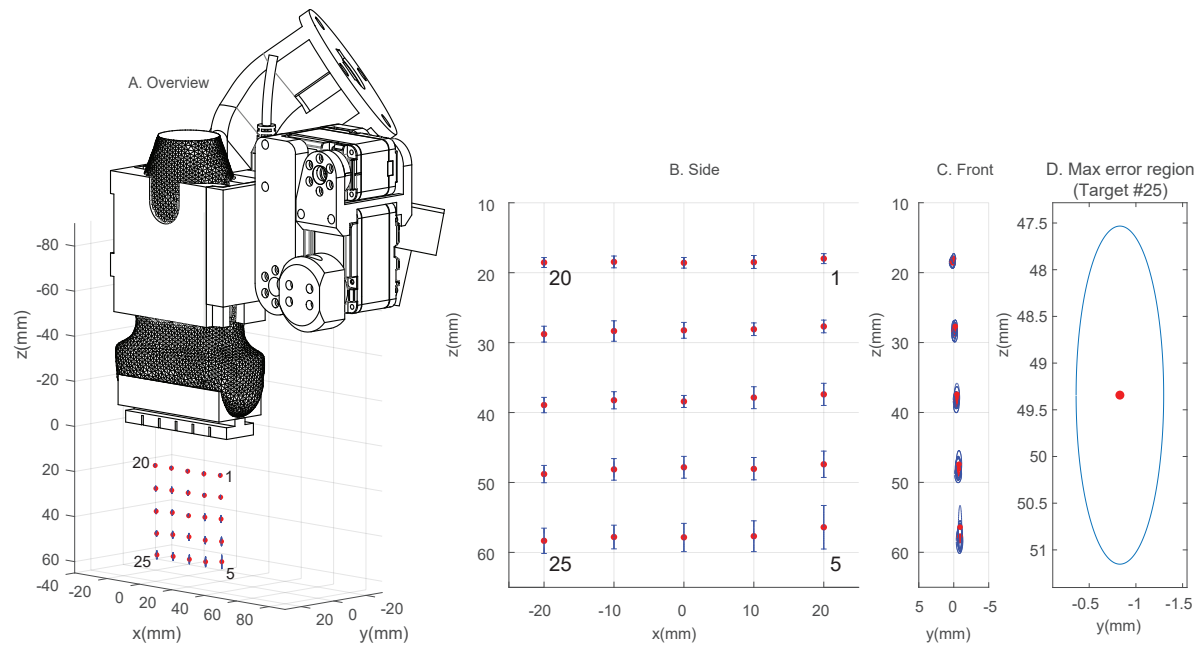

Figure 11.7: A. The measured points plotted together with the end-effector. B. The measured points plotted in the xz-plane. C. The measured points plotted in the yz-plane. D. The position which was targeted the least precise.

In Table 11.1 the results of the needle stop experiment are presented. In these results, the bias of the micro manipulator with respect to the needle guide $(\approx 1.46 \mathrm{~mm})$ has been removed and the resolution of the sensor was corrected to be $\approx 1210 \mathrm{cpi}$ instead of $1275 \mathrm{cpi}$ using the lsline function of MATLAB to plot a least squares line thought the measurement data. The mean error in the tested range was $40 \mu \mathrm{m}$, while the maximum error among all measurements was $1.18 \mathrm{~mm}$. The holding force was determined to be $3.5 . .6 \mathrm{~N}$.

Table 11.1: Top: The set and measured needle depths. Bottom: The applied pressure and the corresponding holding force.

\begin{tabular}{|c||cccc|}
\hline Set $[\mathrm{mm}]$ & 30 & 50 & 70 & 90 \\
\hline \hline Measured avg. [mm] & 30.0066 & 50.0293 & 69.9217 & 90.0424 \\
\hline Min [mm] & 29.7828 & 49.5820 & 69.0870 & 89.8091 \\
\hline Max [mm] & 30.2646 & 51.1846 & 70.3244 & 90.3568 \\
\hline
\end{tabular}

\begin{tabular}{|c||ccc|}
\hline Pressure [bar] & 2 & 4 & 6 \\
\hline \hline Hold force [N] & 3.5 & 5 & 6 \\
\hline
\end{tabular}




\subsection{Discussion}

An end-effector for a robotic arm has been designed which is utilized in all the steps of a robotic assisted guided breast biopsy such as registration, 3D volume acquisition and the US guided biopsy. The presented end-effector integrates all necessary features in a small package: The $45^{\circ}$ angle of the US probe w.r.t. the flange allows the robot to reach the breast near the chest wall during both the scanning and the biopsy phase. The cameras and pico-projector enable registering of pre- and intra-operational data. A 3DOF needle guide assists the radiologist in targeting the right location during the biopsy procedure. All electronics are connected to a small electrical board which communicates with the robot controller. Furthermore, a needle stop has been presented which stops and holds the needle at a specified depth.

The focus of the experiments was on needle placement accuracy. The aim was to reach lesions with a size of $4 . .10 \mathrm{~mm}$ in the US image plane. Using a 3DOF needle guide with three Herkulex motors the expected accuracy was $0.7 . .1 .0 \mathrm{~mm}$. The biopsy accuracy reported from the experiments is $0.03 \pm 0.36 \mathrm{~mm}$ and $0.3 \pm 1.5 \mathrm{~mm}$ in the $\mathrm{y}$ - and z-direction, respectively. This accuracy has the same order of magnitude as anticipated and is satisfactory to perform biopsies on lesions with sizes in the given range. Furthermore, the accuracy is in the same range as results in cited studies $(0.25 . .3 .44 \mathrm{~mm}[126,131])$. The needle stopping accuracy is $40 \mu \mathrm{m}$. This is a higher accuracy than in comparable studies. The maximum holding force of around $6 \mathrm{~N}$ is enough to stop the biopsy needle during a procedure.

Considering the results in Figure 11.7 it is noteworthy that the standard deviations are large with respect to the mean errors. This can be explained by the fact that the motors have backlash in the gears. Also, many of the end-effector's parts are printed with plastics, which results in parts which are less rigid than e.g. metal parts. Furthermore, target 5 has a relatively large standard deviation in the z-direction while being relatively close the the needle guide. This is because the needle is directed at this target under a sharp angle. Small deviations in target placement and the insertion angle cause a relatively large variation in cartesian errors of the needle tip with respect to the target. Furthermore, the largest combined standard deviation is found at target 25 . This is as expected since this target is located the farthest away from the needle guide.

The main limitation of the presented system is in the fact that prototyping techniques are used. Therefore, parts are less rigid and this decreases the precision. Furthermore, the material of the clamping mechanism of the needle stop is too brittle. Therefore, it difficult to make the mechanism airtight and durable. This is especially emerges when applying forces on the needle while the stop is activated.For clinical application the design of the needle stop could 
still be improved on some aspects: While the needle is being clamped, it still has a bit of play in the direction of insertion due to the elastic nature of the clamping material. However, this can be accounted for with other fabrication methods.

The needle guide has several advantages: the radiologist remains in control yet has the biopsy accuracy of a robot. Due to the frictionless movement in the insertion direction, the physician still has the feedback when puncturing the skin and other tissue boundaries. The laser sensor measures the needle position accurately without touching the needle. The fact that the needle measures displacements instead of position does not influence the accuracy in the tests performed. Because the laser is at the distance of the needle, the needle guide can easily be replaced after a biopsy is performed or if another diameter guide is needed. Furthermore, the system works independently of the length of the needle. In case of a power down the needle is simply released and in case of emergency the practitioner could remove the needle with minor effort by overcoming the clamping forces.

\subsection{Conclusion and recommendations}

This paper introduces an end-effector that enables a robotic arm to autono[]mously scan the breast using US and assists the radiologist in performing the US guided biopsy.

The results of this study are an example of how to integrate different aspects of robotic US scanning and robotic assisted biopsy in one functional device. The cameras and pico projector are used to combine pre- and intra-operational data. The probe placement under an angle allows the robot to optimally utilize the space during both US scanning and the biopsy phase. The 3DOF needle guide with needle stop assists the radiologist yet is passive in the sense that needle actuation is still not robotized.

The accuracy and precision of the $3 \mathrm{DOF}$ needle guide were determined experimentally. The needle placement was found to be accurate enough to target lesions with a size range of $4 . .10 \mathrm{~mm}$.

The following improvements are recommended to bring the system to clinical practice. Backlash-less motors should be installed to increase the precision and stability. The design of the needle stop could be adjusted such that when the stop is activated the minor movements in the insertion direction are further restricted. Changing the 3D printed plastics for more rigid CNC machined parts will ensure the rigidity of the system and ease sterilization of the parts. Real time communication between the end-effector and the robot can be established to accommodate the system for clinical operations. 


\section{ANALYTICAL DERIVATION OF ELASTICITY IN BREAST PHANTOMS FOR DEFORMATION TRACKING}

V. Groenhuis, F. Visentin, F. J. Siepel, B. M. Maris, D. Dall'alba, P. Fiorini, and S. Stramigioli. Analytical derivation of elasticity in breast phantoms for deformation tracking. International Journal of Computer Assisted Radiology and Surgery, 13(10):1641-1650, 2018 
Ch 12: Analytical derivation of elasticity in breast phantoms for deformation

\subsection{Abstract}

PURPOSE: Patient-specific biomedical modeling of the breast is of interest for medical applications such as image registration, image guided procedures and the alignment for biopsy or surgery purposes. The computation of elastic properties is essential to simulate deformations in a realistic way. This study presents an innovative analytical method to compute the elastic modulus and evaluate the elasticity of a breast using magnetic resonance (MRI) images of breast phantoms.

METHODS: An analytical method for elasticity computation was developed and subsequently validated on a series of geometric shapes, and on four physical breast phantoms that are supported by a planar frame. This method can compute the elasticity of a shape directly from a set of MRI scans. For comparison, elasticity values were also computed numerically using two different simulation software packages.

RESULTS: Application of the different methods on the geometric shapes show that the analytically derived elongation differs from simulated elongation by less than $9 \%$ for cylindrical shapes, and up to $18 \%$ for other shapes that are also substantially vertically supported by a planar base. For the four physical breast phantoms, the analytically derived elasticity differs from numeric elasticity by $18 \%$ on average, which is in accordance with the difference in elongation estimation for the geometric shapes. The analytic method has shown to be multiple orders of magnitude faster than the numerical methods.

CONCLUSION: It can be concluded that the analytical elasticity computation method has good potential to supplement or replace numerical elasticity simulations in gravity-induced deformations, for shapes that are substantially supported by a planar base perpendicular to the gravitational field. The error is manageable, while the calculation procedure takes less than one second as opposed to multiple minutes with numerical methods. The results will be used in the MURAB (MRI and Ultrasound Robotic Assisted Biosy) project.

\subsection{Introduction}

Screening and staging of breast cancer for diagnosis and subsequent treatment is based on medical images acquired on different acquisition modalities and includes mammography (x-ray), ultrasound (US), and MRI.

After image acquisition, proper localizaton of the tumour is essential for biopsy procedures to take tissue samples or to remove the tumour during surgery. To take full benefit from the previously acquired medical images the location of the tumour should be aligned from the pre-operative imaging into the operating room. The position of the patient can vary from prone during MRI scanning to supine position required for breast surgery for example. Dur- 
ing ultrasound scanning and ultrasound guided biopsy, the patient is returned on her back and additional compression is induced by the ultrasound probe. The computation of the elastic properties will serve as input for realtime adjustments of realistic deformations between pre-operative and intra-operative images. For effective deformation models, the elasticity of the model needs to be known with good accuracy, i.e. the difference between computed and actual elasticity must be small. In this study, we aim for a maximum difference in the order of $10 \%$, or at most two times the elasticity variation among FEM-simulated elasticity values. Image registration techniques based on image intensities could be used for small deformations [108], but do not work in cases with large deformations such as the alignment from prone to supine configurations [12].

Deformation of the breast occurs due to body movements. Various physicsbased numerical procedures have been presented for biomechanical modelling and soft tissue deformation. The most common computational schemes are based on linear or nonlinear biomechanical models including mass-spring methods (MSM) [6, 23, 87, 107], the mass-tensor method [33, 104], the boundary element method $[43,71]$ and conventional finite element modelling (FEM) $[9,111,112]$.

In an MSM system, an object is modelled by a collection of point masses linked together with massless springs.

Recent studies show the use of FEM to align data with large deformations of the breast $[63,64]$. In FEM, a body is subdivided into a set of finite elements (e.g. tetrahedral or hexahedra in 3D, triangles or other polygons in 2D). Displacements and positions of each element are approximated from discrete nodal values using interpolation functions:

$$
\phi(x)=\sum_{i} h_{i}(x) \phi_{i}
$$

where $h_{i}$ is the interpolation function for the element containing $x$ and $\phi_{i}$ is the scalar weight associated with $h_{i}$. Different choices for the element type and the interpolation functions exist, which depend on the accuracy requirements, geometry of the objects and computational complexity [85]. In general FEM is used to solve a dynamic problem, which is expressed as partial differential equations (PDEs). These PDEs are then approximated with FEM. The FEM procedure has the advantage that it can handle complicated geometries (and boundaries) of high quality. A dataset of radiological 3D images of the breast anatomy (computed tomography (CT) or MRI) are required to generate a patient-specific FEM. An advantage of MRI is that it shows high sensitivity for detecting breast tumours [29]. The main FEM steps include: tissue classification/segmentation, tissue surface reconstruction, FEM volumetric mesh generation and tissue type assignment for the FEM mesh. 
Ch 12: Analytical derivation of elasticity in breast phantoms for deformation

A patient specific biomechanical model [35] was presented before to provide an initial deformation of the breast before registration between prone and supine MRI images. A zero-gravity reference state for both prone and supine configurations was estimated. The patient-specific unloaded configuration was obtained [36]. The biomechanical methods serve in most cases for the initialization of intensity-based image registration techniques, as in [31] or [31].The sliding motion of the breast on the chest wall was observed [21], but usually a fixed muscle surface is applied during the FEM simulations [62, 80, 101].

This study introduces a method to analytically derive the elastic modulus of the breast from a pair of MRI scans, taking local differences in tissue density and elasticity into account. The two MRI scans differ by the direction of the gravitational field, which are opposite to each other. Contrary to FEM-based numerical simulations, it is not needed to convert the MRI scan into a volumetric mesh, so mechanical properties on voxel scale are preserved. Also, only one iteration over all voxels is necessary, which makes the method relatively fast

The proposed analytical method requires the breast to be vertically supported by a rigid planar base. As the rib cage is approximately cylindrical, a human breast would need to be supported by a patient-mounted flat plate with a hole for the breast. In an MRI scanner, the breast coil could serve this purpose.

To avoid introduction of significant non-gravity-induced deformations when converting from prone to supine position, it is desirable to use a patient rotation system (PRS) that allows leaving the patient on the bed with breast coil attached, while being flipped over by $180^{\circ}$. Such a system has been developed previously by Whelan et al. [132], which theoretically could be used to take MRI scans of a planar supported breast in both prone and supine position. It may also be possible to tilt certain MRI scanners such as the $0.25 \mathrm{~T}$ G-scan Brio (Esaote SpA, Genua, Italy), although this is generally limited to rotation over $90^{\circ}$ only.

\subsection{Materials and Methods}

Four breast phantoms were constructed (Figure 12.1, right), consisting of a rigid base with three fiducials, stiff superficial tissue, soft deep tissue and 3-4 lesions.

The superficial and deep tissues and lesions were made of polyvinyl chloride (PVC) with plasticizer mixed in different ratios to obtain different stiffnesses. Contrary to gelatin-based phantoms, PVC is a durable material that can stay intact for extended periods. The superficial tissue consists of relatively stiff PVC which was shaped using a pair of moulds (Figure 12.1, left) and afterwards filled with soft PVC to mimic deep tissue. The lesions were cut in different sizes 

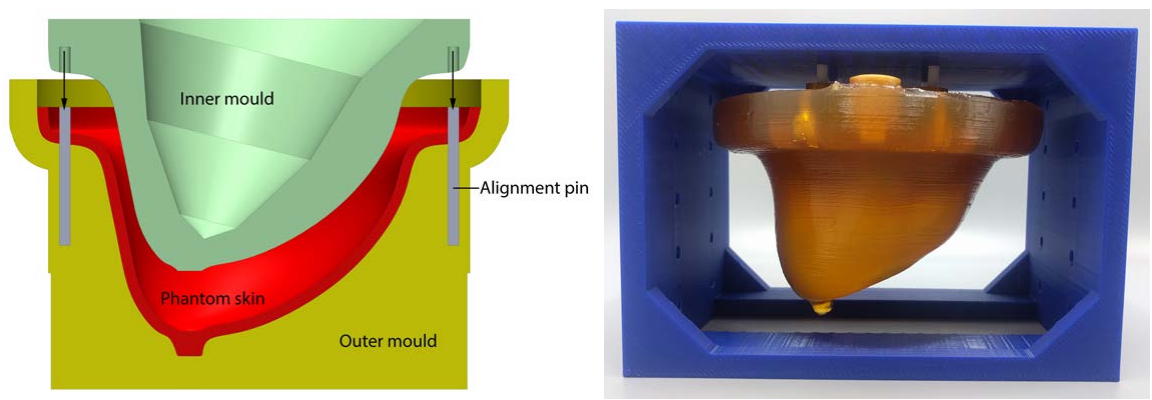

Figure 12.1: Left: pair of moulds (yellow, green) for manufacturing superficial tissue (red). Right: one PVC breast phantom mounted in prone position.

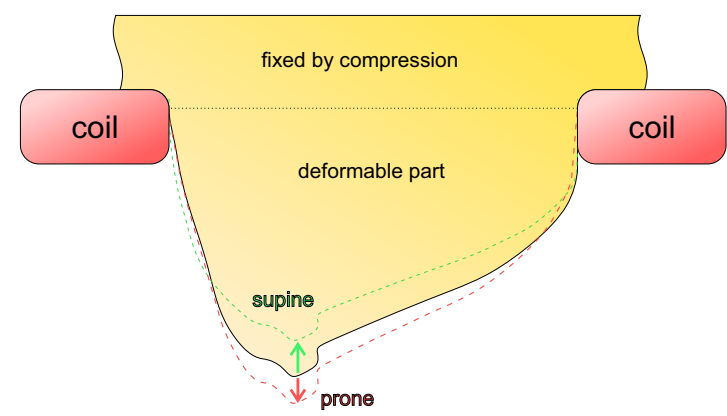

Figure 12.2: Breast in coil, with gravity-induced deformations in prone and supine positions (dashed lines).

and shapes from a block of relatively stiff PVC, placed inside the deep tissue at random locations. A rigid frame was put on top and covered with a layer of stiff PVC. The four phantoms which were manufactured this way, differ only in the stiffness of deep tissue and the placement of lesions.

Figure 12.2 shows the outline of a breast phantom in a neutral reference state. Depending on the orientation (prone or supine), it is deformed by the gravitational field and tip is displaced towards the anterior or posterior direction. The magnitude of these deformations is related to the elasticity, and the approach of the research is to reconstruct the elasticity from these deformations using different methods.

The base represents a rigid inertial frame, which must be planar and normal to the gravitational direction. While a patient's rib cage provides a rigid supportive base, it is not planar but approximately cylindrical. An external structure such as a breast coil (Figure 12.2) may be required to provide this planar support.

Each of the four phantoms was scanned in a $0.25 \mathrm{~T}$ MRI scanner (G- 
Ch 12: Analytical derivation of elasticity in breast phantoms for deformation
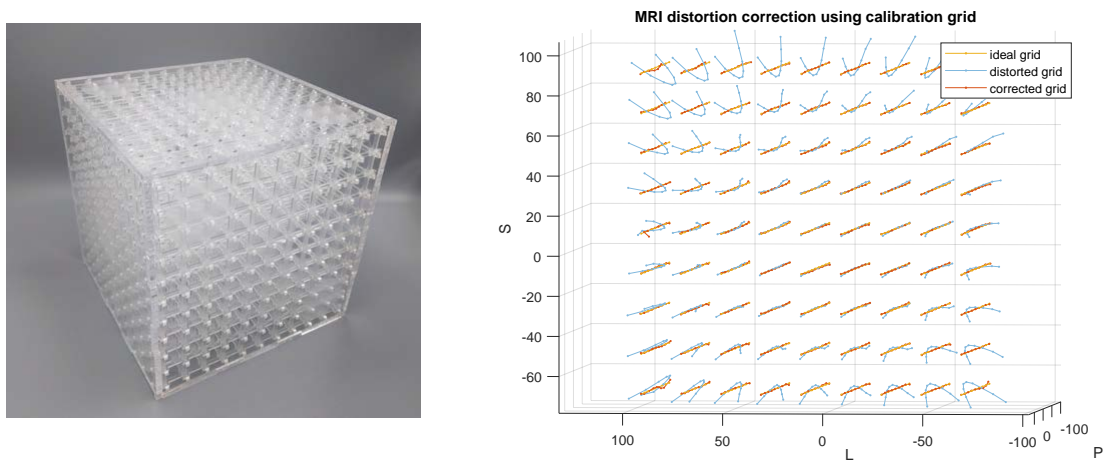

Figure 12.3: Left: MRI calibration grid. Right: actual (yellow), observed (blue) and distortion-corrected (red) grid locations of the calibration cube.
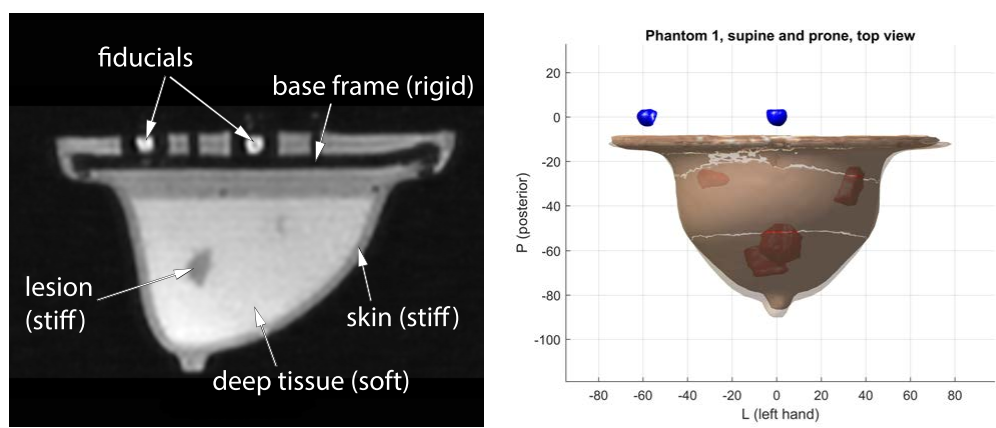

Figure 12.4: Left: Example sagittal MRI slice. Right: Phantom I in prone and supine configuration, superimposed.

Scan Brio) using the 3D balanced steady-state free precession (bSSFP) sequence, with parameters $\mathrm{TR}=10 \mathrm{~ms}, \mathrm{TE}=5 \mathrm{~ms}, \mathrm{FA}=60^{\circ}$, acquisition resolution $1.5 \times 1.8 \times 2.0 \mathrm{~mm}$ and isotropic reconstruction resolution $0.94 \mathrm{~mm}$.

The scanner was previously calibrated using a custom 3D calibration grid (Figure 12.3, left) from which a fifth order correction polynomial correction function was constructed. The ideal, distorted and corrected grid patterns are shown in Figure 12.3. The measured residual error is $0.2 \mathrm{~mm}$, so sub-pixel resolution is feasible.

The distortion-corrected MRI scans (Figure 12.4, left) were segmented by intensity thresholding and automatically aligned with a rigid transformation using the three fiducials, in which the root-mean-square registration error was found to be $0.2-0.3 \mathrm{~mm}$. From these data, surface and volumetric meshes in different levels of detail were constructed.

Figure 12.4, right shows two configurations of phantom I, overlaid on each 
other, after segmentation and registration. A significant displacement of the tip resulting from the change in gravity field direction can be observed.

\subsection{Elasticity estimation}

\subsubsection{Preamble}

The deformation of an object in a gravitational field is the result of elongations of tissue, which depends on the local ratio of tensile stress $\sigma$ and Young's modulus $E$ :

$$
\epsilon=\frac{\sigma}{E}
$$

The stress at a given location is primarily induced by the weight of the masses below that location, and also influenced by interactions with surrounding tissue. In the general case, the resulting stress distribution in the object is a complex pattern and cannot be solved analytically, requiring simulations to quantify the deformations. However, in our case we can use the knowledge that the object's attachment to the rigid frame is planar and perpendicular to the gravity direction, when in prone and supine positions. For objects with a constant cross-section such as a block or a cylinder, it can be shown (see section 12.4.2) that the deformation displacement can be solved analytically.

We introduce the assumption that the tensile stress $\sigma$ solely depends on the vertical position in the object, i.e. it is constant within any planar cross-section parallel to the base. It can be shown that this assumption is valid for blocks, cylinders and prism-shaped objects which have a constant cross-section. For the breast phantom shapes, the assumption can be justified by the fact that the masses of the whole breast are substantially positioned below the rigid base. To validate this assumption, the stress distribution and elongation for a range of geometric shapes are also investigated.

\subsubsection{Analytical derivation of elasticity}

Figure 12.5 schematically shows the forces and pressures acting on a shape with inhomogeneous density and elasticity, hanging from a planar, rigid attachment on the top. At a given height $h$, the cross-sectional area is $A(h)$, the mass of the body below it is denoted as $m(h)$ and the gravitational force acting on it $F(h)$. We now derive expressions for the vertical stress $\sigma(h)$ and elongation $\epsilon(h)$ for every height, leading to a formula for the displacement $D$ of the lower extremity of the body.

The total mass of the body up to height $h$ is given as: 
Ch 12: Analytical derivation of elasticity in breast phantoms for deformation 232 tracking

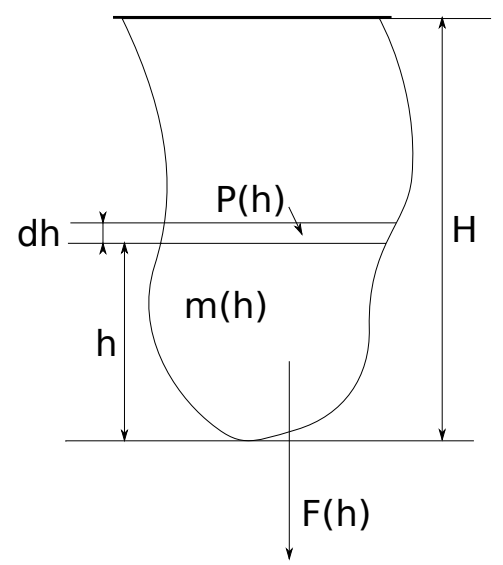

Figure 12.5: Schematic view of force and pressure at a given height

$$
m(h)=\int_{0}^{h} \iint \rho(x, y, z) d x d y d z
$$

The gravitational force acting on the slice at height $h$ is calculated as:

$$
F(h)=m(h) g
$$

The tensile stress in the slice is generally not constant and its exact distribution depends on many levels of tissue interactions. We are interested in the mean tensile stress $\bar{\sigma}(h)$, which is found by dividing the gravitational force by the slice's cross-sectional area:

$$
\bar{\sigma}(h)=\frac{F(h)}{A(h)}=\frac{m(h) g}{A(h)}
$$

The tissue elasticity is also inhomogeneous in general, with local Young's modulus $E(\vec{r})$, again averaged to $\bar{E}(h)$ for height $h$. The local relative elongation is $\epsilon=\Delta L / L_{0}=\sigma(\vec{r}) / E(\vec{r})$, and the mean elongation at height $h$ is given as:

$$
\overline{\epsilon(h)}=\frac{\bar{\sigma}(h)}{\bar{E}(h)}=\frac{m(h) g}{A(h) \bar{E}(h)}
$$

The total displacement of the body's lower extremity is found by integrating all infinitesimal elongations:

$$
D=\int_{0}^{H} \bar{\epsilon}(h) d h=g \int_{0}^{H} \frac{m(h)}{A(h) \bar{E}(h)} d h
$$


The purpose of this study is to find the average Young's modulus $E$ from a pair of gravity-induced body displacements. To preserve differences in (mean) elasticity among slices, we factorize every slice's elasticity into a constant factor $E$ and a layer-specific adjustment factor $\hat{E}(h)$ :

$$
\bar{E}(h)=E \hat{E}(h)
$$

The displacement equation can now be written as follows:

$$
D=\frac{g}{E} \int_{0}^{H} \frac{m(h)}{A(h) \hat{E}(h)} d h
$$

It can split into an object-specific intrinsic part which remains constant across all simulations, and an extrinsic (variable) part depending on $g$ and $E$ only. The intrinsic part $\beta$ is defined as:

$$
\beta=\int_{0}^{H} \frac{m(h)}{A(h) \hat{E}(h)} d h
$$

Substituting into $D$ gives:

$$
D=\beta \frac{g}{E}
$$

For the scanned breast phantoms, we therefore assume that the displacement (for small displacements) is linear in $g / E$, with proportionality factor $\beta$. The $\beta$ value can be estimated from DICOM data, in combination with knowledge of the materials. For PVC phantoms, its density was measured to be $\rho=1.075 \mathrm{~g} / \mathrm{cm}^{3}$.

Analyzing the prone and supine scans of a phantom, we have $\beta_{p}$ and $\beta_{s}$ for prone and supine, respectively. In general, $\beta_{p} \neq \beta_{s}$, because the shapes are significantly different: the total volume and cross-sectional area at the base are approximately equal, but due to difference in height the cross-sectional shape is more squeezed in prone position than in the supine one.

The phantom height $H$ is ill-defined due to possible irregularities at the tip, but the difference $\Delta H=H_{p}-H_{s}$ can be accurately determined by comparing point clouds around the tip using e.g. the iterative closest point algorithm [14], and optimizing $\Delta H$ such that the total point distance is minimal, or alternatively by comparing the centroids of the point clouds.

The parameter we want to compute is the Young's modulus $E$. When no forces act on the phantom, it would have some shape halfway the prone and supine shapes. The tip displacement to either prone or supine shape in a gravitational field $g$, is $\Delta H / 2$. We can now derive the Young's modulus $E$ as follows:

$$
\beta_{n}=\frac{\beta_{p}+\beta_{s}}{2}
$$


Ch 12: Analytical derivation of elasticity in breast phantoms for deformation

$$
E=\frac{\beta_{n} g}{\Delta H / 2}=\frac{2\left(\beta_{p}+\beta_{s}\right) g}{\Delta H}
$$

\subsubsection{Numerical simulation of deformations}

The purpose of FEM simulations is to determine the elasticity $E$ of the different phantoms, based on the segmented models. The general strategy is to apply a gravitational field to the FEM model of a phantom in a specific direction. This deformed model is then compared to a reference phantom which was scanned in a different orientation, providing information about the elasticity parameter.

In the following subsections we present two strategies to find the Young's modulus by simulation, of which one strategy is performed by two different simulation software packages.

\section{Estimating the $\beta$ values by simulation in SOFA}

In subsection 12.4.2, we have introduced a method to derive the values of $\beta$ for the four phantoms in different orientations directly from a DICOM scan. In this section, we find $\beta$ by simulation in SOFA at five different mesh resolutions [39]. For each mesh resolution, we have run a simulation with the phantom's Young's modulus set to $E=6000 \mathrm{~Pa}$ and gravity $g=2.0 \mathrm{~m} / \mathrm{s}^{2}$. After 100 iterations, the simulation has stabilized and the vertices of the mesh in this configuration were extracted and analyzed. The displacement from the initial position follows by comparing the point clouds around the tip. The value of $\beta$ then follows from equation (12.10). This procedure is repeated for each resolution of the mesh and for both prone and supine orientations, then the mean $\beta_{s}$ and $\beta_{p}$ values were computed. From the $\beta_{s}, \beta_{p}$ and $\Delta H$, and assuming linearity of the displacement to $g / E$ ratio, the Young's modulus $E$ can be derived using equations (12.11) and (12.12).

\section{Supine-prone and prone-supine simulation and matching in SOFA and Febio}

Taking a phantom scanned in supine configuration, the base of the phantom is immobilized and a force field sized two times the gravity $\left(19.62 \mathrm{~m} / \mathrm{s}^{2}\right)$ in anterior direction is applied to the phantom. After stabilization in simulation, the final state is extracted and compared to the phantom in prone position, which serves as the reference phantom.

The error value, $\epsilon$, is defined as the distance between the simulated and reference phantoms in the area around the tip of the breast and can be positive or negative. The actual value is dependent on the elasticity parameter $E$ of the phantom, which is optimized to bring $\epsilon$ to zero. 


\begin{tabular}{c|c|c} 
geometric shape & calculated $\beta$ & simulated $\beta$ \\
\hline a & 2375 & 2169 \\
b & 2373 & 2229 \\
c & 772 & 724 \\
d & 1638 & 1581 \\
e & 4500 & 4979 \\
f & 213 & 205 \\
g & 1932 & 2276 \\
h & 3802 & 3797 \\
i & 4942 & 26499 \\
\hline
\end{tabular}

Table 12.1: Calculated and simulated $\beta$ values for the nine geometric shapes.

The minimization is performed using the Newton's method computed over $\mathrm{E}$ and the distance error, corrected by an adaptive step approach (when the FEM analys software diverge). When procedure ends, i.e. when the method achieves a pre-defined error or when it reaches a maximum number of iterations, the estimated $\mathrm{E}$ parameter is returned with its associated error.

The procedure is then repeated for the opposite direction (prone to supine). In general, this also leads to a different $E$ value. The mean value (squareharmonicmean-root) of $E_{s p}$ and $E_{p s}$ is then taken as the elasticity of the final phantom.

\subsection{Results}

\subsubsection{Validation of analytical stress calculation on geo- metric shapes}

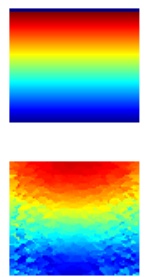

a
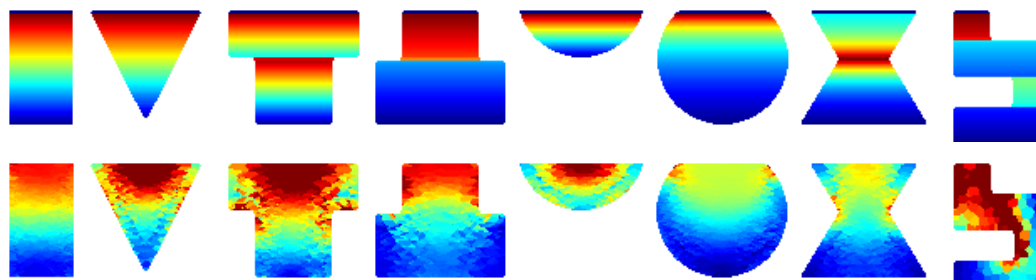

b

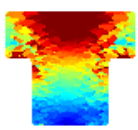

d

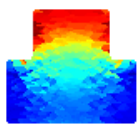

e
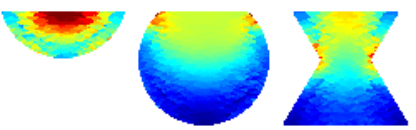

h

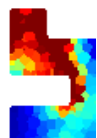

g

$100 \%$

Figure 12.6: Analytically derived tensile stress (top row) compared with simulated stress (bottom row) for a selection of geometric shapes. 


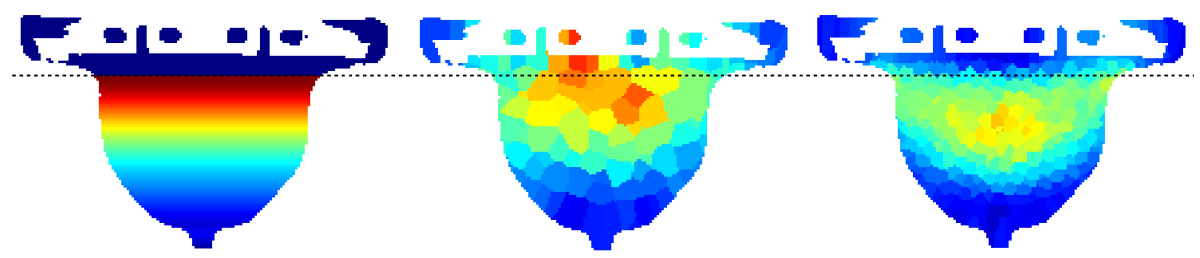

Figure 12.7: Tensile stress for phantom I in the transversal plane, in supine position. Left: derived using analytical method. Center and right: numerically simulated using SOFA in low resolution (center) and high resolution (right). The dashed line indicates the boundary plane between the rigid and deformable parts.

Nine homogeneous geometric shapes were generated and analyzed: two cylinders with different aspect ratios, a cone, a T-piece in normal and upsidedown orientation, a half sphere, a sphere, an hourglass and a snake-like shape.

Figure 12.6 shows the stress distribution along the vertical mid-way plane for all nine shapes. The first row uses the analytical computation method. The assumption that the stress distribution is constant in a cross-sectional area parallel to the base, is reflected in having constant colors in horizontal direction. The second row shows the tensile stress from numerical simulations using the SOFA software package under the same conditions.

Table 12.1 lists the calculated and simulated $\beta$ values for the same geometric shapes.

The following observations can be made:

- For cylinder, cubic and prism-like shapes that have a constant crosssectional area ( $\mathrm{a}$ and $\mathrm{b}$ ), the numerically derived stress distribution matches the analytically derived one quite well. The $\beta$ values derived by both methods are well comparable (deviation under 9\%).

- For shapes that do not have a constant cross-sectional area, but are substantially vertically supportive (c-h), the analytically calculated and SOFA-simulated $\beta$ values are still comparable (deviation up to $18 \%$ ) although the stress distribution is different.

- For shapes in which the lower extremity is not vertically supported by the base, i.e. no vertical line of maximum height can be drawn that entirely lies within the model (i), both the analytically calculated $\beta$ value and the stress distribution are inconsistent with simulations.

\subsubsection{Analytical derivation of elasticity of phantoms}

Each of the four phantoms was scanned in prone and supine position, and from the resulting DICOM scans the $\beta_{p}$ and $\beta_{s}$ values were computed using 


\begin{tabular}{c|c|c|c|c} 
phantom & $\beta_{s}$ & $\beta_{p}$ & $\Delta H$ & $E$ \\
\hline I & 1215 & 1298 & 3.28 & 7514 \\
II & 1129 & 1269 & 4.73 & 4972 \\
III & 1356 & 1444 & 3.58 & 7673 \\
IV & 1420 & 1471 & 2.93 & 9677
\end{tabular}

Table 12.2: Analytically derived properties of four phantoms, under the assumption of constant tensile stress in each cross-section.

\begin{tabular}{c|c|c|c|c} 
phantom & $\beta_{s}$ & $\beta_{p}$ & $\Delta H$ & $E$ \\
\hline I & $1007 \pm 58$ & $1134 \pm 38$ & 3.28 & $6403 \pm 207$ \\
II & $947 \pm 41$ & $1125 \pm 36$ & 4.73 & $4297 \pm 113$ \\
III & $1131 \pm 48$ & $1259 \pm 61$ & 3.58 & $6549 \pm 213$ \\
IV & $1170 \pm 43$ & $1383 \pm 34$ & 2.93 & $8548 \pm 184$
\end{tabular}

Table 12.3: Properties of four phantoms, derived by numerical simulation in SOFA in five different resolution scales and then averaged.

Equation 12.9 and assuming a homogeneous density and elasticity distribution. From these values plus the observed vertical displacements, the $E$ parameters were computed using Equation 12.12 and the results are listed in Table 12.2. It can be observed that phantom IV has the highest $\beta$ and $E$ values, making it the stiffest phantom, while phantom II is the softest one. In general, the $\beta$ values are higher in prone position, which is as expected.

\subsubsection{Simulation of $\beta$ in SOFA}

For numerical FEM simulations, each DICOM scan was segmented and meshed at five different levels of detail and subsequently simulated in the SOFA simulation package. The resulting $\beta$ values of the four phantoms (in both orientations) plus the averaged $E$ value, are listed in Table 12.3. Calculation of each $\beta$ value requires ten simulation runs in SOFA, lasting a few minutes in total.

Figure 12.7 shows the analytically-derived stress distribution in the transversal plane of phantom I in supine configuration together with the numerically simulated stress distribution in the same plane at low and high resolutions. It can be observed that the resulting stress patterns are comparable to that of certain geometric shapes in Figure 12.6 (a-h). Only the analytic method shows a sharp transition at the boundary layer, as the analytical method uses slices with thickness of one voxel while the FEM-based method subdivides the volume in a different way. 
Ch 12: Analytical derivation of elasticity in breast phantoms for deformation 238 tracking

\begin{tabular}{c|c|c|c} 
phantom & $E_{s p}$ & $E_{p s}$ & Mean $E$ \\
\hline I & $5047 \pm 374$ & $6459 \pm 373$ & $5688 \pm 272$ \\
II & $3395 \pm 189$ & $4513 \pm 272$ & $3895 \pm 159$ \\
III & $5381 \pm 376$ & $6828 \pm 438$ & $6040 \pm 288$ \\
IV & $6245 \pm 433$ & $7445 \pm 322$ & $6805 \pm 283$
\end{tabular}

Table 12.4: Elasticity values found by numerical simulations from supine-to-prone $\left(E_{s p}\right)$ and prone-to-supine $\left(E_{p s}\right)$ in four different resolution scales and then averaged, using SOFA.

\begin{tabular}{c|c|c|c} 
phantom & $E_{s p}$ & $E_{p s}$ & Mean $E$ \\
\hline I & $5046 \pm 272$ & $5252 \pm 307$ & $5145 \pm 254$ \\
II & $4290 \pm 351$ & $4298 \pm 273$ & $4291 \pm 276$ \\
III & $5291.52 \pm 383.00$ & $5639 \pm 456$ & $5459 \pm 400$ \\
IV & $7916 \pm 1165$ & $7564 \pm 957$ & $7731 \pm 1016$
\end{tabular}

Table 12.5: Elasticity values found by simulating from supine-to-prone $\left(E_{s p}\right)$ and prone-tosupine $\left(E_{p s}\right)$ in four different resolution scales and then averaged, using FEBio as software package.

\subsubsection{Numerical simulation by supine-prone and prone- supine matching in SOFA}

Table 12.4 lists the elasticities obtained by numerical simulation from supine to prone position and vice-versa, in SOFA. As opposed to the $\beta$ computation method, the prone-supine simulation method also takes nonlinearities into account which theoretically results in a more accurate estimate of the $E$ value.

For each resolution, up to ten simulation runs are needed to find the final $E$ value in which the error vanishes. This makes the method relatively slow, requiring about twenty minutes of computation time on a quad-core $2.5 \mathrm{GHz}$ computer per phantom. By parallelizing computations of the four phantoms, the total computation time for all $E$ values was measured to be approximately half an hour.

\subsubsection{Numerical simulation by supine-prone and prone- supine matching in FEBio}

Table 12.5 lists the elasticity values using the FEBio software package. The resulting elasticity values are comparable to those obtained by SOFA. A relatively high variance is present in Phantom IV, which may be caused by side effects in the software package. 


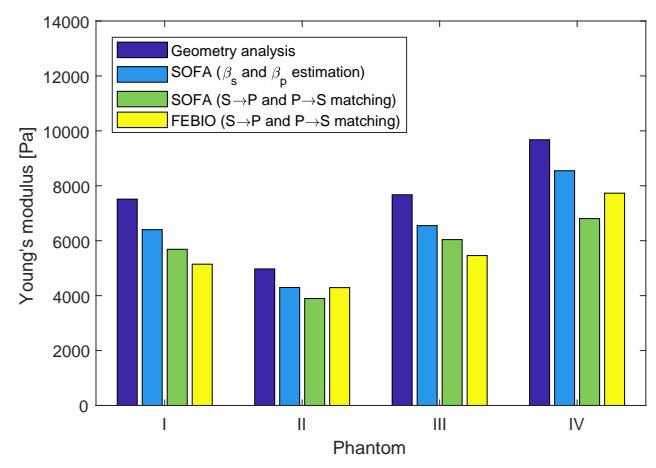

Figure 12.8: Young's modulus for four phantoms, derived by four different methods.

\begin{tabular}{c|c} 
phantom & mean $E$ \\
\hline I & $6188 \pm 886$ \\
II & $4364 \pm 387$ \\
III & $6430 \pm 815$ \\
IV & $8190 \pm 1057$
\end{tabular}

Table 12.6: Mean elasticity values for each phantom, taken as the average of the seperate values derived by the four different methods.

\subsubsection{Comparison of different elasticity measurement meth- ods}

Figure 12.8 graphically shows the elasticity of the four phantoms, derived using the different methods, while Table 12.6 lists the overall phantom elasticities, averaged from the four different methods.

Two methods using SOFA were presented: the first one numerically simulates the $\beta_{s}$ and $\beta_{p}$ values from the supine and prone meshes seperately and measures the tip displacement $D$, from which the phantom's elasticity $E$ is derived. The second method involves finding $E$ directly by simulation from supine to prone position such that the tip position error is eliminated. The first method seems to give consistently higher estimates for $E$, especially for phantom IV. Possible causes might be the nonlinearity of the displacementto- $g / E$ ratio, i.e. $\beta$ cannot be considered constant for the required range of displacements. Furthermore, the deformations of the tip resulting from proper FEM simulations influence the displacement calculations. As the second algorithm uses the iterative point cloud algorithm to minimize tip displacements and also takes nonlinear effects into account, that one can be considered more accurate than the first one.

The numerical results from FEBio simulations are in accordance with SOFA 
Ch 12: Analytical derivation of elasticity in breast phantoms for deformation

matching simulations, which is an indication that the simulations are consistent.

\subsection{Discussion}

We have presented a new method to analytically evaluate the elasticity of breast phantoms, from a pair of MRI scans in prone and supine position. The values found from analyzing the gravity-induced deformations are comparable to the elasticities derived from FEM simulations using FEBio and SOFA, with deviations of up to $18 \%$. A study on nine geometric shapes has shown that the method is not only applicable to breast shapes, but also to other bodies and geometric objects as long as it is substantially supported by a planar rigid base.

The advantages of the analytical method are that the elasticity calculation is very fast $(<1 \mathrm{~s})$ and takes each individually scanned voxel into account, without need for mesh generation. As the voxel intensity in a scan gives certain information about tissue type, density and/or elasticity (depending on scanning protocol), tissue inhomogeneities can be directly incorporated in the analytical computations. The main limitations are that the method is only suitable for deformations in the linear range, and that the shapes must be substantially supported by a planar base perpendicular to the gravitational field.

The fact that a human breast is relatively flexible and the chest wall is not planar but cylindrically shaped, makes clinical application difficult. An artificial planar support base could be constructed by using a patient-mounted breast coil, ideally in combination with a patient rotation system. The presented methods may also have applications in different domains, wherever deformation of bodies are involved in situations that meet the aformentioned boundary conditions The conclusion is that under specific conditions, the elasticity of a deformable object such as a human breast can be quickly computed from a pair of volumetric scans with sufficient accuracy, without need for FEM simulations. This promising result opens the door to new applications which can benefit from this complementary and near-realtime elasticity computation method. 


\section{CHAPTER 13}

\section{D Ultrasound Elastography Reconstruction Using Acoustically Transparent Pressure Sensor on Robotic ARM}

V. Groenhuis, A. Nikolaev, S.H.G. Nies, M.K. Welleweerd, L. de Jong, H.H.G. Hansen, F.J. Siepel, C.L. de Korte, S. Stramigioli. 3D Ultrasound Elastography Reconstruction Using Acoustically Transparent Pressure Sensor on Robotic Arm. Submitted to The 11th International Conference on Information Processing in Computer-Assisted Interventions (IPCAI), Munich, 2020 

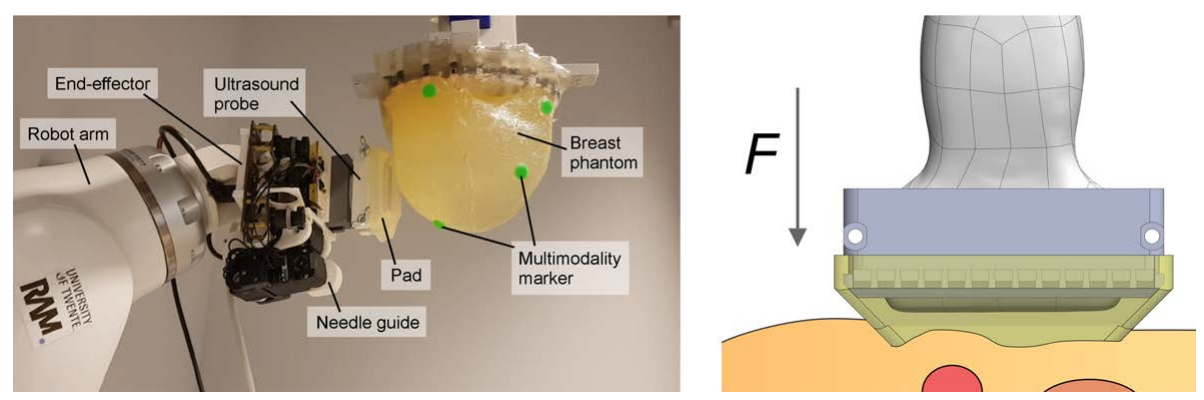

Figure 13.1: Left: Experimental setup. The ultrasound probe with pad is mounted on the robot arm's end-effector, the breast phantom hangs in prone position. Right: Working principle of the pressure sensor. The observed pad thickness is related to the local pressure by the known Young's modulus of the pad.

\subsection{Purpose}

In breast cancer screening, certain suspicious lesions are only detectable on MRI and not by mammography (x-ray) or US (ultrasound). A biopsy sample of the lesion is required for accurate pathology assessment. The manual MRI-guided biopsy procedure is inaccurate and inefficient: breast tissue may deform due to body movements, muscle contractions, and needle-tissue interactions. These changes are not visible due to the low sampling frequency. This has led to the launch of research projects focused on the introduction of surgical robotics to overcome the existing limitations and improve the accuracy of biopsies of MRI-visible lesions [54].

The MURAB (MRI and Ultrasound Robotic Assisted Biopsy) project aims to solve these challenges by coregistering MRI images to 3-D US data and 3-D elastography maps acquired using a robotic arm. These are then fused into one map by using multimodality markers on the skin. Based on these scans, a patient-specific finite-element model is constructed to simulate the deformations resulting from probe-tissue and needle-tissue interactions. This allows optimization of the needle insertion path planning task. The radiologist then inserts the needle in a robotically-steered needle guide towards the target. Tissue movements are tracked in real-time using the ultrasound probe, allowing to adapt the insertion trajectory if needed.

This paper focuses on the acquisition of 3-D elastography on phantoms with a novel acoustically-transparent pressure pad and strain imaging. Several 2-D planes are measured after which a $3-\mathrm{D}$ model can be reconstructed. 


\subsection{Methods}

Elastography data is reconstructed from 2-D pressure and displacement maps. Both are simultaneously acquired using an ultrasound probe (L10-5v, Siemens P500 system) equipped with an acoustically transparent pressure pad. The pad is made of PVC Plastisol attached to a rigid frame mounted on the US probe. A schematic drawing of the pad is shown in Fig. 13.1 (right). The combination of the pad and the robotic arm is an improvement on the strain elastography, which uses manual compression[73]. The compression is done robotically instead of manually, which makes the movement more consistent and ensures the orientation with respect to the tissue is always perpendicular. Since the mechanical properties of the pad are known, the exerted force can be derived based on the deformation of the pad.

A heterogeneous breast phantom was manufactured using PVC Plastisol mixed with plasticizer and silica gel. The skin has a higher stiffness than fibroglandular and fatty tissue. A rigid frame mimicks the rib cage, allowing to position it in prone position. The probe is placed orthogonally on its breast surface by the robot arm as shown in Fig. 13.1. A gradually increasing force is exerted on the breast while the probe is constrained to move in image depth direction only. The maximum force is tuned to the stiffness of the specific breast or phantom, in this case $15 \mathrm{~N}$. This results in the compression of tissue in the US imaging plane, locally displacing the skin by up to two centimetres. The first scan is the reference image of the sequence, in which the probe just makes contact with the surface and the stress and strain are approximately zero. A small bias in stress or strain is acceptable as relative changes are used in elasticity calculation. The subsequent frames are used to calculate incremental tissue displacements using a cross-correlation-based block matching algorithm and converted to a strain map.

In order to derive a quantitative measure of elasticity in the imaging plane, the stress must also be known. The Young's modulus is defined as the ratio between stress and strain [66]. For this, an acoustically transparent pad is placed on the US probe. This pad has a known thickness and material stiffness. The skin-pad boundary is estimated by edge detection of the US scans. By measuring the local thickness in each scan and comparing it to the reference scan thickness, the local pad strain is estimated. As the Young's modulus of the pad is known, the stress distribution inside the pad can be derived. This stress distribution over the surface of the pad is propagated to the US scan region. The exact stress distribution depends on several factors including the breast shape, amount of deformation and material properties. Based on FEM (finite-element model) simulations and earlier experiments a linear attenuation coefficient with horizontal smoothing factor is estimated. From the stress and strain maps a quantitative measure of the elasticity is calculated in every 

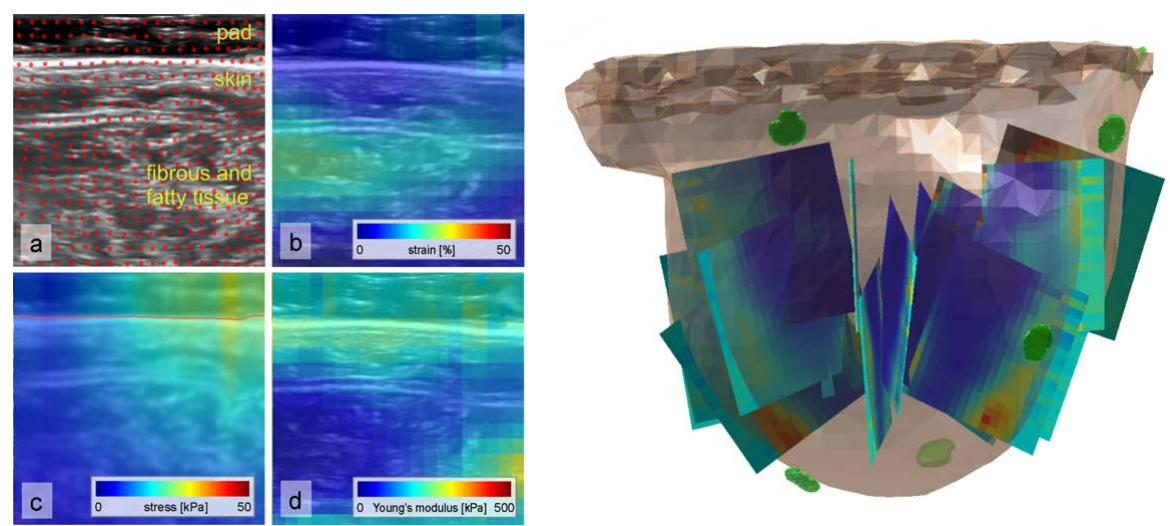

Figure 13.2: Left: Post-processing of US data. a) Displacement tracking by block matching algorithm. b) Strain map showing amount of compression. c) Pad boundary and pressure distribution. d) Young's modulus as calculated from stress and strain maps. Right: 3-D rendering of the phantom with sixteen elasticity measurement slices.

frame, represented in the reference frame. Finally, the output elasticity map is calculated by taking the median elasticity in each voxel, removing the outliers. This process is repeated for all sixteen sites on the breast and visualized in a $3-\mathrm{D}$ rendering.

\subsection{Results}

The post-processing graphs of a sample frame of one site are shown in 13.2 (left). The pad is mostly anechoic, the skin is hyperechoic and deep tissue includes different levels of echogenicity. In Fig. 13.2a, the local displacement of tissue in this frame relative to the reference frame is visualized using a lattice grid. In Fig. 13.2b, the strain is calculated from the compression in vertical direction. In Fig. 13.2c, the pad-skin boundary is shown as a red line, measured to be $8.8 \mathrm{~mm}$ on average in the reference frame and $8.0 \mathrm{~mm}$ in the sample frame. The local change in thickness is used to estimate the pressure in the pad and the rest of the frame. In Fig. 13.2d, the stress and strain maps are combined to calculate the elasticity of the sample frame. It can be observed that the skin has a higher Young's modulus (approx. $250 \mathrm{kPa}$ ) than most of the deep tissue (up to $150 \mathrm{kPa}$ ) which is consistent with the fabrication process of the phantom.

Fig. 13.2 shows a $3-\mathrm{D}$ rendering of the breast phantom as scanned in MRI and registered using the eight multimodality markers. The square-shaped colored surfaces represent the elasticity measurement results at the sixteen sites on the phantom. It is observed that the stiffness of skin and deep tissue are 
consistent across the different slices, and that some noise is present in specific areas of the elasticity maps at certain sites.

\subsection{Conclusion}

The MURAB project shows that it is possible to quantitatively acquire elastography data using a ultrasound probe equipped with an acoustically transparent pad. Measurements on a PVC phantom show consistent results across different sites of the phantom.

The elastography acquisition method scans one plane at a time, with every plane taking five seconds. While it is not possible to perform elastography scanning in one sweeping motion over the surface, it may be possible to speed up acquisition by recording less frames in each plane to speed up acquisition and/or acquire elastography data from more slices.

Estimating the stress map inside tissue is a difficult point. Additional exvivo and/or in-vivo experiments should be conducted to gather more reference data for calibration, and to assess the accuracy of the described method in comparison to other techniques. 

Real-time Deformation Tracking System for BREAST Biopsy

V. Groenhuis, F.J. Siepel, S. Stramigioli. Real-time Deformation Tracking System for Breast Biopsy. Submitted to The 11th International Conference on Information Processing in Computer-Assisted Interventions (IPCAI), Munich, 2020 

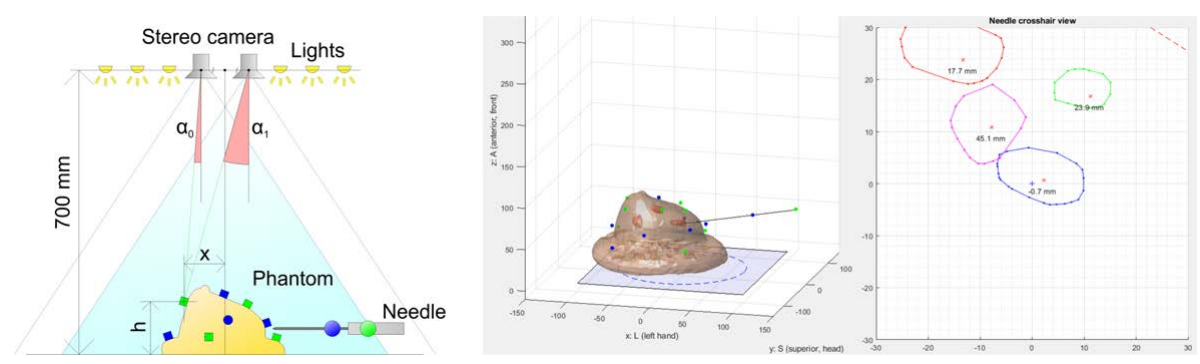

Figure 14.1: Left: Deformation tracking setup. The stereo camera detects and tracks colored markers on the breast phantom and needle within its common field of view. Center: 3D rendering of phantom, lesions and needle. Right: Lateral position and coaxial distance of lesions relative to needle, with the blue lesion in the center being in the needle path.

\subsection{Purpose}

In breast cancer screening, suspicious lesions may be found that need to be checked for malignancy. A biopsy procedure is used for accurate histological confirmation, usually under ultrasound guidance. If the lesion is only visible on MRI, a MRI-guided biopsy procedure is necessary. Deformations of the breast make it difficult to bring the needle precisely towards the selected target: the current manual MRI-guided biopsy procedure is inaccurate and would benefit from a technique that allows real-time tracking and visualization of the lesion during needle insertion [29].

The goal of the MRI and Ultrasound Robotic Assisted Biopsy (MURAB) project is to solve the aforementioned challenges involved in targeting MRIvisible lesions, by combining deformation modeling and tracking techniques with a robotically steered biopsy system. This paper focuses on the sub-task of estimating present lesion locations by optically tracking 17 colored markers on the skin. By visualizing the estimated lesion locations in real-time $(9 \mathrm{~Hz})$, this approach allows precise insertion of a biopsy needle towards the chosen target under the presence of deformations.

\subsection{Methods}

A soft PVC breast phantom (Fig. 14.2) was constructed with four embedded lesions and 17 multimodality-visible fiducials (blue and green, $10 \mathrm{~mm}$ diameter) filled with vaseline attached to the skin. Two more markers (20 mm diameter) are attached to the needle. The phantom with markers was previously scanned in a $0.25 \mathrm{~T}$ (G-Scan Brio, Esaote SpA, Genoa, Italy) MRI scanner and subsequently segmented by intensity thresholding from which the reference MRI model was constructed. The stereo vision system consists of two synchronized 

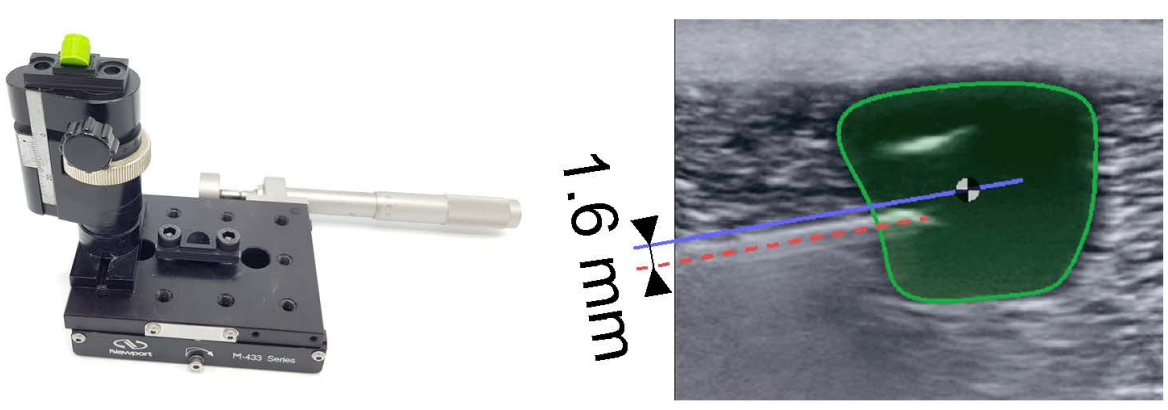

Figure 14.2: Left: Setup for manipulation and tracking of one marker in $\mathrm{X}$ and $\mathrm{Z}$ directions. Right: Example ultrasound confirmation scan of lesion (green) targeted by needle (red).

cameras (Kayeton Technology Co. Ltd, Shenzhen, China), with resolution 1280x960 pixels, $3.75 \mathrm{~mm}$ pixel size and $8 \mathrm{~mm}$ focal length. The baseline (distance between lenses) is $60 \mathrm{~mm}$ and the camera is positioned $700 \mathrm{~mm}$ above the table, alongside with a series of lights as shown schematically in 14.1.

During the actual procedure, the stereo vision system segments markers by thresholding in the (G, B-G, R-G) colorspace, which is as effective as HSV or $\mathrm{YCbCr}$ but significantly faster in conversion from RGB. The resulting blob centroids are stereo matched and triangulated, forming a sparse 3D point cloud. The coordinates of the markers with observed diameter around $10 \mathrm{~mm}$ are registered to that of the MRI phantom model using a combination of three different algorithms: by finding markers attached to base, by building feature descriptors from inter-marker distances, and/or by using prior knowledge from the previous frame. From the registered marker pairs, a non rigid transformation is calculated based on thin-plate splines (TPS), which is an interpolation function that minimizes the total bending energy[98]. The lesions transformed this way are visualized on the user interface together with the projected needle path as calculated from the two larger markers (Fig. 14.1 (center)). All computer vision processing and visualizations are performed in MATLAB, with an update rate of $9 \mathrm{~Hz}$.

The system was evaluated on two levels. Firstly, the tracking accuracy and precision of a single marker were measured using the setup in Fig. 4 (left), in 2D image coordinates and in reconstructed 3D world coordinates. Secondly, each of the four lesions in the phantom was manually targeted three times from different angles, guided by the user interface. The phantom and needle were positioned and oriented such that as many markers as possible are detectable by the stereo camera without overlapping each other. After insertion, the resulting targeting error distance (lateral to the needle) was measured using ultrasound (Fig. 4, right). 


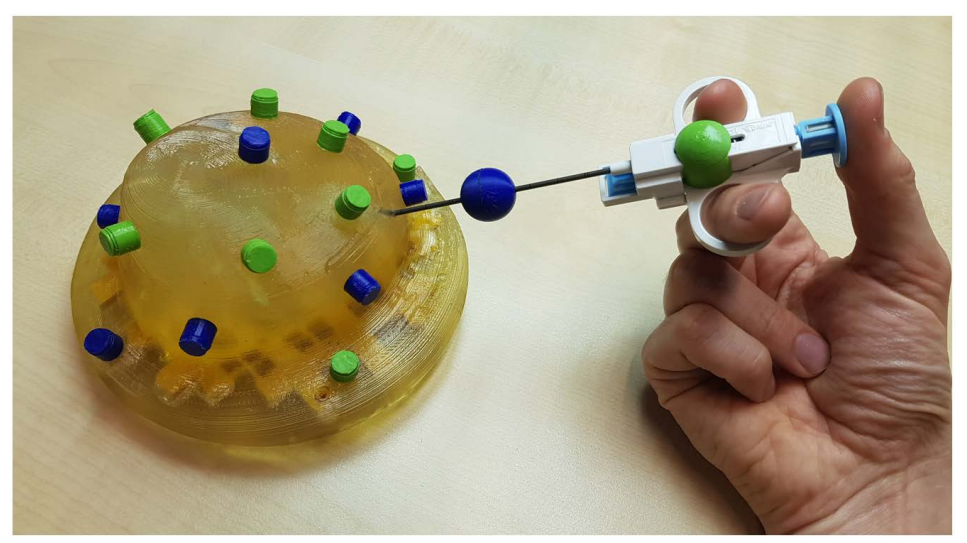

Figure 14.3: Left: Setup for manipulation and tracking of one marker in $\mathrm{X}$ and $\mathrm{Z}$ directions. Right: Example ultrasound confirmation scan of lesion (green) targeted by needle (red).

\subsection{Results}

When tracking one marker in image coordinates, the standard deviations $\sigma_{x}$ and $\sigma_{y}$ were measured to be between 0.06 and 0.11 pixels, depending on the marker location within the region of interest. After triangulation in world coordinates, the standard deviations $\sigma_{x}$ and $\sigma_{y}$ were measured to be between 0.02 and $0.06 \mathrm{~mm}$, and $\sigma_{z}$ between 0.3 and $1.1 \mathrm{~mm}$.

In the needle insertion experiment, twelve sites were targeted (Fig. 14.3). For each site, the needle was aimed slightly above the projected lesion to compensate for the downwards-oriented beveled tip of the needle. In one case, the lateral error distance could not be evaluated as feasible ultrasound probe locations were obstructed by markers on the skin, so this specific case was repeated by using an alternative insertion approach. From the twelve successful measurements, the lateral targeting error was found to be $(4.5 \pm 1.6) \mathrm{mm}$ (range $2.1 \mathrm{~mm} . .7 .0 \mathrm{~mm})$.

\subsection{Conclusion}

The precision of marker tracking in world coordinates is excellent in $\mathrm{x}$ - and y-directions $(0.1 \mathrm{~mm})$, but relatively poor in $\mathrm{z}$-direction $(1 \mathrm{~mm})$. This could be improved by e.g. increasing the stereo baseline (at the cost of reduced observability of markers on the side of the phantom), or by increasing the number of cameras[17]. In practice, the relatively high update rate of $9 \mathrm{~Hz}$ already allows to smoothen out spatial measurement noise without significantly compromising on temporal accuracy. 
The geometric, non-rigid transformation method based on registered skin markers has shown to be effective in estimating lesion positions for this phantom, under the presence of deformations. The resulting mean targeting error of $4.5 \mathrm{~mm}$ is on par with state-of-art breast tumor tracking techniques[10]. An important limitation is that the current implementation ignores physical properties such as tissue incompressibility or heterogeneous properties of tissue. With biomechanical modeling being a key part of the MURAB project, the next step is to combine realistic deformation simulations with optical skin tracking, and also use a robotic arm to automatically align the needle with the target lesion. With these techniques incorporated, it is expected that the targeting error can be further reduced, eventually leading to measurable improvement of breast cancer screening of MRI-visible lesions in clinical practice. 



\section{DeFormation COMPENSATION IN}

\section{RoboticalLy-Assisted BREASt Biopsy}

V. Groenhuis, E. Tagliabue, M.K. Welleweerd, F.J. Siepel, J.D. Munoz Osorio, B.M. Maris, F. Allmendinger, D. Dall'Alba, U. Zimmermann, P. Fiorini, S. Stramigioli. Deformation Compensation in Robotically-Assisted Breast Biopsy. Submitted to The 11th International Conference on Information Processing in Computer-Assisted Interventions (IPCAI), Munich, 2020 


\subsection{Purpose}

A major challenge of current breast biopsy procedures is lesion displacement due to needle-tissue interaction, respiration and involuntary motions, possibly causing the needle to miss the target. These deformations are intrinsically accounted for when the procedure is performed under ultrasound (US) guidance, but the low US resolution makes target visualization often impossible. By contrast, MRI-guided biopsies provide high-resolution images with excellent sensitivity, but they do not account in any ways for breast deformations. The MRI and Ultrasound Robotic Assisted Biopsy (MURAB) project aims to solve this challenge by the use of a combination of technologies.
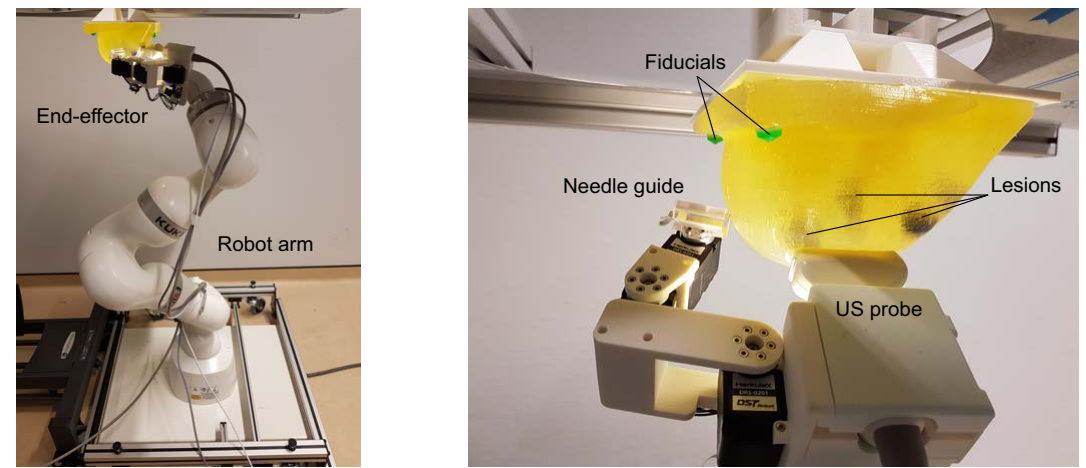

Figure 15.1: Experimental setup showing phantom (with fiducials and lesions) and robot arm with end-effector consisting of ultrasound probe, stereo camera and steerable needle holder.

A robotic arm (Fig. 15.1, left) outside the MRI scanner is equipped with a US probe, stereo camera with lights and a steerable needle guide (Fig. 15.1, right). The envisaged workflow is that the pre-operative MRI scan is coregistered with a robotically-acquired 3-D ultrasound model after which the biopsy intervention is planned taking tissue deformations induced by probetissue contacts into account. During the intervention, real-time 2-D ultrasound tracking is employed to perform further trajectory corrections.

The purpose of this study is to assess the feasibility of predicting lesion displacements due to probe-tissue interactions, by finite-element (FE) simulations. This is evaluated by performing a series of biopsies on a phantom under continuous contact with an ultrasound probe.

\subsection{Methods}

A soft PVC (polyvinyl chloride) plastisol phantom was created containing two stiff ink-stained lesions sized $11 \mathrm{~mm}$ situated $2 \mathrm{~cm} . .3 \mathrm{~cm}$ beneath the stiff skin 
1. Target lesion definition

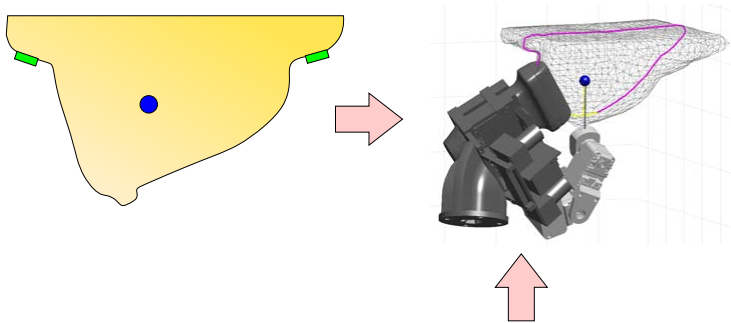

4. Update lesion coordinates

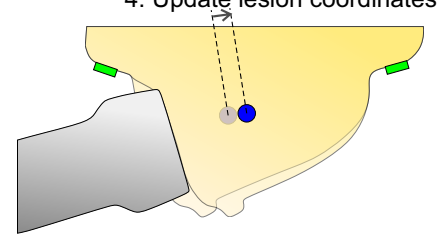

2. Intervention (re-)planning

3. FE simulation (SOFA)

Figure 15.2: Workflow in breast biopsy with deformation compensation. The target lesion is selected (1) and intervention planned (2). The intervention is simulated (3), shifting the lesion to a new location. The intervention is re-planned using this new lesion location and re-simulated using the original lesion location. Once the lesion's new location is stable the biopsy is executed (5).

surface [130]. Five green-colored PVC plastisol markers are attached to the surface, separated by rigid spacers with thickness $1.5 \mathrm{~mm}$ to enable automatic marker segmentation of MRI scans. The phantom has an estimated average stiffness of $4000 \mathrm{~Pa}$ and is mounted on a frame above the robot arm (Fig. 15.1).

The MRI-scanned and segmented phantom is registered to the robot coordinate frame using rigid registration of the five markers by computer vision. One of the lesions is chosen as target and the intervention procedure planned, which involves choosing a location of the ultrasound probe and the needle insertion point such that the needle trajectory is within the ultrasound plane. The probe indents the skin surface by $1 \mathrm{~cm} . .2 \mathrm{~cm}$ to ensure an optimal acoustic coupling, which is fundamental to be able to follow the needle on US images during the insertion.

Breast deformations induced by US probe pressure are simulated via FE method using the SOFA framework ${ }^{1}$. Probe-tissue interaction is modelled as a frictionless contact problem where interaction forces are computed each time as those able to satisfy the impenetrability constraint [34]. Similarly to [130], the breast is discretized with linear tetrahedra and described as a homogeneous Neo-Hookean material.

Based on the FE simulation the intervention procedure is re-planned with

\footnotetext{
${ }^{1}$ www.sofa-framework.org
} 


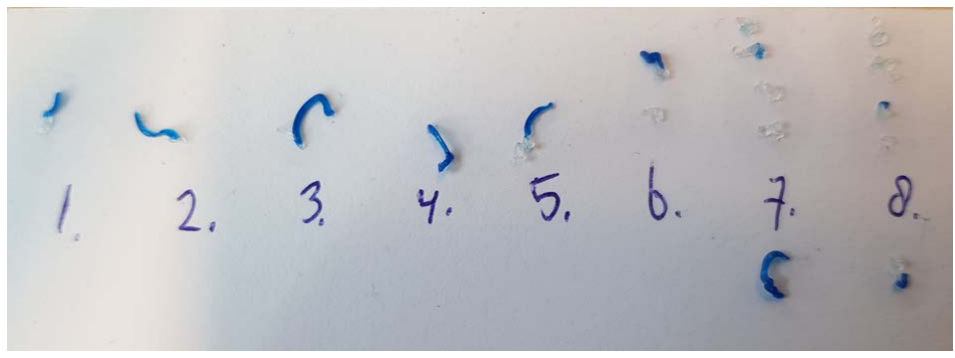

Figure 15.3: Samples acquired in eight biopsies. The first five were a direct hit, the last three required two or more attempts.

the updated lesion location (obtained by interpolation using FE shape functions) such that the deformation is taken into account in the new planning. This process is repeated until the simulated lesion displacement has stabilized. Figure 15.2 graphically summarizes the workflow.

The intervention procedure is then executed, steering the ultrasound probe and needle guide robotically to its position and inserting the biopsy needle manually through the guide to the pre-determined depth. The biopsy gun is then fired and the sample taken out. The sample is manually inspected to evaluate the extent to which it contains ink-stained matter. A biopsy is classified as a hit if the ink-stained portion of the sample is at least $2 \mathrm{~mm}$ in size. This process is repeated for a total of eight distinct interventions (four per lesion).

\subsection{Results}

Fig. 15.3 shows the samples acquired in all biopsies. The first five biopsies all resulted in a direct hit, while the last three biopsies were missed on the first attempt.

Three of eight biopsies, were a miss on the first attempt, indicating needle positioning errors in excess of $5.5 \mathrm{~mm}$ (half the diameter of the lesion). There are different possible reasons for this inaccuracy:

- The needle holder is driven by geared servo motors which are not free of backlash. The parasitic motions in the motors allow the needle to tilt over several degrees, resulting in a shifted insertion trajectory and a higher chance of missing the lesion.

- In the planning phase, we are assuming the 18 -gauge $(1.27 \mathrm{~mm})$ needle to be rigid, following a straight path without deforming tissue. This assumption may not hold in real scenarios, where needles (especially with 
a beveled tip) can actually bend during insertion and also cause additional deformations.

- Modelling breast-probe interaction as a frictionless problem allows probe sliding motion in some cases. However, in our setup, the contact surface remains constant. Accounting for frictional effects might help to reflect the actual behavior.

- Registration errors in visual marker detection and registration also contribute to targeting errors. In rigid registration of the five fiducials the mean error was measured to be approximately $2 \mathrm{~mm}$.

\subsection{Conclusion}

The study has shown that it is possible to effectively compensate for deformations using FE simulations within the MURAB workflow. Not all biopsies were a direct hit, indicating errors in excess of $5.5 \mathrm{~mm}$. Several aspects such as component calibration, needle guide actuation, needle depth guiding system, FE simulation accuracy and phantom registration need to be investigated and improved where possible, in order to obtain an acceptable success rate in the breast biopsy procedure. With these factors taken into account, MURAB may be a promising system for performing breast biopsies in the future. 



\section{Part V \\ General Discussion and Conclusion}





\section{CHAPTER 16}

\section{DisCUSSION}

Two different robotic solutions were presented to solve the clinical challenge of the biopsy procedure for MRI-visible lesions in the breast: the MURAB robotic system outside the MRI scanner and the Stormram/Sunram biopsy robots inside the MRI scanner. Besides, a non-robotic, single-camera setup was also demonstrated. All three solutions demonstrate the possibility of inserting a needle towards a given target under guidance of different imaging modalities, and perform a biopsy. The overall research covered multiple topics, which are discussed in this chapter.

\subsection{Scientific and technological discussions}

This section discusses several scientific and technical aspects of the robotic systems including subcomponents developed during the research.

\subsubsection{Breast phantoms}

Phantoms can be constructed from PVC plastisol to mimick real breasts. Chapter 12 describes techniques to make heterogeneous phantoms with a relatively stiff skin and soft inner tissue with lesions, using a set of moulds. The phantom design can be based on the MRI scan of a human breast to mimick the exterior shape and internal structures. Semi-random spaghetti-like structures can be added by adding it in the soft tissue before solidification, and tissue types can be specifically made visible on ultrasound by the addition of silica gel in 
various concentrations. Structures can also be made optically visible by using a colorant.

One important drawback of PVC plastisol phantoms developed during this research is that these are on the stiffer side of the spectrum. The difficulty in fabricating phantoms sufficiently mimicking the physical properties of relatively soft breasts is that PVC plastisol tends to disintegrate under stress if it is too soft. Further research involving new combinations of materials and techniques is needed in order to develop phantoms with more realistic properties.

\subsubsection{Pneumatic stepper motors}

Chapters 2, 3, 4 and 5 show that rapid prototyped pneumatic stepper motors are able to actuate a linear, curved or rotational axis in well-defined steps with significant output force or torque. Several design parameters and manufacturing techniques are available. The specific choice of design parameters depends on the target application, such as the actuation of a specific MR safe robotic system.

The most difficult part in the construction of a stepper motor is the moving seal. This seal has to be able to maintain pressure in the associated pressurized air chamber while sliding along the $3-\mathrm{D}$ printed cylinder walls without providing excessive friction. In the scale of MR safe robotics such a seal is best laser-cut from a silicone rubber sheet in which the seal dimensions are carefully tuned for the specific stepper motor. Seals for large-scale stepper motors can be cut manually using a cutting guide. It is almost impossible to make the seal completely airtight due to the specific cross-sectional shape and 3-D printing characteristics, but small leakages are acceptable if the pressure differential across the seal can be maintained.

A limitation in pneumatic stepper motors is the low effective bandwidth in an MRI environment due to the long pneumatic tubes running from the control room to the robot. This limitation can be circumvented by the use of dual-speed stepper motors which can execute both large and small steps on the same axis (Chapter 4).

\subsubsection{MR safe robotics}

Five iterations have been developed within the Stormram/Sunram research line as described in Chapters 2, 6, 7, 8, 9 and 10. Every iteration is a significant improvement over its predecessor with the latest iteration (Sunram 5) demonstrating new capabilities thanks to its dual-speed motor technology and other advanced features. Still, the technology readiness level (TRL) is estimated to be no higher than 3 of 4 . It is a proof of concept which needs significant further development in order to reach the level suitable for clinical practice. Several critical components are missing: an intuitive user interface 
coupled to MRI data, sterilization techniques, robust calibration methods, additional safety measures, integration into a real breast coil, compatibility with off-the-shelf MRI needles, etcetera. If all these challenges can be solved then the technology has the potential to supplement, or even replace, the current practice in MRI-guided breast biopsy.

\section{Kinematic configuration}

Stormram 1, 2 and 3 use a clear parallel kinematic chain while Stormram 4 and Sunram 5 use a predominantly serial kinematic chain. In theory a parallel kinematic chain offers higher stiffness (and accuracy) at the cost of a smaller workspace. Stormram 4 has been designed to provide a sizeable workspace without compromising on stiffness or accuracy. This has been made possible due to the direct integration of each stepper motor on its actuated (linear or curved) rack, eliminating any backlash commonly found in traditional gearboxes and rack-pinion systems. Furthermore, the two revolute joints actuated by curved stepper motors are constructed in such a way that a truss-like structure is created, further improving stiffness.

Sunram 5 uses dual-speed motors on multiple axes plus a needle firing mechanism, resulting in a kinematic chain with more serial links (seven in total) than in the Stormram 4 (four serial links). Still, the overall system appears to be robust enough to position a needle with millimeter accuracy in free air. One specific actuator for inserting the needle shows a slightly wobbly motion, but this could be eliminated by incorporating an additional linear guide on the associated axis.

\subsubsection{MURAB}

The MURAB setup includes an off-the-shelf robotic arm with end-effector. As described in Chapter 11 this includes an ultrasound probe, a stereo camera and a robotic needle guide. It has been shown that it is possible to construct patient-specific models from MRI scans, perform marker-based MRI-world registration, generate contact scanning trajectories, acquire 3-D ultrasound data, perform elastography scanning, simulate deformations induced by probe-tissue interactions, measure deformations in real-time and perform biopsy of MRIvisible lesions.

\section{Needle guide}

The needle guide on the end-effector is driven by three off-the-shelf smart servo motors placed in a serial kinematic chain. The construction of these specific motors involve a spur gear transmission with a small, but noticeable amount of backlash. Especially the backlash in the first joint results in reduced accuracy 
in the positioning of the needle guide. This could be remedied by incorporating backlash-free servo motors such as one containing a strain wave gear transmission. Nevertheless, the needle insertion accuracy in free air, relative to the ultrasound probe, was measured to be in the order of one millimeter.

\section{Multi-modality markers}

Markers placed on the surface of the breast are needed for MRI-robot registration and in deformation tracking in certain experiments. The challenge is to fabricate markers which are well visible (and automatically segmentable) in MRI, are detectable by computer vision, and are compatible with robotic ultrasound scanning of the breast. This turned out to be difficult and it has not yet been fully solved in this research. Markers may be wiped off the skin surface by the US probe if adhesion is suboptimal, especially in rigid markers with unfavourable shapes. In case of rigid MRI-robot registration the US compatibility requirement can be relaxed by putting four markers near the chest of the patient which cannot be reached by the probe, plus one at the tip of the breast. This way 3-D US scanning is possible without touching the markers at all. In case of real-time tracking of deformations using computer vision (Chapter 14) many more markers are needed which is incompatible with the 3-D US scanning procedure. Possible solutions which could be explored in future research is to develop soft, thin adhesive markers filled with liquid, or markers consisting of multiple segments in which the relatively large, MRI detectable part can be individually detached if needed.

Multi-modality markers can be fabricated as a cylinder-shaped piece of colored PVC plastisol mounted on a disc of hard plastic. An alternative is to use 3 -D printed capsules filled with vaseline. In this research the computer vision systems utilized specific colors within the visible spectrum such as green or blue. The drawback is that the observed hue and luminance components of the markers as perceived by the computer vision system are dependent on the lighting situation. A possibly more robust choice would be to incorporate infrared-reflective surfaces on the markers to make these detectable on both MRI and infrared-based marker tracking systems.

\section{Deformation simulation and compensation}

Chapter 15 shows that it is possible to simulate deformations due to probetissue interactions. This is a suitable method to estimate the updated location of the lesion after the ultrasound probe is positioned. The simulation uses a FEM model which is a simplified representation of the breast. For example, current models consider homogeneous models only while a real breast has structures of varying stiffness which affect the way it deforms. For precise biopsies some form of imaging feedback should be used in conjunction with simulations, 
in order to measure the actual deformations and correct the needle insertion trajectory accordingly.

\section{Real-time deformation tracking}

The surface of the breast can be tracked in real-time using a fixed stereo camera positioned at some distance from the breast, such that all markers on the surface are within view of the computer vision system. In Chapter 14 it has been demonstrated that these markers can be registered to the corresponding markers in the MRI model in real-time, even if a minority of the markers are obstructed from view, and that an elastic transformation of the segmented MRI model can be projected over the actual breast. This allows to estimate the location of the lesion based on the skin deformation, enabling a closed-loop biopsy procedure in which the radiologist inserts the needle manually while the system provides guidance on correct needle angulation and displacement. The targeting accuracy was measured to be approximately $4.5 \mathrm{~mm}$ and this could be improved by using multiple camera units employing an infrared-based marker tracking strategy, and/or by incorporating a FEM simulation model rather than geometric interpolation for projecting the location of the lesion.

\section{Elastography using acoustically transparent pressure pad}

The 3-D elastography procedure described in Chapter 13 involves positioning a deformable pad with known stiffness over the ultrasound probe. This specific technique is relatively new and experiments on a breast phantom have shown that it is possible to measure the elasticity quantitatively. In combination with a robot arm a 3-D ultrasound map of the phantom can be constructed, although the resulting accuracy is dependent on several factors: the knowledge of the physical properties of the pad, the correctness of the pressure distribution estimation inside the phantom and the quality of the ultrasound speckle tracking algorithm. All these aspects need further research and comparison studies before valid elastography observations can be made. The procedure also takes a relatively long time, in the order of a few minutes depending on the scanned volume, but if it results in accurate $3-\mathrm{D}$ elasticity maps then it would allow for more realistic FEM simulations and eventually more precise biopsies in workflows that make use these simulations.

\subsection{Accuracy of biopsy needle placement}

Three workflows have been developed to target MRI-visible lesions inside the breast. Each workflow uses its own combination of technologies. The critical evaluation aspect is the needle insertion accuracy which is described in this section. 


\subsubsection{Stormram/Sunram}

The Stormram 4 has been evaluated in an MRI scanner in Chapter 9 and it was found that the Stormram 4 has an absolute needle positioning accuracy of $1.3 \mathrm{~mm}$. This method does not take tissue displacements into account, so the actual error in targeting lesions may be higher on average unless real-time trajectory corrections are incorporated in the workflow. Still, it provides a strong base demonstrating the capabilities of the Stormram/Sunram line of robotics. When coupled with a suitable breast fixation system, such as the one designed for the Sunram 5, it is possible to perform quantitative measurements of the accuracy in biopsying lesions within breast phantoms.

If this procedure would be applied to human patients then the major challenge is how to perform needle insertion safely with the patient being inside the MRI scanner. The idea of a robotic system performing an invasive action in a claustrophobic situation needs unconditional trust in functioning of the robotic system, and well-thought procedures that describe every possible aspect of the workflow. Sophisticated designs of the robot and controller are required to ensure safety in all phases of the procedure.

\subsubsection{MURAB with deformation compensation}

The needle insertion method described in Chapter 15 uses the MURAB setup. This specific experiment involved eight biopsies of $11 \mathrm{~mm}$-sized lesions inside a breast phantom with probe-tissue induced deformations in the range of 1-2 cm (at the probe-breast contact point). Of these eight biopsies five were a direct hit, indicating an average targeting error in the order of $5 \mathrm{~mm}$. It later turned out that the needle guide mechanism was not properly calibrated, indicating potential for better results in follow-up experiments and that smaller-sized lesions can also be targeted.

\subsubsection{Real-time deformation tracking}

The biopsy workflow involving the real-time deformation tracking setup does not make use of a robot arm. As described in Chapter 14 the needle insertion error was evaluated to be $4.5 \mathrm{~mm}$ on average when targeting a specific breast phantom. In this experiment the needle was inserted by hand while the computer vision system gave feedback on the required needle orientation and remaining insertion depth. This workflow is less complex than the Sunram and MURAB workflows as no robotic system is employed, but it is also limited in the sense that movements of tissue within the breast itself cannot be tracked directly. 


\subsection{Procedure time}

The total procedure time of any workflow depends on many aspects. For example the complete MURAB workflow consists of a large number of components of which some are not fully integrated yet and cannot be directly evaluated, such as 3-D ultrasound reconstruction. The use of certain steps depends on the specific workflow chosen: elastography acquisition and deformation compensation are optional components which enhance accuracy but are not strictly necessary to perform the biopsy, while the method of real-time deformation tracking and compensation uses an entirely different workflow. Several aspects in the user interface can be optimized and streamlined to minimize the procedure time. Taking the most recent biopsy tests as a reference, the total practical procedure time of the standard MURAB workflow is multiple hours with successive biopsies on the same phantom taking about twenty minutes each.

The Stormram/Sunram workflow is more straightforward than the MURAB workflow as it primarily focuses on needle insertion only. The patient goes inside the scanner with the robot, the lesion is identified and the robotic system can acquire the sample within minutes. The full clinical workflow will involve additional steps such as setting up the robotic system, contrast injection, preoperative MRI scanning of the patient, insertion location preparation with anaesthetics, needle placement etcetera. The total duration of a fully optimized workflow is estimated to be around 30 minutes.

Like the MURAB workflow, the workflow involving biopsy under real-time deformation tracking is split into two parts: the MRI scan and the actual intervention. The interventional part can be made relatively quick, in the order of 15 minutes or less if the system is able to automatically detect and register the markers when the patient is in position. Knowing the estimated location of the lesion, it is then straightforward to provide the radiologist with the necessary information to guide the needle towards the correct location.

\subsection{Conclusion}

Three different technological systems have been developed that can guide a needle towards a target lesion only visible on MRI, in the breast. The Storm$\mathrm{ram} /$ Sunram uses a robotic system inside the MRI scanner allowing direct visualization of the target lesion, the MURAB setup is located outside the MRI scanner allowing indirect visualization of the target lesion by MRI-US fusion, and the real-time deformation tracking setup uses geometric interpolation of the elastically deformed, registered MRI scan to estimate the current location of the target lesion inside the breast.

All three technologies have its own characteristics, strengths and drawbacks. The overall targeting accuracy of the presented prototypes is in the order of 
$5 \mathrm{~mm}$. This is comparable with the golden standard, the manual MRI-guided breast biopsy procedure. Moreover, each prototype is a proof-of-concept with strong potential to improve accuracy further. There is a good chance that at least one of the presented technologies eventually allows to reliably target small-sized ( $5 \mathrm{~mm}$ diameter and smaller) lesions in human breast tissue, in a single biopsy with minimal tissue damage.

\subsubsection{Outlook}

The clinical applications of the presented technologies are not limited to breast biopsy only. Several research groups worldwide have expressed interest in the demonstrated pneumatic stepper motor technology to integrate it in their MR safe robotic systems designed for different medical interventions. Examples are in liver biopsies and ablation, actuation of endoscope systems and steering catheters through blood vessels. One recent example is a robotic system for MRI-guided endovascular interventions, developed by Abdelaziz et al. [5].

In order to boost further developments, the 3-D files of a large number of stepper motor designs have been made available to the worldwide community [44]. On the business side a company was established (Machnet Medical Robotics) which aims to develop a commercial market product based on the Sunram 5 and its patented technology.

The MURAB and real-time deformation tracking systems incorporate various technologies aimed at tracking MRI-visible lesions outside the MRI scanner. While the breast is the primary application due to its relatively high deformability, certain other organs are also subject to tissue deformations. One example is the liver which experiences periodic displacements due to breathing, and compensating these motions by using appropriate technologies could improve the accuracy of interventional procedures in this organ. Other areas of interest are the prostate and neurosurgical applications.

Like any medical technology, it will take considerable time before this scientific research eventually turns into a product for clinical use. Breast cancer is a serious disease and many women would benefit from technological advances in screening and diagnosis. In that respect the technology developed in this research may not provide an immediate improvement, but it might at least be a step on the path forward. 


\section{BIBLIOGRAPHY}

[1] M. Abayazid, J. op den Buijs, C. L. de Korte, and S. Misra. Effect of skin thickness on target motion during needle insertion into soft-tissue phantoms. In 2012 4th IEEE RAS \& EMBS International Conference on Biomedical Robotics and Biomechatronics (BioRob), pages 755-760. IEEE, jun 2012. doi: 10.1109/BioRob.2012.6290841.

[2] M. Abayazid, P. Moreira, N. Shahriari, S. Patil, R. Alterovitz, and S. Misra. Ultrasound-guided three-dimensional needle steering in biological tissue with curved surfaces. Medical Engineering 83 Physics, 37 (1):145-150, jan 2015. doi: 10.1016/j.medengphy.2014.10.005.

[3] M. Abdelaziz. Designing, implementing and integrating a controller for the MRI-compatible robotic breast biopsy system M. ( Mohamed) Essam Mohamed Kassem Abdelaziz MSc Report. PhD thesis, University of Twente, 2016.

[4] M. E. Abdelaziz, V. Groenhuis, J. Veltman, F. Siepel, and S. Stramigioli. Controlling the Stormram 2: An MRI-compatible robotic system for breast biopsy. In Proceedings - IEEE International Conference on Robotics and Automation, pages 1746-1753, 2017. doi: 10.1109/ICRA. 2017.7989206 .

[5] M. E. M. K. Abdelaziz, D. Kundrat, M. Pupillo, G. Dagnino, T. M. Y. Kwok, W. Chi, V. Groenhuis, C. Riga, S. Stramigioli, and G.-Z. Yang. Toward a Versatile Robotic Platform for Fluoroscopy and MRI-Guided Endovascular Interventions : A Pre-Clinical Study. In IEEE/RSJ International Conference on Intelligent Robots and Systems, Macau, 2019. IEEE.

[6] M. Altomonte, D. Zerbato, D. Botturi, and P. Fiorini. Simulation of deformable environment with haptic feedback on GPU. 2008 IEEE/RSJ International Conference on Intelligent Robots and Systems, IROS, pages 3959-3964, 2008. doi: 10.1109/IROS.2008.4650837. 
[7] A. O. Andrade, A. A. Pereira, S. Walter, R. Almeida, R. Loureiro, D. Compagna, and P. J. Kyberd. Bridging the gap between robotic technology and health care. Biomedical Signal Processing and Control, 10(1):65-78, mar 2014. doi: 10.1016/j.bspc.2013.12.009.

[8] D. P. Atchley, C. T. Albarracin, A. Lopez, V. Valero, C. I. Amos, A. M. Gonzalez-Angulo, G. N. Hortobagyi, and B. K. Arun. Clinical and pathologic characteristics of patients with BRCA-positive and BRCA-negative breast cancer. Journal of Clinical Oncology, 26(26):4282-4288, 2008. doi: 10.1200/JCO.2008.16.6231.

[9] F. S. Azar, D. N. Metaxas, and M. D. Schnall. A deformable finite element model of the breast for predicting mechanical deformations under external perturbations. Academic Radiology, 8(10):965-975, 2001. doi: 10.1016/S1076-6332(03)80640-2.

[10] T. P. Babarenda Gamage, P. M. Nielsen, and M. P. Nash. Clinical Applications of Breast Biomechanics. Elsevier Inc., 2017. doi: 10.1016/B978-0-12-804009-6.00010-9.

[11] J. Barber. Thick-walled Cylinders and Disks. In Solid Mechanics and Its Applications, volume 175, pages 449-486. Springer, Dordrecht, 2nd edition, 2011. doi: 10.1007/978-94-007-0295-0_10.

[12] C. Behrenbruch, K. Marias, P. Armitage, N. Moore, J. Clarke, M. Brady, J. R. Hospital, and H. Way. Prone-Supine Breast MRI Registration for Surgical Visualisation Oxford Magnetic Resonance Imaging (OMRI). Medical image understanding and analysis, pages 4-7, 2001.

[13] J. Bercoff, M. Tanter, and M. Fink. Supersonic Shear Imaging : A New Technique. IEEE Transactions on Ultrasonics, Ferroelectrics and Frequency Control, 51(4):396-409, 2004. doi: 10.1109/TUFFC.2004. 1295425.

[14] P. J. Besl and N. D. McKay. A Method for Registration of 3-D Shapes. IEEE Transactions on Pattern Analysis and Machine Intelligence, 14(2): 239-256, 1992. doi: 10.1109/34.121791.

[15] J. G. Bomers, D. G. Bosboom, G. H. Tigelaar, J. Sabisch, J. J. Fütterer, and D. Yakar. Feasibility of a 2nd generation MR-compatible manipulator for transrectal prostate biopsy guidance. European Radiology, 27(4):17761782, 2017. doi: 10.1007/s00330-016-4504-2.

[16] D. G. H. Bosboom, J. Fütterer, and J. Bosboom. Motor System, Motor, And Robot Arm Device Comprising The Same, 2017. 
[17] T. Botterill, T. Lotz, A. Kashif, and J. G. Chase. Reconstructing 3-D skin surface motion for the DIET breast cancer screening system. IEEE Transactions on Medical Imaging, 33(5):1109-1118, 2014. doi: 10.1109/ TMI.2014.2304959.

[18] L. J. Brattain, C. Floryan, O. P. Hauser, M. Nguyen, R. J. Yong, S. B. Kesner, S. B. Corn, and C. J. Walsh. Simple and effective ultrasound needle guidance system. In 2011 Annual International Conference of the IEEE Engineering in Medicine and Biology Society, pages 8090-8093. IEEE, aug 2011. doi: 10.1109/IEMBS.2011.6091995.

[19] F. Bray, J. Ferlay, I. Soerjomataram, R. L. Siegel, L. A. Torre, and A. Jemal. Global cancer statistics 2018: GLOBOCAN estimates of incidence and mortality worldwide for 36 cancers in 185 countries. CA: a cancer journal for clinicians, 68(6):394-424, 2018. doi: 10.3322/caac.21492.

[20] F. Bray, J. Ferlay, I. Soerjomataram, R. L. Siegel, L. A. Torre, and A. Jemal. Global cancer statistics 2018: GLOBOCAN estimates of incidence and mortality worldwide for 36 cancers in 185 countries. CA: a cancer journal for clinicians, 68(6):394-424, 2018. doi: 10.3322/caac.21492.

[21] T. Carter, C. Tanner, N. Beechey-Newman, D. Barratt, and D. Hawkes. MR Navigated Breast Surgery: Method and Initial Clinical Experience BT - Medical Image Computing and Computer-Assisted Intervention MICCAI 2008. In Medical Image Computing and Computer-Assisted Intervention - MICCAI, pages 356-363, 2008.

[22] M. Catalano, G. Grioli, M. Garabini, Felipe Weilemann Belo, A. Di Basco, N. Tsagarakis, and A. Bicchi. A Variable Damping module for Variable Impedance Actuation. Proceedings - IEEE International Conference on Robotics and Automation, pages 2666-2672, 2012. doi: 10.1109/ICRA.2012.6224938.

[23] Y.-h. H. Chang, Y.-t. T. Chen, C.-w. W. Chang, and C.-l. L. Lin. Development scheme of haptic-based system for interactive deformable simulation. CAD Computer Aided Design, 42(5):414-424, 2010. doi: 10.1016/j.cad.2008.10.011.

[24] D. Chapuis, R. Gassert, G. Ganesh, E. Burdet, and H. Bleuler. Investigation of a cable transmission for the actuation of MR compatible haptic interfaces. Proceedings of the First IEEE/RAS-EMBS International Conference on Biomedical Robotics and Biomechatronics, 2006, BioRob 2006, 2006:426-431, 2006. doi: 10.1109/BIOROB.2006.1639125. 
[25] T. E. Chemaly, F. J. Siepel, S. Rihana, V. Groenhuis, F. Van der Heijden, and S. Stramigioli. MRI and Stereo Vision Surface Reconstruction and Fusion. In International Conference on Advances in Biomedical Engineering, ICABME, volume 2017-Octob, pages 1-4, 2017. doi: 10.1109/ICABME.2017.8167571.

[26] Y. Chen, K. W. Kwok, and Z. T. H. Tse. An MR-Conditional HighTorque Pneumatic Stepper Motor for MRI-Guided and Robot-Assisted Intervention. Annals of Biomedical Engineering, 42(9):1823-1833, 2014. doi: $10.1007 / \mathrm{s} 10439-014-1049-\mathrm{x}$.

[27] Y. Chen, C. D. Mershon, and Z. T. H. Tse. A 10-mm MR-conditional unidirectional pneumatic stepper motor. IEEE/ASME Transactions on Mechatronics, 20(2):782-788, 2015. doi: 10.1109/TMECH.2014.2305839.

[28] Y. Chen, I. Godage, H. Su, A. Song, and H. Yu. Stereotactic Systems for MRI-Guided Neurosurgeries: A State-of-the-Art Review. Annals of Biomedical Engineering, 47(2):335-353, 2019. doi: 10.1007/ s10439-018-02158-0.

[29] M. C. Chevrier, J. David, M. E. Khoury, L. Lalonde, M. Labelle, and I. Trop. Breast Biopsies Under Magnetic Resonance Imaging Guidance: Challenges of an Essential but Imperfect Technique. Current Problems in Diagnostic Radiology, 45(3):193-204, 2016. doi: 10.1067/j.cpradiol.2015. 07.002 .

[30] D. B. Comber, J. E. Slightam, E. J. Barth, V. R. Gervasi, and R. J. Webster. Design and Precision Control of an MR-Compatible Flexible Fluidic Actuator. In Proceedings of the ASME/BATH 2013 Symposium on Fluid Power $\&$ Motion Control, pages 1-9, 2013. doi: 10.1115/fpmc2013-4481.

[31] R. H. Conley, I. M. Meszoely, J. A. Weis, T. S. Pheiffer, L. R. Arlinghaus, T. E. Yankeelov, and M. I. Miga. Realization of a biomechanical modelassisted image guidance system for breast cancer surgery using supine MRI. International Journal of Computer Assisted Radiology and Surgery, 10(12):1985-1996, 2015. doi: 10.1007/s11548-015-1235-9.

[32] J. A. Cunha, I.-c. Hsu, J. Pouliot, M. R. Iii, K. Shinohara, J. Kurhanewicz, G. Reed, and D. A. N. Stoianovici. Toward adaptive stereotactic robotic brachytherapy for prostate cancer : Demonstration of an adaptive work $\mathrm{fl}$ ow incorporating inverse planning and an MR stealth robot. Minimally Invasive Therapy, 19(4):189-202, 2010. doi: 10.3109/13645706.2010.497000.

[33] H. Delingette, S. Cotin, N. Ayache, and P. Epidaure. A hybrid elastic model allowing real-time cutting, deformations and force-feedback for 
surgery training and simulation. Proceedings Computer Animation 1999, pages 70-81, 2003. doi: 10.1109/ca.1999.781200.

[34] C. Duriez, C. Andriot, and A. Kheddar. Signorini's contact model for deformable objects in haptic simulations. 2004 IEEE/RSJ International Conference on Intelligent Robots and Systems (IROS), 4:3232-3237, 2004. doi: 10.1109/iros.2004.1389915.

[35] B. Eiben, L. Han, J. Hipwell, T. Mertzanidou, S. Kabus, T. Buelow, C. Lorenz, G. M. Newstead, H. Abe, M. Keshtgar, S. Ourselin, and D. J. Hawkes. Biomechanically guided prone-to-supine image registration of breast MRI using an estimated reference state. In Proceedings - International Symposium on Biomedical Imaging, pages 214-217. IEEE, 2013. doi: 10.1109/ISBI.2013.6556450.

[36] B. Eiben, V. Vavourakis, J. H. Hipwell, S. Kabus, C. Lorenz, T. Buelow, and D. J. Hawkes. Breast deformation modelling: comparison of methods to obtain a patient specific unloaded configuration. In Medical Imaging 2014: Image-Guided Procedures, Robotic Interventions, and Modeling, volume 9036, page 903615, 2014. doi: 10.1117/12.2043607.

[37] H. Elhawary, A. Zivanovic, Z. T. H. Tse, M. Rea, B. L. Davies, I. Young, G. Bydder, M. Payley, and M. U. Lamperth. A magnetic-resonancecompatible limb-positioning device to facilitate magic angle experiments in vivo. Proceedings of the Institution of Mechanical Engineers. Part H, Journal of engineering in medicine, 222:751-760, 2008. doi: 10.1243/ 09544119JEIM361.

[38] F. S. Farimani and S. Misra. Introducing PneuAct: ParametricallyDesigned MRI-Compatible Pneumatic Stepper Actuator. In Proceedings IEEE International Conference on Robotics and Automation, pages 200205. IEEE, 2018. doi: 10.1109/ICRA.2018.8462697.

[39] F. Faure, C. Duriez, H. Delingette, J. Allard, B. Gilles, S. Marchesseau, H. Talbot, H. Courtecuisse, G. Bousquet, I. Peterlík, and S. Cotin. SOFA; a Multi-Model Framework for Interactive Physical Simulation, volume 11. Springer, 2012. doi: 10.1007/8415_2012_125.

[40] O. Felfoul, A. Becker, C. Bergeles, and P. E. Dupont. Achieving Commutation Control of an MRI-Powered Robot Actuator. IEEE Transactions on Robotics, 31(2):387-399, 2015. doi: 10.1109/TRO.2015.2407795.

[41] E. Franco, D. Brujic, M. Rea, W. M. Gedroyc, and M. Ristic. Needleguiding robot for laser ablation of liver tumors under MRI guidance. IEEE/ASME Transactions on Mechatronics, 21(2):931-944, 2016. doi: 10.1109/TMECH.2015.2476556. 
[42] R. Gassert, R. Moser, E. Burdet, and H. Bleuler. MRI/fMRI-compatible robotic system with force feedback for interaction with human motion. IEEE/ASME Transactions on Mechatronics, 11(2):216-224, 2006. doi: 10.1109/TMECH.2006.871897.

[43] M. Greminger and B. Nelson. Deformable object tracking using the boundary element method. In 2003 IEEE Computer Society Conference on Computer Vision and Pattern Recognition (CVPR'03), volume 1, 2003. doi: 10.1109/cvpr.2003.1211366.

[44] V. Groenhuis. Specifications of pneumatic stepper motors and rotary engines, 2019. URL http://www.vincentgroenhuis.nl/wp/home/2019/ 10/11/stepper-motor-overview/.

[45] V. Groenhuis. Supplementary files for printing pneumatic devices., 2019. URL https://doi.org/10.4121/uuid: 9435e52e-0d9e-4bcc-90aa-fc1a1901622c.

[46] V. Groenhuis and S. Stramigioli. Laser-cutting pneumatics. IEEE/ASME Transactions on Mechatronics, 21(3):1604-1611, 2016. doi: 10.1109/ TMECH.2015.2508100.

[47] V. Groenhuis and S. Stramigioli. Rapid Prototyping High-Performance MR Safe Pneumatic Stepper Motors. IEEE/ASME Transactions on Mechatronics, 23(4):1843-1853, 2018. doi: 10.1109/TMECH.2018. 2840682 .

[48] V. Groenhuis, M. Chandrapal, S. Stramigioli, and X. Chen. Controlling pneumatic artificial muscles in exoskeletons with surface electromyography. 14th Mechatronics Forum International Conference, MECHATRONICS 2014, pages 451-457, 2014.

[49] V. Groenhuis, J. Veltman, and S. Stramigioli. Stormram 2: A MRICompatible Robotic System for Breast Biopsy. In Hamlyn Symposium on Medical Robotics, pages 52-53, 2016.

[50] V. Groenhuis, F. J. Siepel, J. Veltman, and S. Stramigioli. Design and characterization of Stormram 4: an MRI-compatible robotic system for breast biopsy. In IEEE/RSJ International Conference on Intelligent Robots and Systems (IROS) September 24-28, 2017, Vancouver, BC, Canada, volume 2017-Septe, pages 1746-1753, 2017. doi: 10.1109/IROS.2017.8202256.

[51] V. Groenhuis, J. Veltman, F. Siepel, and S. Stramigioli. Stormram 3: A Magnetic Resonance Imaging-Compatible Robotic System for Breast Biopsy. IEEE Robotics and Automation Magazine, 17(May):34-41, 2017. doi: 10.1109/MRA.2017.2680541. 
[52] V. Groenhuis, F. Siepel, and S. Stramigioli. Dual-Speed MR Safe Pneumatic Stepper Motors. In Proceedings of Robotics: Science and Systems, Pittsburgh, Pennsylvania, 2018. doi: 10.15607/rss.2018.xiv.030.

[53] V. Groenhuis, F. J. Siepel, and S. Stramigioli. Pneumatic Stepper Motor And Device Comprising At Least One Such Pneumatic Stepper Motor, 2018.

[54] V. Groenhuis, F. J. Siepel, M. K. Welleweerd, J. Veltman, and S. Stramigioli. Sunram 5: An MR Safe Robotic System for Breast Biopsy. In Hamlyn Symposium on Medical Robotics, pages 82-83, 2018.

[55] V. Groenhuis, F. J. F. Siepel, J. Veltman, J. K. J. van Zandwijk, and S. Stramigioli. Stormram 4: An MR Safe Robotic System for Breast Biopsy. Annals of Biomedical Engineering, 46(10):1686-1696, 2018. doi: 10.1007/s10439-018-2051-5.

[56] V. Groenhuis, F. Visentin, F. J. Siepel, B. M. Maris, D. Dall'alba, P. Fiorini, and S. Stramigioli. Analytical derivation of elasticity in breast phantoms for deformation tracking. International Journal of Computer Assisted Radiology and Surgery, 13(10):1641-1650, 2018.

[57] V. Groenhuis, F. J. Siepel, and S. Stramigioli. Miniaturization of MR Safe Pneumatic Rotational Stepper Motors. In IEEE/RSJ International Conference on Intelligent Robots and Systems, Macau, 2019. IEEE.

[58] V. Groenhuis, J. Siepel, and S. Stramigioli. Sunram 5 : An MR safe robotic system for breast biopsy, driven by pneumatic stepper motors. In M. H. Abedin-Nasab, editor, Handbook of Robotic and Image-Guided Surgery, chapter 22, pages 375-396. Elsevier, 2019.

[59] S. Groothuis, R. Carloni, and S. Stramigioli. A Novel Variable Stiffness Mechanism Capable of an Infinite Stiffness Range and Unlimited Decoupled Output Motion. Actuators, 3(2):107-123, jun 2014. doi: $10.3390 /$ act3020107.

[60] Z. Guo, T. T. L. Lun, Y. Chen, H. Su, D. T. M. Chan, K. Kwok, T. Chinese, H. Kong, and H. Kong. Novel Design of an MR-safe Pneumatic Stepper Motor for MRI-guided Robotic Interventions Division of Neurosurgery , Prince of Wales Hospital ,. In 9th Hamlyn Symposium on Medical Robotics Proceedings 2016, pages 50-51, 2016.

[61] Z. Guo, Z. Dong, K.-H. Lee, C. L. Cheung, H. C. Fu, J. D.-L. Ho, H. He, W. S. Poon, T.-M. Chan, and K.-W. Kwok. Compact Design of a Hydraulic Driving Robot for Intra-operative MRI-guided Bilateral Stereotactic Neurosurgery. IEEE Robotics and Automation Letters, 3(3):1-1, 2018. doi: 10.1109/LRA.2018.2814637. 
[62] L. Han, J. Hipwell, T. Mertzanidou, T. Carter, M. Modat, S. Ourselin, D. Hawkes, and G. Street. A hybrid fem-based method for aligning prone and supine images for image guided breast surgery. In Proceedings - International Symposium on Biomedical Imaging, pages 1239-1242. IEEE, 2011. doi: 10.1109/ISBI.2011.5872626.

[63] L. Han, J. H. Hipwell, C. Tanner, Z. Taylor, T. Mertzanidou, J. Cardoso, S. Ourselin, and D. J. Hawkes. Development of patient-specific biomechanical models for predicting large breast deformation. Physics in Medicine and Biology, 57(2):455-472, 2012. doi: 10.1088/0031-9155/57/ $2 / 455$.

[64] L. Han, J. H. Hipwell, B. Eiben, D. Barratt, M. Modat, S. Ourselin, and D. J. Hawkes. A nonlinear biomechanical model based registration method for aligning prone and supine $\mathrm{mr}$ breast images. IEEE Transactions on Medical Imaging, 33(3):682-694, 2014. doi: 10.1109/TMI.2013. 2294539 .

[65] E. a. Hauth, H. J. Jaeger, J. Lubnau, S. Maderwald, F. Otterbach, R. Kimmig, and M. Forsting. MR-guided vacuum-assisted breast biopsy with a handheld biopsy system: Clinical experience and results in postinterventional MR mammography after 24 h. European Radiology, 18:168176, 2008. doi: 10.1007/s00330-007-0704-0.

[66] G. A. Hendriks, C. Chen, H. H. Hansen, and C. L. De Korte. Quasistatic elastography and ultrasound plane-wave imaging: The effect of beam-forming strategies on the accuracy of displacement estimations. IEEE International Ultrasonics Symposium, IUS, 2017. doi: 10.1109/ ULTSYM.2017.8091559.

[67] M. Ho, Y. Kim, S. S. Cheng, R. Gullapalli, and J. P. Desai. Design, development, and evaluation of an MRI-guided SMA spring-actuated neurosurgical robot. International Journal of Robotics Research, 34(8): 1147-1163, 2015. doi: 10.1177/0278364915579069.

[68] J. Hong, T. Dohi, M. Hashizume, K. Konishi, and N. Hata. An ultrasound-driven needle-insertion robot for percutaneous cholecystostomy. Physics in Medicine and Biology, 49(3):441-455, feb 2004. doi: 10.1088/0031-9155/49/3/007.

[69] S. Y. Huang, J. M. Boone, K. Yang, N. J. Packard, S. E. McKenney, N. D. Prionas, K. K. Lindfors, and M. J. Yaffe. The characterization of breast anatomical metrics using dedicated breast CT. Medical Physics, 38(4):2180-2191, 2011. doi: 10.1118/1.3567147. 
[70] N. Hungr, I. Bricault, P. Cinquin, and C. Fouard. Design and Validation of a CT- and MRI-Guided Robot for Percutaneous Needle Procedures. IEEE Transactions on Robotics, 32(4):973-987, 2016. doi: 10.1109/TRO. 2016.2588884 .

[71] D. L. James and D. K. Pai. A unified treatment of elastostatic contact simulation for real time haptics. ACM SIGGRAPH 2005 Courses, 2(1): 141, 2005. doi: 10.1145/1198555.1198614.

[72] M. Jäntsch. Anthrob - A Printed Anthropomimetic Robot. In 13th IEEERAS International Conference on Humanoid Robots (Humanoids)., pages 342-347, 2013.

[73] Joline E. Brandenburg, S. F. Eby, P. Song, H. Zhao, J. S. Brault, S. Chen, and K.-N. An. Ultrasound Elastography: The New Frontier in Direct Measurement of Muscle Stiffness. Archives of Physical Medicine and Rehabilitation, 95(11):2207 - 2219, 2014. doi: https://doi.org/10.1016/j. apmr.2014.07.007.

[74] M. Katna. Thick walled cylinders, 2019. URL http://www.engr .mun. ca/\{ $\}$ katna/5931/ThickWalledCylinders (corrected).pdf.

[75] M. Kaya, E. Senel, A. Ahmad, O. Orhan, and O. Bebek. Real-time needle tip localization in $2 \mathrm{D}$ ultrasound images for robotic biopsies. In 2015 International Conference on Advanced Robotics (ICAR), pages 4752. IEEE, jul 2015. doi: 10.1109/ICAR.2015.7251432.

[76] J. Kettenbach, G. Kronreif, M. Figl, M. Fürst, W. Birkfellner, R. Hanel, and H. Bergmann. Robot-assisted biopsy using ultrasound guidance: initial results from in vitro tests. European Radiology, 15(4):765-771, apr 2005. doi: 10.1007/s00330-004-2487-x.

[77] R. Kojcev, B. Fuerst, O. Zettinig, J. Fotouhi, S. C. Lee, B. Frisch, R. Taylor, E. Sinibaldi, and N. Navab. Dual-robot ultrasound-guided needle placement: closing the planning-imaging-action loop. International Journal of Computer Assisted Radiology and Surgery, 11(6):1173-1181, jun 2016. doi: 10.1007/s11548-016-1408-1.

[78] R. Kokes, K. Lister, and R. Gullapalli. Towards a needle driver robot for radiofrequency ablation of tumors under continuous MRI. ... , 2008. Icra 2008. . ., 1(Md):2509-2514, 2008.

[79] T. Laliberté, C. M. Gosselin, and G. Côté. A rapid prototyping framework for fast and cost-effective design of robotic mechanism prototypes. IEEE Robotics and Automation Magazine, 8(3):43-52, 2001. 
[80] C. H. Lee, D. D. Dershaw, D. Kopans, P. Evans, B. Monsees, D. Monticciolo, R. J. Brenner, L. Bassett, W. Berg, S. Feig, E. Hendrick, E. Mendelson, C. D'Orsi, E. Sickles, and L. W. Burhenne. Breast Cancer Screening With Imaging: Recommendations From the Society of Breast Imaging and the ACR on the Use of Mammography, Breast MRI, Breast Ultrasound, and Other Technologies for the Detection of Clinically Occult Breast Cancer. Journal of the American College of Radiology, 7(1):18-27, 2010. doi: 10.1016/j.jacr.2009.09.022.

[81] C. D. Lehman, E. R. DePeri, S. Peacock, M. D. McDonough, W. B. DeMartini, and J. Shook. Clinical Experience with MRI-Gieded VacuumAssisted Breast Biopsy. AJR. American journal of roentgenology, 184(6): 1782-1787, 2005.

[82] M. Li, D. Mazilu, A. Kapoor, and K. a. Horvath. MRI Compatible Robot Systems for Medical Intervention. In Advances in Robot Manipulators, pages 443-459. IntechOpen, 2010. doi: 10.5772/9675.

[83] K. Liang, A. J. Rogers, E. D. Light, D. von Allmen, and S. W. Smith. Three-Dimensional Ultrasound Guidance of Autonomous Robotic Breast Biopsy: Feasibility Study. Ultrasound in Medicine 8 Biology, 36(1):173177, jan 2010. doi: 10.1016/j.ultrasmedbio.2009.08.014.

[84] K. Liang, A. J. Rogers, E. D. Light, D. von Allmen, and S. W. Smith. Simulation of Autonomous Robotic Multiple-Core Biopsy by 3D Ultrasound Guidance. Ultrasonic Imaging, 32(2):118-127, apr 2010. doi: $10.1177 / 016173461003200205$.

[85] G.-R. Liu and S. S. Quek. The finite element method: a practical course. Butterworth-Heinemann, 2013.

[86] R. R. Ma, L. U. Odhner, and A. M. Dollar. A modular, open-source 3D printed underactuated hand. Proceedings - IEEE International Conference on Robotics and Automation, pages 2737-2743, 2013. doi: 10.1109/ICRA.2013.6630954.

[87] A. Maciel, R. Boulic, and D. Thalmann. Deformable Tissue Parameterized by Properties of Real Biological Tissue. In International Symposium on Surgery Simulation and Soft Tissue Modeling, volume 2673, pages 74-87, 2003. doi: 10.1007/3-540-45015-7_8.

[88] M. Z. Mahmoud, M. Aslam, M. Alsaadi, M. A. Fagiri, and B. Alonazi. Evolution of Robot-assisted ultrasound-guided breast biopsy systems. Journal of Radiation Research and Applied Sciences, 11(1):89-97, jan 2018. doi: 10.1016/j.jrras.2017.11.005. 
[89] V. Mallapragada, N. Sarkar, and T. Podder. Robot-Assisted Real-Time Tumor Manipulation for Breast Biopsy. IEEE Transactions on Robotics, 25(2):316-324, apr 2009. doi: 10.1109/TRO.2008.2011418.

[90] V. G. Mallapragada, N. Sarkar, and T. K. Podder. Robotic system for tumor manipulation and ultrasound image guidance during breast biopsy. In 2008 30th Annual International Conference of the IEEE Engineering in Medicine and Biology Society, pages 5589-5592. IEEE, aug 2008. doi: 10.1109/IEMBS.2008.4650481.

[91] G. Megali, O. Tonet, C. Stefanini, M. Boccadoro, V. Papaspyropoulos, L. Angelini, and P. Dario. A Computer-Assisted Robotic UltrasoundGuided Biopsy System for Video-Assisted Surgery. In Lecture Notes in Computer Science (including subseries Lecture Notes in Artificial Intelligence and Lecture Notes in Bioinformatics), volume 2208, pages 343-350. Springer, 2001. doi: 10.1007/3-540-45468-3_41.

[92] R. Monfaredi, K. Cleary, and K. Sharma. MRI Robots for Needle-Based Interventions: Systems and Technology. Annals of Biomedical Engineering, 46(10):1479-1497, 2018. doi: 10.1007/s10439-018-2075-x.

[93] P. Moreira and S. Misra. MR-Compatible Robot for Needle-Based Prostate Interventions. In Proc. Hamlyn Symp. Medical Robotics, pages 7-8, 2016.

[94] R. M. Murray, Z. Li, and S. S. Sastry. A Mathematical Introduction to Robotic Manipulation. CRC Press, dec 1994. doi: 10.1201/ 9781315136370 .

[95] T. R. Nelson, A. Tran, H. Fakourfar, and J. Nebeker. Positional Calibration of an Ultrasound Image-Guided Robotic Breast Biopsy System. Journal of Ultrasound in Medicine, 31(3):351-359, mar 2012. doi: 10.7863/jum.2012.31.3.351.

[96] K. Nightingale. Acoustic Radiation Force Impulse (ARFI) Imaging: A Review. Current Medical Imaging Reviews, 7(4):328-339, 2011. doi: 10. $2174 / 157340511798038657$.

[97] A. V. Nikolaev, H. H. G. Hansen, L. de Jong, R. Mann, F. Siepel, A. Nikolaev, E. Tagliabue, B. Maris, V. Groenhuis, M. Caballo, I. Sechopoulos, and C. L. de Korte. Ultrasound-guided breast biopsy of ultrasound occult lesions using multimodality image co-registration and tissue displacement tracking. In International Congress on Ultrasonics 2019, page 45, Bruges, Belgium, 2019. doi: 10.1117/12.2513630. 
[98] F. P. Oliveira and J. M. R. Tavares. Medical image registration: A review, 2014. URL http://dx.doi.org/10.1080/10255842.2012.670855.

[99] A. Y. Park and B. K. Seo. Real-Time MRI Navigated Ultrasound for Preoperative Tumor Evaluation in Breast Cancer Patients: Technique and Clinical Implementation. Korean Journal of Radiology, 17(5):695, 2016. doi: 10.3348/kjr.2016.17.5.695.

[100] D. M. Parkin, P. Pisani, and J. Ferlay. Global cancer statistics. CA Cancer J Clin, 49(2):1,33-64, 1999. doi: 10.3322/caac.20107.Available.

[101] P. Pathmanathan, D. J. Gavaghan, J. P. Whiteley, S. J. Chapman, and J. M. Brady. Predicting tumor location by modeling the deformation of the breast. IEEE Transactions on Biomedical Engineering, 55(10): 2471-2480, 2008. doi: 10.1109/TBME.2008.925714.

[102] F. Pediconi, C. Catalano, A. Roselli, V. Dominelli, S. Cagioli, A. Karatasiou, A. Pronio, M. A. Kirchin, and R. Passariello. The Challenge of Imaging Dense Breast Parenchyma. Investigative Radiology, 44(7):412421, jul 2009. doi: 10.1097/RLI.0b013e3181a53654.

[103] N. H. G. M. Peters, I. H. M. B. Rinkes, N. P. A. Zuithoff, W. P. T. M. Mali, K. G. M. Moons, and P. H. M. Peeters. Meta-analysis of MR imaging in the diagnosis of breast lesions. Radiology, 2009. doi: https: //doi.org/10.1148/radiol.2461061298.

[104] G. Picinbono, H. Delingette, N. Ayache, H. Delingette, and N. Ayache. Non-linear anisotropic elasticity for real-time surgery simulation. Graphical Models, 65(5):305-321, 2003. doi: 10.1016/S1524-0703(03)00045-6.

[105] E. R. Price. Magnetic Resonance Imaging-Guided Biopsy of the Breast: Fundamentals and Finer Points, 2013.

[106] M. Rahimzadeh, A. R. Baghestani, M. R. Gohari, and M. A. Pourhoseingholi. Estimation of the Cure Rate in Iranian Breast Cancer Patients. Asian Pacific Journal of Cancer Prevention, 15(12):4839-4842, jun 2014. doi: 10.7314/APJCP.2014.15.12.4839.

[107] L. Roose, W. D. Maerteleire, W. Mollemans, W. De Maerteleire, W. Mollemans, and P. Suetens. Validation of different soft tissue simulation methods for breast augmentation. International Congress Series, 1281:485-490, 2005. doi: 10.1016/j.ics.2005.03.126.

[108] D. Rueckert, L. Sonoda, C. Hayes, D. Hill, M. Leach, and D. Hawkes. Nonrigid registration using free-form deformations: application to breast MR images. IEEE Trans. Med. Imag., 18(8):712-21, 1999. doi: 10.1109/ 42.796284 . 
[109] H. Sajima, I. Sato, H. Yamashita, T. Dohi, and K. Masamune. Twodof non-metal manipulator with pneumatic stepping actuators for needle puncturing inside open-type MRI. World Automation Congress (WAC), 2010, pages 3-8, 2010.

[110] H. Sajima, H. Kamiuchi, K. Kuwana, T. Dohi, and K. Masamune. MRsafe pneumatic rotation stepping actuator. Journal of Robotics and Mechatronics, 24(5):820-826, 2012.

[111] A. Samani, J. Bishop, M. J. Yaffe, and D. B. Plewes. Biomechanical 3-D finite element modeling of the human breast using MRI data. IEEE Transactions on medical imaging, 20(4):271-279, 2001. doi: 10.1109/42. 921476.

[112] J. A. Schnabel, C. Tanner, A. D. Castellano-Smith, A. Degenhard, M. O. Leach, D. R. Hose, D. L. G. Hill, and D. J. Hawkes. Validation of nonrigid image registration using finite-element methods: Application to breast MR images. IEEE Transactions on Medical Imaging, 22(2): 238-247, 2003. doi: 10.1109/TMI.2002.808367.

[113] R. Secoli, M. Robinson, M. Brugnoli, and F. Rodriguez y Baena. A low-cost, high-field-strength magnetic resonance imaging-compatible actuator. Proceedings of the Institution of Mechanical Engineers, Part H: Journal of Engineering in Medicine, 229(3):215-224, 2015. doi: $10.1177 / 0954411915574306$.

[114] F. G. Shellock and A. Spinazzi. MRI safety update 2008: Part 2, screening patients for MRI. American Journal of Roentgenology, 191(4):1140-1149, 2008. doi: 10.2214/AJR.08.1038.2.

[115] C. M. Sommerich, S. A. Lavender, K. Evans, E. Sanders, S. Joines, S. Lamar, R. Z. Radin Umar, W.-t. Yen, J. Li, S. Nagavarapu, and J. A. Dickerson. Collaborating with cardiac sonographers to develop work-related musculoskeletal disorder interventions. Ergonomics, 59(9): 1193-1204, sep 2016. doi: 10.1080/00140139.2015.1116613.

[116] R. Spoor, M. Abayazid, F. Siepel, V. Groenhuis, and S. Stramigioli. Design and evaluation of a robotic needle steering manipulator for imageguided biopsy. In BME 2017, volume 6, 2017.

[117] R. Spoor, M. Abayazid, F. Siepel, V. Groenhuis, and S. Stramigioli. Design and evaluation of a robotic needle steering manipulator for imageguided biopsy. In 6th Dutch Bio-Medical Engineering Conference 201\%, Egmond aan Zee, 2017. 
[118] D. Stoianovici, A. Patriciu, D. Petrisor, D. Mazilu, and L. Kavoussi. A New Type of Motor: Pneumatic Step Motor. IEEE/ASME Transactions on Mechatronics, 2007.

[119] D. Stoianovici, C. Kim, G. Srimathveeravalli, P. Sebrecht, D. Petrisor, J. Coleman, S. B. Solomon, and H. Hricak. MRI-safe robot for endorectal prostate biopsy. IEEE/ASME Transactions on Mechatronics, 19(4): 1289-1299, 2014. doi: 10.1109/TMECH.2013.2279775.

[120] D. Stoianovici, C. Kim, D. Petrisor, C. Jun, S. Lim, M. W. Ball, A. Ross, K. J. MacUra, and M. E. Allaf. MR Safe Robot, FDA Clearance, Safety and Feasibility of Prostate Biopsy Clinical Trial. IEEE/ASME Transactions on Mechatronics, 22(1):115-126, 2017. doi: 10.1109/TMECH.2016. 2618362 .

[121] D. Stoianovici, C. Jun, S. Lim, P. Li, D. Petrisor, S. Fricke, K. Sharma, and K. Cleary. Multi-imager compatible, MR safe, remote center of motion needle-guide robot. IEEE Transactions on Biomedical Engineering, 65(1):165-177, 2018. doi: 10.1109/TBME.2017.2697766.

[122] H. Su, M. Zervas, G. A. Cole, C. Furlong, and G. S. Fischer. Realtime MRI-guided needle placement robot with integrated fiber optic force sensing. Proceedings - IEEE International Conference on Robotics and Automation, pages 1583-1588, 2011. doi: 10.1109/ICRA.2011.5979539.

[123] H. Su, D. C. Cardona, W. Shang, A. Camilo, G. A. Cole, D. C. Rucker, R. J. Webster, and G. S. Fischer. A MRI-guided concentric tube continuum robot with piezoelectric actuation: A feasibility study. Proceedings - IEEE International Conference on Robotics and Automation, pages 1939-1945, 2012. doi: 10.1109/ICRA.2012.6224550.

[124] H. Su, G. a. Cole, and G. S. Fischer. High-Field MRI-Compatible Needle Placement Robots for Prostate Interventions: Pneumatic and Piezoelectric Approaches. Intelligent Systems Reference Library, 26:3-32, 2012. doi: 10.1007/978-3-642-23363-0_1.

[125] H. Su, W. Shang, G. Cole, G. Li, K. Harrington, A. Camilo, J. Tokuda, C. M. Tempany, N. Hata, and G. S. Fischer. Piezoelectrically Actuated Robotic System for MRI-Guided Prostate Percutaneous Therapy. IEEE/ASME Transactions on Mechatronics, 20(4):1920-1932, 2015. doi: 10.1109/TMECH.2014.2359413.

[126] N. Tanaiutchawoot, C. Wiratkapan, B. Treepong, and J. Suthakorn. On the design of a biopsy needle-holding robot for a novel breast biopsy 
robotic navigation system. In The 4th Annual IEEE International Conference on Cyber Technology in Automation, Control and Intelligent, pages 480-484. IEEE, jun 2014. doi: 10.1109/CYBER.2014.6917511.

[127] L. A. Torre, F. Bray, R. L. Siegel, J. Ferlay, J. Lortet-Tieulent, and A. Jemal. Global Cancer Statistics, 2012. CA: A Cancer Journal for Clinicians, 65(2):87-108, 2015. doi: 10.3322/caac.21262.

[128] J. J. A. S. Uiterkamp, V. Groenhuis, F. J. Siepel, and S. Stramglioli. Design and Implementation of Autnomous Robotic Scanning of the Breast. In 6th Dutch Bio-Medical Engineering Conference 2017, volume 30, page 7522, Egmond, 2017.

[129] J. Veltman, C. Boetes, T. Wobbes, J. G. Blickman, and J. O. Barentz. Magnetic Resonance-Guided Biopsies and Localizations of the Breast. Investigative Radiology, 40(6):379-384, 2005.

[130] F. Visentin, V. Groenhuis, B. Maris, D. Dall'Alba, F. Siepel, S. Stramigioli, and P. Fiorini. Iterative simulations to estimate the elastic properties from a series of MRI images followed by MRI-US validation. Medical and Biological Engineering and Computing, pages 913-924, 2018. doi: 10.1007/s11517-018-1931-z.

[131] G. J. Vrooijink, M. Abayazid, and S. Misra. Real-time three-dimensional flexible needle tracking using two-dimensional ultrasound. In 2013 IEEE International Conference on Robotics and Automation, pages 1688-1693. IEEE, may 2013. doi: 10.1109/ICRA.2013.6630797.

[132] B. Whelan, G. P. Liney, J. A. Dowling, R. Rai, L. Holloway, L. McGarvie, I. Feain, M. Barton, M. Berry, R. Wilkins, and P. Keall. An MRI-compatible patient rotation system - Design, construction, and first organ deformation results:. Medical Physics, 44(2):581-588, 2017. doi: 10.1002/mp.12065.

[133] J. P. Whitney, M. F. Glisson, E. L. Brockmeyer, and J. K. Hodgins. A low-friction passive fluid transmission and fluid-tendon soft actuator. In IEEE International Conference on Intelligent Robots and Systems, pages 2801-2808, 2014. doi: 10.1109/IROS.2014.6942946.

[134] Y. Xu, Q. Zhang, and G. Liu. Cutting performance orthogonal test of single plane puncture biopsy needle based on puncture force. In AIP Conference Proceedings, page 030016, 2017. doi: 10.1063/1.4981581.

[135] B. Yang, U. X. Tan, A. B. McMillan, R. Gullapalli, and J. P. Desai. Design and control of a 1-DOF MRI-compatible pneumatically actuated robot with long transmission lines. IEEE/ASME Transactions on Mechatronics, 16(6):1040-1048, 2011. doi: 10.1109/TMECH.2010.2071393. 
[136] B. Yang, S. Roys, U.-X. Tan, M. Philip, H. Richard, R. P. Gullapalli, and J. P. Desai. Design, Development, and Evaluation of a MasterSlave Surgical System for Breast Biopsy under Continuous MRI. The International Journal of Robotics Research, 33(4):616-630, 2014. doi: 10.1177/0278364913500365.

[137] H. Zonderland. BI-RADS-classificatie bij mamma-carcinoom. Nederlands Tijdschrift voor Oncologie, 6(4):145-157, 2009. 


\section{DANKWOORD}

Aan vele mensen ben ik dank verschuldigd voor inhoudelijke hulp en mentale support bij mijn promotie-onderzoek en het schrijven van dit proefschrift. Om de dikte van dit boekwerk een beetje binnen de perken te houden ga ik niet iedere persoon specifiek noemen. Wel wil ik graag de volgende uitzonderingen maken:

Stefano, het was een voorrecht om jouw promovendus te mogen zijn. Vele diepgaande discussies hebben wij gevoerd over bestaande en nieuwe onderzoeken. Jouw inspirerende woorden brachten deze tot grote hoogten, en altijd gaf je mij de ruimte om iets nieuws te proberen of dingen op mijn eigen manier te doen. Soms nam je me mee naar een goed restaurant, en zodoende ben ik ook van sushi gaan houden. Met al de 3-D printers welke je in ons lab hebt gebracht kan ik mij geen betere vakgroep voorstellen dan waar ik nu zit.

Françoise, als co-promotor speelde jij een belangrijke rol in mijn $\mathrm{PhD}$ traject. Jij hebt allereerst het MURAB team bij elkaar weten te krijgen en het wetenschappelijke onderzoek daarvan helemaal op de rails gezet. Wat eerst allemaal losse eilandjes waren in vier verschillende landen is door jouw gedrevenheid meer en meer een goed geolied team geworden. Inhoudelijk hebben wij vele goede discussies gevoerd over allerlei onderzoeksaspecten en daaruit allerlei papers geschreven. Dankjewel voor alles!

Aan mijn geweldige (oud-)kamergenoten in 'Best Office': dankjewel voor alle gezelligheid en grappige momenten. Marcel, Stefan, Frieda, Gerjan, Rob, Hengameh, Martijn, Jornt, Toon, Maaike, Olga, Haye, Mourad: mag ik jullie allen nogmaals hartelijk danken voor alle gezondheidswensen als ik weer eens moest niezen. Ook aan Ferdi, Dayna, Nicolò en andere collega's van de vakgroep: het is fijn om met jullie samen te werken aan verschillende projecten.

Marcel, samen hebben wij vele jaren aan de MURAB robot gewerkt en ook een tijd aan de Sunram 5. Zonder jouw belangrijke bijdragen zouden we nooit zover zijn gekomen als waar we nu staan. Jij laat zien hoeveel meer wij met teamwork kunnen bereiken. Hartelijk dank voor deze, en ook dat je mijn paranimf wilt zijn. 
Abe, het hele Stormram/Sunram project is mede door jouw geïnitieerd. Waar een gesprekje met een professor op een overzeese conferentie al wel niet toe kan leiden. Ik kan iedere ondernemer aanraden om ook eens een professor aan te spreken, naar jouw goede voorbeeld!

Jeroen, als radioloog speelde jij een sleutelrol in de klinische kant van het hele onderzoek. Jij liet mij de MRI-geleide biopsieprocedure zien en was altijd bereid om het een en ander nader toe te lichten. Dankjewel!

Nico, jij mag het pneumatische stappenmotor-patent gaan exploiteren in jullie spin-off. Waar ik je specifiek voor wil bedanken is de toestemming om de 3-D tekeningen van de gepatenteerde stappenmotoren vrij beschikbaar te maken. Namens de wereldwijde open-source community, hartelijk dank daarvoor!

Gerben, Sander, Henny en Marcel: als technici van onze vakgroep hebben jullie een enorme bijdrage geleverd aan de MURAB en Stormram projecten, met name door het 3-D printen en lasersnijden van haast ontelbaar veel onderdelen en het regelen van allerlei andere spullen en apparatuur. Ook hebben jullie het patiëntbed van de MURAB setup ontworpen en opgebouwd. Voor dit allen, hartelijk dank!

Jolanda, als secretaresse stond jij altijd voor mij klaar als er iets geregeld moest worden voor een project, dienstreis, presentatie of iets anders. Mensen als jij worden nooit in wetenschappeljke papers genoemd, dus bij deze wil ik jou in het bijzonder bedanken voor alles wat je voor mij hebt gedaan in de afgelopen jaren. Dankjewel!

Jordy en Frank, jullie zijn meesters van de MRI scanners. Ik kon altijd zo bij jullie binnenvallen als ik de scanner wilde gebruiken of er vragen over had. Dat wij inmiddels zelfs twee eigen MRI scanners op de UT hebben is gewoonweg awesome!

Onze gewaardeerde partners binnen het MURAB project: we kunnen trots zijn op alle resultaten die we tot dusver hebben behaald! Ik wil in het bijzonder Eleonora, Francesco, Bogdan, Anton, Leon, Juan, Johannes en Felix bedanken voor de prettige samenwerking binnen de "deformables" groep.

Aan de vele studenten die ik heb mogen begeleiden: jullie zijn geweldig! Ik kan enorm genieten van jullie enthousiasme en doorzettingsvermogen om zoveel mogelijk uit jullie afstudeerproject te halen. Het geeft telkens veel voldoening als er weer een succesvolle robot-demonstratie of presentatie uit is gekomen.

Tim de Man, veel van onze wetenschappelijke video's zijn door jou van muziek en voice-over voorzien. Jouw muzikale composities zijn ijzersterk en een lust voor het oor. Moge jouw klanken in nog vele toekomstige compilaties weerklinken!

Huisgenoten van 't Piepke: het is inspirerend om met jullie onder één dak te wonen. Ook aan de oud-huisgenoten: dank voor de heerlijke gerechten, de gezelligheid en motiverende gesprekken bij het schrijven van mijn thesis! 
Jongleurs van CatchUp: menig object hebben we naar elkaar toegesmeten en weer opgevangen, en festivals in heel Europa bezocht. En ook aan de schakers van ESG Max Euwe en andere vrienden: vele schaakpartijtjes, bordspellen, zeiltochtjes, bergwandelingen, dansfeesten en andere activiteiten hebben wij ondernomen, en ik hoop deze nog lange tijd met jullie voort te zetten!

Aan mijn ouders, zus, aangetrouwden en andere familie: dank voor alle mentale ondersteuning tijdens mijn onderzoek. Papa en mama, jullie hebben mij grootgebracht met dozen vol technisch lego waardoor mijn creativiteit helemaal tot bloei is gekomen. Zelfs de paplepel was van lego, zeg maar. Zuslief Jessica, dank dat jij mijn paranimf wilt zijn!

Christa, als levenspartner heb je mij met alle liefde ondersteund bij schrijven van dit proefschrift. Mijn laptop sleepte ik overal mee naartoe en dat vond jij allemaal best. Terwijl ik zat te typen maakte jij de lekkerste baksels. Ik hoop samen met jou een mooie toekomst in te gaan! 



\section{BIOGRAPHY}

Vincent Groenhuis was born on November 15, 1983 in Nordhorn, Germany. Six weeks after birth, his parents relocated to Leiden and four years later to Enschede. He obtained his Gymnasium diploma at Bonhoeffer College, Enschede with flying colors and also participated in various national and international olympiads in his high-school period, becoming Dutch champion in Informatics in 2000, winning two bronze medals at International Mathematical Olympiad (2000, 2001), and one at the International

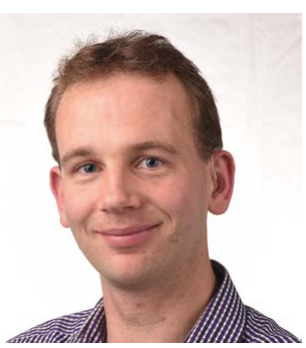
Olympiad in Informatics (2001). Based on Vincent's broad interests in engineering subjects, he obtained the propedeuses in both Computer Science and Applied Physics in the same year, continuing with Computer Science and eventually obtaining the Bachelor of Science degree on the topic of navigation of an autonomous vehicle using a singular light sensor in a known environment in 2006. During his BSc period Vincent also participated in the World Solar Challenge with the Raedthuys Solar Team in 2005, finishing ninth as the best newcomer team. It took some time before successfully starting the master's in Embedded Systems. During his MSc study Vincent was offered to do his thesis on the topic of MRI-guided breast biopsy, as proposed by prof.dr.ir. Stefano Stramigioli, dr. Jeroen Veltman and Abe van der Werf from Machnet BV. This led to the development of the Stormram 1 robot and Vincent obtained his MSc degree on this project in 2014. Vincent was then awarded a Pioneers in Healthcare voucher for further development and also the MURAB project grant was written and submitted in this period. This grant got approved and Vincent started his PhD in the MURAB project from 2016 on with development on Stormram 3, 4 and Sunram 5 continuing in the background, resulting in $15+$ peer-reviewed papers and two awards at the Hamlyn Surgical Robot Challenge. Despite being severily hearing impaired, Vincent enjoys making music with various instruments on basic level. His other hobbies are juggling, dancing, sailing, photography, 3-D design and travelling all over the world, preferably in the mountains. 


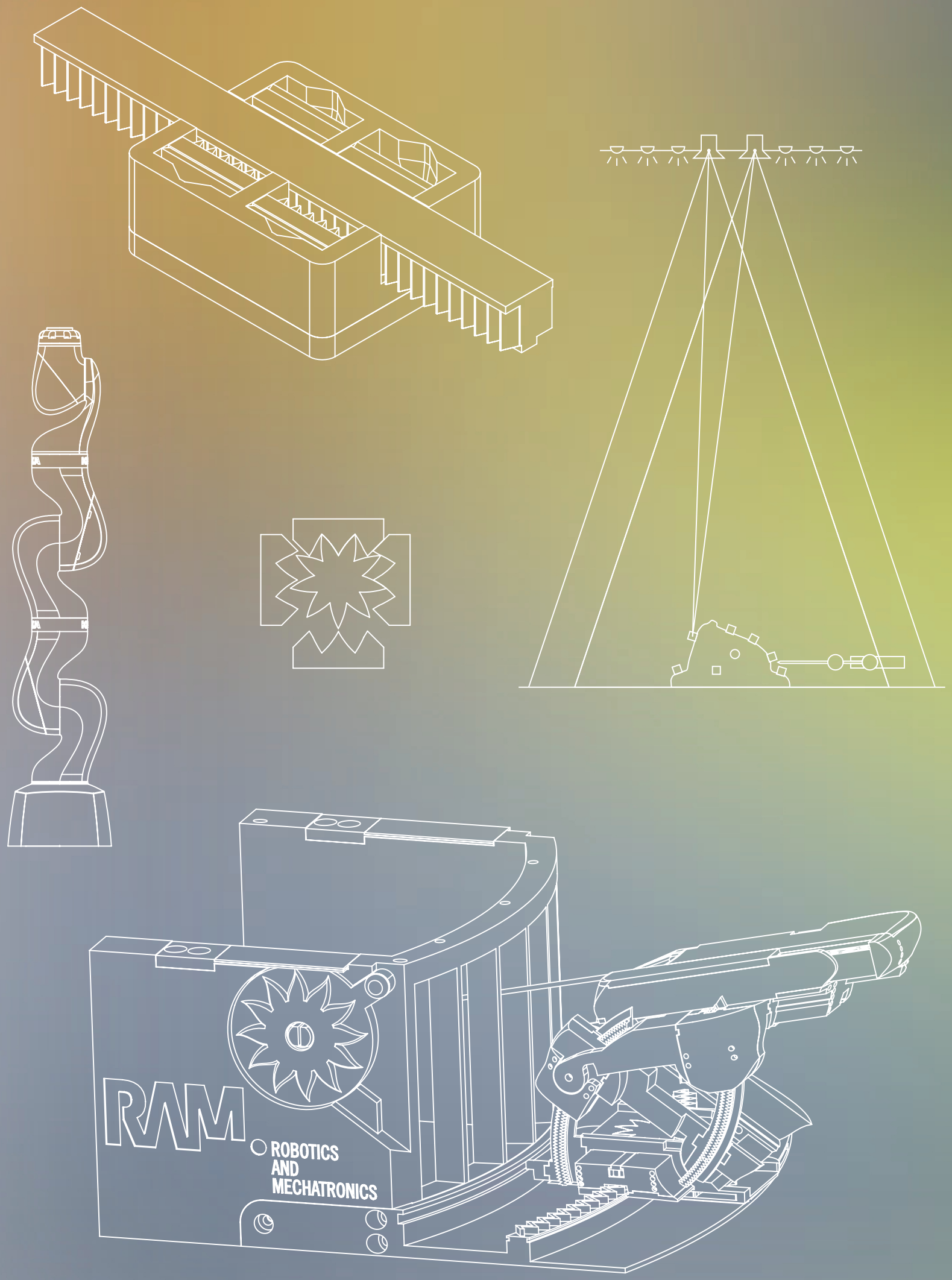

